\title{
An Optical Study of Dwarf Galaxies with Narrow HI linewidths: \\ Dark Matter and the Tully-Fisher Relation at the Faint End
}

\author{
Richard Josephı Patterson \\ Charlottesville, Virginia \\ B. A., University of Virginia, 1984 \\ M. A., Universily of Virginia, 1989
}

A Disscrtation Presented to the Graduate Faculty of the Liniversity of Virginia in Candidacy for the I)cgrec of I)octor of Philosophy

1)epartment of Astronomy

l'niversity of Virginia.

May, 1995

David C. Brydges

Robert W. O'Connell

Mercedes T. Richards

Morton S. Roberts

Lawrence E. Thomas

D. Mark Whittle

Trinlı X. Thuanı 


\section{Contents}

Abstract $\quad$ ix

1 General Introduction 1

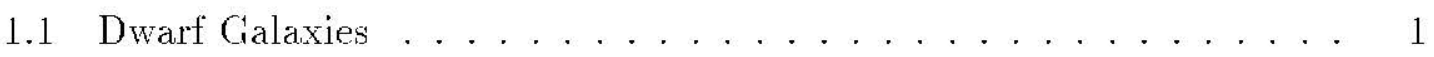

1.2 Star formation . . . . . . . . . . . . . . . . 3

1.3 Galaxy Formation ................... 6

1.4 Dark Matter . . . . . . . . . . . . . . . †

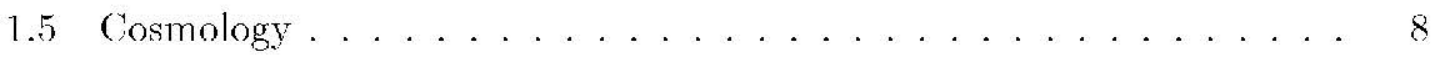

1.6 T'ully Fisher Relation .................... 8

1.7 Ontline of the Thesis .................... 12

2 CCD Surface Photometry of Dwarf Galaxies 13

2.1 Sample Solection . . . . . . . . . . . . . . 13

2.2 Observations . . . . . . . . . . . . . . . 17

2.3 Dala Reduction . . . . . . . . . . . . . . . 23

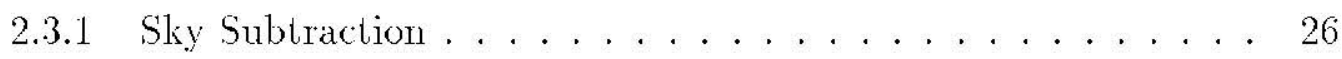

2.4 Photometric Calibration . . . . . . . . . . . . . 27

2.4.1 Flux Calibration of Galaxy Images . . . . . . . . . . 32

2.5 Ellipse Fitting and Radial Profile Extraction . . . . . . . . 33 
2.6 Comparison with Previous Data . . . . . . . . . . 36

2.7 Presentation of the Data ...................... 39

3 Corrections to Magnitude and HI Linewidth $\quad 122$

3.1 Structural Parameters . . . . . . . . . . . . . . 122

3.1.1 Extinction and Orientation Corrections to Surface Brightness 124

3.1.2 Exponential I)isk Profiles ............... 126

3.2 Adopted loistances . . . . . . . . . . . . . . . . 132

3.3 HI linewidth Corrections .................. 135

4 Tully-Fisher Relation for Dwarfs with Narrow HI linewidths 137

1.1 T'ully Fisher Relation for Normal Galaxics . . . . . . . . . . . 137

1.1.1 Historical Background and 'l'heoretical Basis . . . . . . . . 137

4.1.2 Nonlinearity of the Tully-Fisher relation . . . . . . . . . . . . 141

4.2 Motivation for this Work . . . . . . . . . . . . . . . . . . . 142

4.3 Tully-Fisher Relation for the Sample . . . . . . . . . . . . . . . 143

4.3 .1 The Basic Relation . . . . . . . . . . . . . 143

4.3.2 Correlations with dispersion ................ 146

4.4 Modified Version of the Line Width . . . . . . . . . . . 150

4.5 Turbulence Alone? . . . . . . . . . . . . . . . . 152

1.6 Is the Fffect Real? . . . . . . . . . . . . . 156

1.6.1 The relation for the $/$-band . . . . . . . . . . . . 161

1.7 Surface Brightness and Color: I)istance Independent 'lests . . . . . . 165

4.8 Evidence for Mass-Loss within a Dominant Dark IIalo . . . . . . . . 168

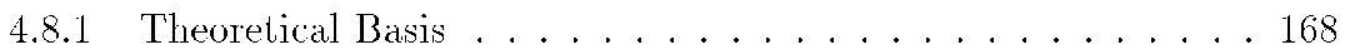

4.8 .2 Observational Evidence . . . . . . . . . . . . . . 171 
4.9 Mass to Light Dependence in Massive Galaxies: Implications for Spirals from the Tully-Fisher Relation . . . . . . . . . . . . 173

4.10 A Distance Indicator for Dwarfs? . . . . . . . . . . . . . 174

5 Global Properties and Comparative Structure of Dwarfs 177

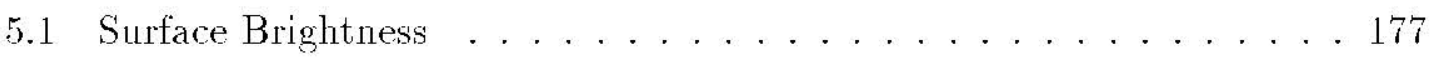

Conclusions

References 


\section{List of Figures}

0 Histograms of linewidths for the Sample . . . . . . . . . . -16

1 KPNO Filter Transmission Curves .............. 19

2 Comparison with Surface Photometry from Jitcrature . . . . . . 37

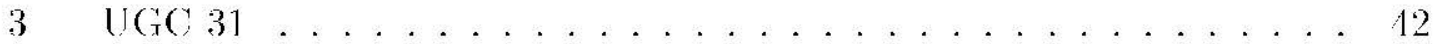

$4 \quad \mathrm{UGC} 63 \ldots \ldots \ldots \ldots \ldots \ldots \ldots \ldots$

$5 \quad \mathrm{UGC} 300 \ldots \ldots \ldots \ldots \ldots \ldots \ldots \ldots$

6 Karachentseva $10 \ldots \ldots \ldots \ldots \ldots \ldots$

7 Karachentseva $10(\mathrm{Jan} 93) \ldots \ldots \ldots \ldots$. . . . . . . . . . . .

$8 \quad \mathrm{UGC}_{1981 \ldots \ldots \ldots \ldots \ldots} \ldots \ldots \ldots \ldots \ldots$

$9 \quad \mathrm{UGC} 1981(\operatorname{Jan} 93) \ldots \ldots \ldots \ldots \ldots$

$10 \quad \mathrm{UG}_{\mathrm{C}}, 2017 \ldots \ldots \ldots \ldots \ldots$

$11 \quad \bigcup G(2017(\operatorname{Jan} 93) \ldots \ldots \ldots \ldots$

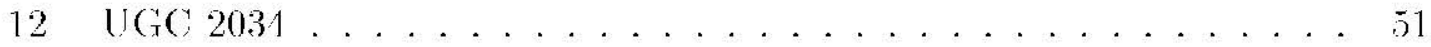

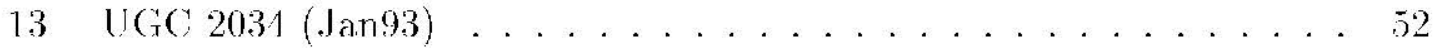

$14 \mathrm{UGC} 2053 \ldots \ldots \ldots \ldots \ldots \ldots \ldots \ldots$

15 Karachentseva $37 \ldots \ldots \ldots \ldots \ldots \ldots$

16 Karachentseva $37(\operatorname{Sep} 91) \ldots \ldots \ldots \ldots \ldots$

17 Karachentseva $37 \mathrm{C}($ Sep91) . . . . . . . . . . . . 56

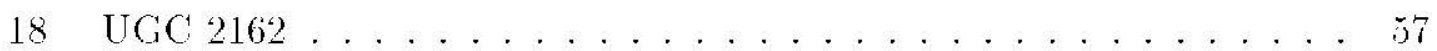


$19 \mathrm{UGC} 2162(\operatorname{Tan} 93) \ldots \ldots \ldots \ldots . \ldots \ldots$

$20 \quad \mathrm{UGC}_{3212} \ldots \ldots \ldots \ldots \ldots \ldots \ldots \ldots$

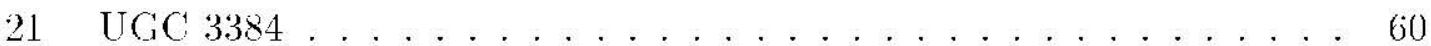

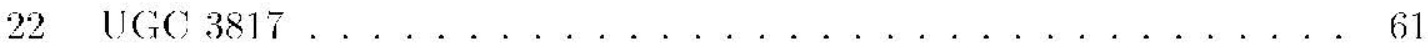

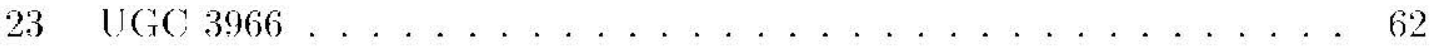

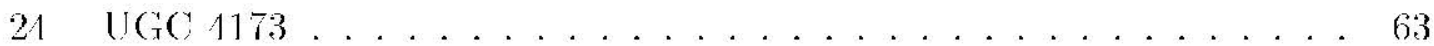

$25 \mathrm{UGC} 4173(\mathrm{Jan} 93) \ldots \ldots \ldots \ldots \ldots \ldots$

$26 \mathrm{UGC} 4204 \ldots \ldots \ldots \ldots \ldots \ldots \ldots \ldots$

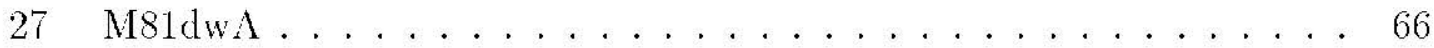

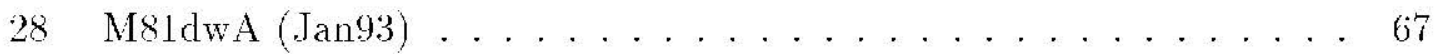

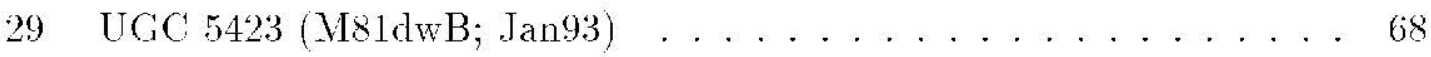

$30 \quad \mathrm{UGC} 5706 \ldots \ldots \ldots \ldots \ldots \ldots$

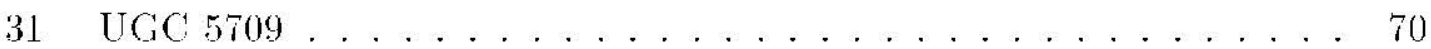

$32 \cup\left(\begin{array}{l}\text { UC } 6151 \ldots \ldots \ldots \ldots \ldots \\ 71\end{array}\right.$

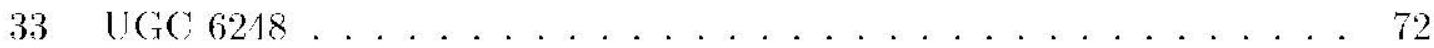

$31 \mathrm{UGC} 6596 \ldots \ldots \ldots \ldots \ldots$

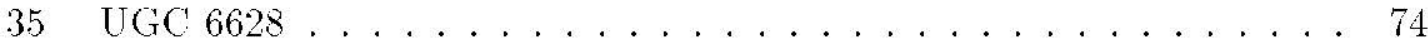

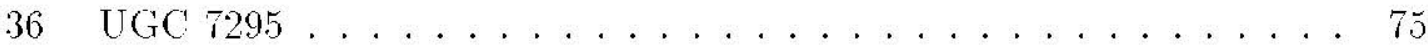

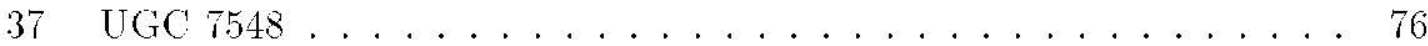

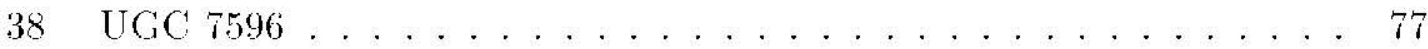

$39 \quad \mathrm{UGC} 7608 \ldots \ldots \ldots \ldots \ldots \ldots . \ldots \ldots \ldots$

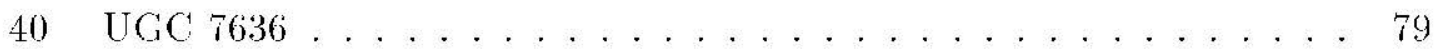

$41 \quad \mathrm{UGC} 7684 \ldots \ldots \ldots \ldots \ldots \ldots$

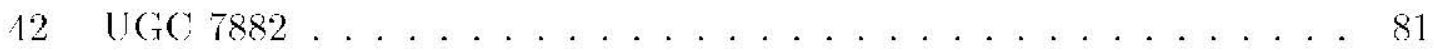

$13 \mathrm{UGC} 8091(\mathrm{GR} 8) \ldots \ldots \ldots \ldots 2 \ldots \ldots \ldots$ 
$\mathrm{UGC} 8683 \ldots \ldots \ldots \ldots \ldots$

$\mathrm{UGC} 8760 \ldots \ldots \ldots \ldots . \ldots \ldots . \ldots \ldots$

$\mathrm{UGC} 8833 \ldots \ldots \ldots . \ldots \ldots$

49 UGC $10031($ B-May85) . . . . . . . . . . . . . 88

$50 \quad$ UGC $10058($ B-May85) . . . . . . . . . . . . . . 89

$51 \quad \mathrm{UGC} 10290(\mathbf{B}-\mathbf{M a y} 85) \ldots \ldots \ldots$. . . . . . . . . . . 90

52 UGC $10376($ B-May85) . . . . . . . . . . . . . . . 91

$53 \quad \mathrm{UGC} 10669(\mathbf{B}-\mathrm{May} 85) \ldots \ldots \ldots \ldots 2$

$54 \quad \mathrm{UGC}_{12082}(\mathrm{Sep} 91) \ldots \ldots \ldots \ldots \ldots$

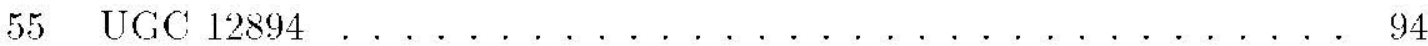

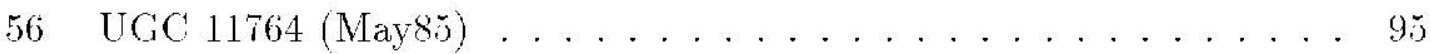

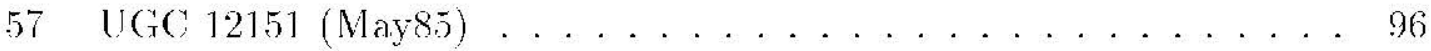

$58 \quad \mathrm{UGC} 772$ and $\mathrm{UGC} 1171 \ldots \ldots \ldots \ldots$

$59 \mathrm{UGC} 3860(\mathrm{Oct} 90)$ and $\mathrm{LCGC} 6628(\mathrm{Apr} 93) \ldots \ldots . \ldots 9$

60 UGC 7608 and UGC 8201 (both $\Lambda$ pr 93 ) $\ldots \ldots \ldots \ldots$

$61 \mathrm{UGC} 10031$ and UGC 10058 (both May85) . . . . . . . . . . . . 100

62 UGC 10290 and UGC 10376 (both May85) . . . . . . . . . . . . 101

$63 \mathrm{UGC} 10669$ (May8s) . . . . . . . . . . . . . . 102

$64-71$ Color Profiles and Disk Parameters . . . . . . . . . . 104

$72-74$ HII Region Size Distribution . . . . . . . . . . . . 113

$75-7$ HII Region Radial Distribution . . . . . . . . . . 116

7880 HII Region Azimuthal I)istribution . . . . . . . . . . . . 119

$81 B$-band T'ully Fisher Relation ................... 115 
$82 \quad I$-band Tully-Fisher Relation . . . . . . . . . . . 145

83 Residuals from Tully-Fisher Relation . . . . . . . . . . . . 147

84 Modified Tully-Fisher Relation . . . . . . . . . . . 153

85 Virial Mass/Luminosity I)iagrams . . . . . . . . . . . . . 151

86 literature Virial Mass/Luminosity I)iagram . . . . . . . . . . 157

87 Virial Mass/Luminosity I)iagram for Gaussian I)warfs . . . . . . . 159

88 Virial Mass/Luminosity Diagram for DII Sample . . . . . . . . . . . . 160

89 Virial Mass/Luminosity Diagram for DII Sample (LSBs excluded) . . 162

$90 \quad I$-band Virial Mass/Luminosity Diagram . . . . . . . . . . . . . 164

91 Surface Brightness/Linewidth Relation . . . . . . . . . . . 167

92 Color/Linewidth Relation . . . . . . . . . . . . 169

93 Radius/Luminosity Relation ................... 178

94 Average Surface Brightness versus Absolıte Magnitude . . . . . . 180

95 Central Surface Brightness versus Absolute Magnitude . . . . . . . 182

96 Disk Scalc Length versus Absolute Magnitude . . . . . . . . . . . 181 


\section{List of Tables}

1 Jata for Sample of I)warf Irregulars . . . . . . . . . . 16

2 Obscrving Run Information . . . . . . . . . . . . 19

3 Obscrving $\log \ldots \ldots \ldots \ldots \ldots$

4a. $2.1 \mathrm{~m}$ Photometric Calibration . . . . . . . . . . . . . 29

$4 \mathrm{~b} \quad 0.9 \mathrm{~m}$ and $4 \mathrm{~m}$ Photometric Calibration . . . . . . . . . . 30

5 Uncorrected Disk Parameters and Magnitudes . . . . . . . . . . . 129

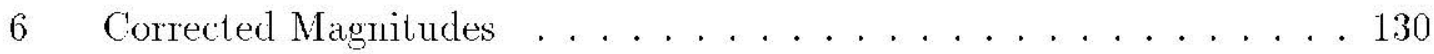

7 Distance Dependent Quantities ............... 134

$8 \quad R$-band Photometric Results ........................

9 Mass/Luminosity Fitting Results for Sample . . . . . . . . . . 165 


\section{Abstract}

CCD $B$ - and $I$-band surface photometry is presented for a sample of 51 dwarf and low surface brightness galaxies. The main selection criterion was their extremely narrow HI linewidths $\left(\Delta V_{20}<100 \mathrm{~km} \mathrm{~s}^{-1}\right)$, chosen in order to examine the Tully-Fisher (TF) relation between linewidth and absolute magnitude at the low-luminosity end. In this regime, a substantial fraction of the linewidth is attributable to turbulent rather than rotational motion. Therefore, it is not surprising that the original TF relation, an expression of the scaling relation between mass (from the linewidth due to rotation) and luminosity, does not hold in this regime.

If we assume the linewidth is due to both turbulent and rotational motion, we obtain a mass for the dwarfs which does correlate with luminosity. However, the relation is distinct from the TF relation for spirals, and indicates the presence of an increasing amount of dark matter at low luminosities. We find that the locus of dwarf galaxies in the mass/luminosity plane is well fit by the theoretical prediction of Dekel \& Silk [1986, ApJ, 303, 39], $M / L \propto L^{-0.37}$, where $M / L$ decreases with increasing $L$, while the spirals follow a relation in which $M / L$ increases with increasing $L$ $\left(M / L \propto L^{0.2}\right)$, which corresponds to the observed slope $(\sim 7)$ of the TF relation for spirals. For dwarfs fainter than $L_{B} / L_{\odot} \sim 10^{7}$, the observed $M / L$ is $\sim 20$, while it drops to unity for $L_{B} / L_{\odot} \sim 10^{9.5}$.

The Dekel-Silk relation arises in low mass systems with massive dark halos which undergo extensive mass loss due to supernova-driven winds. The halo allows the galaxy to remain bound even as most of the gas is removed, drastically reducing the rate of star formation. The trend towards higher $M / L$ at lower $L$ is the result of the lower escape velocity of the less massive systems, allowing the gas to be removed more efficiently.

The same relation has previously been seen to hold for dwarf Spheroidal galaxies, providing further evidence for a common evolutionary history of these two types of dwarf systems, radically different from "normal" early- and late-type galaxies. 


\section{Chapter 1}

\section{General Introduction}

\subsection{Dwarf Galaxies}

Dwarl Galaxies are the most common type of galaxy in the Universe. IIowever, "normal" galaxies dominate galaxy catalogs except in the few distance-limited catalogs (c.g. Kraan Korteweg 1986; 'lully 1988; Schmidt \& Boller 1992) where their intrinsic faintness (absolute $B$ magnitude, $M_{\mathrm{B}} \lesssim-16$ ) is not a factor. 'The growing realization of the astrophysical importance of dwarfs has led recently to increased numbers of studies of these objects.

This interest is based in part on the observed excess of faint blue galaxies at intermediate redshift as compared to the local luminosity function (cf. Tyson 1988; Broadhurst, Ellis, \& Shanks 1988; Ferguson 1992, Babul \& Rees 1992). This can be interpreted as evidence for a large population of star forming dwarls which have now 
faded away. In addition, there is increasing evidence that accretion and mergers of dwarf galaxies may play a large role in galaxy formation (Schweizer 1992).

Dwarf galaxies are found in all environments although there does appear to be some segregation based on type (Binggeli, Tarenghi, \& Sandage 1990). Gas-rich dwarl irregulars (dIs) are more likely to be found outside of clusters, often in loose groups. The most extreme star-lorming dwarfs are the blue compact dwarls (BCDs).

Dwarf Ellipticals (dEs), on the other hand, tend to be found in clusters of galaxies or as close companions to massive galaxies: dfis comprise $\sim 75 \%$ of the members of the Virgo cluster. In this work, we shall limit our scope to the gas rich dwarf irrcgulars.

In the remainder of this introduction, we discuss the following areas in which dwarf galaxics arc of significant astrophysical interest. I)warf irregulars present a simplified setting within which to study star formation. They often appear optically as little more than intergalactic H II regions, where star formation can be studied in isolation, without complicating factors such as spiral density waves. Similarly, the primordial (low metallicity and high gas content) state of dwarls makes them suitable to studies of galaxy formalion.

The low (baryonic) masses of dwarls allow strong constraints to be placed on the nature of the dark maller in the halos of galaxies (Tremaine \& Gunn 1978). The large numbers of dwarfs makes them useful as test particles for cosmological studies.

Finally, due to the smaller masses and lower rotation velocitics of dls as compared to spirals, the Tully Fisher (TF) relation can be tested in the extreme case of 
turbulence-dominated motion. Investigation of the TF relation in this regime should help to shed light on the exact origin of the relation.

\subsection{Star formation}

A simple phenomenological model of star formation, called stochastic self-propagating star formation (SSPSF) has been developed and applied to the dIs (Gerola, Seiden \& Schulman 1980). This model appears to fit observations lairly well in a qualitative way (Hunter \&. Gallagher 1985), while the physical processes which drive star formation remain poorly understood. In low mass dls the SSPSF model predicts distinct bursts of star formation. 'This is consistent with the picture of BCI)s as dIs in the middle of a burst (Thuan 1986).

Due to their extreme physical characteristics, $\mathrm{dI}$ s can also be used to directly study the conditions which lead to star formation. For example, is there a "universal" minimum H I density required before star formation can occur, similar to the relation found by Kennicutt (1989) for spirals? Studies show that the III content of dIs appears to increase with respect to the total mass as the mass decreases (StaveleySmith, Davies, \& Kinman 1993). IIowever, below $M_{\mathrm{B}} \sim-12$ there appears to be a dramatic rise in the mass to light ratios for dIs (Frecman 1987; Lo, Sargent \& Young 1993), which corresponds to the $\mathrm{HI}$ mass, $M_{\mathrm{HI}}$, just dropping below $10^{7} M_{6}$. Further study is necded before it will be known if the onset of star formation corresponds to a minimum gas density. Moreover, it is not presently understood how such low mass systems which lack rotational support can avoid collapsing and forming stars 
for periods $\gtrsim 10^{9}$ years, which is the time interval between bursts in a BCD (Thuan $1991)$

The current star formation rate (SFR) in a galaxy can be estimated from observations of the II $\alpha$ luminosity, $L(\mathrm{II} \alpha)$, since most of the stars providing the ionizing radiation are massive and therefore short lived. In addition, ultraviolet spectra from IUE observations allow the massive star population to be directly investigated (Fandli, O'Connell, \& Thuan 1988). Broadband colors, on the other hand, are dependent on both age and metallicity, and it is therefore difficult to obtain more than an estimate of the star formation history of a galaxy from such data. 'l'hese broadband colors can be combined with the evolutionary models of Larson \& Tinsley (1978) or, more recently, Bruzual \& Charlot (1993) to provide an estimate of the age of the most recent burst of star formation, as well as the age of the underlying old stellar population.

Kennicult (1989) has found a star formation law in disk galaxies in which star formation was found to occur in gas very close to a threshold surface density. It would be interesting to compare the gas densities in regions of star formation in dwarf galaxics to sec if similar conditions are found to apply. Lo et al. (1993) find that the HI surface densitics in a sample of nine faint, largely non-rotating dwarfs significantly execed the Kennicutt critical threshold density. The intrinsic faintness and paucity of $\mathrm{H} \alpha$ emission in these dwarfs, indicates little star formation in these systems, perhaps due to the lack of some sort of "trigger" such as differential rotation.

Van der Hulst et al. (1993), on the other hand, have found that in a sample of LSB disk galaxies, only small areas of the HI disk exceed the critical HI threshold. 
which agrees at least quantitatively with the Kennicutt model, in which the marked lack of star formation in LSB galaxies is interpreted as being due to the low HI surlace density in the disk. These two results, one for turbulence-supported, faint dwarl galaxies, and the other for rotationally-supported LSB galaxies, point to the critical role played by differential rotation versus stochastic cloud collisions in the process of star formation.

Any cpisode of massive star formation in such low mass objects would very likely Iead to the neutral gas being blown out from the stellar disk because of their low cscape volocity. Ionizing photons from (OB associations and winds from supcrnovac would give rise to holes in the HI distribution observed in the dIs Holmberg II (Puche et al. 1992), as well as Holmberg I and M81dwA (Westpfahl \& Puche 1994). Broadline components of $\mathrm{H} \alpha\left(\Delta v \sim 1000-3000 \mathrm{~km} \mathrm{~s}^{-1}\right)$ seen in several BCDs observed by Thuan el al. 1994, as well as the elongated X-ray emitting structure around VII Zw 403 (Papaderos el al. 1994), provide additional evidence for mass outhlow in dwarls. This may lead to a self-regulation of the star formation rate in the entire galaxy: no further episode of star formation can occur until the massive stars have died and the the neutral gas is allowed to cool and collapse into the center of the gravitational potential (Hunter, Hawley \& Gallagher 1993). If the gas is entirely lost by the galaxy, star formation will be essentially quenched (Dekel \& Silk 1986).

Any detailed determination of the stellar populations of the individual galaxies requires the application of the techniques of population synthesis. This involves comparing the combined galaxian spectral energy distribution to a stellar library, in order to determine the approximate composition of the galaxy by stellar type. 
Hunter \& Gallagher (1985) find evidence of low but steady star formation rates when modeling brighter dwarf irregulars. More detailed synthesis techniques, drawing on a stellar spectral library in the LV, have been employed by Fanelli, O'Connell \& Thuan (1988) for modeling the star formation histories of several BCDs. They find clear evidence of episodic bursts of intense star formation activity in these objects.

\subsection{Galaxy Formation}

Because of the high gas content, low star formation rate, the low metallicity and the lack of spiral structure, present day dIs may closely approximate conditions which existed in larger galaxies while they were forming. Of course, the free fall collapse leading to the formation of the bulge in spirals does not occur in dwarfs; (Sandage 1986). In spite of this difference, the presence of a large number of dwarfs in various stages of evolution that are within easy observational reach make them objects worthy of detailed study.

The low metallicity of the II II regions in dIs also lend themselves to a study of the chemical evolution of galaxies (Peimbert 1985). In BCDs, a primordial helium abundance, $Y$, can be obtained by extrapolating the $Y$ vs $O / I I$ relation to zero metallicity (Pagel ct al. 1992; I7otov ct al. 1991). 


\subsection{Dark Matter}

The missing-Inass problem first pointed out by Zwicky (1933). has remained a problem in that we are still uncertain of the nature of the dark matter which leaves its dymannical signature in the rotation curves of spirals and in the motions of clusters of galaxics. Because the number density of dwarf galaxics increases so stceply at. low luminosity, it is very important to determine how much, if any, dark matter is prescnt in dwarfs. It is possible that while dwarfs contribute a negligible amount to the total luminosity, they may contribute significantly, or even dominate in the mass distribution in the universe.

Observations to detect dark matter in dIs have been undertaken by several groups (Carignan, Beaulieu \& Freeman 1990; Lo et al. 1993). They combine VLA 21cm observations with optical data to construct models of the III distribution in the dwarls and the overall mass-to-light ratios. They find that the extreme dIs with $M_{\mathrm{B}} \sim-10$ and $M_{\text {virial }} \sim 10^{7} M_{5}$ seem to be embedded in a dark mat ter halo. Values for $M / L$ as high as $\sim 25$ were reported in the extremely low luminosity dwarf I.GS 3 $\left(M_{\mathrm{B}} \sim-9.2\right)$. 'T'his low limit on the presenec of a dark halo, along with similar work for dts (Mateo 1993), effectively rules out massive neutrinos or other dynamically "hot" low-mass particles as the source of the dark matter. 


\subsection{Cosmology}

Biased galaxy formation (Rees 1985), in which "Iıormal" galaxies arise from 2-3 $\sigma$ peaks in the density fluctuations in the early universe, explains voids as regions in which the mean density was slightly below the average and no peaks large enough to form normal galaxies occurred. It is expected that dwarf galaxics would have formed from much smaller fluctuations $(\sim 1 \sigma)$, and they should therefore populate the voids obscrved in redshift surveys (1)ekel \& Silk 1986).

Several H I surveys of dwarf galaxics were carried out in order to test just this hypothesis (Thuan et al. 1991, and references therein). Although a few faint galaxies have been found to lie within voids (Salzer, Hanson, \& Gavazzi 1990), nearly all of these surveys found that the dIs follow the void boundaries in the same way as the larger galaxies. These observations place restrictions on the biased galaxy formation theory. Interestingly, Salzer (1989) and Salzer \& Rosenberg (1994) find that active, emission line galaxies (ELGs) are sometimes lound in voids. Recently, Pustil'niik et al. (1994a and $b$ ) find that $\sim 20 \%$ of the BCI)s in the Second Byurkan Survey are found in voids.

\subsection{The Tully-Fisher Relation}

It has long been known that there is a strong correlation between the rotational velocity and the luminosity of spiral/irregular galaxics. However, it was not until the work of Tully \& Fisher $(1977)$ that the usefulness of this correlation for the determination 
of extragalactic distances was fully appreciated. Therefore, this luminosity-linewidth relation is often referred to as the Tully-Fisher (TF) relation.

The rotational velocity of a disk galaxy can be obtained from observations of the global II I line profile lor the galaxy. This velocity implies a value for the luminosity of the galaxy through the TF relation; therelore by measuring the apparent magnitude of the galaxy, one can determine the distance modulus. The line width must be corrected for the effects of inclination, and the galactic magnitude must be corrected for internal and galactic extinction. 'This leads to a relation of the form,

$$
B_{T}^{O}=a \log \left(\Delta V_{20}^{0}\right)+b
$$

where $B_{T}^{O}$ is the total absolute $B$ magnitude corrected for internal and external extinction, $\Delta V_{20}^{0}$ is the II I 21 cm linewidth at $20 \%$ of peak flux corrected for the inclination of the galaxy and $a$ and $b$ are constants, determined through observations of a large group of calibrator galaxies with reliable distances determined by other means (e.g., the Cepheid period luminosity relation).

Most of the carly calibration and application of the 'T' $\mathrm{F}$ ' relation was carrice out 11sing $B$ magnitudes, as these were the most commonly available magnitudes. However, a strong Hubble type dependence was found to exist for the $B$ ' Tully Fisher relation (Roberts 1978: Rubin et al. 1985), because the $B$-band is a strong tracer of the young stellar population in a galaxy and this varies with type at a constant galaxian mass. The uncertainty in the (large) correction due to internal extinction in $B$ further increased the dispersion of the TF relation, which is observed to be $\sim 0.35$ Inag (Pierce \& Tully 1988). 
This difficulty was remedied by using infrared $H$-band magnitudes (Aaronson, Huchra \& Mould 1979). Since the infrared luminosity is dominated by late-type giants, it is less subject to young star formation than the $B$ magnitude. The extinction in $I I$ is only $10 \%$ of that in $B$, so the correction for internal extinction introduces less uncertainty in the infrared TF relation. This was reflected in a significantly reduced dispersion in the infrared 'T'F' relation. 'The dispersion in the $H$ (as well as the $R$ and /) band relation is $\sim 0.25 \mathrm{mag}$ (Picrec \& Tully 1992).

Picres and Tully (1988) determined 'T'F' relations for three different bands, B, $V$, and $I$, and found that the dispersion for the $I$ band was $\sim 25 \%$ smaller than that in the $B$-band (most of the remaining dispersion may have been due to depth effects in the clusters they were using). The $I$-band has since become one of the most important bands for TF-type studies (e.g. Lu 1993) for several reasons. First, the limits in the sensitivity of infrared arrays and the brightness of the infrared sky make it exceedingly difficult to obtain accurate $I I$-band magnitudes for faint, extended galaxies. Second, the $I$-band CCD data also has the advantage over the $B$ band of allowing smaller corrections for the (uncertain) Galactic and internal extinction. Finally, the I band traces the older stellar population, and therefore the mass, of the galaxy better than the $B$ band, which contributes to the tighter relationship found by Pierce \& Tully. This sensitivity to the older stellar population also leads to a better estimate of the inclination from surface photometry.

While the TF relation is essentially an empirical relation (which must therefore be carefully calibrated in each wavelength in which it is employed), there is some physical basis for the relation, at least for spiral galaxies. The following justification 
is outlined in Aaronson, Huchra \& Mould (1979).

For a spherically symmetric rotating galaxy, the balance of gravitational and centrifugal force implies that the total mass $M_{T}(r)$ contained within $r$ is $M_{T}(r) \sim$ $r V^{2}(r)$, while constant surface brightness implies $L \sim r^{2}$. Assuming a constant massto-light ratio $(M / L)$, the combination of the two previous relations gives $L \sim V_{\text {mas }}^{4}$ (which is equivalent to the Faber-Jackson relation for elliptical galaxies). This yields a slope of 10 for the 'I'F' relation ( $\alpha=10$ in equation 1.1$)$ which is roughly the slope found for the $H$ band relation.

A major problem with this simple picture is the fact that the mass to light ratio is observed to increase dramatically for low luminosity systems (Picrec 1991; lo et al. 1993), yet with the scarce existing data, these galaxies appear to obey the same TF relation as the more massive galaxies which have a nearly constant $M / L$. These dwarfs appear to be dark matter dominated; however, the visible matter must be tightly coupled to the dynamical mass, if the TF relation is to hold. $A$ similar situation is seen in the disk-halo "conspiracy" in spiral galaxies (Sancisi \& van Albada 1987).

Very lew galaxies at the low-luminosity end have sufficiently accurate magnitude data to constrain the TF relation in this regime. The major motivation for this thesis was to obtain observations for a sample of such dwarfs, in order to investigate the precise character of the 'l'F' relation at very low luminositics. 


\subsection{Outline of the Thesis}

In order to investigate the optical properties and Tully-Fisher relation for extreme dwarl galaxies, a sample of 50 dwarl irregular galaxies with very narrow III linewidths (dominated by turbulent rather than rotational motion), was observed in both the $B$ and $I$ bands. From these observations we shall investigate the I band 'liblly Fisher relation (as well as the less reliable, but more widely used $B$ band relation) in the low luminosity regime.

I'he thesis is organized in the following way. In Chapter 2, the observations and reductions are detailed and the surface photometry is presented. In Chapter 3, the corrections to absolute magnitude, from our surface photometry and H l linewidth, from the literature, for reddening, dust and inclination are discussed. These results are used in Chapter 4 to derive a Tully-Fisher relation for small $\Delta V_{20}^{0}$ and the scatter in the relation is analyzed. $A$ modified version of the Tully-Fisher relation is suggested for dwarl galaxies. In Chapter 5, the structural parameters and global propertics of the dwarfs are cxamined and compared with ISSB and normal spirals. Finally, a summary of the results is presented. 


\section{Chapter 2}

\section{CCD Surface Photometry of Dwarf Galaxies}

\subsection{Sample Selection}

The galaxies in this sample are very low luminosity dwarf galaxies selected by their III line width, $\Delta v_{20}$, III velocity, and magnitude. They are drawn almost exclusively Crom the sample of 1557 Uppsala General Catalogue (UGC; Nilson, 1973) dwarl and low surlace brightness galaxies which were detected in III by Schneider $t$ l al. (1990, 1992). 'Their sample included all galaxies in the UGC classified with a Hubble type of Se Irr or later (or a de Vaucoulcurs class of $\mathrm{Sd} d \mathrm{dm}$ or later), with the majority chosen for our subsample classificd as dwarfs, which Nilson catcgorized as "objects with (1) very low surface brightness and (2) little or no central concentration of light on the red prints". 
Galaxies were chosen which had a $\Delta v_{20}<100 \mathrm{~km} \mathrm{~s}^{-1}$ and a $v_{0}<1500 \mathrm{~km} \mathrm{~s}^{-1}$, with a sufficiently faint $\mathrm{m}_{\mathrm{Pg}}$ (from '/wicky ct al. 1960 1968, or from Nilson's own very uncertain visual estimates) to qualify as a dwarf under the definition of 'lammann (1980): $M_{B} \gtrsim-16$. For galaxics not observed by Schneider et al. (1990; 1992), H I data was taken from Huchtmeier \& Richter (1989).

The major and minor axis ( $a$ and $b$ from Nilson) and the Hubble classification from Nilson were used in an attempt to exclude face-on spirals from the sample. Finally, the Palomar Observatory Sky Survey (POSS) prints were visually inspected in order to include only dwarls in this sample. These were usually found to be galaxies of low surface brightness with superimposed irregular patches of star formation. The III profile shape was used to select a large number of galaxies with Gaussian profiles. This should restrict the sample to galaxics which lack rotational support (after excluding the face on spirals).

In addition to the low redshift, narrow width dwarfs in the sample, several low surface brightness (LSB) galaxics at larger redshift, and/or with larger line widths were included in the sample, in order to allow overlap and comparison with other Tully-Fisher relation studies.

In Table 1, the basic data for the sample are presented. The UGC number (or other name) is listed (marked by a $c$ if it is an LSB galaxy), followed by the coordinates, the heliocentric velocity $v_{\infty}$, the III line full widths at hall power and at $20 \%$ maximum, and the III flux corrected for beam size, all from Schneider et al.. The profile shape is then classified as a Gaussian (G), probable Gaussian (G?), 
probable double-horned (D?), or double-horned (D). Galaxies for which the signalto-noise of the HI observations was too poor to allow an accurate classification were all placed in the two intermediate categories (D? and G?). The results discussed in Chapters 4 and 5 are based only on the galaxies which could be definitively classified as either D or G. Finally, the optical blue dimensions, IIubble Type and photographic: magnitude taken from the L: $G_{T}($ are listed.

In Figure 0 (immediately after 'lable 1) a histogram of the linewidth distribution is shown for the narrow-lined dwarfs in (a), as well as for the sample as a whole in (b).

Five of the seven galaxies for which $B$ and $R$ images had been obtained on the May 85 run (before the present project was fully conceived) were included for $I$-band observations, although they did not strictly satisfy the above selection criteria. All seven of these galaxies are marked by an $r$ in Table 1.

Finally, several galaxies were included because of their scientific interest. UGC 8091 (=GR8, DDO 155) was included to allow comparison with the surface photometry of Carignan, Beaulieu \& Freeman (1990). M81dwA was included because of the earlier work of Sargent, Sancisi \& Lo (1983) which indicates that this dwarf is probably not supported by rotalion. Kara 10 and 37 were chosen from the list of dwarf galaxics of Karachentscva (1968) which had single dish observations from Schneider \& Thuan (1989). K10 qualifies as a narrow lined dwarf as described above, but K3T has a large, single-peaked $\Delta v_{20}$, and was thought to be of interest. Subsequent VLA observations which we made of 10 galaxies in the sample, show that the unusual HI profile of $\mathrm{K} 3 \mathrm{i}$ is actually caused by two galaxies, K37 and IC342dw6 
Table 1

Data for Sample of Dwarf Irregulars

\begin{tabular}{|c|c|c|c|c|c|c|c|c|c|c|}
\hline Cialaxy & \multicolumn{2}{|c|}{$(1950.0)$} & $\begin{array}{c}v_{0} \\
(\mathrm{kmm} / \mathrm{s})\end{array}$ & $\begin{array}{c}\Delta \mathrm{v}_{\mathrm{s} 0} \\
(\mathrm{kmm} / \mathrm{s})\end{array}$ & $\begin{array}{c}\Delta \mathrm{v}_{20} \\
(\mathrm{kmrl} / \mathrm{s})\end{array}$ & $\begin{array}{c}\text { Flux } \\
\left(J_{y} \cdot \operatorname{lsm} / \mathrm{s}\right)\end{array}$ & $\begin{array}{l}\text { Frofile } \\
\text { Shape }\end{array}$ & $\begin{array}{c}a \times b \\
(\prime)\end{array}$ & $\begin{array}{l}\text { Hubble } \\
\text { lype }\end{array}$ & $\begin{array}{l}\mathrm{m}_{y, q} \\
(\mathrm{~L} w)\end{array}$ \\
\hline L 00031 & $0002 \quad 18.2$ & +165150 & 1239 & 11 & 68 & 1.97 & (3) & $1.1 \times 1.1$ & TRER & 15.3 \\
\hline L:00063 & $000.5 \quad 16.0$ & +351118 & 687 & 39 & 39 & 3.52 & Ci & $1.0 \times 0.7$ & TRE & 15.6 \\
\hline t,00300 & 002729.7 & +031103 & 1189 & 27 & 11 & 1.10 & Gi & $1.1 \times 1.1$ & DWARF & 18. \\
\hline 100772 & 011106.0 & +003642 & 1263 & 43 & 66 & 5.10 & $G$ & $1.6 \times 1.4$ & LWRE IR & 17. \\
\hline Lo1171 & 013702.4 & +153851 & 874 & 29 & 51 & 1.53 & $G$ & $1.4 \times 1.3$ & DWAKF & 17. \\
\hline KARA10 & 014054.3 & +152627 & 921 & 35 & 52 & 3.64 & $\mathrm{G}$ & $\ldots$ & & $\ldots$ \\
\hline L01981 & 022743.1 & +004306 & 1546 & 31 & $4 \pi$ & 1.06 & G & $1.7 \times 1.5$ & DWART & 17. \\
\hline $\mathrm{L}, 02017$ & 022951.0 & +28.3700 & 1123 & $\ldots$ & 101 & 11.10 & $\mathrm{C}$ & $2 . \overrightarrow{5} \times 2.0$ & DWRF IR & 17. \\
\hline T:02034 & 023031.0 & +101826 & 752 & 11 & 66 & 31.10 & П? & $3.5 \times 3.0$ & DWRF IR & 1.3 .0 \\
\hline 102053 & 023131.0 & +293147 & 1174 & 55 & 64 & 29.00 & $G$ & $2.3 \times 1.3$ & DWR. IR & 15.7 \\
\hline $\mathrm{L} 02162$ & 023749.0 & +010047 & 1218 & 51 & $6 \overline{0}$ & 5.20 & G? & $2.0 \times 2.0$ & LWRF IR & 18. \\
\hline $\mathrm{K} \Lambda \mathrm{R} \Lambda 37$ & 032900.1 & +675502 & 1578 & 146 & 208 & 22.38 & $G$ & $\ldots$ & $\ldots$ & $\ldots$ \\
\hline $\operatorname{Los} 3212$ & 045.517 .9 & +710609 & 1398 & 49 & 72 & 1.33 & $\mathrm{G}$ & $1.2 \times 0.4$ & DWRF SP & 18. \\
\hline Cos3384 & 05.5 .525 .2 & +730700 & 1262 & 78 & 100 & 22.76 & D & $1.9 \times 1.9$ & DWRF SP & 16.0 \\
\hline to3s17 & 071906.6 & +151200 & 171 & $\ldots$ & 32 & 10.20 & G? & $2.0 \times 1.0$ & DWAFF & 17 \\
\hline $\mathrm{L}, 03860$ & 072150.2 & +105213 & 365 & 10 & $\overrightarrow{3 i}$ & 16.90 & G? & $1.8 \times 1.2$ & DWRF IR & 1.3 .5 \\
\hline L 03966 & 073801.1 & +401347 & 368 & 71 & 91 & 25.10 & $\mathrm{D}$ & $2.2 \times 2.0$ & LWRF IR & 16.0 \\
\hline 104173 & 075836.0 & +801600 & 1054 & $\ldots$ & 89 & 29.50 & $D ?$ & $3.3 \times 0.7$ & IRK & 15.7 \\
\hline c L04204 & 080125.2 & +560533 & 3053 & 130 & 179 & 3.50 & $\mathrm{D}$ & $1.2 \times 0.9$ & LWARF & 17. \\
\hline M81dwA & 081842.0 & +711136 & 262 & 19 & 37 & 4.20 & $\mathrm{G}$ & $\ldots$ & $\ldots$ & $\ldots$ \\
\hline Lo5423 & 100125.3 & +703627 & 466 & 47 & 67 & 3.39 & $\mathrm{G}$ ? & $1.3 \times 1.0$ & IRR & 15.3 \\
\hline L05706 & 102819.2 & +311535 & 1.166 & 33 & 18 & 3.99 & $\mathrm{G}$ & $1 . \overline{5} \times 1.2$ & DWRF IR & 18. \\
\hline$\therefore \operatorname{T} 05709$ & 102833.3 & +193830 & 6103 & 212 & $2 \pi 7$ & 2.82 & D & $1.3 \times 0.8$ & $\ldots$ & 1.3 .7 \\
\hline L06151 & 110316.0 & +200540 & 1241 & 27 & 41 & 6.40 & $(i ?$ & $1.8 \times 1.8$ & DWRF SP & 17. \\
\hline 106248 & 111015.9 & +102821 & 1156 & 30 & 48 & 1.94 & $G$ & $1.6 \times 1.3$ & LWR. IR & 18. \\
\hline 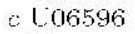 & 113508.1 & +562513 & 2379 & 154 & 177 & 10.88 & $\mathrm{D}$ & $1.2 \times 0.7$ & IRK & 15.5 \\
\hline L06628 & 113724.0 & +461200 & 901 & 41 & 56 & 24.70 & $\mathrm{G}$ ? & $3.5 \times 3.5$ & $\ldots$ & 14.6 \\
\hline c $\operatorname{Lu}_{2} 295$ & 121403.8 & +334334 & 6687 & 232 & 26.5 & 2.43 & $\mathrm{D}$ & $1.0 \times 0.25$ & $\ldots$ & 16.5 \\
\hline $\operatorname{Lo7548}$ & 122118.2 & +132708 & -86 & 30 & 11 & 2.37 & G? & $1.3 \times 0.5$ & TRE & 15.2 \\
\hline L 07596 & 122601.0 & +085154 & 165 & 25 & 12 & 0.28 & D? & $1.9 \times 0.7$ & DWAFIF & 1.3 .3 \\
\hline 107608 & 122618.0 & +433000 & 601 & 60 & 74 & 29.95 & $G ?$ & $4.0 \times 3.5$ & DWRF IR & 16.0 \\
\hline$\times 107636$ & 122728.3 & +081215 & 178 & 25 & 57 & 0.28 & $G$ & $1.2 \times 0.8$ & LWRF IR & 15.4 \\
\hline $\operatorname{Lo7} 684$ & 122948.4 & +183114 & 767 & 26 & 48 & 0.28 & G & $1.1 \times 0.7$ & & 15.4 \\
\hline c $\operatorname{Lot} 882$ & 124021.8 & +333334 & 6834 & 3.56 & 370 & 3.71 & D & $1.5 \times 0.2$ & Sc-IRR & 15.7 \\
\hline L08091 & 12.5609 .8 & +142912 & 163 & 31 & 47 & 8.40 & $\mathrm{G}$ & $1.1 \times 0.9$ & IRR & 1.5 .3 \\
\hline L-08201 & 130139.1 & +675816 & 202 & 12 & 68 & 36.00 & G? & $3.7 \times 2.5$ & DWRF IR & 1.1 .1 \\
\hline Tos683 & 13.1023 .0 & +395126 & 717 & 32 & 11 & 7.50 & $\mathrm{G}$ & $2.3 \times 2.3$ & DWRF IR & 1.5 .7 \\
\hline L 08760 & 134840.2 & +381548 & 273 & 38 & 45 & 9.60 & $G$ & $2.3 \times 0.6$ & DWRF IR & 15.4 \\
\hline Lo8833 & 135242.0 & +360500 & 303 & $\ldots$ & 38 & 5.30 & $G$ & $1.1 \times 0.9$ & $\mathrm{IRR}$ & 16.5 \\
\hline Lo9128 & 141337.9 & +231706 & 197 & 34 & .54 & 12.80 & $\mathrm{G}$ & $1.8 \times 1.6$ & DWRF IR & 15.3 \\
\hline c Log391 & 143313.0 & +593320 & 2090 & 119 & 134 & 7.94 & $\mathrm{D}$ & $1.8 \times 1.1$ & $\ldots$ & 15.5 \\
\hline r L10031 & 154454.6 & +614236 & 1099 & $\ldots$ & 71 & 7.00 & D & $1.7 \times 1.5$ & DWRF SP & 17. \\
\hline$r \mathrm{~L} 10058$ & 15.1817 .4 & +260.112 & 2276 & 117 & 13.5 & 5.83 & D & $1.2 \times 0.9$ & DWRF SF & 17. \\
\hline$r \quad-10290$ & 161159.1 & +005612 & 199.5 & 119 & 138 & 9.91 & D) & $2.0 \times 1.9$ & DWRF SF & 13.0 \\
\hline r L10376 & 162229.4 & +653300 & 3474 & 41 & 69 & 2.30 & $G$ & $1.5 \times 1.4$ & DWRF SP & 16.5 \\
\hline r L10669 & 170052.8 & +702136 & 681 & $\ldots$ & 52 & 3.00 & $G$ & $1.4 \times 1.4$ & LWAKF & 17. \\
\hline$r \quad \operatorname{Lu}_{11764}$ & 213045.0 & +074642 & 3717 & 73 & 107 & 3.76 & $\mathrm{D} ?$ & $1.0 \times 1.0$ & DWRF SP & 18.0 \\
\hline L12082 & 223152.7 & +323606 & 1081 & 67 & 83 & 31.60 & D? & $3.5 \times 3.0$ & DWRF SP & 15.6 \\
\hline$r$ L12151 & 223859.4 & +000824 & 1939 & 167 & 193 & 5.00 & D? & $3.0 \times 2.0$ & DWARF & 16.0 \\
\hline L:12894 & $23.57 \quad 18.0$ & +391254 & 592 & 34 & 57 & 6.75 & $\mathrm{G}$ & $1.0 \times 1.0$ & DWRF IR & 17. \\
\hline
\end{tabular}



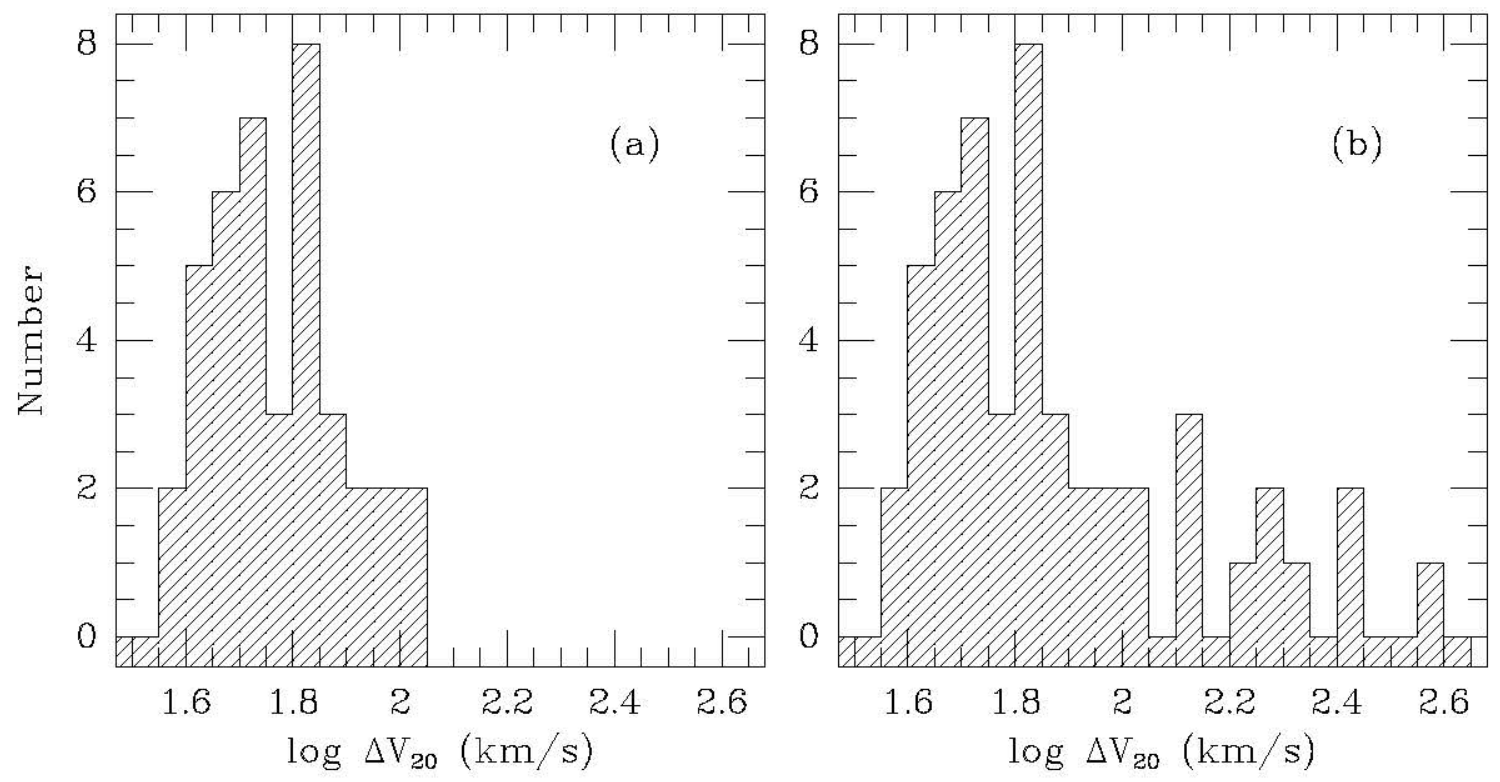

Figure 0: Histogram of Linewidths for the sample. (a) shows the distribution of linewidths for the narrow-lined galaxies $\left(\Delta V_{20} \lesssim 100 \mathrm{~km} \mathrm{~s}^{-1}\right)$. (b) shows the distribution for all the galaxies in the sample, listed in Table 1. 
(Börngen \& Karachentseva 1985), which appear from the VLA data to be spirals in the background of the IC 342 complex.

The HI data for UGC 7636 (marked by an asterisk in Table 1) actually applies to a cloud of III which has been removed from the dwarl by ram-pressure stripping by the hot $\mathrm{X}$-ray emitting gas around the giant elliptical $\mathrm{XGC} 4472$ in the Virgo Cluster (Patlerson \& Thuan 1992; McNamara el al. 1994). The dala for UGC 7636 are presented, but are cxcluded from the analysis in Chapters 1 and 5.

\subsection{Observations}

The $B, R$ and I photometric obscrvations of 51 dwarf and $I S B$ galaxics were obtained during several observing runs from 1985 to 1993 with different (CI) chips and telescope combinations. Specific details about the observing runs are given in Tables 2 and 3 . For each observing run, Table 2 details the number of galaxies observed and the filters used, as well as listing the telescope aperture and focal ratio, the CCD type, size and pixel scale, the field of view (FOV), the gain and read noise of the chip.

Table 3 gives the galaxy name, filters used, date of observations, filters used, number and length of exposures, seeing, airmass and sky conditions (photometric or some cirrus present).

Most of the these galaxies (38) were observed in the $B$-and $I$-bands in October 1990 and April 1991 at the $f / 7.5$ focus of the $2.1 \mathrm{~m}$ telescope at Kitt Peak Yational 
Observatory $^{1}$ (KPNO). The CCD camera used for these observations consisted of a Tektronix (TEK2/T5HA) chip. In addition, $T$ of the galaxies were observed in $B-$ and $R$-bands in May 1985 with the 4m telescope by Trinhı Thuan and Ken Mitchell (Thuan el al., 1992). In Sept. 1991, January 1993 and April 1993, 13 of the galaxies with the largest angular size (as well as those galaxies with images suffering from fringing problems, marked by an $f$ in Table 3: sec 32.3 ) were reobserved with a larger (U) chip at the $2.1 \mathrm{~m}$ or $0.9 \mathrm{~m}$ telescopes in order to image the entire galaxy and to include a sufficiently large area around the galaxy for proper sky subtraction. These subsequent observations were made by John Spitzak and Steve Schneider (Sep91); Rich Gelderman and Patterson (Jan93); and Trinh Thuan and Valentin Lipovetsky $(\Lambda \mathrm{pr} 93)$.

During the Oct 90 and Apr 91 runs the galaxies were imaged through the $B$ $\left(\lambda_{0}=4400 \hat{\Lambda}\right.$. FWIIM $\left.=1152 \hat{\Lambda}\right)$ and $I\left(\lambda_{0}=8205 \hat{\Lambda}\right.$, FWIIM=1851 $\left.\hat{\Lambda}\right)$ filters in the KPNO Mould filter system. Each observation was broken up into three separate exposures of 10 minutes in $B$ and 7 minutes in I and displaced from each other by $\sim 10^{\prime \prime}$ in Right Ascension or Declination, for a total of 30 minutes in the $B$ and 21 minutes in the / filter. 'I'hese slight displacements are useful in the subsequent. processing for removing bad pixels and cosmic ray cvents.

D)uring the May $85 \mathrm{Am}$ run, the Mould $B$ and Mould $K\left(\lambda_{0}=6467 \AA \mathrm{HWHM}=1108 \AA\right)$ filters were used, and the Mould $B$ filter was used for the Apr93 run. The Harris

\footnotetext{
${ }^{1}$ Kitt, Peak National Observatory, National (optical Astronomy (Observatories, operated by the Association of Universities for Research in Astronomy, Inc, under contract with the National Science Foundation
} 


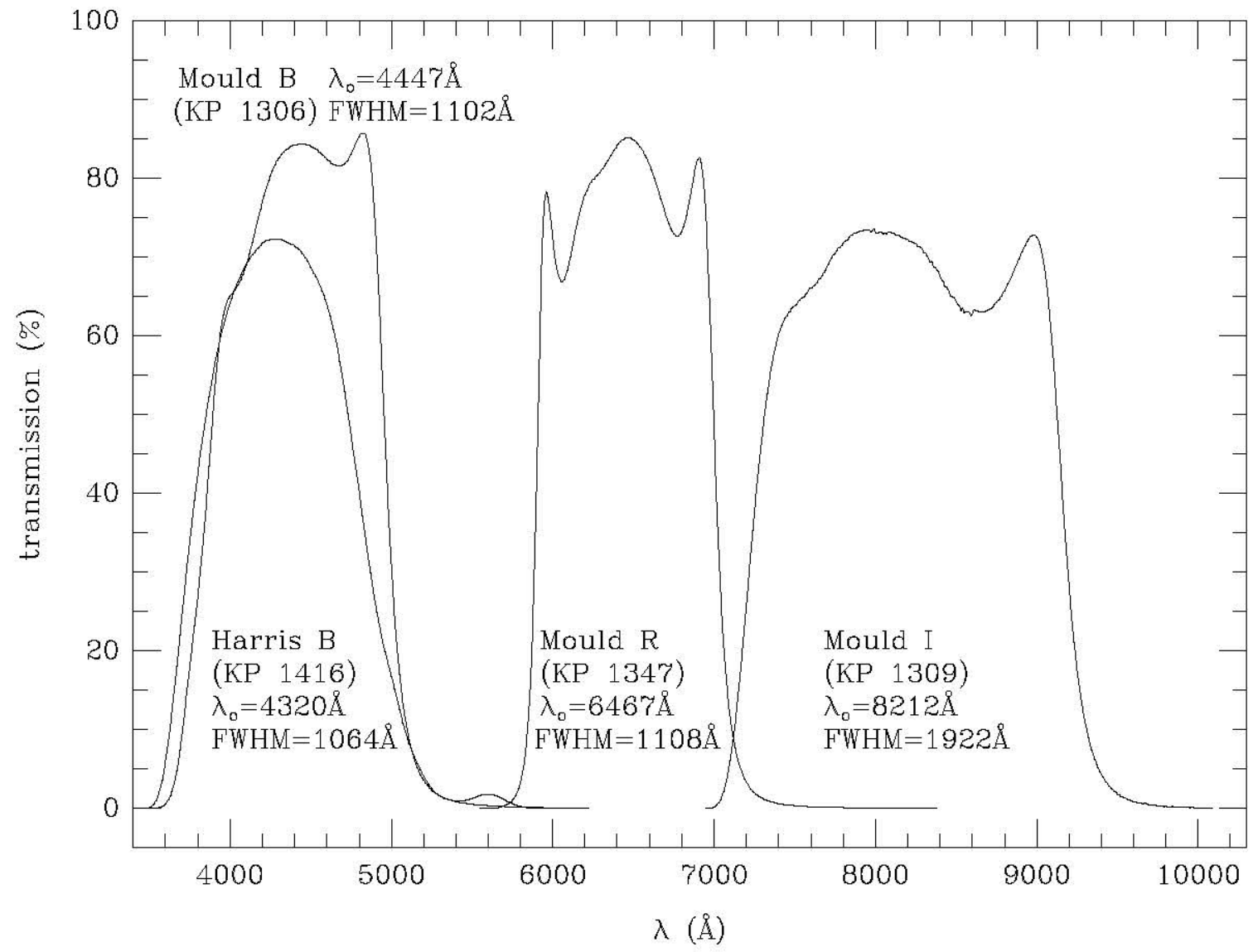

Figure 1: 'Iransmission curves, with $\lambda_{0}$ and FWHM, for the (C) F'ilters used in this work. F'rom KPNO manuals.

Table 2

Observing R.un Information

\begin{tabular}{|c|c|c|c|c|c|c|c|c|c|c|}
\hline Dato & \multicolumn{3}{|c|}{$\begin{array}{lrr}\text { c } & \# & \text { Band } \\
\text { (Galaxies Observed) }\end{array}$} & \multicolumn{2}{|c|}{$\begin{array}{c}\text { Tclescope \& CCD } \\
\text { (KFNO) }\end{array}$} & \multirow{2}{*}{$\begin{array}{c}\text { Sizc } \\
\text { (pixels) } \\
320 \times 512\end{array}$} & \multirow{2}{*}{$\begin{array}{c}\text { Scalc } \\
\left(\frac{\text { arczec }}{\text { pixel }}\right) \\
0.60\end{array}$} & $\begin{array}{c}\text { FOV } \\
\text { (arcmin) }\end{array}$ & \multirow{2}{*}{$\begin{array}{l}\begin{array}{c}\text { Gain } \\
\left(\frac{e^{-}}{A D t^{\prime}}\right)\end{array} \\
11.5\end{array}$} & \multirow{2}{*}{$\begin{array}{l}\text { RN } \\
\left(e^{-}\right) \\
79.0\end{array}$} \\
\hline $198.5 \mathrm{May}$ & 2223 & 7 & $B R$ & $4 \mathrm{~m}, \mathrm{f} / 2.8$ & RCA1 & & & $3.2 \times 5.1$ & & \\
\hline 1990 Od & $19-22$ & 16 & $B 1$ & $2.1 \mathrm{~m}, \mathrm{l} / 7.5$ & T5HA & $512^{2}$ & 0.34 & $2.9 \times 2.9$ & 3.25 & 7.5 \\
\hline $1991 \Lambda_{\mathrm{pr}}$ & $17-21$ & 29 & Bil & $2.1 \mathrm{~m}, \mathrm{l} / 7.5$ & T5HA & $512^{2}$ & 0.34 & $2.9 \times 2.9$ & 3.25 & 7.5 \\
\hline 1991 Sep & $13-16$ & 3 & $B I$ & $0.9 \mathrm{~m}, \mathrm{f} / 7.5$ & ST1K & $1021^{2}$ & 0.59 & $10.0 \times 10.0$ & 2.7 & 2.8 \\
\hline $1993 \mathrm{Jan}$ & 2324 & 8 & $B I$ & $2.1 \mathrm{~m}, \mathrm{f} / 7.5$ & TIKA & $1024^{2}$ & 0.30 & $5.1 \times 5.1$ & 1.7 & 3.5 \\
\hline $1993 \Lambda_{\mathrm{Pr}}$ & $18-20$ & 3 & $B$ & $0.9 \mathrm{~m}, \mathrm{l} / 7.5$ & $\mathrm{~T} 2 \mathrm{KA}$ & $1024^{2}$ & 0.68 & $11.6 \times 11.6$ & 7.0 & 4.0 \\
\hline
\end{tabular}


Table $\mathbf{3}$

Observing Log

\begin{tabular}{|c|c|c|c|c|c|c|c|}
\hline Galaxy & band & run & night & $\begin{array}{l}\text { cxp. timc } \\
\text { (\#xsec) }\end{array}$ & $\begin{array}{l}\text { sccing } \\
\text { arcsec }\end{array}$ & airmass & $\begin{array}{c}\text { sky } \\
\text { conditions }\end{array}$ \\
\hline \multirow[t]{2}{*}{ U31 } & $B$ & Oct90 & 22 & $3 \times 420.0$ & 1.7 & 1.08 & Photo \\
\hline & $I f$ & Oct90 & 22 & $3 \times 420.0$ & 1.4 & 1.05 & Photo \\
\hline \multirow[t]{2}{*}{ U 63} & $B$ & Oct 90 & 21 & $3 \times 600.0$ & 1.9 & 1.02 & Fhoto \\
\hline & $I f$ & Oct 90 & 21 & $3 \times 120.0$ & 1.1 & 1.01 & Fhoto \\
\hline \multirow[t]{2}{*}{ U300 } & $B$ & Oct90 & 20 & $1 \times 1800.0$ & 2.0 & 1.25 & Photo \\
\hline & 1 & $0 \cdot 190$ & 20 & $3 \times 1200.0$ & 2.9 & 1.49 & Photo \\
\hline U 772 & $B$ & 0.190 & 22 & $6 \times 510.0$ & 2.0 & 1.18 & Photo \\
\hline U1171 & $B$ & Oct 90 & 22 & $6 \times 420.0$ & 1.9 & 1.10 & Photo \\
\hline \multirow[t]{4}{*}{ K10 } & $B$ & Oct 90 & 22 & $3 \times 600.0$ & 2.7 & 1.15 & Photo \\
\hline & If & Oct 90 & 22 & $3 \times 120.0$ & 1.0 & 1.07 & F'hoto \\
\hline & $B$ & Jan93 & 23 & $1 \times 600.0$ & 1.2 & 1.16 & Cirrus \\
\hline & 1 & Jan93 & 23 & $3 \times 420.0$ & 1.2 & 1.23 & Oirrus \\
\hline \multirow[t]{4}{*}{ U1981 } & $B$ & $O \cdot 190$ & 21 & $3 \times 600.0$ & 1.4 & 1.34 & Photo \\
\hline & 15 & $O \cdot 190$ & 21 & $2 \times 420.0$ & 1.0 & 1.24 & Photo \\
\hline & $B$ & Jan93 & 24 & $1 \times 600.0$ & 1.8 & 1.90 & Cirrus \\
\hline & $I$ & $\operatorname{Jan} 93$ & 24 & $3 \times 420.0$ & 1.5 & 1.74 & Cirrus \\
\hline \multirow[t]{4}{*}{$\mathrm{U} 2017$} & $B$ & $\operatorname{Oct} 90$ & 21 & $3 \times 600.0$ & 1.1 & 1.05 & Fhoto \\
\hline & If & Oct 90 & 21 & $3 \times 120.0$ & 1.0 & 1.11 & F'hoto \\
\hline & $B$ & Jan93 & 24 & $3 \times 600.0$ & 1.5 & 1.05 & Oirrus \\
\hline & 1 & Jang3 & 24 & $3 \times 420.0$ & 1.2 & 1.02 & Oirrus \\
\hline \multirow[t]{4}{*}{ U.2034 } & $B$ & $O \cdot 190$ & 22 & $1 \times 180.0$ & 1.4 & 1.29 & Photo \\
\hline & $I f$ & Oct 90 & 22 & $9 \times 180.0$ & 0.7 & 1.30 & Photo \\
\hline & $B$ & $\operatorname{Jan} 93$ & 23 & $3 \times 600.0$ & 1.7 & 1.13 & Cirrus \\
\hline & I & Jan93 & 23 & $3 \times 120.0$ & 1.2 & 1.19 & Cirmus \\
\hline \multirow[t]{2}{*}{$\mathrm{U} 20, \overline{3} 3$} & $B$ & Oct 90 & 20 & $3 \times 600.0$ & 2.2 & 1.17 & Fhoto \\
\hline & 1 & 0.190 & 20 & $2 \times 1200.0$ & 1.9 & 1.08 & Photo \\
\hline \multirow[t]{4}{*}{ U 2162} & $B$ & 0.190 & 22 & $3 \times 600.0$ & 1.2 & 1.27 & Photo \\
\hline & $I f$ & Oct 90 & 22 & $3 \times 420.0$ & 0.9 & 1.35 & Photo \\
\hline & $B$ & $\operatorname{Jan} 93$ & 24 & $3 \times 600.0$ & 1.5 & 1.33 & Cirrus \\
\hline & $I$ & $\operatorname{Jan} 93$ & 24 & $3 \times 420.0$ & 1.2 & 1.44 & Cirrus \\
\hline \multirow[t]{4}{*}{$\mathrm{K} 37$} & $B$ & Oct90 & 21 & $3 \times 600.0$ & 1.1 & 1.28 & Fhoto \\
\hline & $T$ & Oct 90 & 21 & $3 \times 600.0$ & 1.0 & 1.25 & Fhoto \\
\hline & $B$ & Sep91 & 16 & $1 \times 300.0$ & 1.8 & 1.24 & Photo \\
\hline & 15 & Sep91 & 15 & $2 \times 450.0$ & 2.4 & 1.25 & Photo \\
\hline \multirow[t]{2}{*}{$\mathrm{K} 37 \mathrm{C}$} & $B$ & Scp91 & 16 & $2 \times 300.0$ & 1.8 & 1.25 & Photo \\
\hline & $I$ & Scpo1 & 15 & $1 \times 600.0$ & 2.4 & 1.25 & Photo \\
\hline \multirow[t]{2}{*}{ U:3212 } & $B$ & Oct90 & 21 & $3 \times 600.0$ & 1.2 & 1.29 & Photo \\
\hline & $I f$ & Oct 90 & 21 & $3 \times 120.0$ & 1.2 & 1.30 & Fhoto \\
\hline \multirow[t]{2}{*}{ U33881 } & $B$ & Apr91 & 17 & $3 \times 600.0$ & 1.7 & 1.69 & Fhoto \\
\hline & 15 & $\Lambda_{p r 91}$ & 17 & $3 \times 420.0$ & 1.4 & 1.61 & Photo \\
\hline \multirow[t]{2}{*}{03817} & $B$ & 0.190 & 21 & $3 \times 600.0$ & 1.4 & 1.04 & Photo \\
\hline & $I f$ & Oct90 & 21 & $3 \times 420.0$ & 1.0 & 1.03 & Photo \\
\hline U:3860 & $B$ & Oct 90 & 22 & $3 \times 583.0$ & 1.4 & 1.04 & Photo \\
\hline \multirow[t]{2}{*}{ U3966 } & $B$ & Apr 91 & 18 & $3 \times 600.0$ & 1.7 & 1.23 & Cirrus \\
\hline & $T$ & Apr91 & 18 & $3 \times 120.0$ & 1.1 & 1.11 & Cirmus \\
\hline [U.1204 & $B$ & Apr91 & 19 & $3 \times 600.0$ & 2.0 & 1.21 & Cirrus \\
\hline & 1 & $\Lambda_{p r 91}$ & 19 & $2 \times 420.0$ & 1.4 & 1.17 & Oirrus \\
\hline (] 4173 & $B$ & $\Lambda p r 91$ & 18 & $3 \times 600.0$ & 2.7 & 1.65 & Oirrus \\
\hline & $I$ & Apr91 & 18 & $3 \times 420.0$ & 2.7 & 1.61 & Cirrus \\
\hline & B & $\operatorname{Jan} 93$ & 23 & $2 \times 600.0$ & 1.2 & 1.51 & Photo \\
\hline & $T$ & Jan93 & 23 & $2 \times 120.0$ & 1.2 & 1.50 & Fhoto \\
\hline MS1dwA & $B$ & Apr9l & 17 & $3 \times 600.0$ & 1.7 & 1.52 & Fhoto \\
\hline & $T$ & Apr91 & 17 & $3 \times 120.0$ & 1.1 & 1.16 & Fhoto \\
\hline & $B$ & Jang3 & 23 & $3 \times 600.0$ & 1.2 & 1.34 & Photo \\
\hline & 1 & Jan93 & 23 & $3 \times 420.0$ & 1.4 & 1.37 & Photo \\
\hline$M 81 \mathrm{dw} B$ & $B$ & $\operatorname{Jan} 93$ & 24 & $3 \times 600.0$ & 1.8 & 1.34 & Cirrus \\
\hline & $I$ & $\operatorname{Jan} 93$ & 24 & $3 \times 420.0$ & 1.5 & 1.30 & Cirrus \\
\hline บั. 706 & $B$ & Apr91 & 21 & $3 \times 600.0$ & 17.1 & 00 & Fhoto \\
\hline & $T$ & Apr9l & 21 & $3 \times 120.0$ & 1.1 & 1.00 & Fhoto \\
\hline
\end{tabular}


Table 3 continucd

\begin{tabular}{|c|c|c|c|c|c|c|c|}
\hline Galaxy & band & run 1 & night & $\begin{array}{l}\text { cxp. timc } \\
\text { (\#xsec) }\end{array}$ & $\begin{array}{l}\text { sccing } \\
\text { arcsec. }\end{array}$ & airmass & $\begin{array}{c}\text { sky } \\
\text { conditions }\end{array}$ \\
\hline \multirow[t]{2}{*}{ U5709 } & $B$ & Apr91 & 17 & $3 \times 600.0$ & 3.4 & 1.36 & Photo \\
\hline & $I$ & Apr 91 & 17 & $4 \times 46.5 .0$ & 1.4 & 1.21 & Photo \\
\hline \multirow[t]{2}{*}{ U6 151} & $B$ & Apr91 & 20 & $3 \times 600.0$ & 2.0 & 1.05 & Photo \\
\hline & $I$ & Apr.91 & 20 & $3 \times 120.0$ & 1.7 & 1.03 & Photo \\
\hline \multirow[t]{2}{*}{ U6248 } & $B$ & $\Lambda_{\mathrm{pr}} 91$ & 21 & $3 \times 600.0$ & 1.7 & 1.09 & Photo \\
\hline & 1 & $\Lambda_{p r} 91$ & 21 & $3 \times 420.0$ & 1.4 & 1.08 & Photo \\
\hline \multirow[t]{2}{*}{$\mathrm{U} 6596$} & $B$ & Apr91 & 19 & $3 \times 600.0$ & 1.4 & 1.10 & Cirrus \\
\hline & $I$ & Apr 91 & 19 & $3 \times 420.0$ & 1.4 & 1.10 & Cirrus \\
\hline \multirow[t]{3}{*}{ U6628 } & $B$ & Apr91 & 17 & $3 \times 300.0$ & 2.4 & 1.35 & Photo \\
\hline & $I$ & Apr:91 & 17 & $3 \times 300.0$ & 2.0 & 1.26 & Fhoto \\
\hline & $B$ & Apr93 & 20 & $2 \times 600.0$ & 1.7 & 1.06 & Cirmus \\
\hline \multirow[t]{2}{*}{ U7295 } & $B$ & $\Lambda_{p r} 91$ & 20 & $3 \times 600.0$ & 3.1 & 1.01 & Photo \\
\hline & 1 & $\Lambda_{p r} 91$ & 20 & $3 \times 240.0$ & 1.7 & 1.00 & Photo \\
\hline \multirow[t]{2}{*}{ U7548 } & $B$ & Apr91 & 19 & $3 \times 600.0$ & 2.0 & 1.10 & Cirrus \\
\hline & $I$ & Apr91 & 19 & $3 \times 420.0$ & 2.0 & 1.07 & Cirrus \\
\hline \multirow{2}{*}{ U7596 } & $B$ & Apr91 & 19 & $3 \times 600.0$ & 1.4 & 1.36 & Photo \\
\hline & $I$ & Apr91 & 19 & $3 \times 120.0$ & 1.7 & 1.26 & Fhoto \\
\hline \multirow{3}{*}{$\llbracket 7608$} & $B$ & Apr91 & 18 & $3 \times 600.0$ & 1.1 & 1.04 & Girmus \\
\hline & 1 & $\Lambda_{\mathrm{pr}} 91$ & 18 & $3 \times 420.0$ & 1.2 & 1.03 & Girrus \\
\hline & $B$ & $\Lambda_{p r} 93$ & 18 & $3 \times 600.0$ & 1.7 & 1.03 & Oirrus \\
\hline \multirow[t]{2}{*}{$\mathrm{U} 7636$} & $B$ & Apr 91 & 21 & $3 \times 600.0$ & 1.4 & 1.11 & Photo \\
\hline & $I$ & Apr91 & 21 & $3 \times 420.0$ & 1.4 & 1.10 & Photo \\
\hline \multirow[t]{2}{*}{$\mathrm{U} 7684$} & $B$ & Apr 91 & 21 & $3 \times 600.0$ & 1.7 & 1.13 & Photo \\
\hline & $J$ & Apr:91 & 21 & $3 \times 120.0$ & 1.1 & 1.08 & Fhoto \\
\hline \multirow[t]{2}{*}{$\mathrm{U} 78882$} & $B$ & Apr91 & 19 & $3 \times 600.0$ & 2.0 & 1.00 & Cirmus \\
\hline & 1 & $\Lambda_{p r 91}$ & 19 & $3 \times 420.0$ & 1.5 & 1.01 & Oirrus \\
\hline \multirow[t]{2}{*}{ U8091 } & $B$ & $\Lambda_{\mathrm{pr}} 91$ & 21 & $2 \times 600.0$ & 1.4 & 1.34 & Cirrus \\
\hline & $I$ & Apr 91 & 21 & $2 \times 420.0$ & 1.4 & 1.23 & Cirrus \\
\hline U8201 & $B$ & Apr93 & 19 & $2 \times 600.0$ & 2.0 & 1.32 & Cirrus \\
\hline \multirow[t]{2}{*}{ IT 86883} & $B$ & Apr91 & 21 & $3 \times 600.0$ & 1.1 & 1.29 & Photo \\
\hline & $J$ & Aprol & 21 & $3 \times 120.0$ & 1.1 & 1.20 & Fhoto \\
\hline$\lfloor 8760$ & $B$ & Aprol & 19 & $3 \times 600.0$ & 1.1 & 1.23 & Cirmus \\
\hline & 1 & $\Lambda_{p r} 91$ & 19 & $4 \times 465.0$ & 1.5 & 1.14 & Oirrus \\
\hline U8833 & $B$ & $\Lambda_{\mathrm{pr}} 91$ & 19 & $4 \times 570.0$ & 1.7 & 1.58 & Photo \\
\hline & $I$ & Apr 91 & 19 & $6 \times 390.0$ & 1.0 & 1.48 & Photo \\
\hline U9128 & $B$ & Apr91 & 21 & $2 \times 600.0$ & 1.9 & 1.56 & Photo \\
\hline & $I$ & Apr91 & 21 & $3 \times 120.0$ & 1.5 & 1.42 & Fhoto \\
\hline U.9391 & $B$ & Aprol & 17 & $3 \times 600.0$ & 2.0 & 1.21 & Cirmus \\
\hline & $J$ & Apr91 & 17 & $3 \times 120.0$ & 2.2 & 1.18 & Cirmus \\
\hline U10031 & $B$ & May 85 & 23 & $1 \times 600.0$ & 2.4 & 1.44 & Photo \\
\hline & $R$ & May 85 & 23 & $1 \times 600.0$ & 2.1 & 1.41 & Photo \\
\hline & $I$ & Apr 91 & 20 & $3 \times 420.0$ & 1.4 & 1.18 & Photo \\
\hline U10058 & $B$ & May 8.5 & 23 & $1 \times 600.0$ & 1.8 & 1.28 & Photo \\
\hline & R & Mays: & 23 & $1 \times 600.0$ & 1.8 & 1.33 & Fhoto \\
\hline & $J$ & Apr91 & 17 & $3 \times 120.0$ & 1.7 & 1.08 & Cirmus \\
\hline U10290 & $B$ & May8: & 22 & $1 \times 900.0$ & 1.8 & 1.39 & Fhoto \\
\hline & $R$ & May 85 & 22 & $2 \times 300.0$ & 1.8 & 1.34 & Photo \\
\hline & 1 & $\Lambda_{p r 91}$ & 17 & $3 \times 300.0$ & 1.7 & 1.29 & Oirrus \\
\hline $\mathrm{U} 10376$ & $B$ & May 8.5 & 23 & $1 \times 900.0$ & 1.5 & 1.27 & Photo \\
\hline & $R$ & May 8.5 & 23 & $2 \times 450.0$ & 1.5 & 1.25 & Photo \\
\hline & $I$ & Apr91 & 20 & $3 \times 120.0$ & 1.1 & 1.22 & Fhoto \\
\hline U10669 & $B$ & May8: & 22 & $1 \times 900.0$ & 1.8 & 1.33 & Fhoto \\
\hline & R & May 85 & 22 & $2 \times 300.0$ & 1.5 & 1.35 & Photo \\
\hline & 1 & $\Lambda_{\mathrm{pr} 91}$ & 20 & $3 \times 420.0$ & 1.4 & 1.30 & Photo \\
\hline U11764 & $B$ & May 85 & 22 & $1 \times 900.0$ & 2.1 & 1.21 & Photo \\
\hline & $R$ & May8.5 & 22 & $1 \times 600.0$ & 1.8 & 1.26 & Photo \\
\hline U12082 & $B$ & Scp91 & 13 & $3 \times 300.0$ & 1.8 & 1.27 & Photo \\
\hline & $I$ & Sep91 & 13 & $2 \times 300.0$ & 1.8 & 1.04 & Fhoto \\
\hline 012151 & $B$ & May8: & 23 & $1 \times 600.0$ & 1.5 & 1.61 & F'hoto \\
\hline & $R$ & May85 & 23 & $1 \times 600.0$ & 1.5 & 1.53 & Photo \\
\hline U12894 & $B$ & 0.190 & 21 & $3 \times 600.0$ & 1.4 & 1.14 & Photo \\
\hline & $1 \mathrm{~J}$ & $0: 190$ & 21 & $3 \times 420.0$ & 1.4 & 1.09 & Photo \\
\hline
\end{tabular}


$B\left(\lambda_{0}=1320 \AA\right.$, $\left.F \mathrm{WH}=1061 \AA\right)$ and Mould / filters were used for the remaining runs (Sep91 and Jan93). The different filters had the effect of changing the transformation coefficients for the different runs (see 82.3 ). The transmission curves for the Harris and Mould filters are shown in Figure 1.

The photometry was calibrated by making repeated observations of several standard stars from Landolt (1983) throughout the night. Fainter standards from the new list of Landolt (1992) were used during the $\Lambda$ pr 91 and Jan 93 runs. During the May 85 run, additional observations were made of a standard field in Selected Area 57 which contained lainter stars, with a moderate range in color, and photometry provided by Sandage (1985). Obscrvations of the globular clusters identified by Christian et al. (1985), with photometry from l)avis (1991), were made during the Apr 91, Jan 93, and Apr 93 runs. In addition, M67 (Schild, 1983) was observed during the Apr 93 run.

Dome flats using the standard color corrected lamps and a white reflective spot on the inside of the dome were taken at the beginning and end of each night. A sequence of $\sim 20$ bias frames were also taken at this time. In addition, twilight flats of the sky were obtained each night in each filter, usually in the evening. $\Lambda$ "blank" field, containing lew bright stars, from Christian $t$ l al. (1985) was imaged several times with slight oflsets between exposures during each run (except for May 85 and Jan 93). Since the May 85 and Jan 93 runs were made up mostly of observations of point sources (Thuan et al. 1992, and Gelderman 1991), these object frames were combined to form equivalent blank dark sky and fringe frames (sce following section). 


\subsection{Data Reduction}

The CCD data were reduced by using the IR $\Lambda \mathrm{F}^{2}$ solt ware package CCDRED. The following description applies in particular to the TEK2/T5HA $512 \times 512$ chip which was used to obtain the majority of the data, but all the data were reduced using the same basic procedure (sce Masscy \& Jacoby 1992).

First, a bias oflset, equal to the mean of 32 columns in the overscan region from cach row, was subtracted from cach frame. 'The resulting images were then trimmed to a $508 \times 508$ format. A composite zero frame, the average of 20 individual bias frames (with the overscan already subtracted), was constructed with the ZEROCOMBINE task and then subtracted from all frames to compensate for pixel-to-pixel variations in the bias level. This bias pattern was found to be very stable throughout the night and from night to night.

High signal-to-noise level dome flats for each night were combined with FLATCOMBINE, which scales the individual flats by the median to create a master dome flat, for the removal of the pixel to pixel variation in the detector semsitivity. The twilight sky flats and/or the blank dark sky flats where then combined with the dome flats using MKILLUMCOR, which heavily smooths the lower signal to noise sky flats so that they can be used to remove the large scale variation of the detector. The dome flats may not illuminate the (CI) in the same way as the night sky does

\footnotetext{
${ }^{2} \mathrm{IR} \Lambda \mathrm{F}$ is distributed by National (Optical Astronomy (Observatories, which is operated by the Association of Universities for Research in Astronomy, Inc., under cooperative agreement with the National Science Foundation
} 
because of light leaks, uneven illumination of the white spot on the dome, or incorrect color balance of the lamps. As a result, the large scale variation of the detector can only be removed with sky lats, preferably blank dark sky lats, to achieve the best color match with the object frames.

The blank dark sky exposures in the $I$ - and $R$-bands were used to remove the interference fringe patterm produced by night sky emission lines. In this case the blank dark sky frames were not smoothed as described above. Instead they were processed 11sing CCDRED in which the overscan is removed, the master zero frame is subtracted, the image is trimmed, and finally the master flat for the night is scaled and divided into the blank dark sky exposure. The individual frames from the same night and in the same filter are combined using COMBINE. The median value of the resultant frame is determined and subtracted from the frame, leaving a fringe frame with a median of zero. This fringe frame is then scaled by factors of 0.1 to 2.0 and subtracted from the fringed galaxy images which had previously been processed through the flat fielding stage.

At this point, the innges were inspected to see which subtraction had produced the most complete removal of the fringes. In this way, the fringes were removed without changing the average value across the frame. 'This trial and croo process is necessary because the fringe levels change throughout the night depending on the relative position of the sun with respect to the upper atmosphere where the night sky emission lines are produced.

The blank dark sky exposures for the Oct 90 run were obtained on the first clear night of the run, after which the secondary of the $2.1 \mathrm{~m}$ telescope shifted notice- 
ably (throwing the focus off by a very large amount, among other things). It was later discovered that the optical path had changed enough to render the resulting frames useless for removing fringes from data taken after the secondary shift. The frames were still found to be sufficient for the removal of large scale variations as flat field frames. Therefore, the $I$-band frames of eleven galaxies were contaminated by fringes: six of these objects were subsequently reobserved leaving just five galaxics with some fringes. These objects are indicated by an $f$ next to the $/$ in the band column of Table 3 . The fringes cause a $\sim 2 \%$ systematic variation on a fairly small $(\sim 20$ pixel) spatial scale.

After the individual object frames were processed through the flat fielding stage using CCDRED (and fringes removed as described above from the $I$ - and $R$-band frames), the separate exposures were aligned using IMALIGN. This routine determines the centroids of the stars in the frame and then shilts and trims the images. The COMBINE task was then used to combine the images by filter, using average sigma clipping to remove most of the cosmic rays and bad pixels. Of course, in the few cases in which there were only two images taken in a filter, the images could only be averaged (which will not remove the cosmic rays). 'I'he COSMICRAYS task was then used to remove nearly all of the remaining radiation events in the combined frames, after which IMEDIT was used to remove any remaining blemishes. At this point, the systematic variation across the frame was $\sim 2 \%$. 


\subsubsection{Sky Subtraction}

A low order polynomial, usually a tilted plane, was fit to the sky surrounding the galaxy on cach frame, using IMSURFIT. Great care was taken to avoid including in the fit any of the outer regions of the galaxy itself or the halos of bright stars. The sky subtraction proved to be particularly critical because of the large angular size and low surface brightness of many of these nearby galaxies and the relatively small field of view of most of the observalions.

It was found empirically that the shape of the sky could be best fit by setting sigma rejection to 3 sigma for both the lower and upper rejection $\left(3 \sigma_{\mathrm{T}}, 3 \sigma_{\mathrm{U}}\right)$, while the median value for the sky intensity was best determined with 3 sigma lower rejection and 0 sigma upper rejection $\left(3 \sigma_{\mathrm{L}}, 0 \sigma_{\mathrm{L}}\right)$. 'This was possibly due to the presenec of numerous background stars which contribute only in a positive sense to the sky intensity. Therefore the $\left(3 \sigma_{\mathrm{L}}, 3 \sigma_{\mathrm{U}}\right)$ frame was sealed to the level of the $\left(3 \sigma_{\mathrm{L}}, 0 \sigma_{U}\right)$ frame. The scaling of the sky frame in this way did not introduce much additional error since the difference between the medians of the two frames was less than $\sim 1 \%$. which is the approximate error in the sky level determination itself. This resulting sky frame was then subtracted from the image, and this sky-subtracted frame was then divided by the sky frame to produce a normalized sky-subtracted frame. This technique of normalizing the object frames serves to remove most of the residual local crrors (Pence 1978; Carignan 1983 and 1985; Ichikawa, Wakamatsu, \& Okamura 1986). 'l'he pixel to pixel variation across the frame at the end of the reduction was less than $\sim 1 \%$. 
The effective exposure time, or the mean of the exposure times for all of the images that were combined, the effective airmass determined with the SETAIRMASS task, and the sky level in $\Lambda \mathrm{DU}$ obtained from the median of the $\left(3 \sigma_{\mathrm{I}}, 0 \sigma_{\mathrm{U}}\right)$ frame, were then added to the header using HEDIT. Finally the images were reoriented so that Torth was at the top and East at the lelt using IMTRANSPOSE and/or ROTATE, depending on the original orientation.

\subsection{Photometric Calibration}

Aperture photometry was performed on the standard star observations using the PHOT task in IRAF. An aperture, 11sually 30 pixols in radius (at lcast $5 \times \mathrm{F} W \mathrm{HM}$ ), was automatically centered on the standard to be measured, then a radial profile was extracted and examined to determine whether the standard was saturated or contaminated by fainter stars. The most frequently occurring valıe, or mode, of the pixel values in a sky annulus was determined (after sigma rejection) and this sky level was subtracted. The resulting counts within the aperture were summed up and converted to a PHOT instrumental magnitude using

$$
\mathrm{ml}_{\text {phot }}=-2.5 \log \left[\sum^{a \text { porture }} \frac{\mathrm{ADU}}{t}\right]+\text { zmag. }
$$

where ADU is the number of counts in Analog to Digital Units, $t$, is the exposure time in seconds and zmag is an arbitrary, but fixed, zero point selected so that the magnitudes come out to reasonable numbers. In this work, zmag was always set equal to 26. 
For crowded fields typical of the globular cluster fields of Davis (1991), it is necessary to use a much smaller aperture to avoid contamination from neighboring stars. In these cases, several bright and relatively isolated stars in the frame are selected and measured using both this smaller aperture and the larger ( 30 pixel) aperture, while the remaining stars are measured using only the smaller aperture (typically $\sim 5$ pixels). Again, the radial profiles are uscful for detecting faint nearby stars. Aperture corrections are then determined for each frame by averaging the corrections for each of the bright, uncontaminated stars selected.

The PHOTCAL package was then 1used to determine the transformation equations for photometric calibration. Transformation equations of the form

$$
\begin{gathered}
B-I=\alpha_{(B-I)}\left[\left(b_{p h o t}-k_{B} * X_{B}\right)-\left(i_{p h o t}-k_{I} * X_{I}\right)\right]+\zeta_{(B-I)} \\
B=\alpha_{B}[B-I]+\left(b_{p h o l}-k_{B} * X_{B}\right)+\zeta_{B} \\
I=\alpha_{I}[B-I]+\left(i_{p h o l}-k_{I} * X_{I}\right)+\zeta_{I}
\end{gathered}
$$

were set up and verified with the MKCONFIG and CHKCONFIG lasks. IIere $\alpha$ is the slope and $\zeta$ the zero point of the color and magnitude equations, $k$ is the extinction coefficient and $X$ the airmass, and $b_{p h o t}$ and $i_{\text {phot }}$ are the instrumental $B$ and / magnitudes from equation 2.1 .

I'he parameter fitting procedure requires that certain information be prepared for input into the PHOTCAL fitting routine, FITPARAMS, using the routines MKIMSETS and MKNOBSFILE. These include the instrumental magnitude of the standards, the image header files for each observation, the aperture corrections for each image, and a catalog of standard star magnitudes and colors. 
Table 4 a

$2.1 \mathrm{~m}$ Photometric Calibration

\begin{tabular}{|c|c|c|c|c|c|c|c|c|c|c|}
\hline \multirow[b]{2}{*}{$\begin{array}{l}\text { Run } \\
\text { Night }\end{array}$} & \multirow[b]{2}{*}{$\begin{array}{l}\# \text { ol } \\
\text { stds }\end{array}$} & \multicolumn{3}{|c|}{ Extinclion Goellicients } & \multicolumn{3}{|c|}{ liansformalion Slopes } & \multicolumn{3}{|c|}{ Iräansformation Zero Points } \\
\hline & & $\begin{array}{l}\mathrm{k}_{\mathrm{B}} \\
(\sigma)\end{array}$ & $\begin{array}{l}\mathrm{k}_{1} \\
(\sigma)\end{array}$ & $\begin{array}{c}\mathrm{k}_{\mathrm{B}-1} \\
(\sigma)\end{array}$ & $\begin{array}{l}a_{B} \\
(\sigma)\end{array}$ & $\begin{array}{l}<x_{1} \\
(\sigma)\end{array}$ & $\begin{array}{c}\alpha_{B-1} \\
(\sigma)\end{array}$ & $\begin{array}{c}\zeta_{\mathrm{B}} \\
(\sigma) \\
{[\mathrm{RMS}]}\end{array}$ & $\begin{array}{c}\zeta_{1} \\
(\sigma) \\
{[\mathrm{RMS}]}\end{array}$ & $\begin{array}{c}\zeta_{\mathrm{B}-1} \\
(\sigma) \\
{[\mathrm{RMS}]}\end{array}$ \\
\hline $\begin{array}{r}\text { Oct } \\
1990\end{array}$ & 26 & $\begin{array}{c}0.1619 \\
(0.0369)\end{array}$ & $\begin{array}{c}0.0576 \\
(0.0183)\end{array}$ & $\begin{array}{c}0.1120 \\
(0.0375)\end{array}$ & $\begin{array}{c}0.0811 \\
(0.0037)\end{array}$ & $\begin{array}{c}0.0047 \\
(0.0016)\end{array}$ & $\begin{array}{c}1.0781 \\
(0.0037)\end{array}$ & $\ldots$ & $\cdots \cdots \cdots$ & $\cdots$ \\
\hline 20 & 10 & $\ldots \ldots \ldots$ & $\ldots \ldots \ldots$ & $\ldots \ldots \ldots$ & $\ldots \ldots$ & $\ldots \ldots \ldots$ & $\ldots \ldots \ldots$ & $\begin{array}{c}-2.0504 \\
(0.0049) \\
{[0.085]}\end{array}$ & $\begin{array}{c}-2.8864 \\
(0.0031) \\
{[0.027]}\end{array}$ & $\begin{array}{c}0.9074 \\
(0.0043) \\
{[0.062]}\end{array}$ \\
\hline 21 & 9 & $\ldots \ldots$ & $\ldots \ldots \ldots$ & $\ldots$. & $\ldots \ldots$ & $\ldots \ldots \ldots$ & $\ldots \ldots \ldots$ & $\begin{array}{c}-2.0250 \\
(0.0043) \\
{[0.081]}\end{array}$ & $\begin{array}{c}-2.8798 \\
(0.0023) \\
{[0.026]}\end{array}$ & $\begin{array}{c}0.9252 \\
(0.0045) \\
{[0.060]}\end{array}$ \\
\hline 22 & $i$ & $\ldots \ldots$ & $\ldots \ldots \ldots$ & $\ldots \ldots \ldots$ & $\ldots \ldots \ldots$ & $\ldots \ldots$ & $\ldots \ldots \ldots$ & $\begin{array}{c}-2.0215 \\
(0.0039) \\
{[0.091]}\end{array}$ & $\begin{array}{c}-2.8957 \\
(0.0064) \\
{[0.051]}\end{array}$ & $\begin{array}{c}0.9495 \\
(0.0164) \\
{[0.063]}\end{array}$ \\
\hline $\begin{array}{r}\Lambda p r \\
1991\end{array}$ & 64 & $\begin{array}{c}0.1935 \\
(0.0393)\end{array}$ & $\begin{array}{c}0.0412 \\
(0.0205)\end{array}$ & $\begin{array}{c}0.1699 \\
(0.0154)\end{array}$ & $\begin{array}{c}0.0711 \\
(0.0043)\end{array}$ & $\begin{array}{l}-0.0103 \\
(0.0039)\end{array}$ & $\begin{array}{c}1.0926 \\
(0.0041)\end{array}$ & $\ldots \ldots \ldots$ & $\ldots \ldots \ldots$ & $\ldots \ldots \ldots$ \\
\hline 17 & 28 & $\ldots \ldots \ldots$ & $\ldots \ldots \ldots$ & $\ldots \ldots \ldots$ & $\ldots \ldots \ldots$ & $\ldots \ldots \ldots$ & $\ldots \ldots$ & $\begin{array}{c}-2.0254 \\
(0.0066) \\
{[0.116]}\end{array}$ & $\begin{array}{c}-2.9208 \\
(0.0073) \\
{[0.076]}\end{array}$ & $\begin{array}{c}0.9741 \\
(0.0019) \\
{[0.195]}\end{array}$ \\
\hline 18 & 59 & $\ldots \ldots$ & ....... & n..... & $\ldots \ldots$ & $\ldots \ldots \ldots$ & n..... & $\begin{array}{c}-2.0795 \\
(0.0218) \\
{[1.828]}\end{array}$ & $\begin{array}{c}-2.9901 \\
(0.0092) \\
{[0.917]}\end{array}$ & $\begin{array}{c}0.8450 \\
(0.0726) \\
{[0.962]}\end{array}$ \\
\hline 19 & 17 & $\ldots \ldots \ldots$ & $\ldots \ldots \ldots$ & $\ldots \ldots$ & $\ldots \ldots$ & ........ & $\ldots \ldots \ldots$ & $\begin{array}{c}-2.0794 \\
(0.0073) \\
{[0.461]}\end{array}$ & $\begin{array}{c}-2.9261 \\
(0.0039) \\
{[0.049]}\end{array}$ & $\begin{array}{c}0.9536 \\
(0.0102) \\
{[0.554]}\end{array}$ \\
\hline 20 & 50 & $\ldots \ldots$ & $\ldots \ldots \ldots$ & $\ldots \ldots \ldots$ & $\ldots \ldots \ldots$ & $\ldots \ldots$ & $\ldots \ldots \ldots$ & $\begin{array}{c}-2.0315 \\
(0.0034) \\
{[0.331]}\end{array}$ & $\begin{array}{c}-2.897 t \\
(0.0033) \\
{[0.011]}\end{array}$ & $\begin{array}{c}0.9431 \\
(0.0079) \\
{[0.377]}\end{array}$ \\
\hline 21 & 31 & $\ldots \ldots$ & $\ldots \ldots \ldots$ & $\ldots \ldots$ & $\ldots \ldots$ & $\ldots \ldots$ & $\ldots \ldots \ldots$ & $\begin{array}{c}-2.0221 \\
(0.0042) \\
{[0.035]}\end{array}$ & $\begin{array}{c}-2.8981 \\
(0.0025) \\
{[0.038]}\end{array}$ & $\begin{array}{c}0.9627 \\
(0.0047) \\
{[0.065]}\end{array}$ \\
\hline $\begin{array}{r}\text { Jan } \\
1993\end{array}$ & 118 & $\begin{array}{c}0.3079 \\
(0.0282)\end{array}$ & $\begin{array}{c}0.0999 \\
(0.0289)\end{array}$ & $\begin{array}{c}0.2887 \\
(0.0307)\end{array}$ & $\begin{array}{c}0.0 .509 \\
(0.00 ; 1)\end{array}$ & $\begin{array}{c}0.0023 \\
(0.0075)\end{array}$ & $\begin{array}{c}1.0160 \\
(0.0071)\end{array}$ & (....... & ........ & ….... \\
\hline 23 & 50 & $\ldots \ldots$ & $\ldots \ldots \ldots$ & $\ldots \ldots \ldots$ & $\ldots \ldots$ & $\ldots \ldots$ & $\ldots \ldots$ & $\begin{array}{c}-1.7061 \\
(0.0052) \\
{[0.060]}\end{array}$ & $\begin{array}{c}-2.2658 \\
(0.0085) \\
{[0.113]}\end{array}$ & $\begin{array}{c}0.6227 \\
(0.0103) \\
{[0.098]}\end{array}$ \\
\hline 21 & 68 & $\ldots \ldots$ & ........ & ........ & ........ & ........ & ........ & $\begin{array}{c}-1.7762 \\
(0.0067) \\
{[0.053]}\end{array}$ & $\begin{array}{c}-2.3066 \\
(0.0070) \\
{[0.076]}\end{array}$ & $\begin{array}{c}0.5789 \\
(0.0071) \\
{[0.058]}\end{array}$ \\
\hline
\end{tabular}


Table 4b

0.9m Photometric Calibration

\begin{tabular}{|c|c|c|c|c|c|c|c|c|c|c|}
\hline \multirow[b]{2}{*}{$\begin{array}{l}\text { Run } \\
\text { Night }\end{array}$} & \multirow[b]{2}{*}{$\begin{array}{l}\text { \# of } \\
\text { slds }\end{array}$} & \multicolumn{3}{|c|}{ Fixtinction Coefficients } & \multicolumn{3}{|c|}{ Transformation Slopes } & \multicolumn{3}{|c|}{ Transformation Zero Points } \\
\hline & & $\begin{array}{l}k_{\mathrm{B}} \\
(\sigma)\end{array}$ & $\begin{array}{l}k_{\mathrm{T}} \\
(\sigma)\end{array}$ & $\begin{array}{c}k_{R-T} \\
(\sigma)\end{array}$ & $\begin{array}{l}\alpha_{\mathrm{B}} \\
(\sigma)\end{array}$ & $\begin{array}{l}\alpha_{\mathbf{T}} \\
(\sigma)\end{array}$ & $\begin{array}{c}x_{B}-\mathrm{T} \\
(\sigma)\end{array}$ & $\begin{array}{c}S \mathrm{~B} \\
(\sigma) \\
{[\mathrm{HNS}]}\end{array}$ & $\begin{array}{c}\zeta_{T} \\
(\sigma) \\
{[\mathrm{K}, \mathrm{S}]}\end{array}$ & $\begin{array}{c}(\mathrm{B}-\mathrm{T} \\
(\sigma) \\
{[\text { HMS }]}\end{array}$ \\
\hline $\begin{array}{l}\text { Scp } \\
1991\end{array}$ & 66 & $\begin{array}{c}0.3359 \\
(0.0276)\end{array}$ & $\begin{array}{c}0.088 .5 \\
(0.0439)\end{array}$ & $\begin{array}{c}0.2183 \\
(0.05787)\end{array}$ & $\begin{array}{c}0.0321 \\
(0.0051)\end{array}$ & $\begin{array}{c}-0.00799 \\
(0.0013)\end{array}$ & $\begin{array}{c}1.0572 \\
(0.0061)\end{array}$ & $\ldots \ldots \ldots$ & $\ldots \ldots \ldots$ & $\cdots \cdots \cdots$ \\
\hline 13 & 12 & $\ldots \ldots$ & $\ldots \ldots$ & $\ldots \ldots \ldots$ & $\ldots \ldots$ & $\ldots \ldots \ldots$ & $\ldots \ldots$ & $\begin{array}{c}-1.0851 \\
(0.0075) \\
{[0.024]}\end{array}$ & $\begin{array}{c}-1.6846 \\
(0.0103) \\
{[0.025]}\end{array}$ & $\begin{array}{c}0.56399 \\
(0.0180) \\
{[0.044]}\end{array}$ \\
\hline 14 & 11 & $\ldots \ldots$ & $\ldots \ldots$ & $\ldots \ldots \ldots$ & $\ldots \ldots$ & $\ldots \ldots \ldots$ & $\ldots \ldots$ & $\begin{array}{c}-4.0371 \\
(0.0 \mid 03) \\
{[0.033]}\end{array}$ & $\begin{array}{c}-4.6662 \\
(0.0061) \\
{[0.016]}\end{array}$ & $\begin{array}{c}0.5965 \\
(0.0092) \\
{[0.021]}\end{array}$ \\
\hline 15 & 17 & $\ldots \ldots$ & $\ldots \ldots$ & $\ldots \ldots \ldots$ & $\ldots \ldots$ & $\ldots \ldots$ & $\ldots \ldots$ & $\begin{array}{c}-4.0295 \\
(0.0044) \\
{[0.018]}\end{array}$ & $\begin{array}{c}-4.6689 \\
(0.0058) \\
{[0.022]}\end{array}$ & $\begin{array}{l}0.6151 \\
(0.0073) \\
{[0.028]}\end{array}$ \\
\hline 16 & 10 & $\ldots \ldots$ & $\ldots \ldots$ & $\ldots \ldots$ & $\ldots \ldots$ & $\ldots \ldots \ldots$ & $\ldots \ldots$ & $\begin{array}{c}-1.0111 \\
(0.0056) \\
{[0.017]}\end{array}$ & $\begin{array}{c}-1.6651 \\
(0.0056) \\
{[0.016]}\end{array}$ & $\begin{array}{c}0.6092 \\
(0.0066) \\
{[0.019]}\end{array}$ \\
\hline $\begin{array}{l}\text { Apr } \\
1993\end{array}$ & & $\begin{array}{c}0.1935 \\
(\operatorname{Tan} 93)\end{array}$ & $\cdots \cdots$ & $\ldots \ldots \ldots$ & $\ldots \ldots$ & $\ldots \ldots \ldots$ & $\ldots \ldots$ & $\ldots \ldots \ldots$ & $\ldots \ldots \ldots$ & $\ldots \ldots$ \\
\hline 18 & 39 & $\ldots \ldots$ & $\ldots \ldots$ & $\ldots \ldots \ldots$ & $\ldots \ldots$ & $\ldots \ldots \ldots$ & $\ldots \ldots$ & $\begin{array}{c}-1.7897 \\
(0.0137) \\
{[0.120]}\end{array}$ & $\ldots \ldots \ldots$ & $\ldots \ldots$ \\
\hline 19 & 57 & $\ldots \ldots \ldots$ & $\ldots \ldots+$ & $\ldots \ldots \ldots$ & $\ldots \ldots \ldots$ & $\ldots \ldots \ldots$ & $\ldots \ldots$ & $\begin{array}{c}-4.8285 \\
(0.0089) \\
{[0.098]}\end{array}$ & $\ldots \ldots \ldots$ & $\ldots \ldots$ \\
\hline 20 & 57 & $\ldots \ldots$ & $\ldots \ldots \ldots$ & $\ldots \ldots \ldots$ & $\ldots \ldots$ & $\ldots \ldots \ldots$ & $\ldots \ldots$ & $\begin{array}{c}-4.8212 \\
(0.0102) \\
{[0.241]}\end{array}$ & $\ldots \ldots \ldots$ & $\ldots \ldots \ldots$ \\
\hline
\end{tabular}

4m Photometric Calibration

\begin{tabular}{|c|c|c|c|c|c|c|c|c|c|c|}
\hline \multirow[b]{2}{*}{ Run } & \multirow[b]{2}{*}{$\begin{array}{l}\text { \# of } \\
\text { stds }\end{array}$} & \multicolumn{3}{|c|}{ Ex linction Goellicients } & \multicolumn{3}{|c|}{ Iransformation slopes } & \multicolumn{3}{|c|}{ 'Iransformation Zero Points } \\
\hline & & $\mathrm{k}_{\mathrm{B}}$ & $\mathrm{k}_{\mathrm{R}}$ & $\mathrm{k}_{\mathrm{B}-\mathrm{r}}$ & $\begin{array}{l}\alpha_{\mathrm{B}} \\
(\sigma)\end{array}$ & $\begin{array}{l}a_{\mathrm{R}} \\
(\sigma)\end{array}$ & $\begin{array}{c}\alpha_{B-R} \\
(\sigma)\end{array}$ & $\begin{array}{l}\zeta_{\mathrm{B}} \\
(\sigma)\end{array}$ & $\begin{array}{l}\zeta_{\mathrm{R}} \\
(\sigma)\end{array}$ & $\begin{array}{c}\zeta_{\mathrm{B}}-\mathrm{R} \\
(\sigma)\end{array}$ \\
\hline $\begin{array}{l}\text { May } \\
1985\end{array}$ & 51 & $\begin{array}{l}0.23 \\
(\mathrm{cun}\end{array}$ & $\begin{array}{l}0.10 \\
\text { ical Kal }\end{array}$ & $\begin{array}{c}0.13 \\
\text { values) }\end{array}$ & $\begin{array}{c}0.0717 \\
(0.1727)\end{array}$ & $\begin{array}{l}-0.0022 \\
(0.0245)\end{array}$ & $\begin{array}{c}1.0829 \\
(0.0105)\end{array}$ & $\begin{array}{c}-2.3307 \\
(0.0196)\end{array}$ & $\begin{array}{c}-2.6612 \\
(0.0277)\end{array}$ & $\begin{array}{c}0.3 .581 \\
(0.0094)\end{array}$ \\
\hline
\end{tabular}


The FITPARAMS task was used to perform an interactive linear least squares fit, which allows bad observations to be flagged and rejected. The fit was performed three times for each observing run. On the first iteration, the best estimate of the extinction was found by fitting the entire data set (all standards observed on a given run) and allowing $k, \alpha$, and $\zeta$ to vary. The extinction was then set to this value and the slope and zero point were fit again, to obtain the value of the slope for the entire run. The final fit was performed holding both the extinction coefficient and the slope constant while determining the zero points for each night of the run.

For the May 85 run, a common solution was determined for all 3 nights as there were too few observations of standard stars to allow an accurate nightly zero point determination. Also, equations 2.2 to 2.4 were changed by replacing $I$ with $R$. In Apr 93, once again there were an insufficient number of standard star observations. In this case the $\mathrm{Jan}_{a} 93$ observing run extinction coeflicient, $k_{B}$, was adopted. Since only $B$ observations were made during the $\Lambda$ pr 93 run, the equations simplify to

$$
B=b_{p h o t}-k_{B} * X_{B}+\zeta_{B}
$$

which unfortunately removes all color dependenee from the fit.

The extinction coefficients, slopes and zero points for all the runs are listed in Table $4 \mathrm{a}$ and $4 \mathrm{~b}$. The standard deviation of the fit, as well as the r.m.s. of the standards from the fit for zero point are listed. 


\subsubsection{Flux Calibration of Galaxy Images}

The normalized sky-subtracted galaxy images were converted into flux calibrated images with the aid of IMFORI', a FOR'I'RAN programming environment designed to interface with IRAF. This program, CALIB, accesses the relevant header information, namely the run and night of observation, filter, effective airmass, exposure time and sky level from the $B$ and $I$ or $R$-band image. The appropriate pixel scale, extinction and transformation coeflicients and photometric zero points are selected automatically based on this header information. The $(B-I)$ sky color in mag $\operatorname{arcscc}^{-2}$ was calculated from

$$
(B-I)_{s k y}=\alpha_{(B-I)}(b-i)_{0, s k y}+\zeta_{(B-I)},
$$

where the $B$-band instrumental magnitude is given by

$$
b_{0, s k y}=-2.5 \log \left[\frac{C_{B s k y}}{S^{2} t_{B}}\right]-k_{B} * X_{B}+26
$$

with a similar equation for $i_{0, s k y} . C_{B s k y}$ is the sky level in $\Lambda \mathrm{DL}$ (Analog to Digital Units) for the $B$ image, and $\ell_{B}$ and $X_{B}$ are the eflective exposure time and airmass for the $B$ image. These parameters were read directly from the image header. The

plate scalc, $S$, in aresec pixel-1, and the extinction coefficient, $k_{B}$, were selected based on the run and night information in the header. The zero point shift of 26 is chosen to keep magnitudes consistent with the standards measured with PHOT (see equation 2.1).

The sky brightness in each filter was calculated using equation (2.6)

$$
B_{s k y}=\alpha_{B}(B-I)_{s k: y}+b_{0, s k y}+\zeta_{B},
$$


with a similar equation for $I_{s k y}$.

Since the images are in the form

$$
C_{r \in l}(B)=\frac{C_{z a l+s k y}(B)-C_{s k y}(B)}{C_{s k y}(B)}
$$

all of the zero point, pixel scale, and extinction terms are contained in the $B_{\text {sky }}$ term and flux calibrated images can simply be obtained by including the slopes, $\alpha$, in the following way:

$$
F(B)=C_{r c l}(B-I)^{\alpha B^{(x)}(B-I)} C_{r c l}(B) 10^{-B_{* k y} / 2.5},
$$

with a similar equation for $F(I)$, and

$$
F(B-I)=C_{r e l}(B-I)^{\alpha_{(B-r)}} 10^{-(B-I)_{s k y} / 2.5} .
$$

The flux calibrated images were calculated in units of ergs $/ \mathrm{s} / \mathrm{cm}^{2} / A \times 10^{10}$ to avoid roundoff errors due to small numbers. Magnitudes calibrated on the standard system were then calculated using

$$
B=-2.5 \log \left[F(B) \times 10^{10}\right]+25
$$

with similar equations for $I$ and $B-I$. The additive constant of $25=2.5 \log 10^{10}$ is present simply because the flux has been multiplied by a lactor of $10^{10}$.

\subsection{Ellipse Fitting and Radial Profile Extraction}

The one-dimensional, azimuthally averaged radial profiles of the surface brightness

of the galaxies, $\mu(r)$, can be obtained by fitting ellipses to the galaxy isophotes. Two 
fitting algorithms were tested. The first, the ISOPHOTE package in STSDAS, is based on an iterative least squares fit to a Fourier expansion, implemented in GASP (GNlaxy Surface Photometry package) by M. Cawson (Carter 1978; Davis el al., 1985) and described in some detail by Jedrzejewski (1987). This method is widely used in fitting the surlace brightness distributions of elliptical galaxies. The contributions of the various terms in the Fourier series indicate the nature and extent of any deviation from ellipticity. However, it is necessary to use a modificd technique when fitting the light of spiral and irregular galaxies (which have even less uniform profiles), because the ISOPHOTE algorithm often produces an unstable fit (Freudling 1992).

This latter technique involves a fully two-dimensional linear fit of the harmonics to the image. The intensity in this case is parameterized by

$$
I(r, \phi)=\sum_{n=0}^{k} I_{n}(r) \cos \left\{n\left[\phi-\phi_{n}(r)\right]\right\} .
$$

which yields a direct (non-iterative) linear determination of the fit. This fit is then used as the initial value for the usual Fourier series expansion fit as in ISOPHOTE, in order to obtain the ellipticity directly. 'T'his procedure is implemented in GA LPHO'T' which was written by Marijn Franx with some modifications by Inger Jørgensen (Franx, Illingworth \& Heckman 1989; Jørgensen, Franx \& Kjargaard 1992).

Stars and cosmic rays werc masked out using DAOPHO'T (Stetson 1987) based routines called GALFIND and CLASSIFY, which generate several lists of pixels to be avoided in the subsequent fit. The harmonic fit was done using the routine HARMFIT, and a residual image was inspected for any remaining stars or cosmic rays which may have been previously masked by the galaxy image. Most of these are 
automatically marked and masked by the DEVIATE task, which masks any pixel which deviates by more than $\check{5} \times$ r.m.s. from zero in this residual image.

The results from the harmonic fit are then used as the initial values for the ellipse-fitting routine, ELLIPFIT. The ellipses were generally allowed to vary in center, position angle, and ellipticity out to about 100 pixels, and ellipses were fit out to a radius where only $60 \%$ of the points on the ellipse lay within the image. This had the advantage of incorporating the sky dominated regions of the image into the profile, allowing the sky level to bo checked using the task SKYCALC as described in .J $\emptyset$ rgensen et al. (1992). The sky subtraction detailed in $\$ 2.2 .1$ was found to be adequate and no changes were made to the sky level at this point.

In order to fit galaxies as irregular as the ones in this sample, the allowed errors in the shape and position of the ellipse had to be increased from $2 \%$ to $10 \%$; the remaining values for all the tasks were set to their default values.

Most of the irregularity in these galaxies is actually intrinsic to the galaxy (i.e. HII regions, not foreground stars) and contributes to the total light, but must be considered excess to the underlying stellar component that is being fit. Therefore, some modifications were made to the GALPIIOT package. The IIII regions and stars within the galaxy were excluded from the image along with the loreground stars and cosmic rays during the fitting process. An additional task, GETML, was written to allow the profiles to be calculated from the actual galaxy image rather than from the model image produced by ELLIPFIT. This is described below.

After the ellipses had been fit and the resulting residual image inspected for any 
remaining deviant pixels (and refitting performed as needed), the task MODMAGS was used to calculate the surface brightness and total magnitudes as a function of the effective radius, $r_{f f f}=\sqrt{a b}$, where $a$ and $b$ are the semi-major and semi-minor axis lengths, respectively. The task was run three times. First, it used the model intensity calculated from ELLIPFIT (the original version of MODMAGS). These are the profiles to which the exponentials were fitted as described in Chapter 3 and plotted in Figures 361 . Next, profiles were calculated with the actual intensity including any HII regions and stars in the galaxy, masked during the fitting, but here included through GETMU. These are the profiles which are plotted as points in Figures 3-64. Finally, all foreground stars were included as well (again through GETMU), in order to more easily illustrate which parts of the profile were being distorted by the stars which had not been completely masked out.

The profiles were magnitude calibrated by adding a zero point of 25.0 (since the flux calibrated images have been multiplied by $10^{10}$; see equation 2.12 ) and the radius was converted from units of pixels to arcseconds in MODMAGS. The resulting profiles were written to STSI)AS TABLES format tables, which simplifies subsequent manipulation of the data.

\subsection{Comparison with Previous Data}

Surface photometry exists for scveral of the galaxics in this sample, from cither photographic or CCD images. The photographic data include observations of UGC 5423 (Schmidt, Richter \& Karachentseva 1985); UGC 7548, UGC 7596 and UGC 

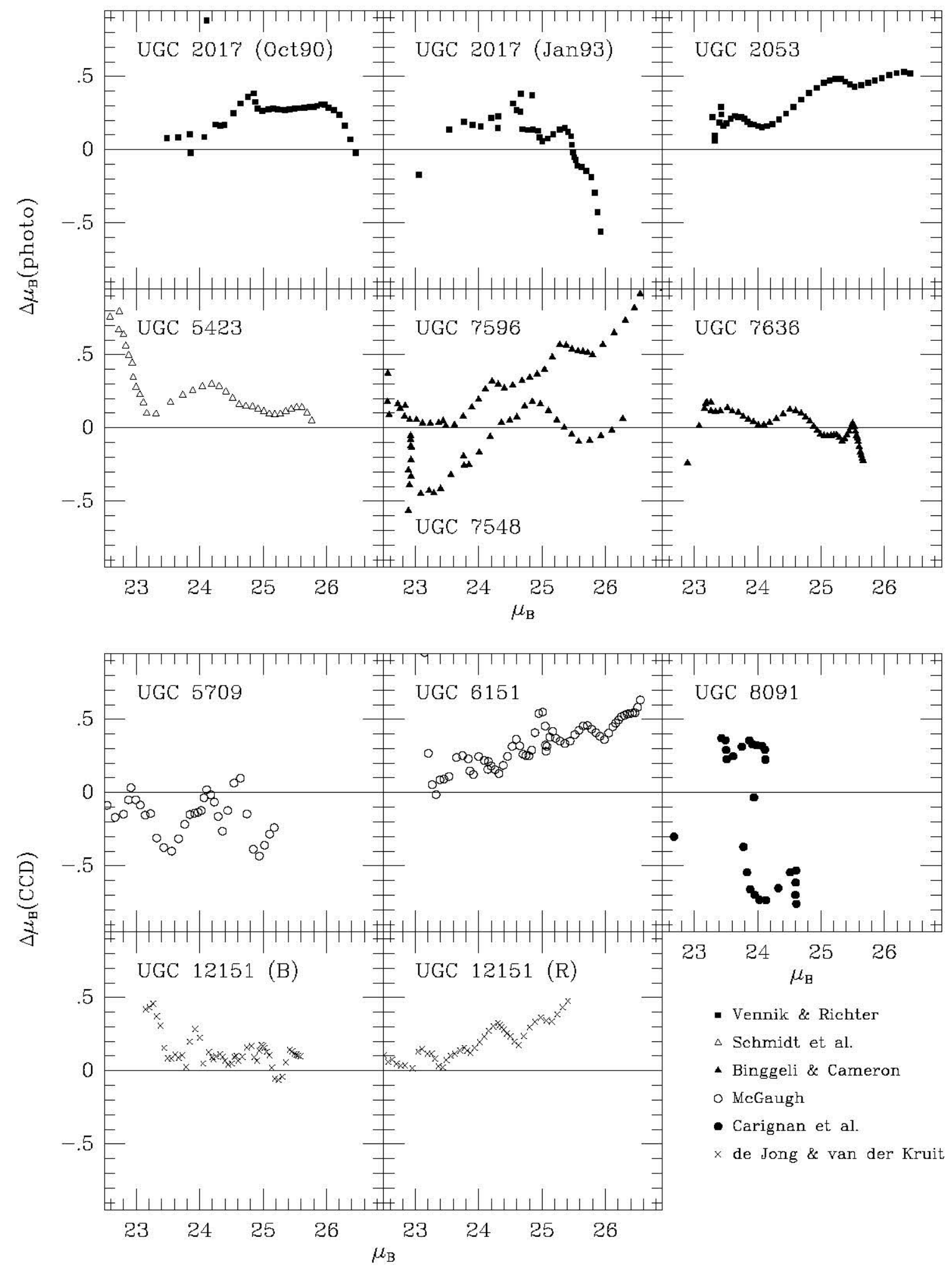

Figure 2: Comparison with other Photographic and (CI) Surface Photometry. $\Delta \mu_{B}=\mu_{B}$ (This work $)-\mu_{B}($ Literature $)$ 
7636 by Binggeli \& Cameron (1993): and UGC 2017 and UGC 2053 by Vennik \& Richter (1994). The comparison with our data, in the sense $\Delta \mu_{B}=\mu_{B}(C C D)-$ $\mu_{\text {photo }}$, is shown in the upper hall of Figure 2. The deviation at extremely low surface brightness levels is attributable to small uncertainties in the sky level, color gradients (since the passbands do not match) and the fact that the profiles are dependent on the reduction technique (choice of "center" of the galaxy). "l'he proximity of NGC 1172 to L $(4) 7636$ surely contributes to the small differenee between the Binggeli \& Cameron data and our data (see Patterson \&. Thuan 1992, for details of the sky subtraction in the case of $\mathrm{UGC} 7636)$.

The surface brightness profiles obtained from CCD $B$-band data is compared with our own data in the lower half of Figure 2. The ECC 5709 and EGC 6151 data are from McGaugh \& Bothun (1993); the UGC 8091 data are from Carignan, Beaulieu \& Freeman (1990); and the LGC 12151 data are from de Jong \& van der Kruit (1994). The intrinsic irregularity of the galaxies (especially UGC 8091) makes it difficult to directly compare fits made using different algorithms. In particular, the choice of which stars to exclude from the fit obviously has a great impact on the final profile. Inder these limitations, it was felt that the agrecment with the previous data (typically, $\Delta \mu_{B}<0.5 \mathrm{mag} \operatorname{arcsec}^{-2}$ ), as illustrated in Figure 2, was satisfactory. 


\subsection{Presentation of the Data}

Immediately following this section is a series of figures which present the inaging data, as well as the results of the ellipse fitting for each galaxy. In Figures 3-63 a greyscale image along with a contour plot are shown for each galaxy for each band in which it was observed. The center of the galaxy from the ellipse fitting, and the scale in arcseconds, is indicated on the contour plot. Below these, the surface brightness profile from the ellipse fitting is given both with (open circles) and without (closed circles) the HII regions and stars in the galaxy included in the total light. The actual fit was made to the $I$-band image with the "HII regions" excluded. This isophotal fit was then used to obtain the profile fit including these "HII regions". The $B$ profile was also obtained using the $I$-band isophotal fit. The line shown is a fit to the indicated region wilhoul "IIII regions"

In Figures 64-71, the ellipticity and cos(40) term, from the ellipse fitting, and the color profile are shown. The ellipticity $(e=1-b / a)$ varies a great deal for small radii, but 11sual becomes stable at large $r_{e f f}$. The adopted value of the ellipticity (the average value of the ellipticity at large radii), used to derive the inclination in Chapter 3, is marked. The variation of the $\cos (1 \theta)$ term indicates to some extent how well the galaxies light is fit by an ellipse at a given isophote level. Deviations of this term from 0 are used to determine whether an elliptical galaxy has "peanutshaped" or "pointy" isophotes. In the present application we use this deviation as a measure of the applicability of elliptical isophotes to these galaxies. If there is a large deviation from 0 at a large $r_{f f f}$, the assumption of an underlying disk is suspect. 
Corrections to the magnitude and HI linewidth (discussed in Chapter 3) based on an assumed inclination are questionable under these circumstances, and these galaxies are excluded from the Tully-Fisher analysis in Chapter 4.

The color profiles were formed from the difference of the average surface brightness (excluding "IIII regions"; actually IIII regions as well as stars present in the galaxy and masked for the ellipse fitting) within an elliptical anmulus in each filter. The ellipses are identical in each filter, having becn determined from the $I$-band image.

Histograms of the size distribution of the "HII regions" are shown in Figures 72 71. The clumpiness of the distribution is caused by the finite pixel size. The radial and azimuthal distribution of these regions are presented in Figures 75-80. The data are presented in this way to give a feel for the distribution of the regions which were excluded from the fit. Because the objects are not restricted to HII regions, it would be misleading to compare these data with that of authors such as van den Bergh (1981) and Strobel, Ilodge \& Kennicutt (1991), who investigate the size distribution of IIII regions with respect to the global properties of the parent galaxies. 
Figures 3-63: Images, Contour Plots and Surface Brightness Profiles of all Galaxies in the Sample. The first 53 (Figures 3-56) figures are of $B$ and $I$ pairs of images of galaxies in the sample, with the remainder being $R$ data and $B$ data with no corresponding $I$ data. All of the images (upper panel) are oriented with North at the top and East to the left.

The contour plots (middle panel) are plotted in steps of $0.5 \mathrm{mag} \operatorname{arcsec}^{-2}$, starting at a contour level of 27.0 for $B$ and $R$ and 25.5 for $I$, unless specifically noted on the figure. The highest contour level shown, 11sually corresponding to the peak surface brightness within the galaxy, is listed. 'I he arrows in the contour plot indicate the center that was used for fitting ellipses, determined from the $I$-band image. The scale for both the images and contour plots is indicated by the scale bar in the contour plot. Also, each of the small tickmarks around the contour and the image plots represent $20^{\prime \prime}$.

The surface brightness profiles panels (at the bottom) show the profiles (•), obtained from GALPIIOT, excluding the IIII regions and stars and the exponential fit (described in Chapter 3 ) over the indicated interval of radius. In addition, the points including the. HII regions and stars within the galaxy, but excluding foreground stars, are plotted (o) for comparison. In nearly cvery casc, the $/$-band image was used in the ellipse fitting. In the few cases where no / data was taken, they were fit to cither the $R-$ (if available) or $B$-band images. 
B
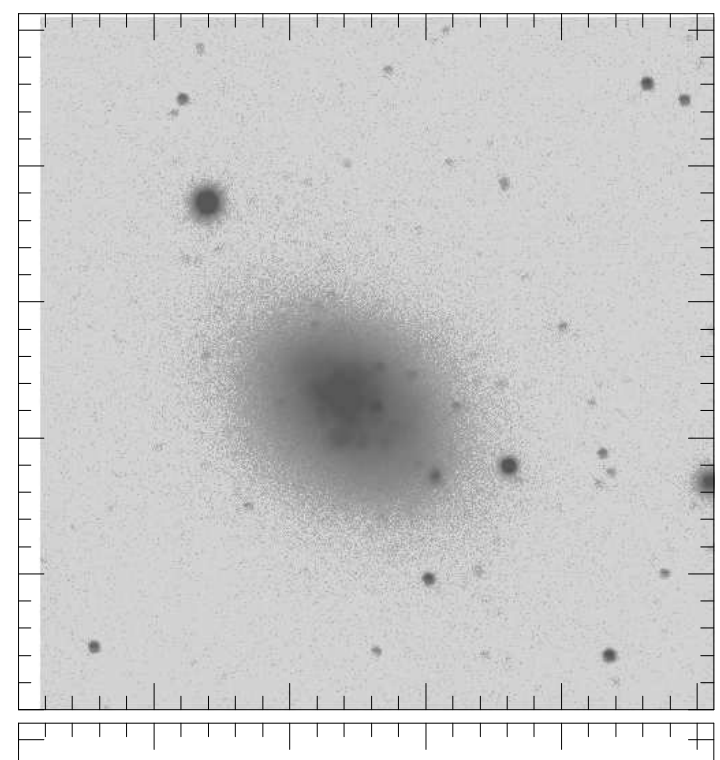
- $\mu_{\text {top }}=22.0$

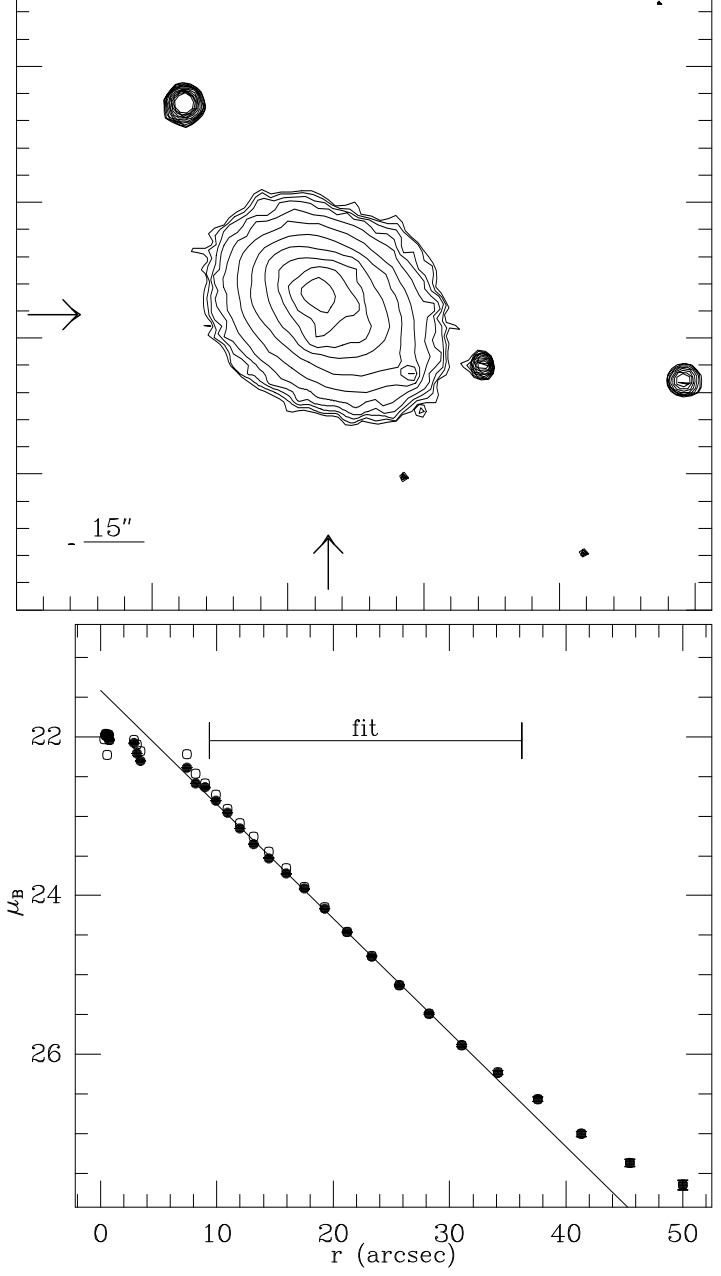

I 42
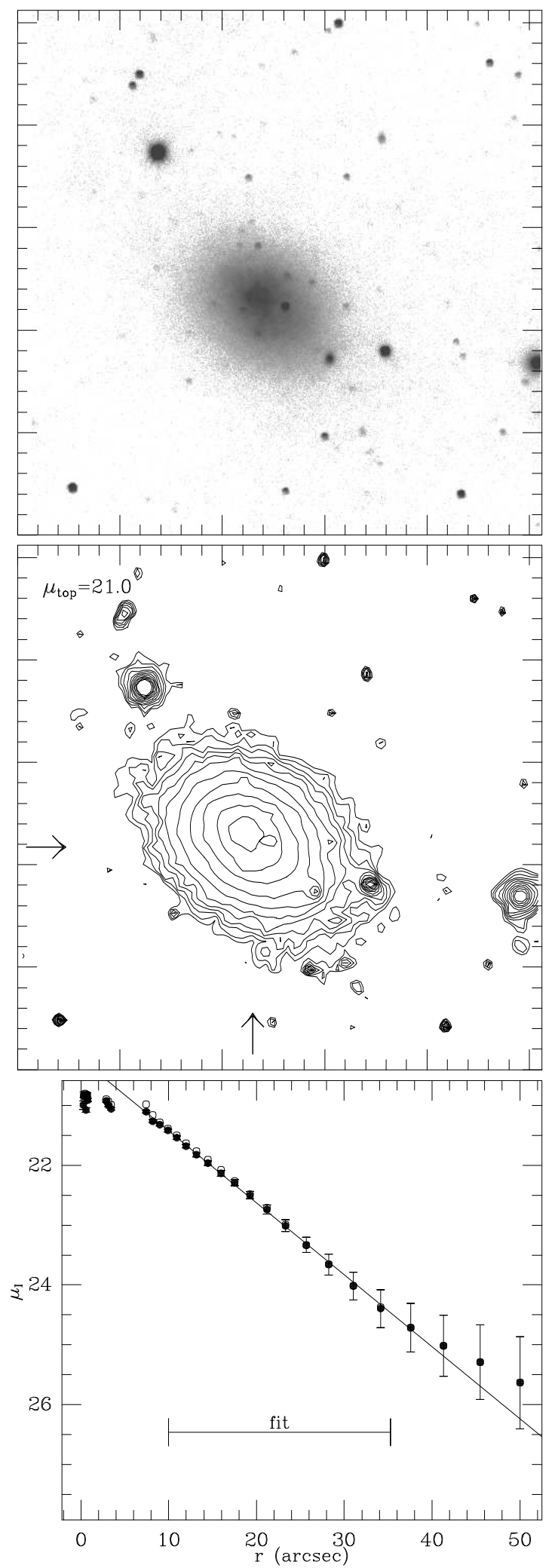

Figure 3: UGC 00031 
B

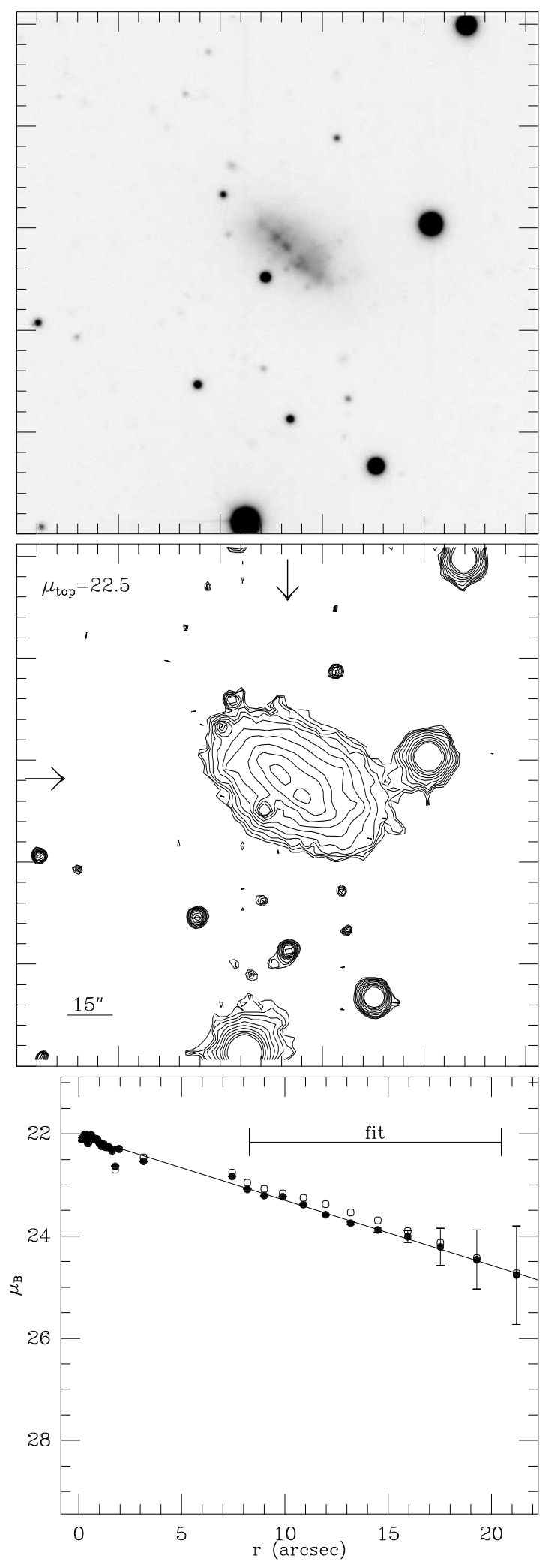

I
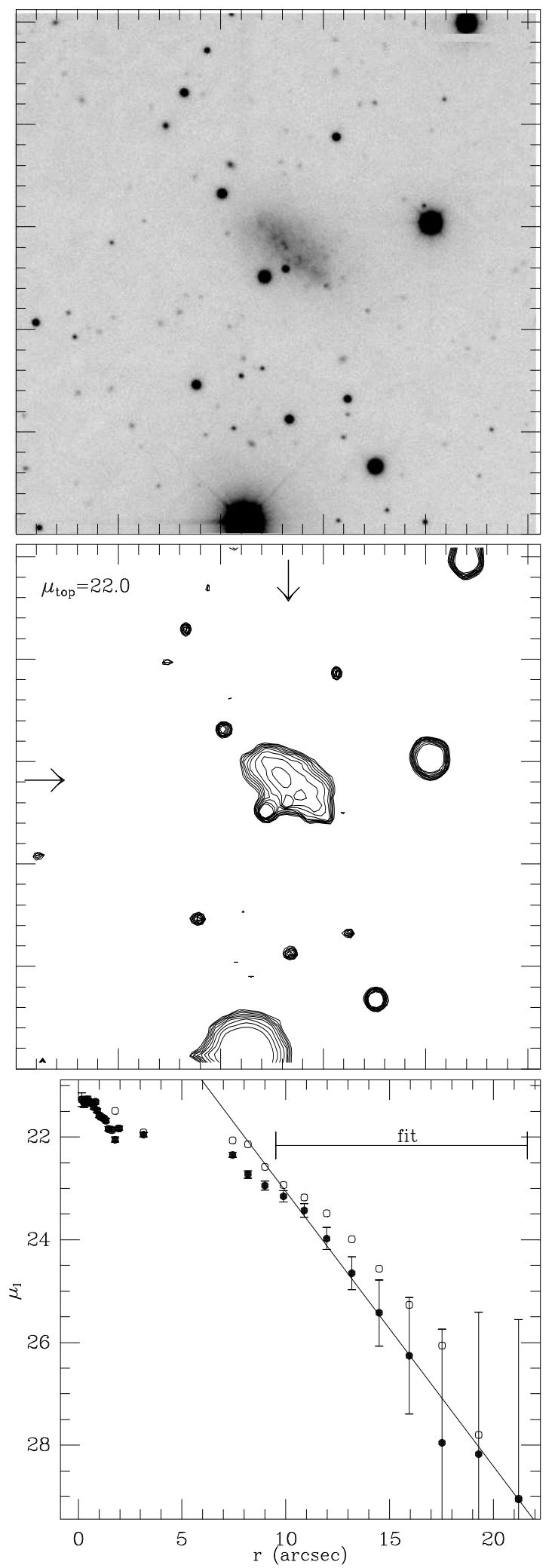

Figure 4: UGC 00063 
B

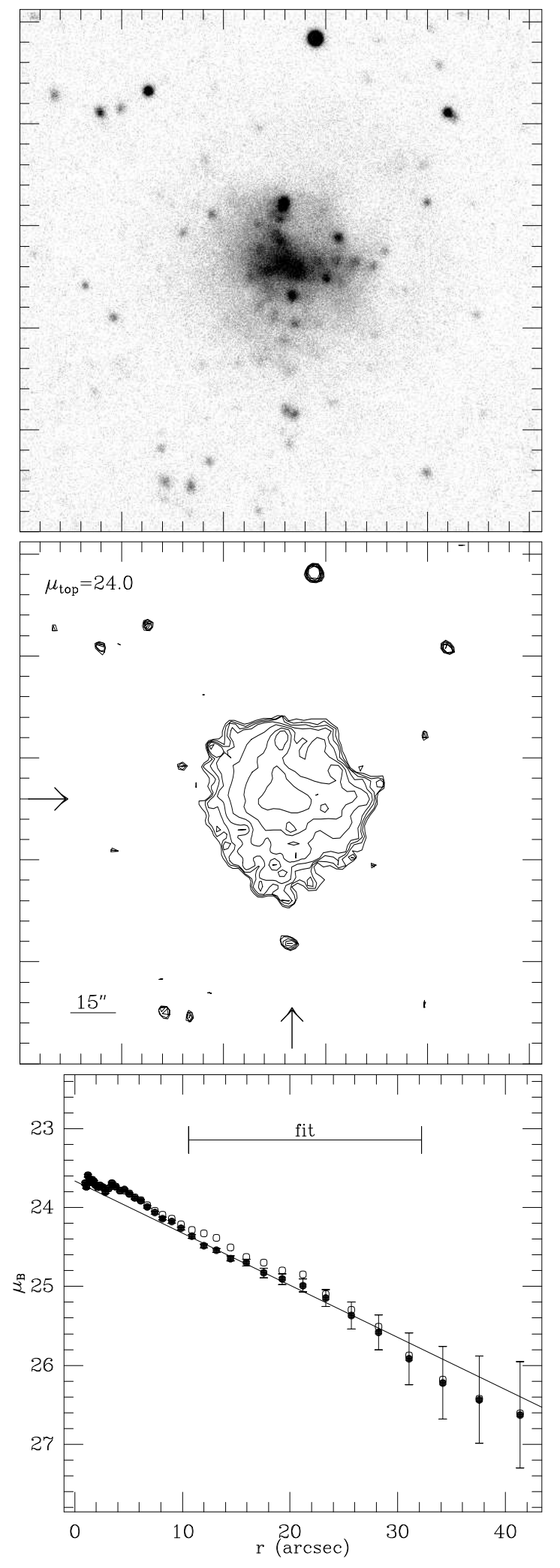

44
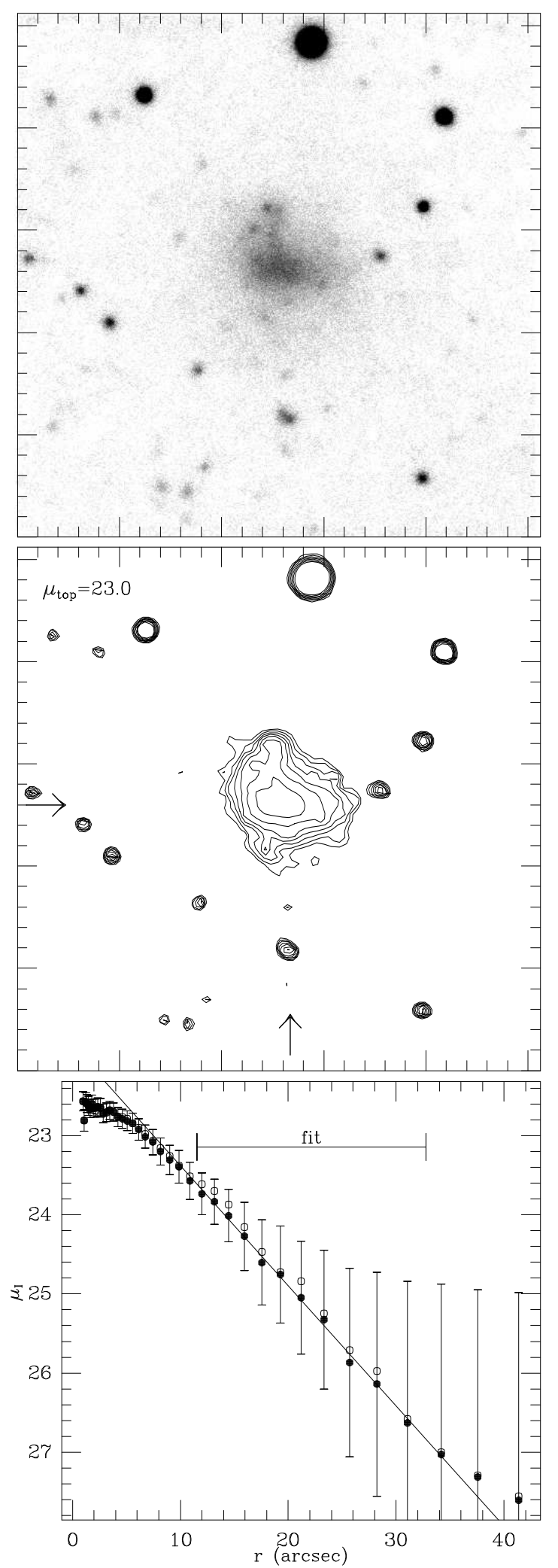

Figure 5: UGC 00300 
B

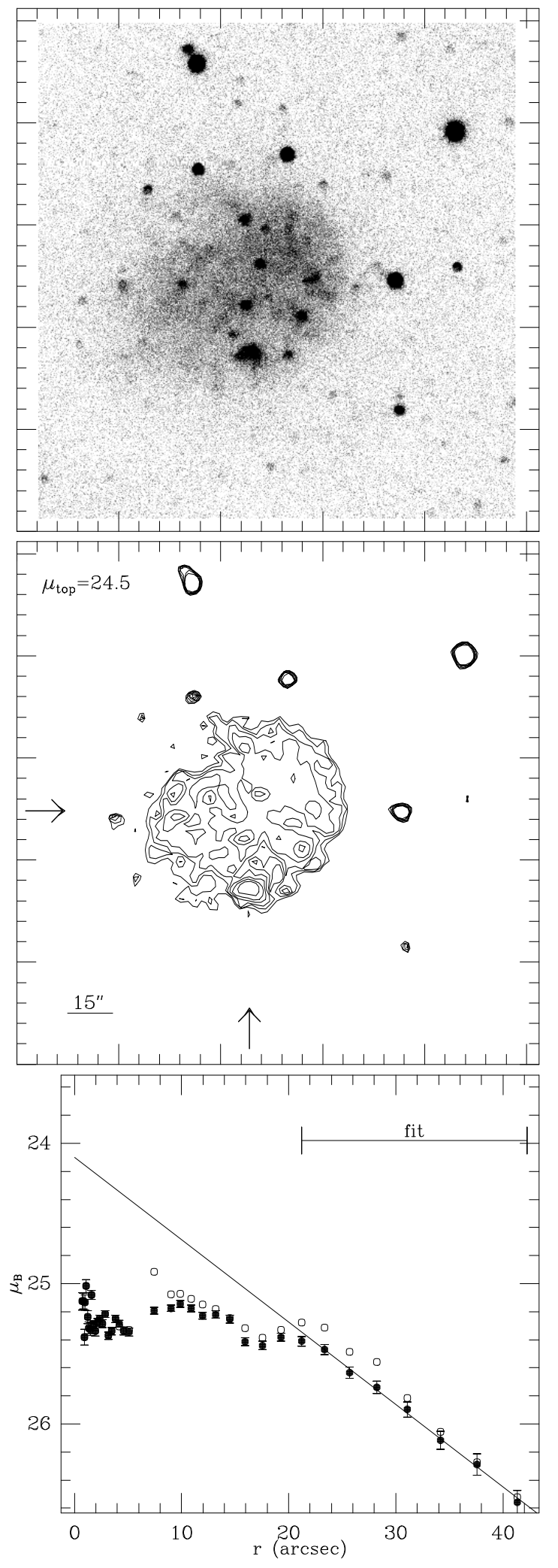

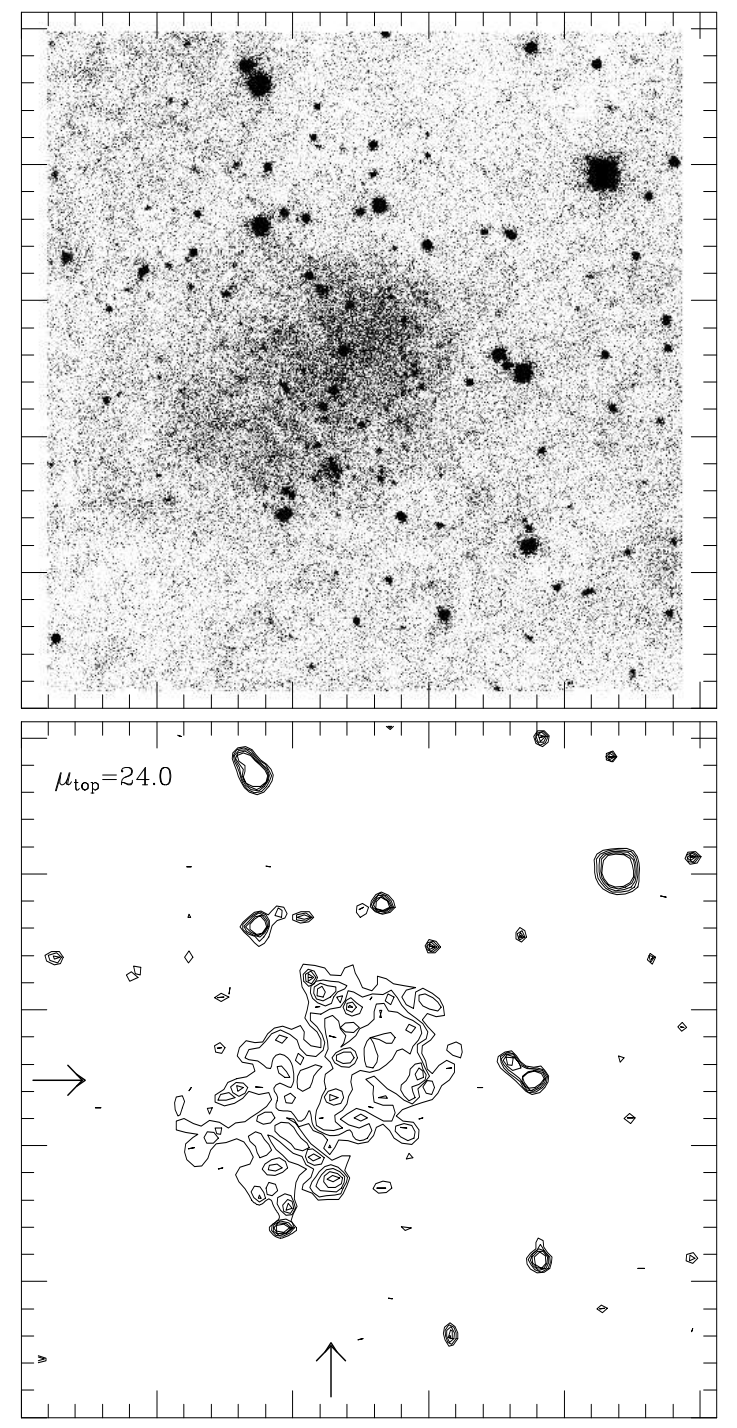

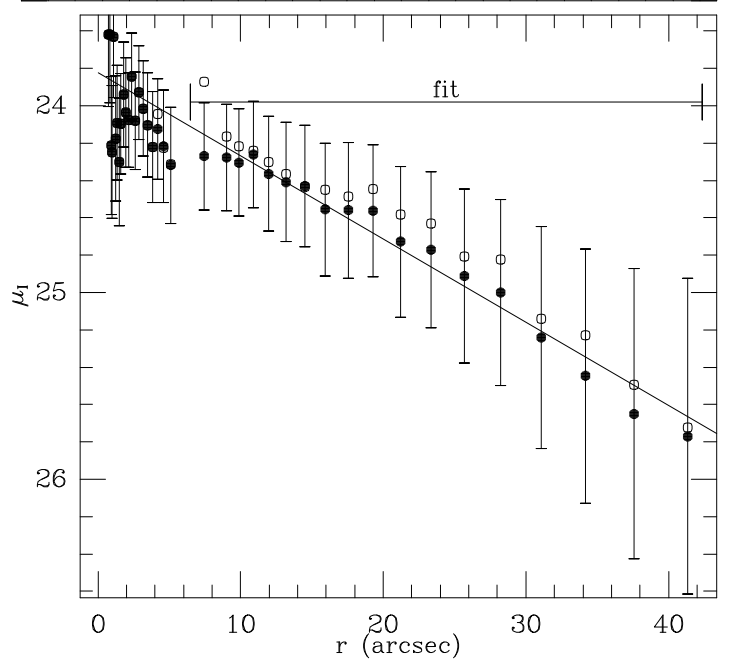

Figure 6: Karachentsev 10 
B
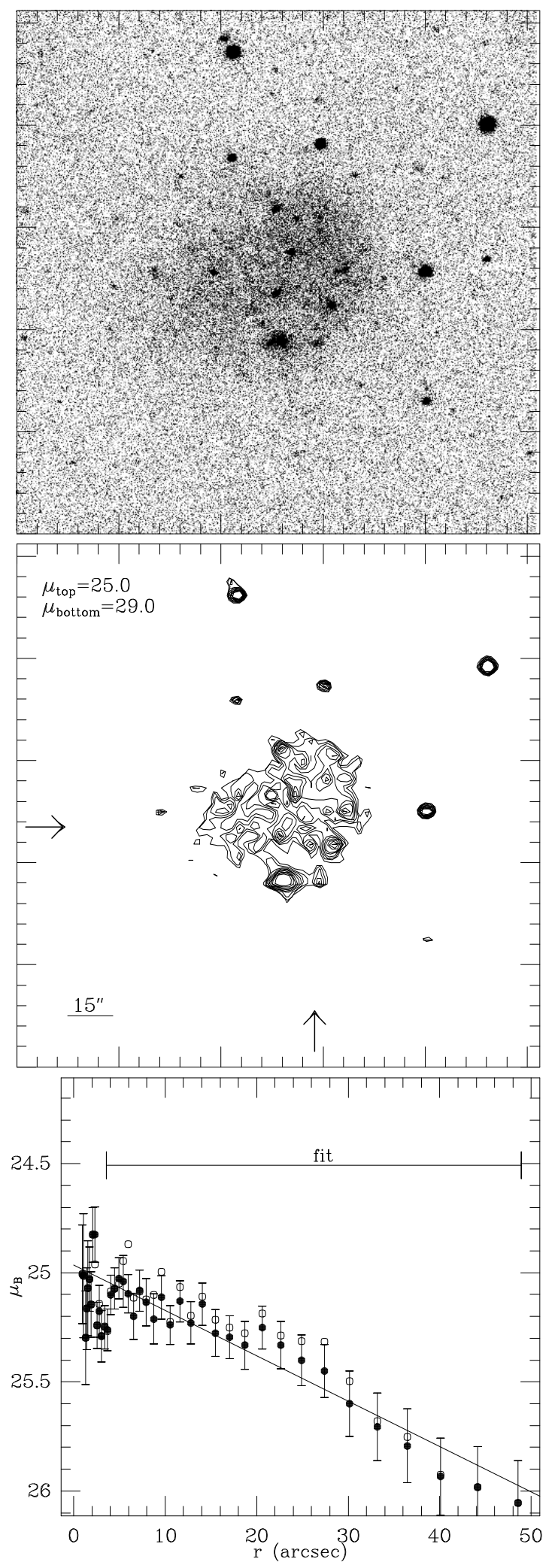

I
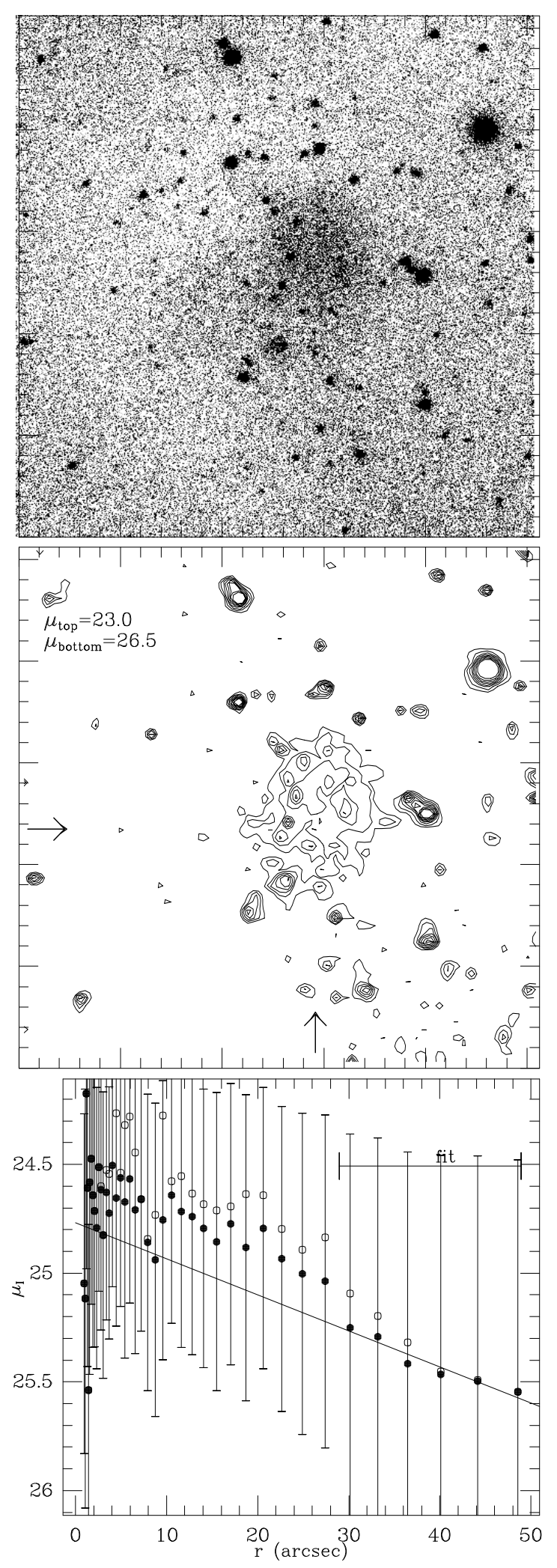

Figure 7: Karachentsev 10 (Jan93) 

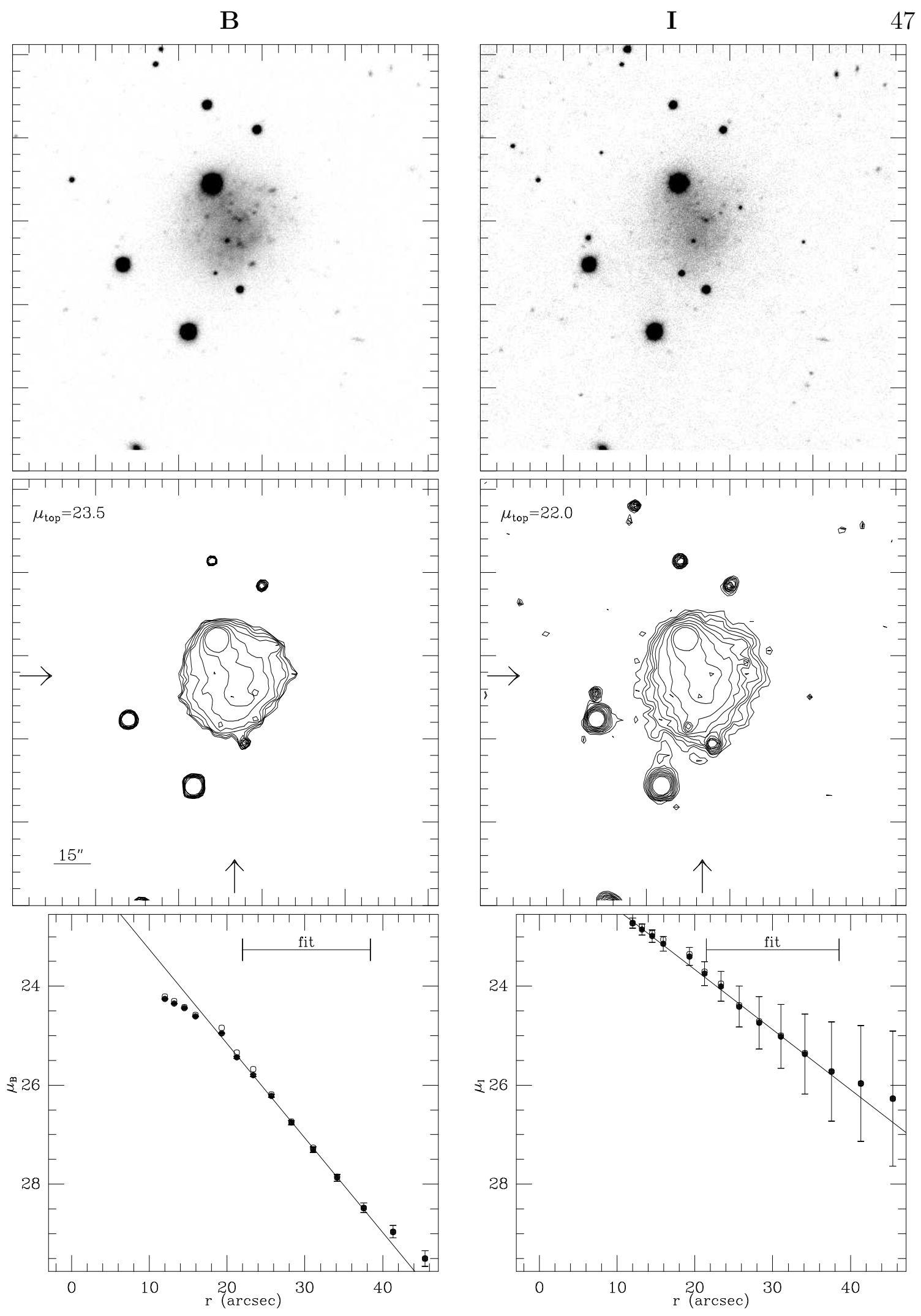

Figure 8: UGC 01981 

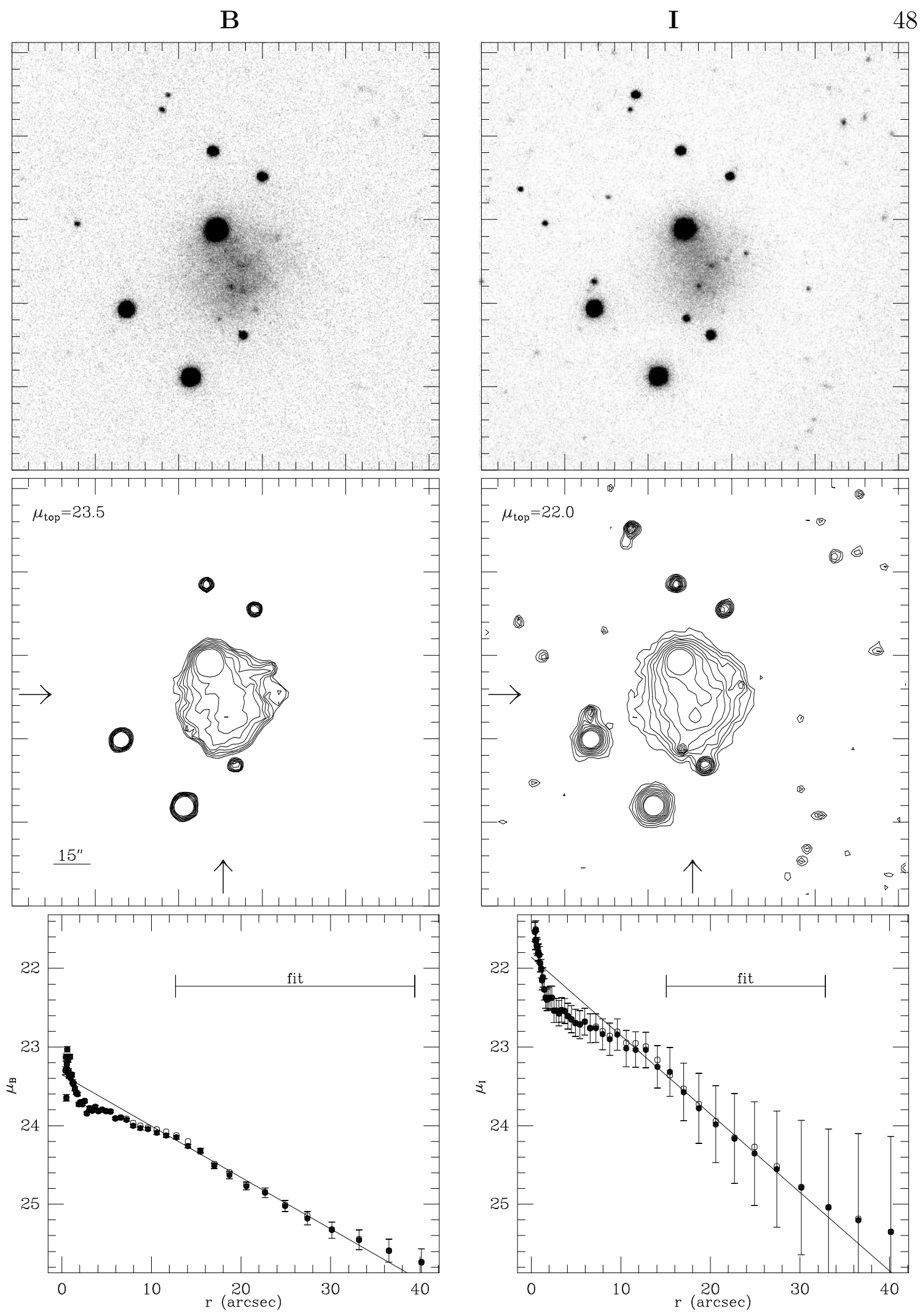

Figure 9: UGC 01981 (Jan93) 
B

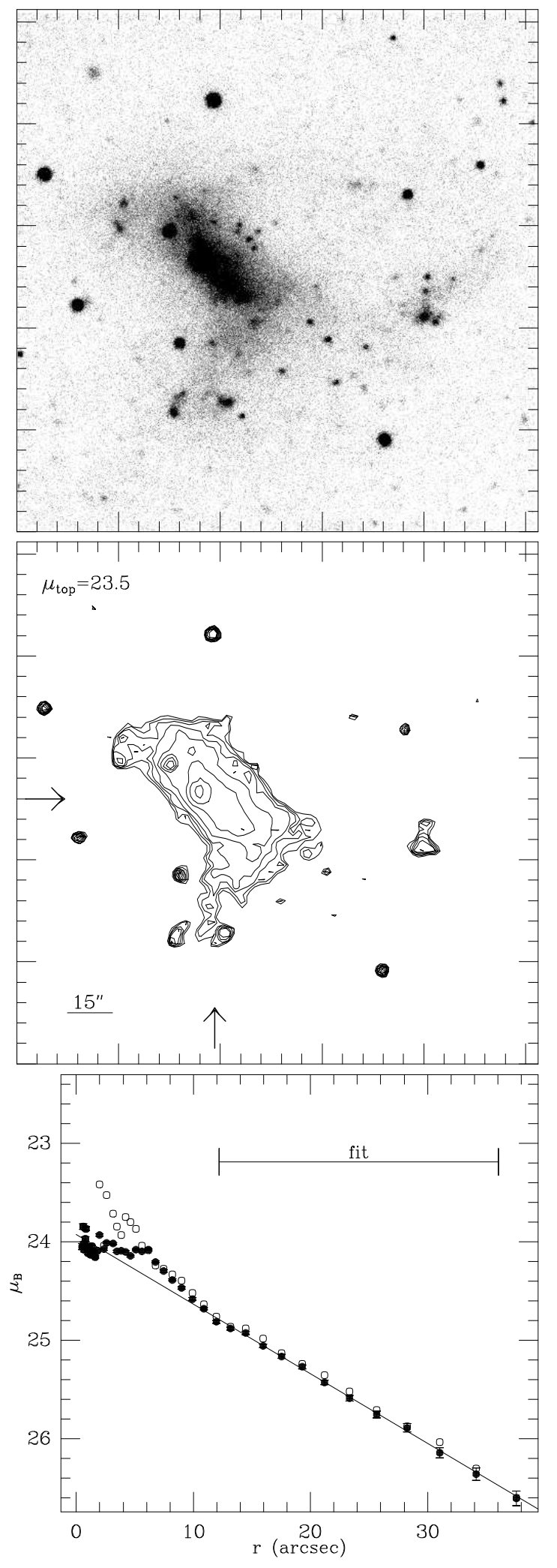

I

49
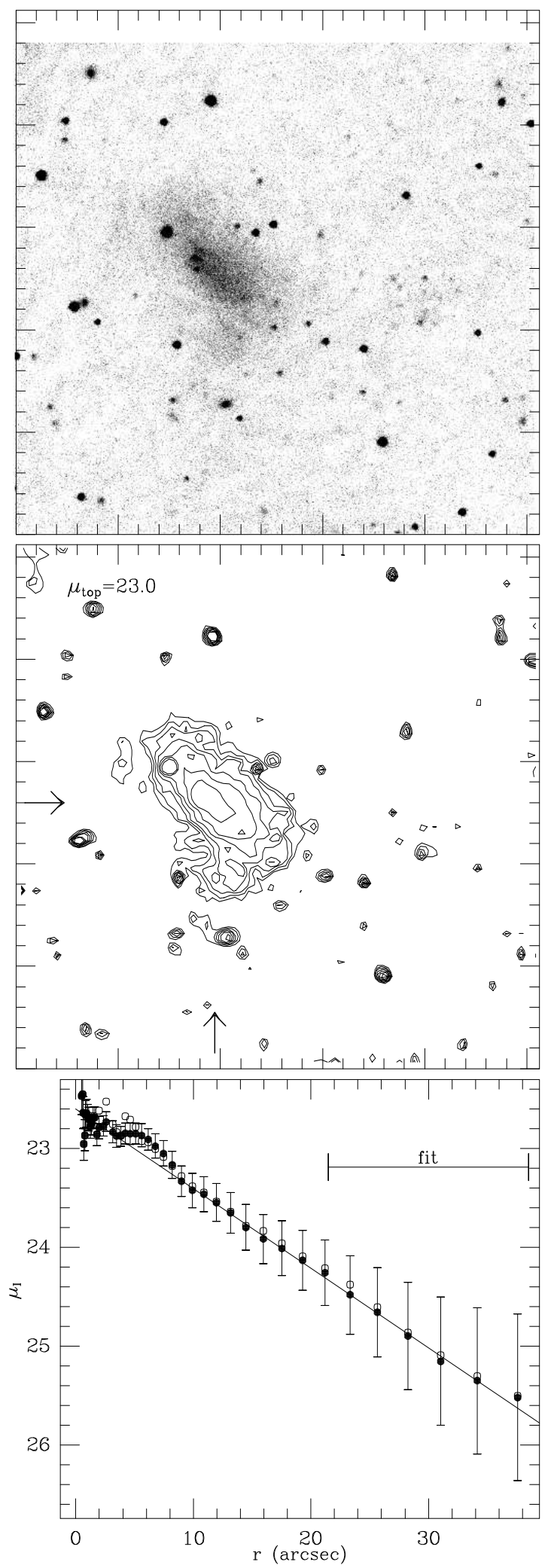

Figure 10: UGC 02017 
B

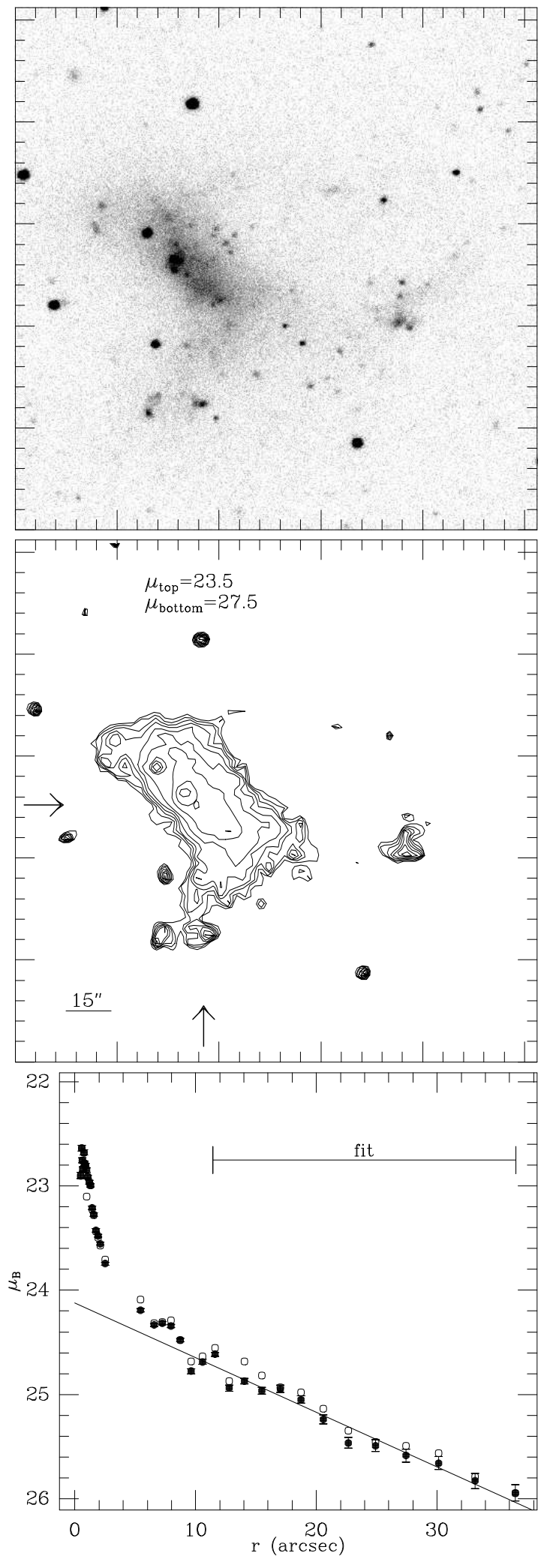

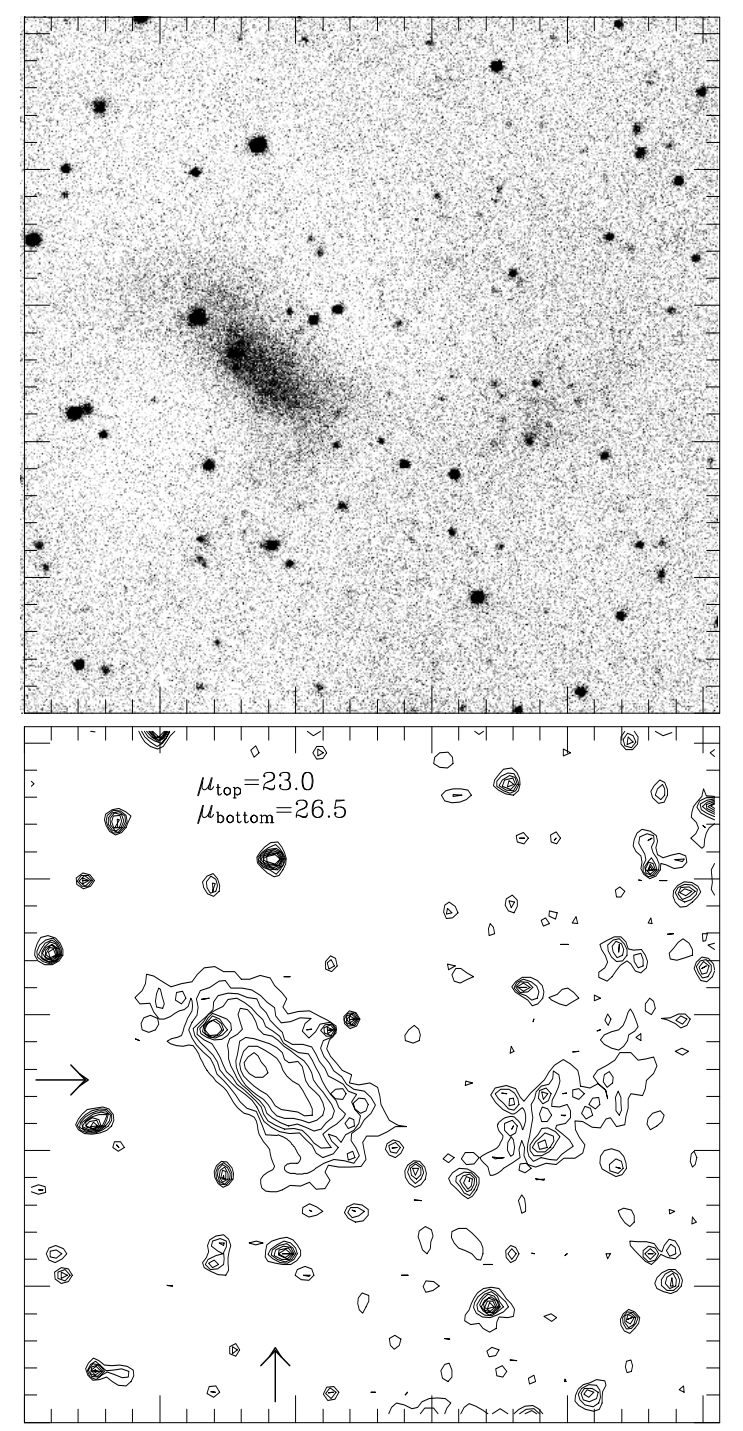

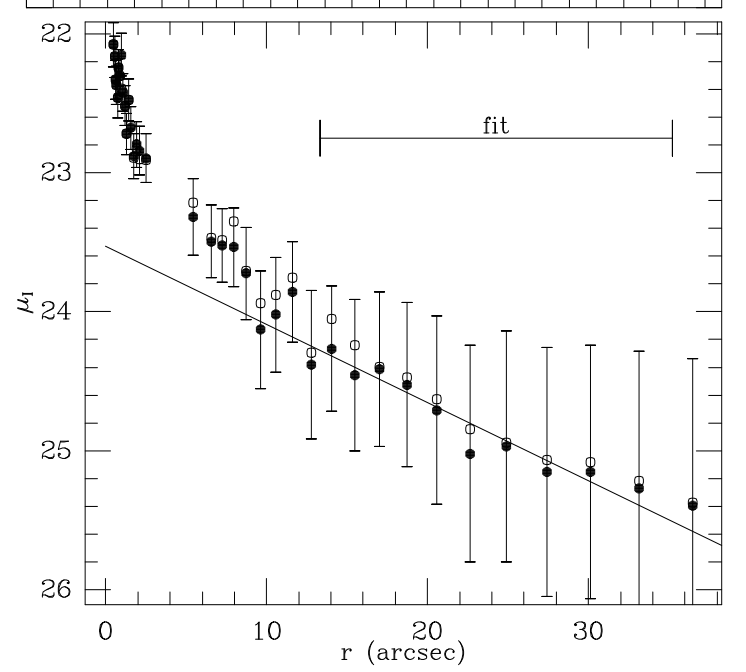

Figure 11: UGC 02017 (Jan93) 
B

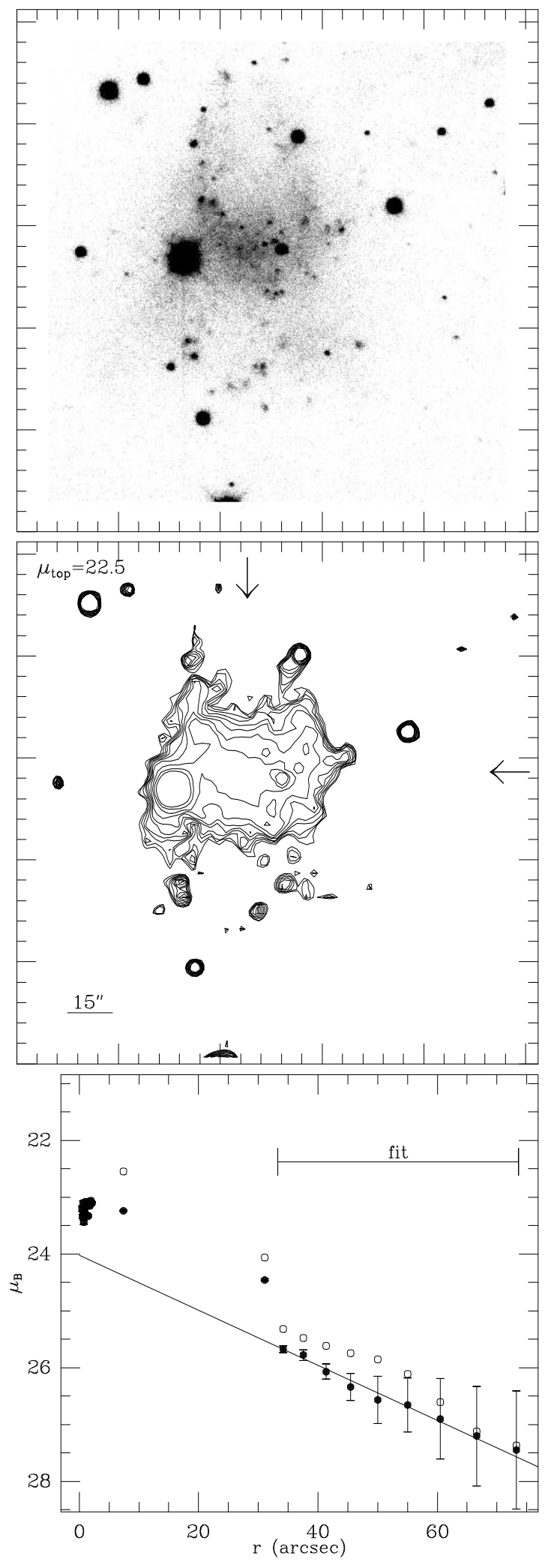

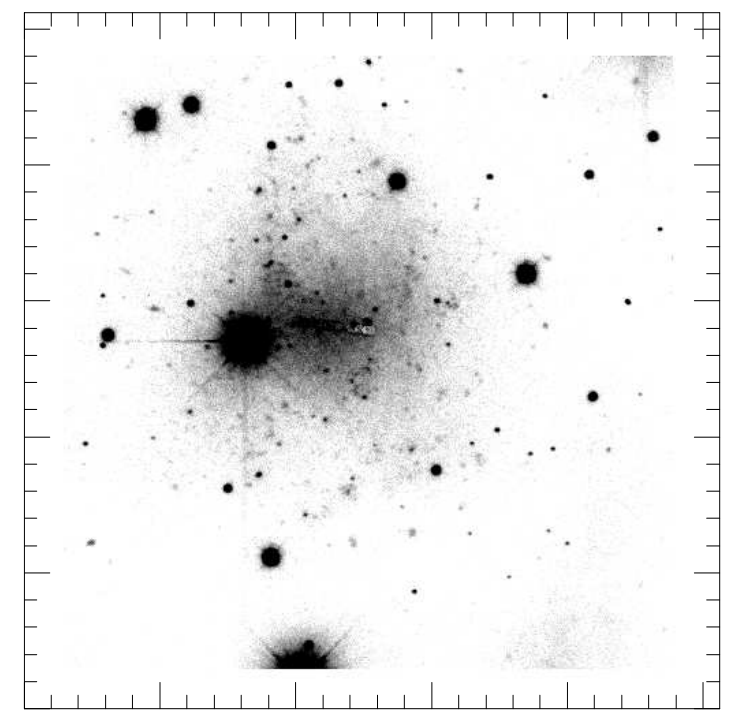
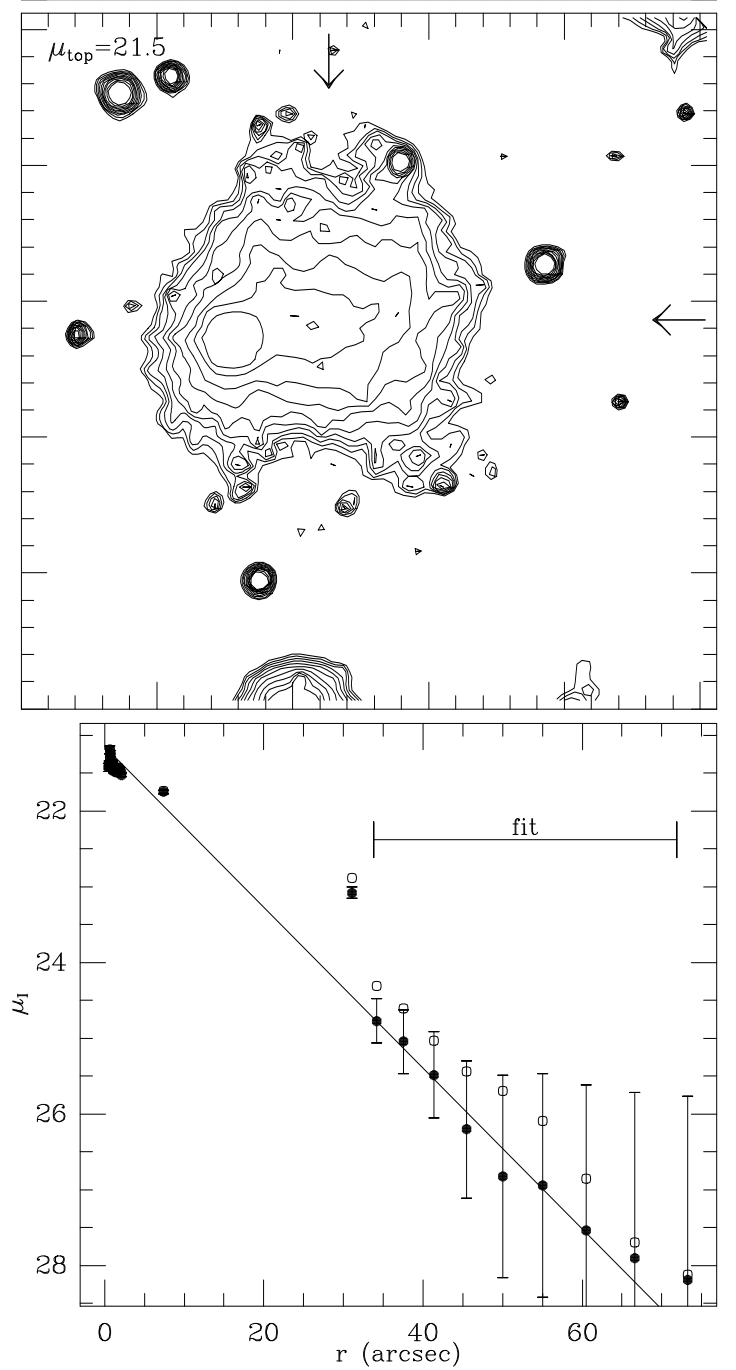

Figure 12: UGC 02034 
B

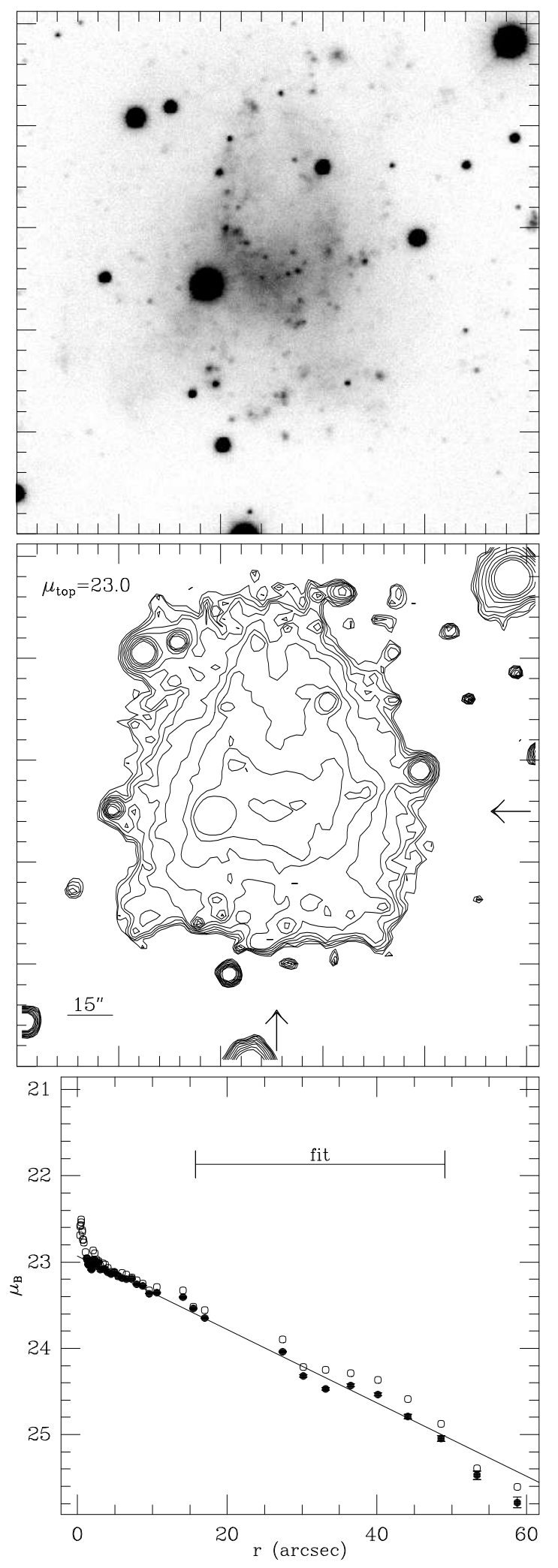

I

52
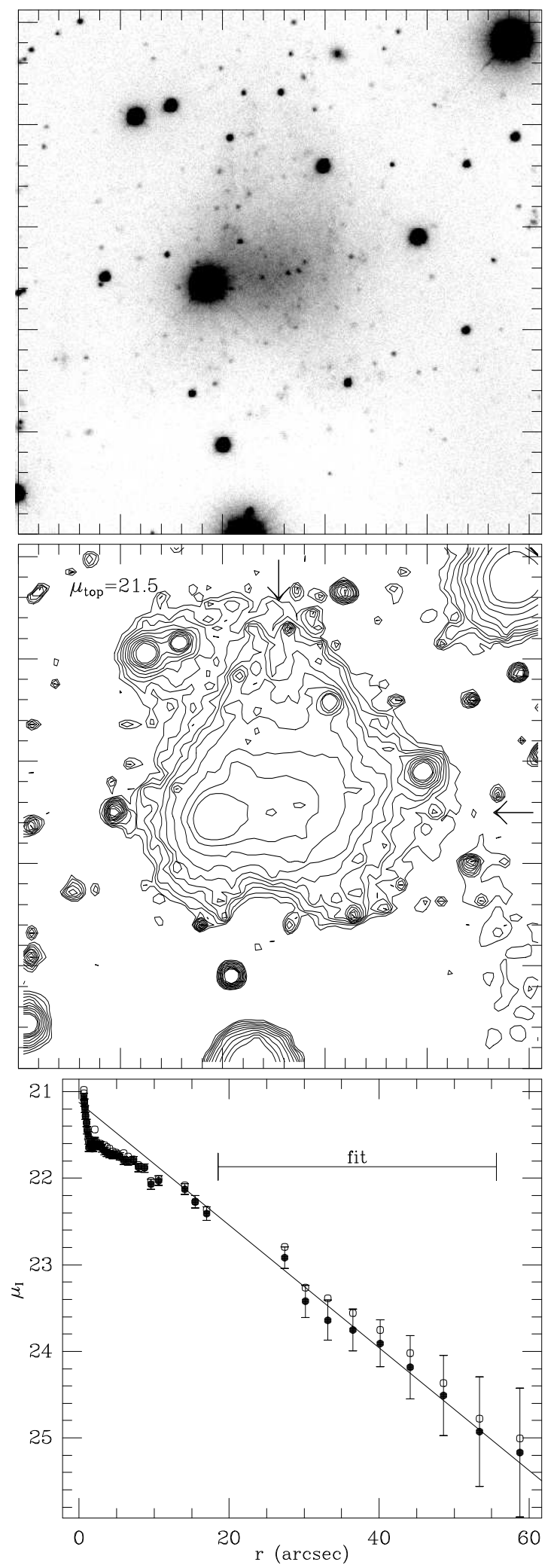

Figure 13: UGC 02034 (Jan93) 
B

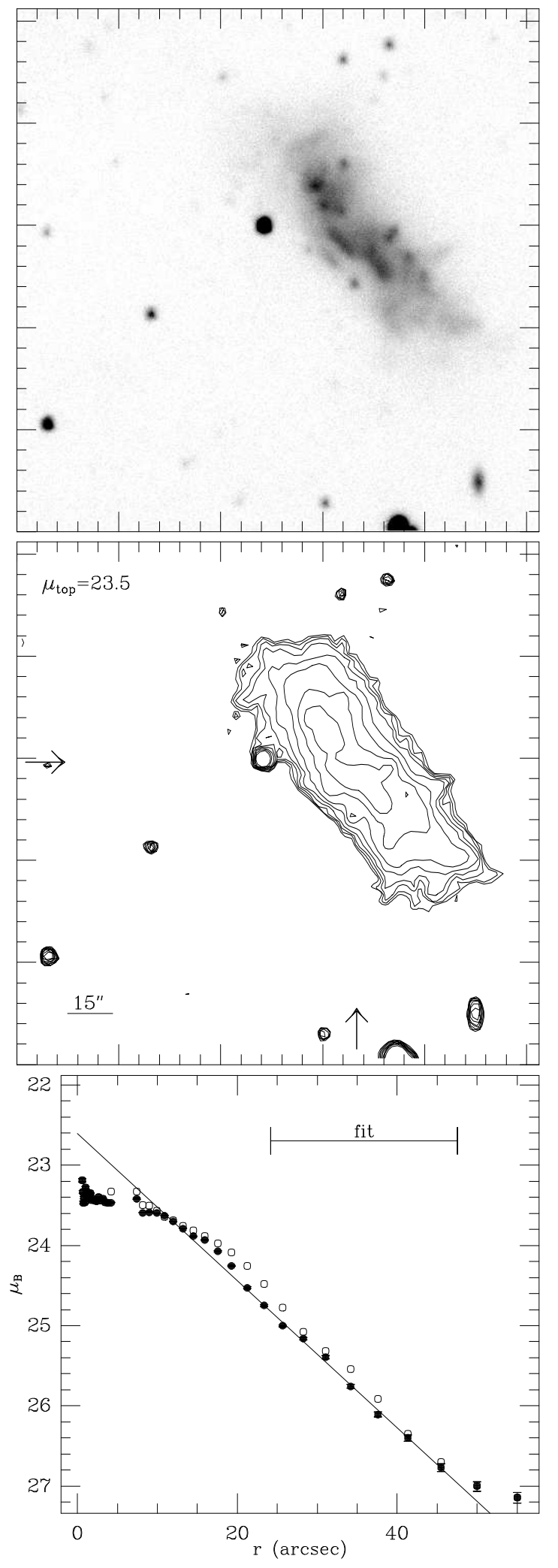

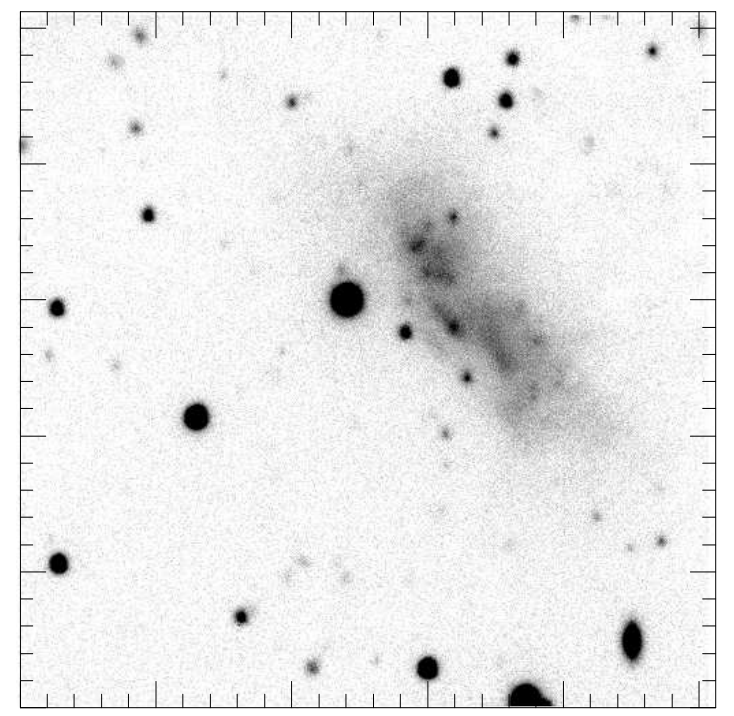
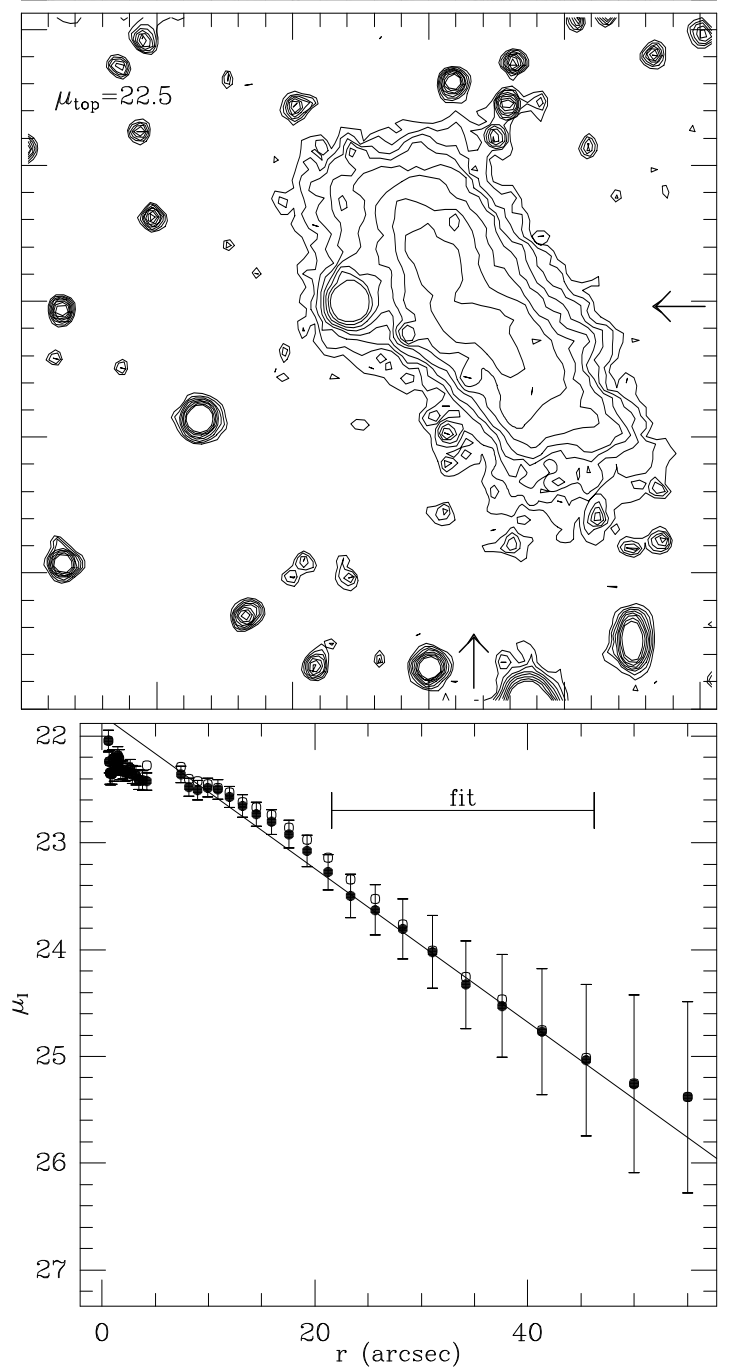

Figure 14: UGC 02053 
B

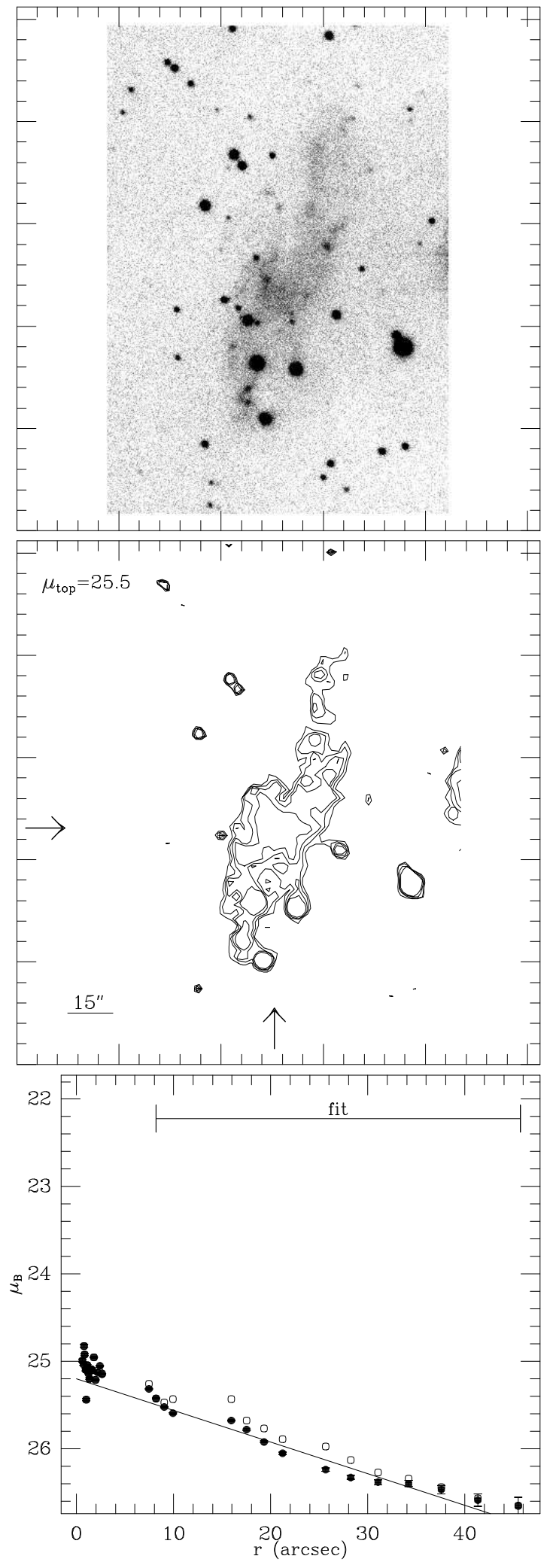

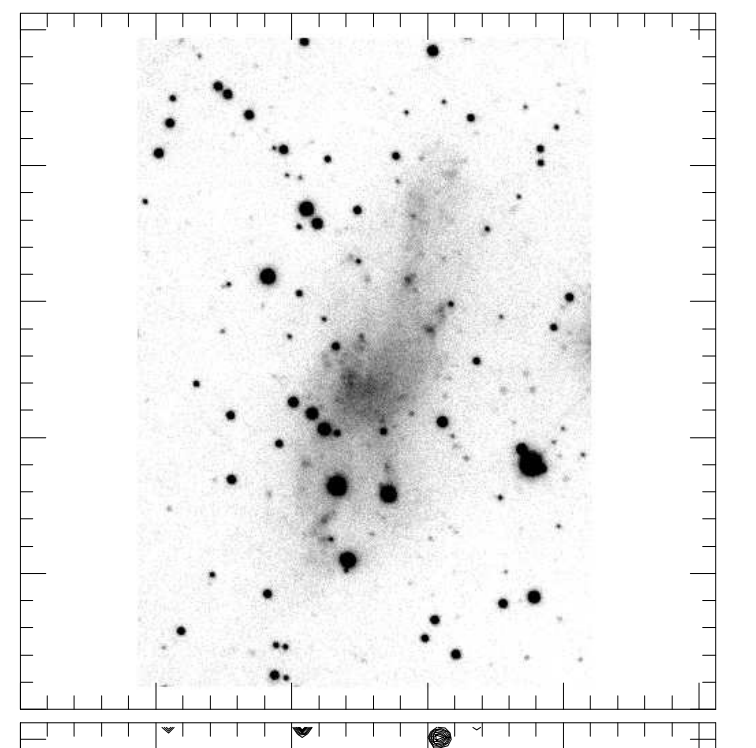
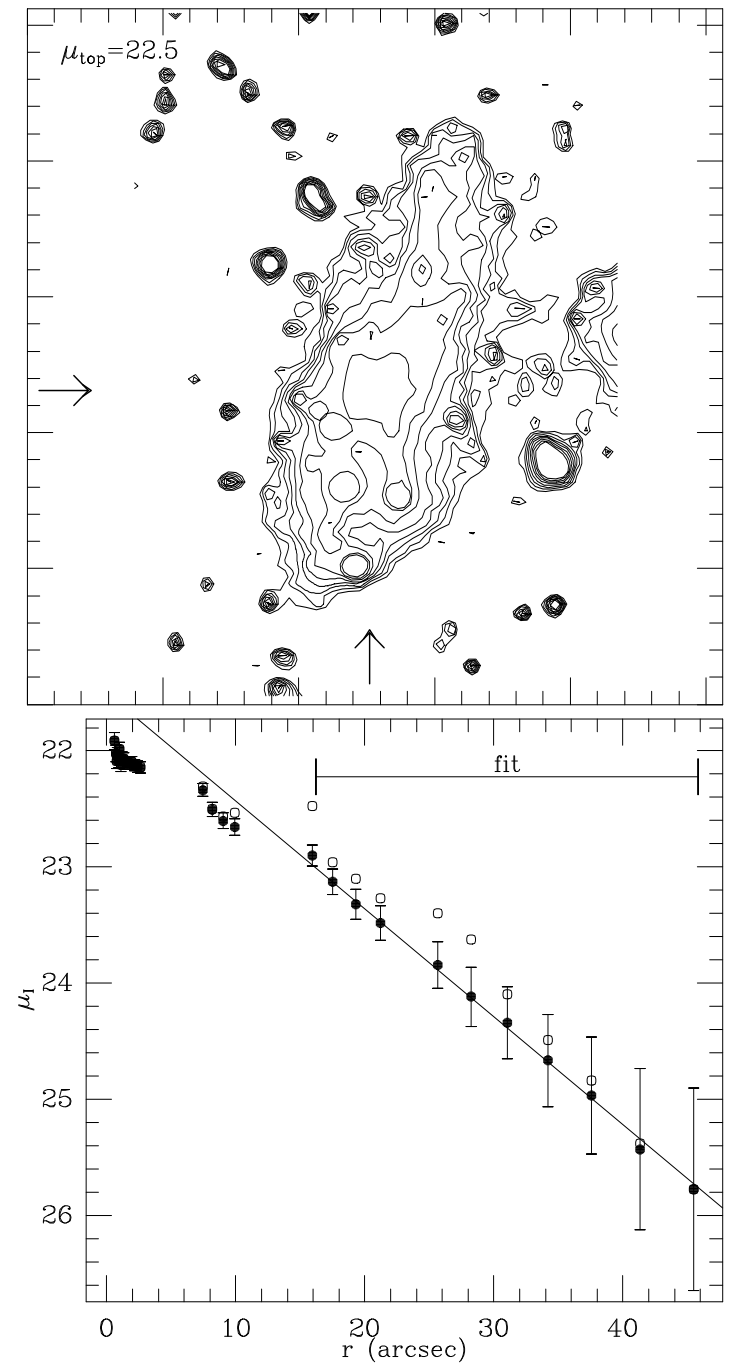

Figure 15: Karachentsev 37 

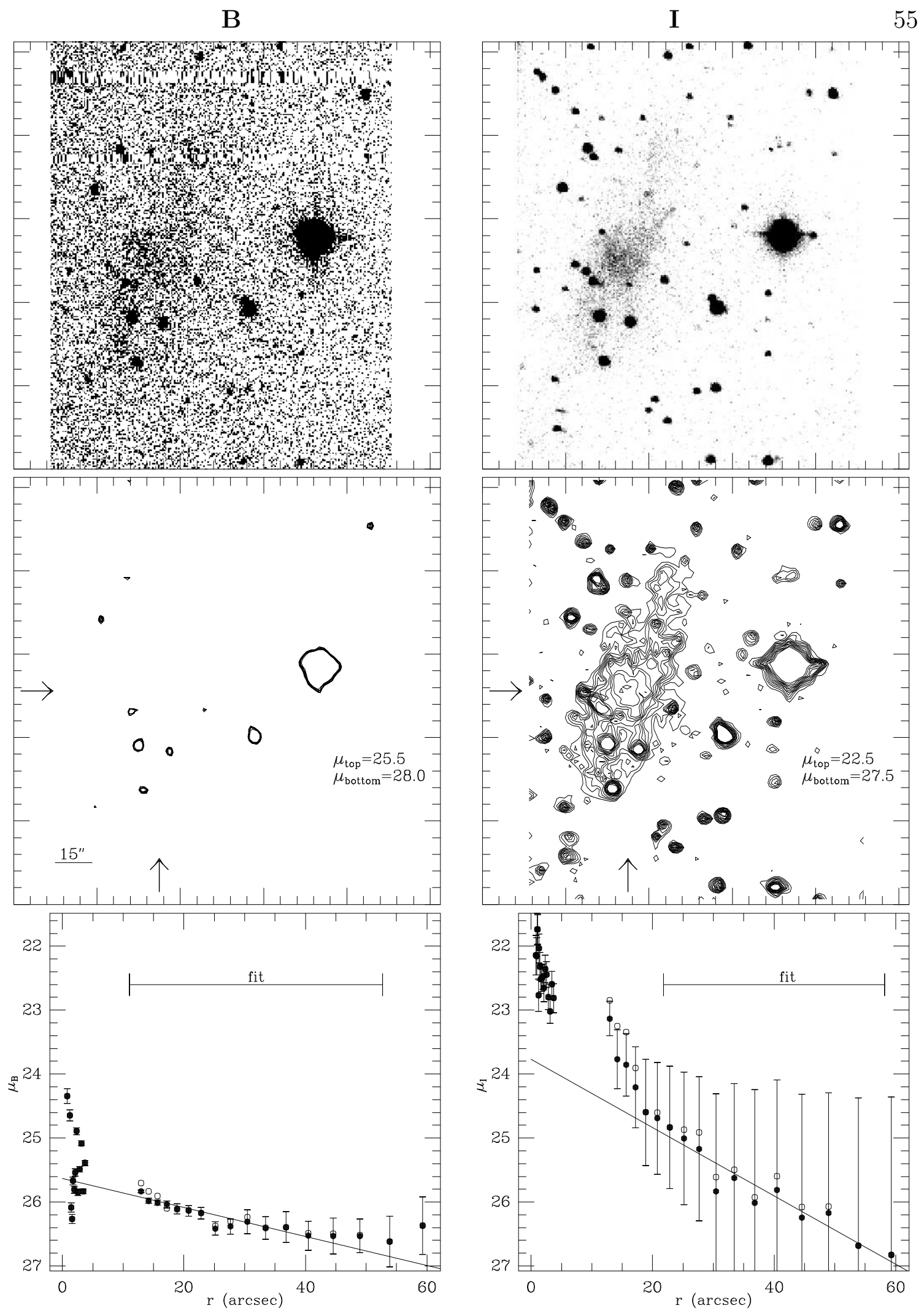

Figure 16: Karachentsev 37 (Sep91) 
B
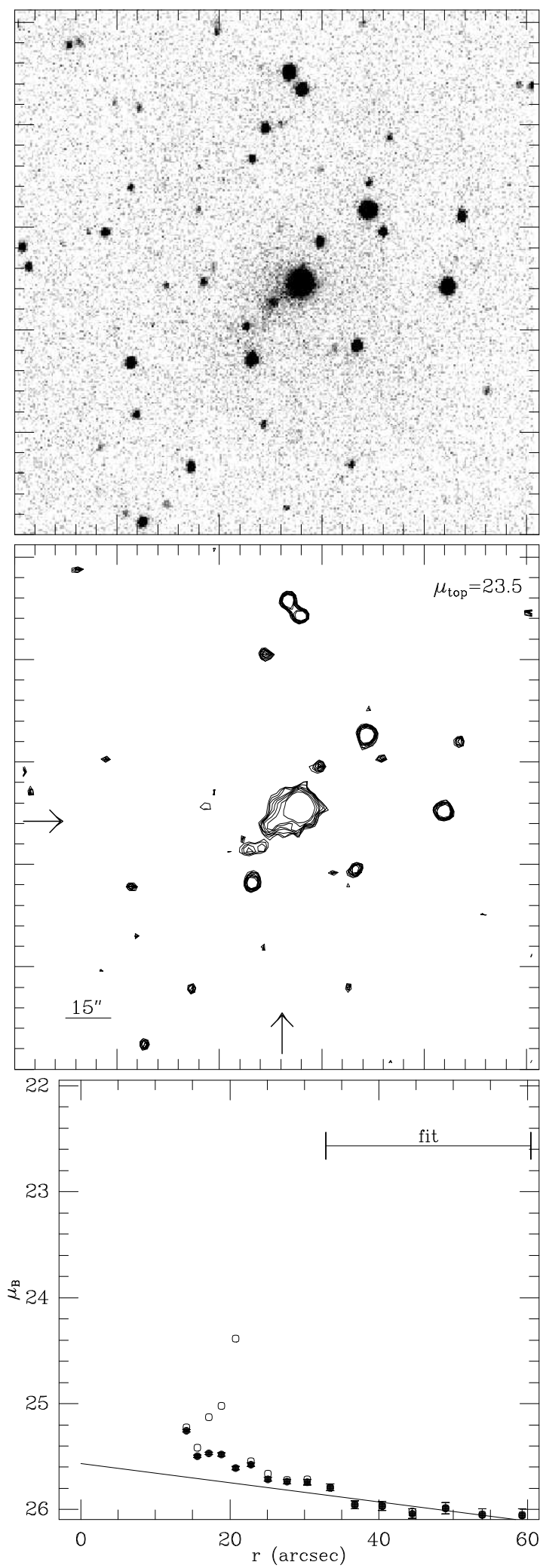

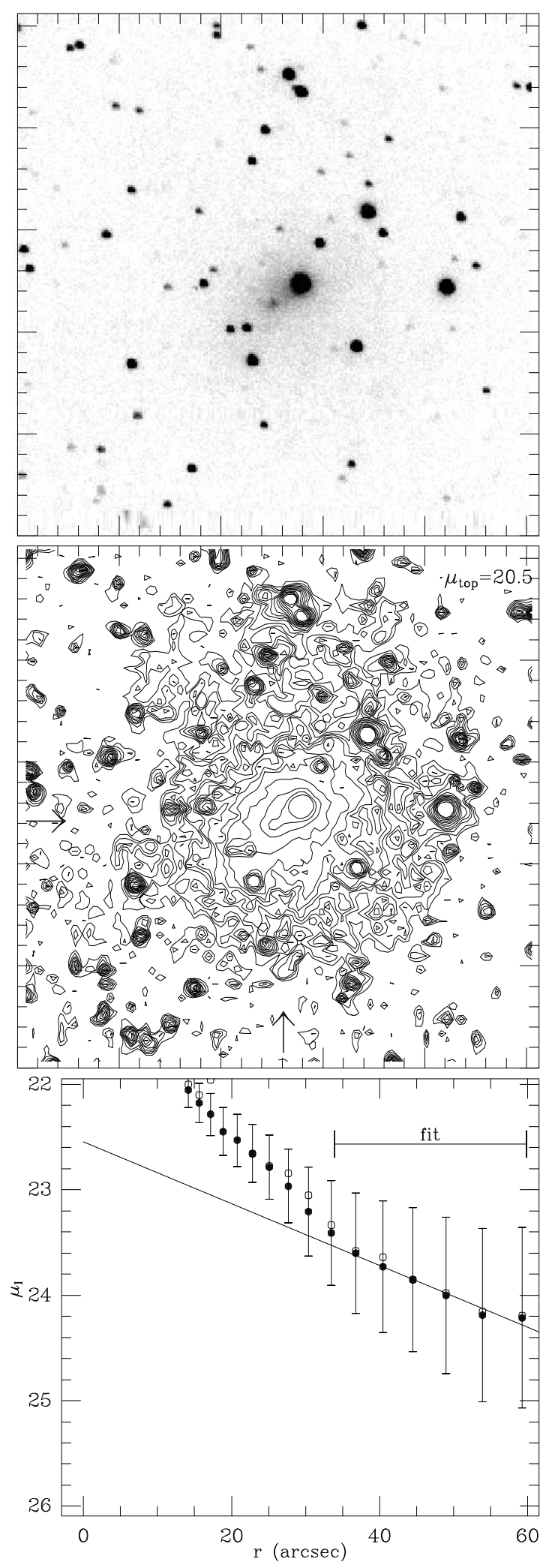

Figure 17: Karachentsev 37C (Sep91) 
B
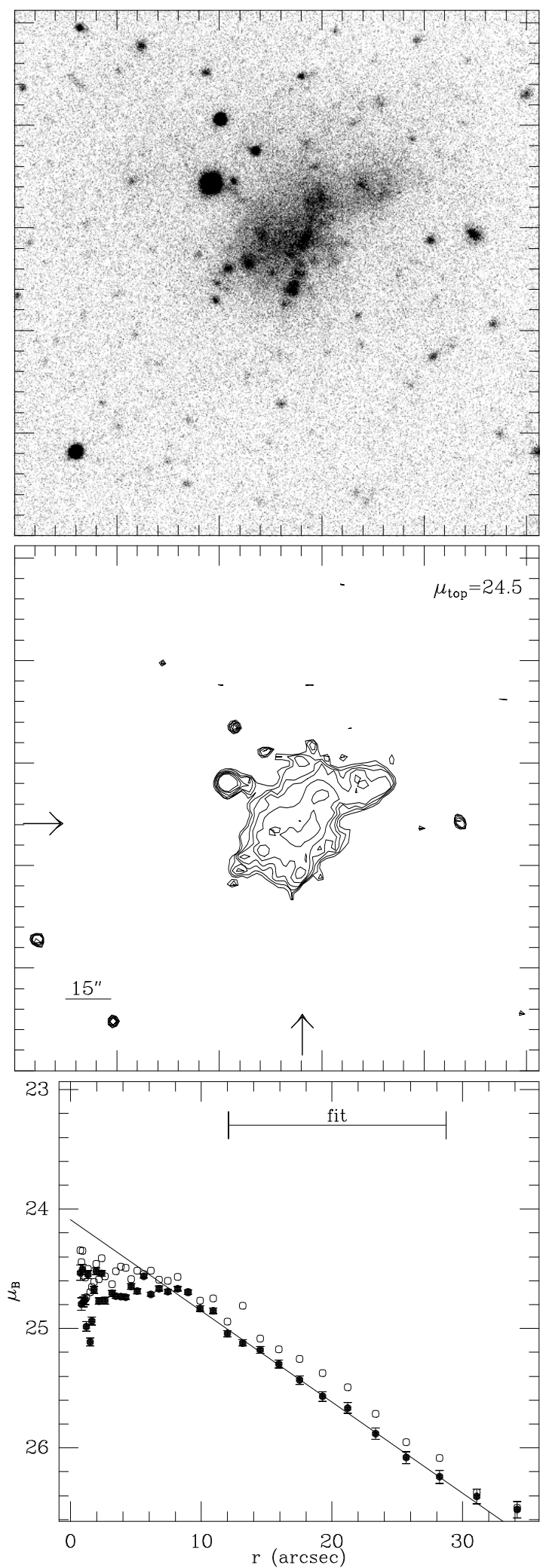

I

57
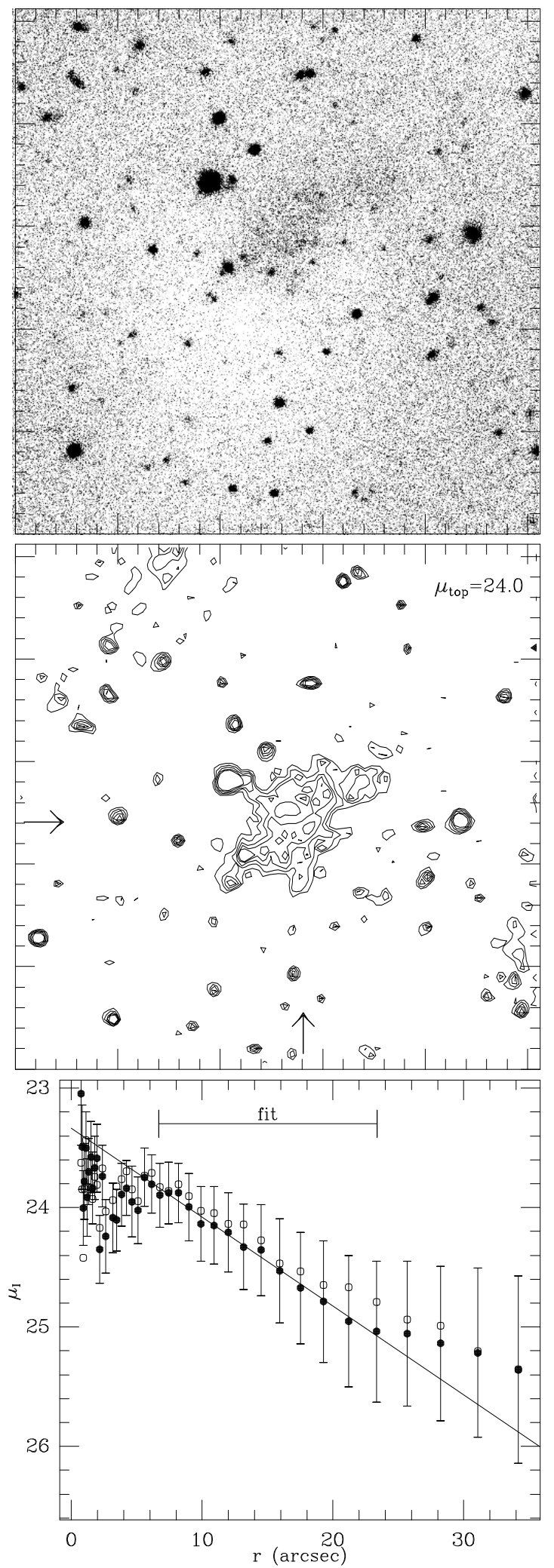

Figure 18: UGC 02162 
B
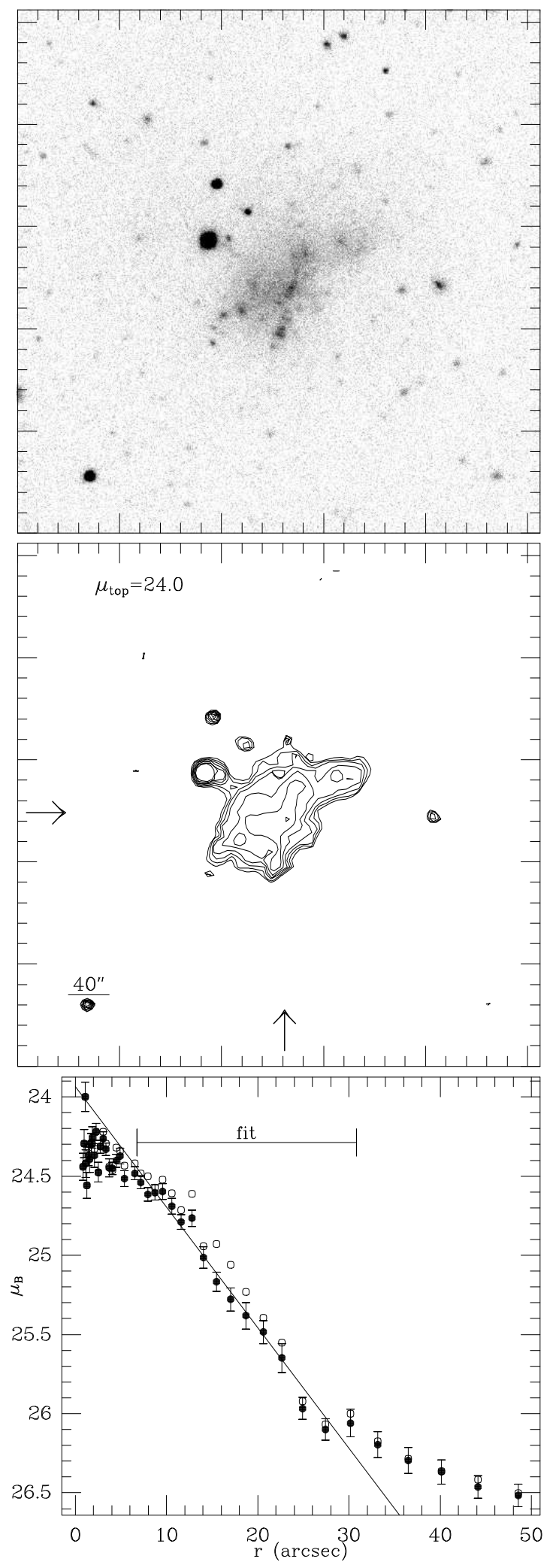

I
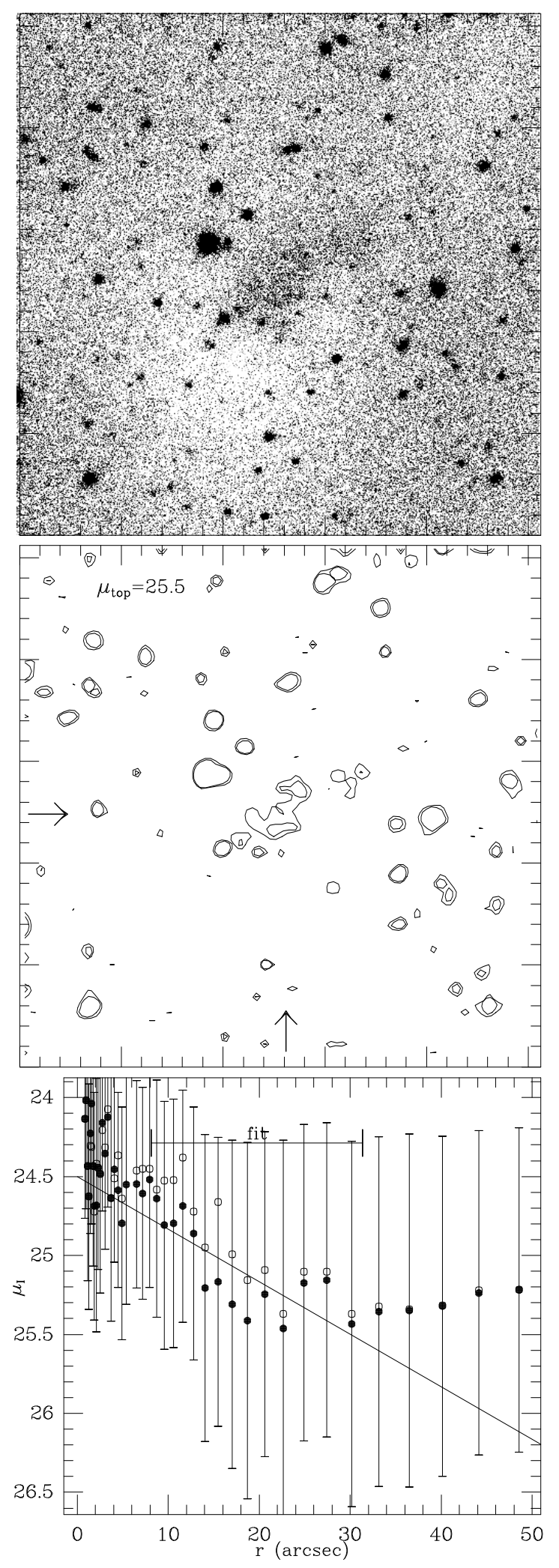

Figure 19: UGC 02162 (Jan93) 
B

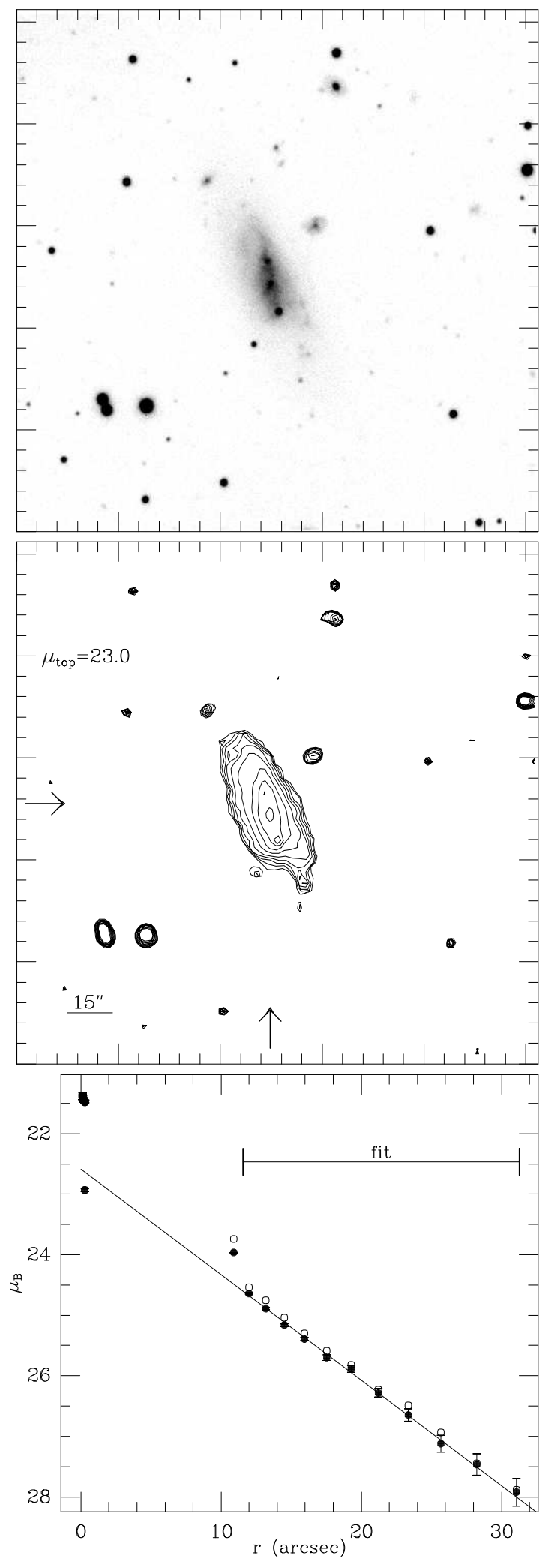

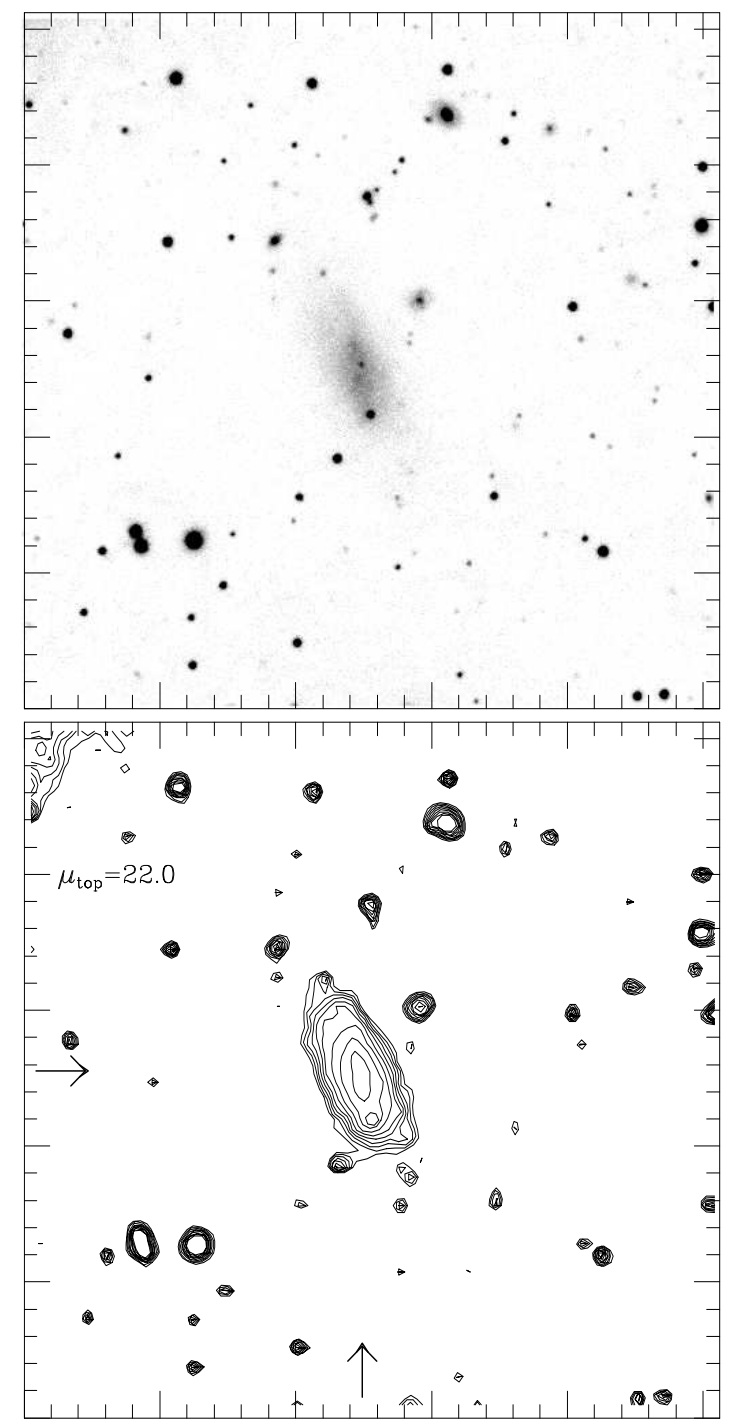

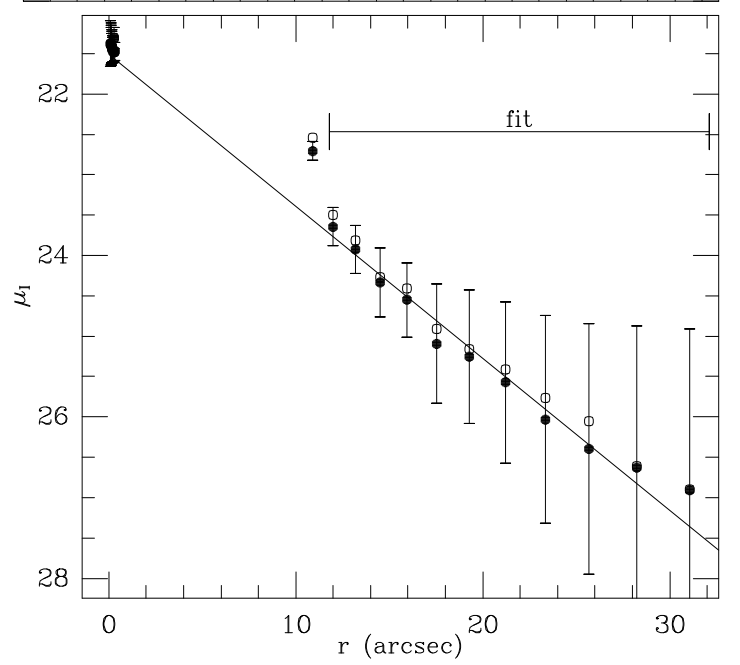

Figure 20: UGC 03212 
B

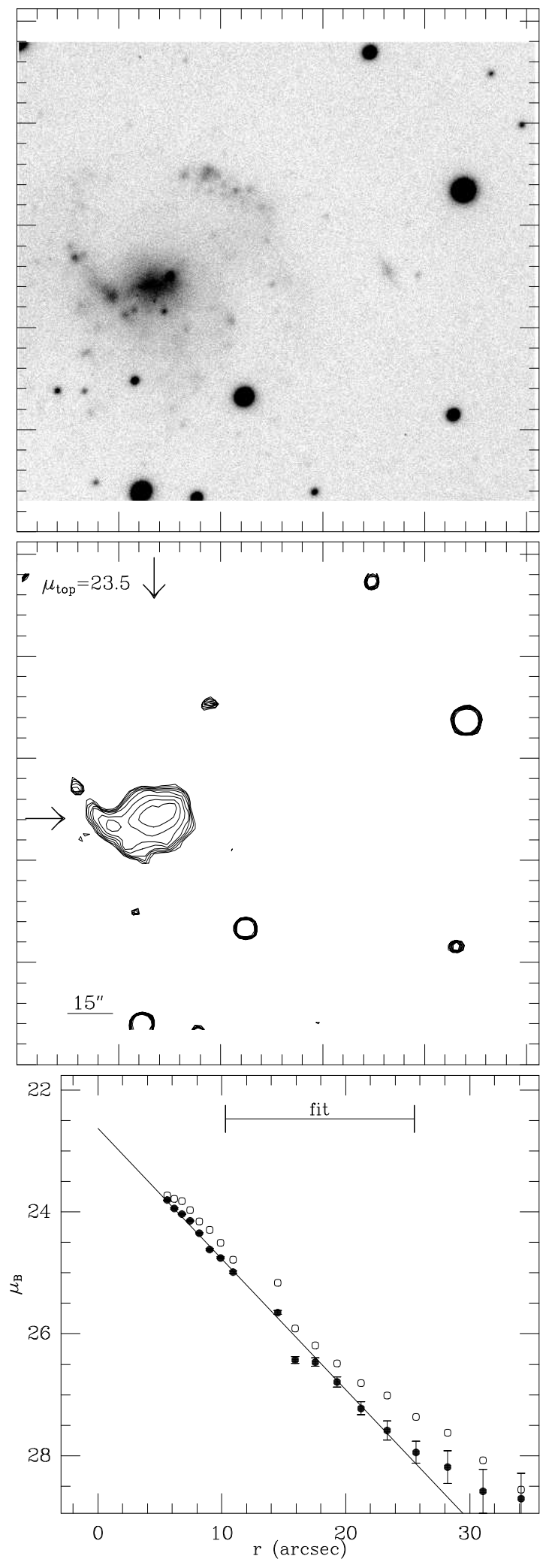

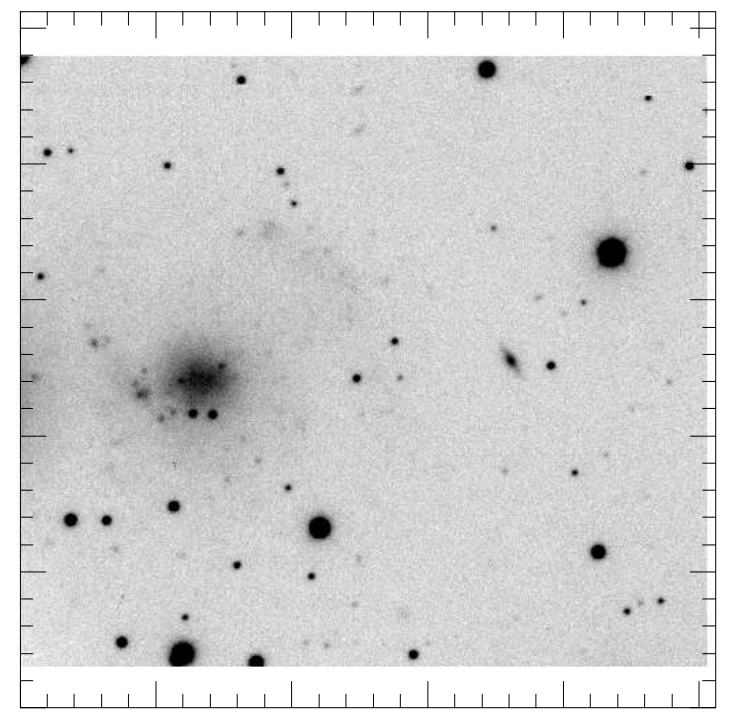
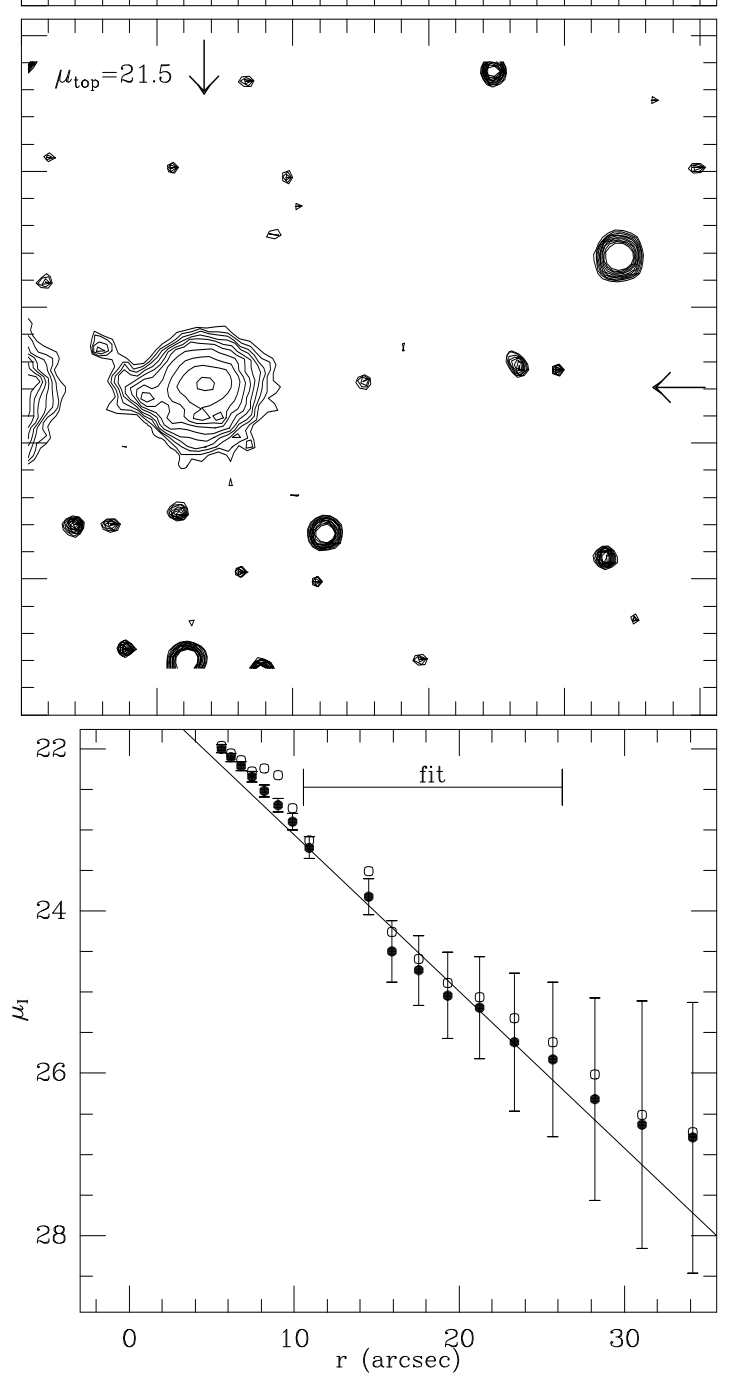

Figure 21: UGC 03384 
B

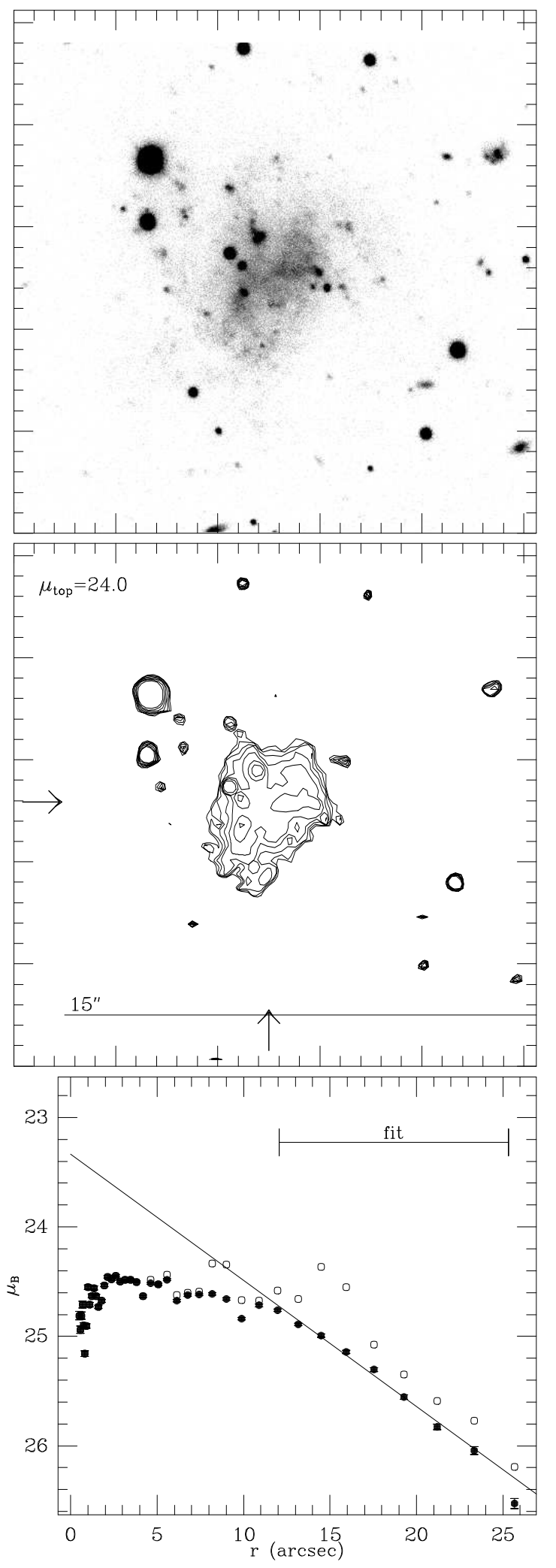

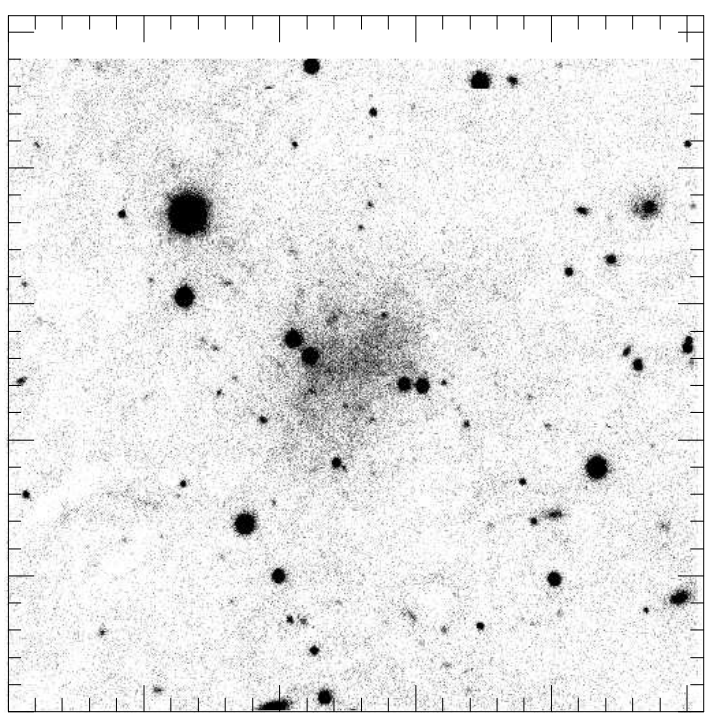
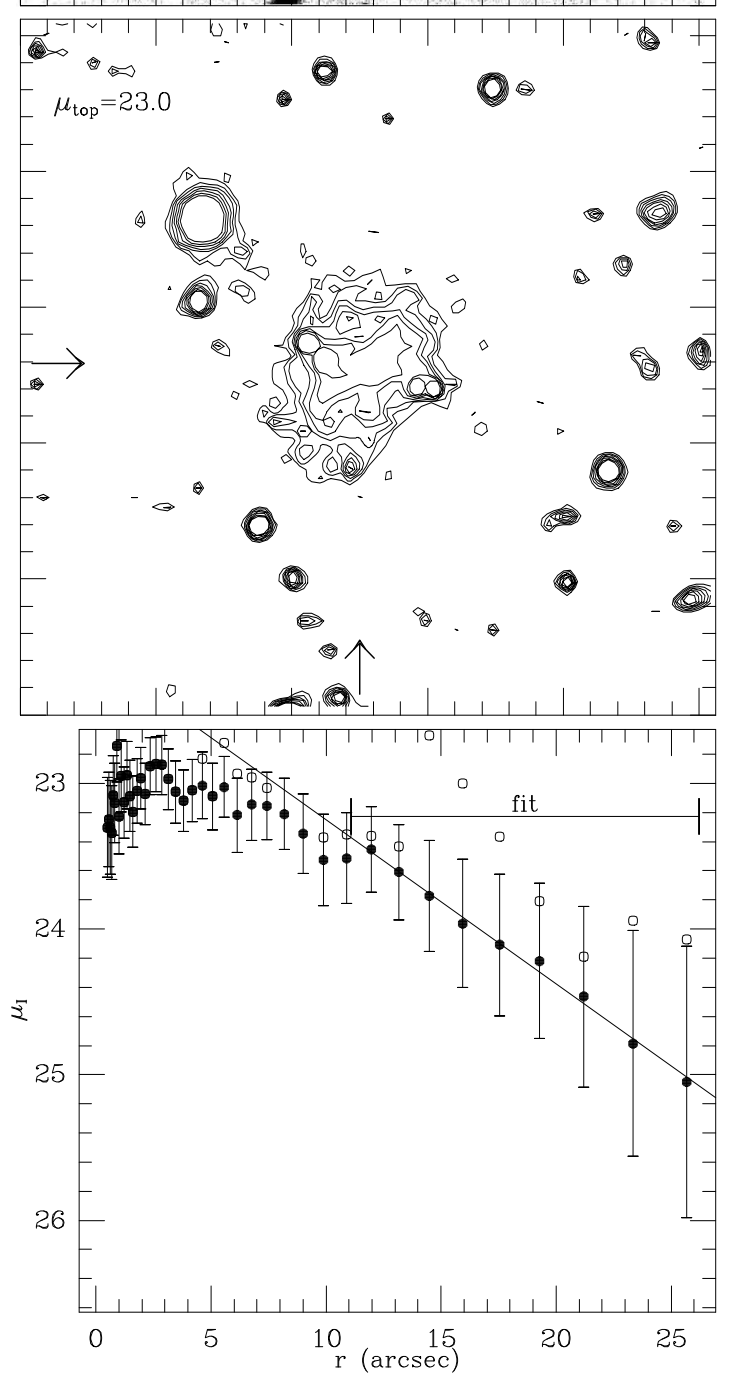

Figure 22: UGC 03817 
B

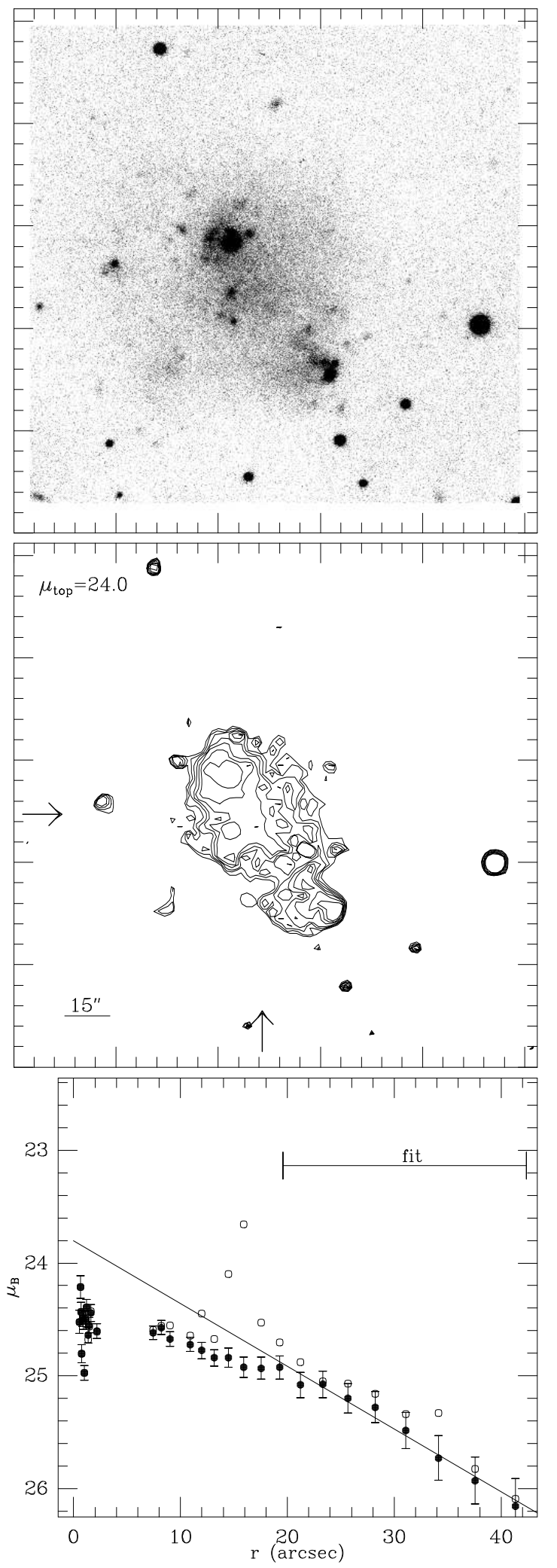

I

62
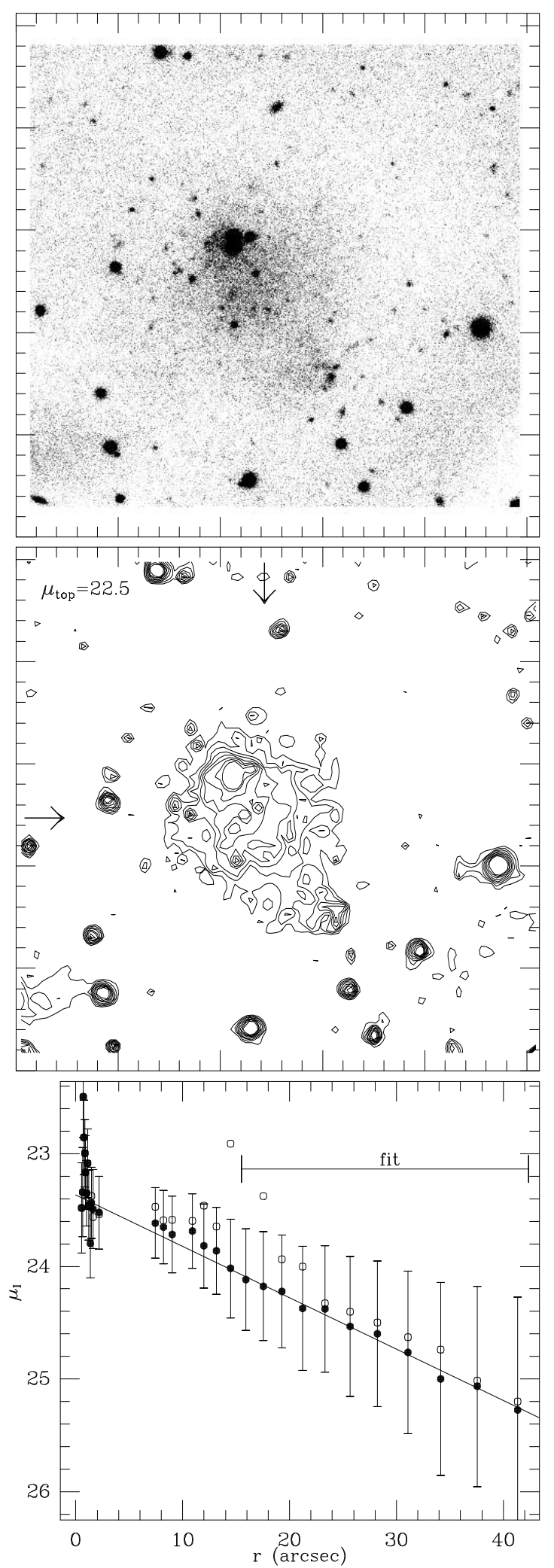

Figure 23: UGC 03966 
B

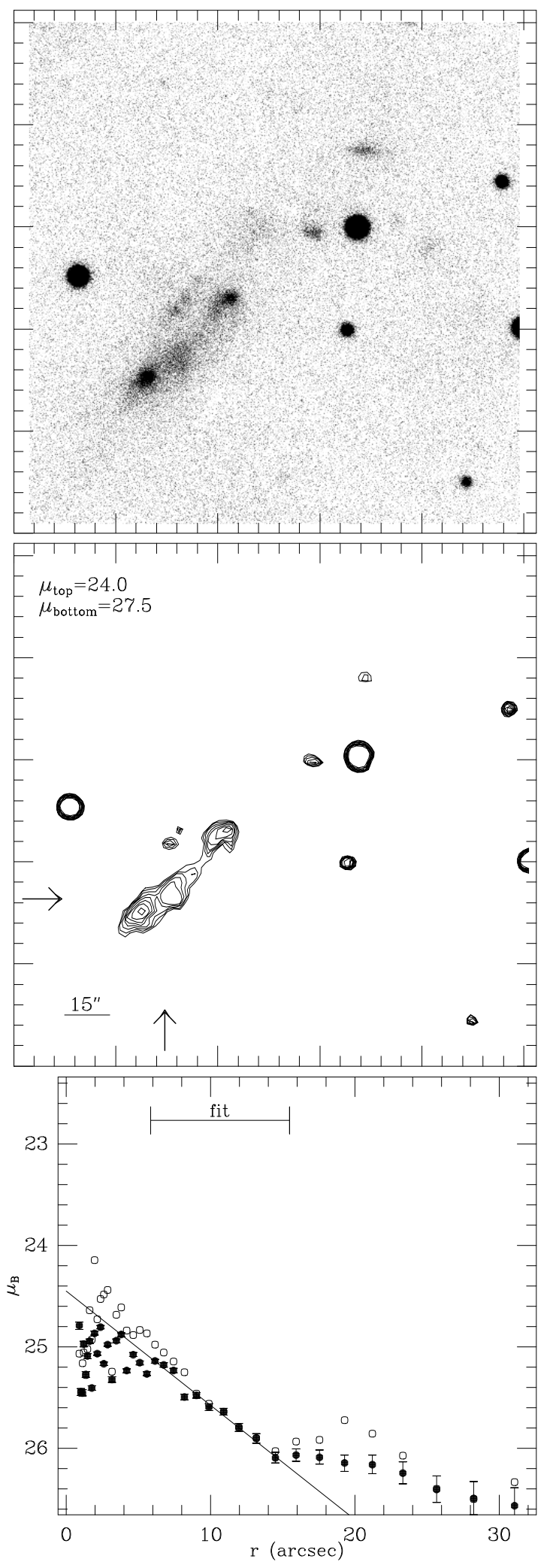

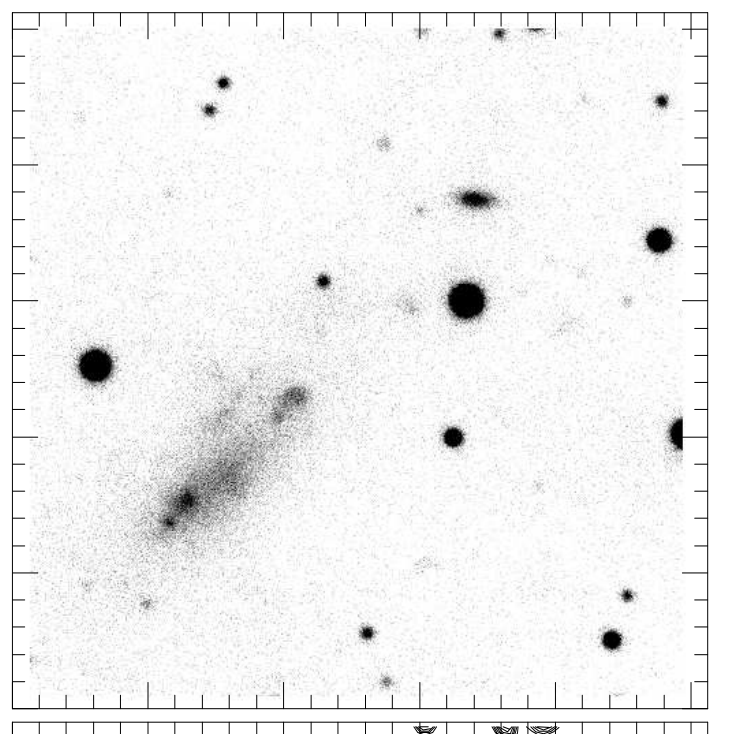
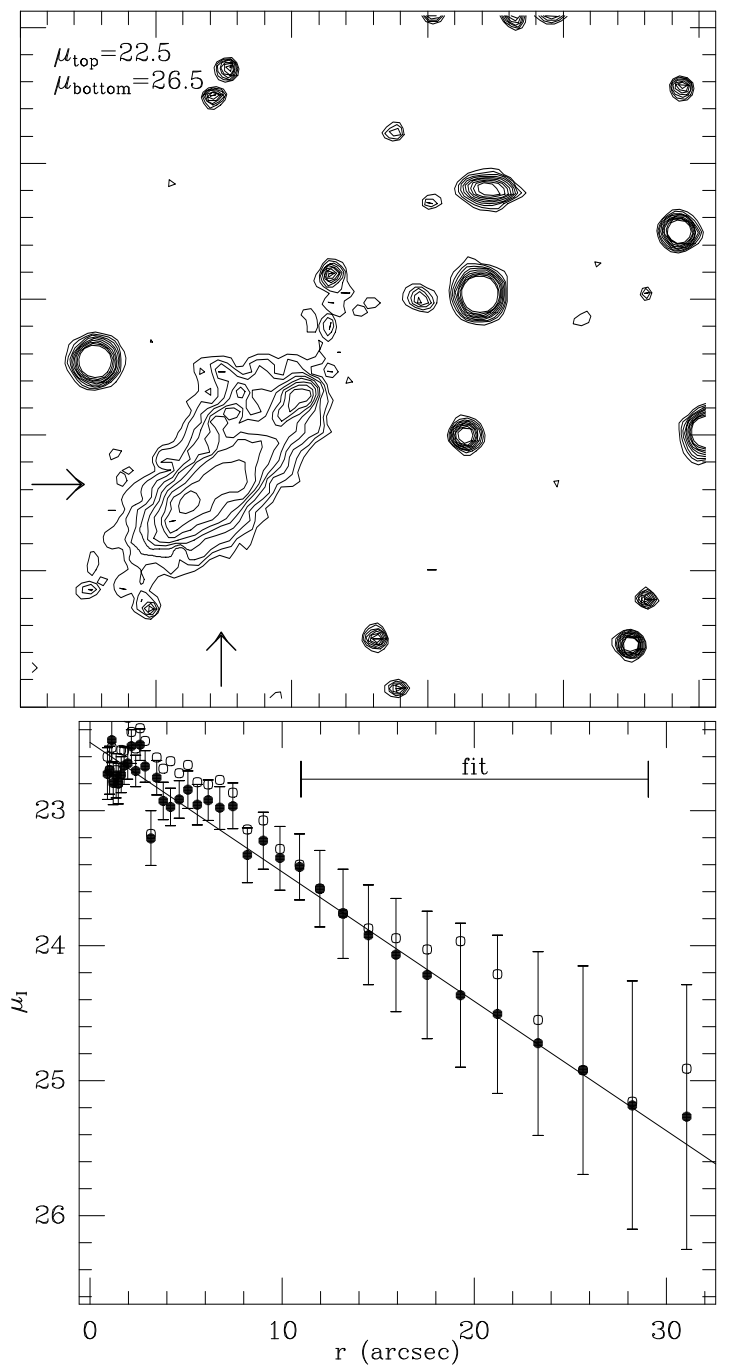

Figure 24: UGC 04173 

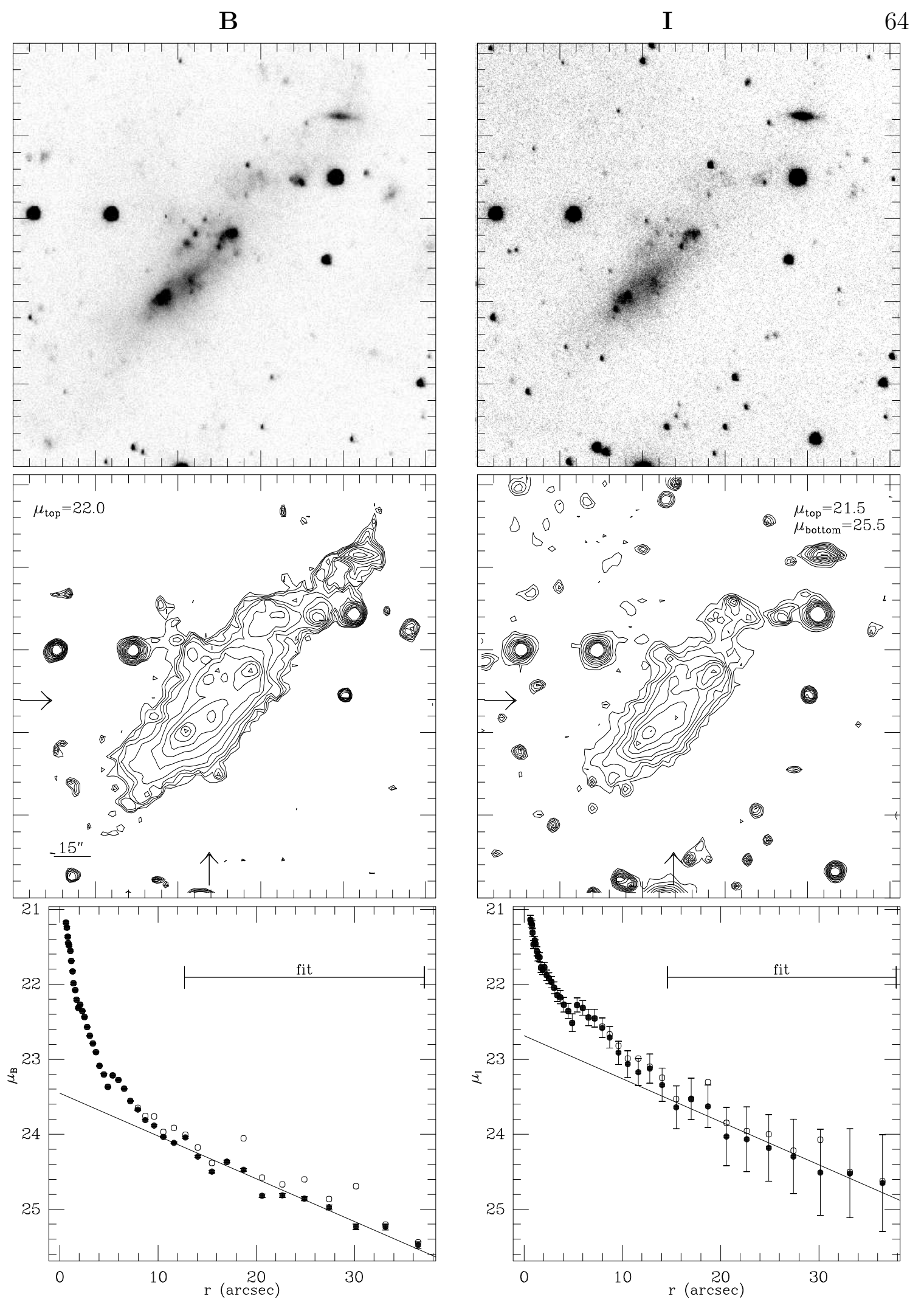

Figure 25: UGC 04173 (Jan93) 
B

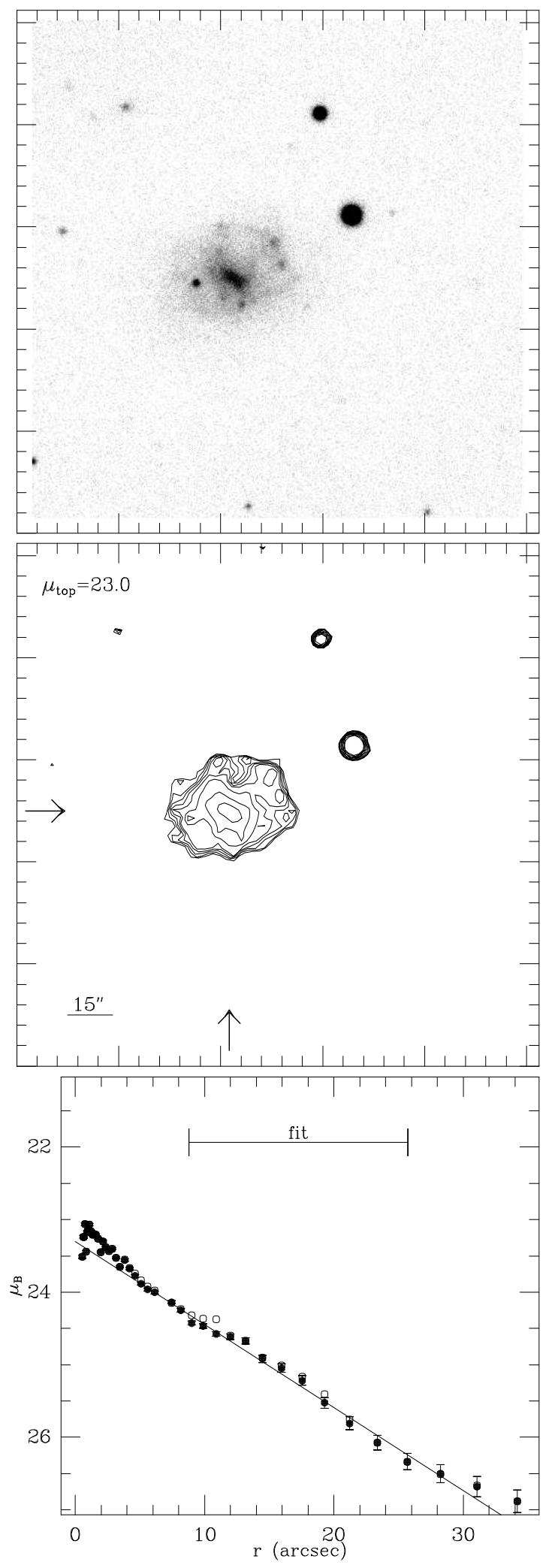

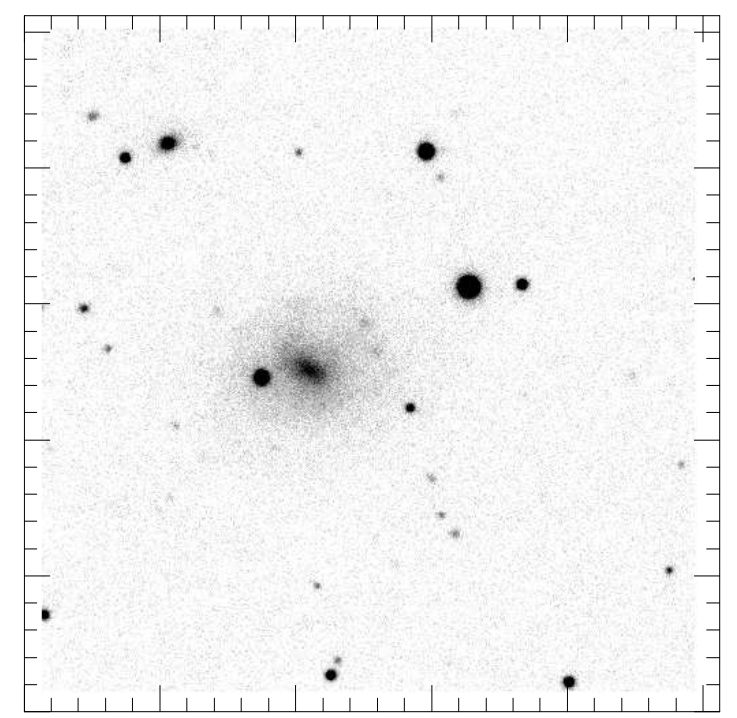
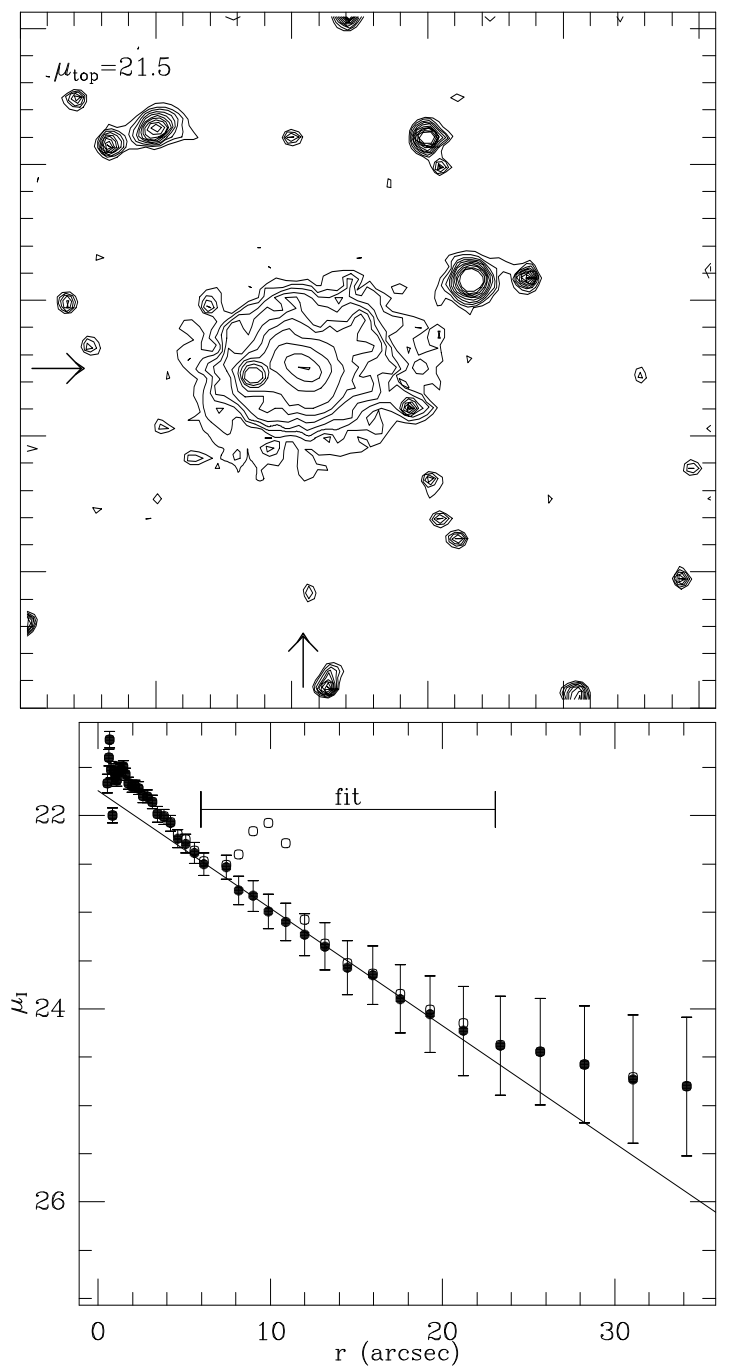

Figure 26: UGC 04204 
B
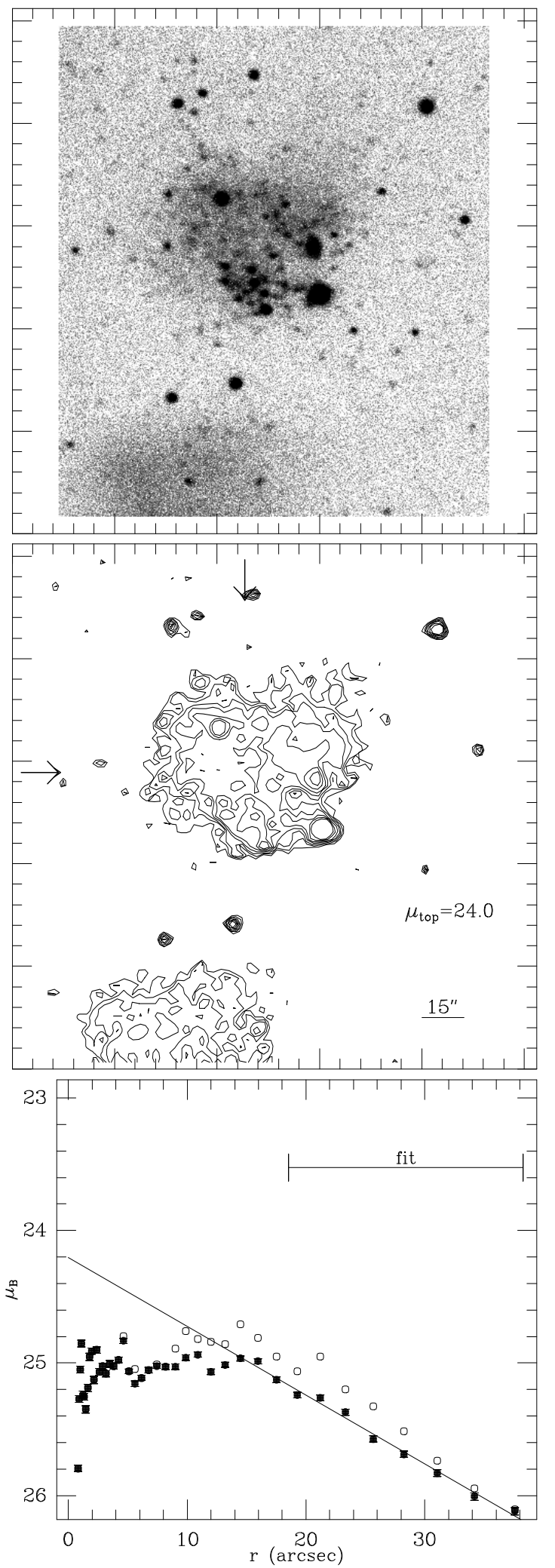

I

66
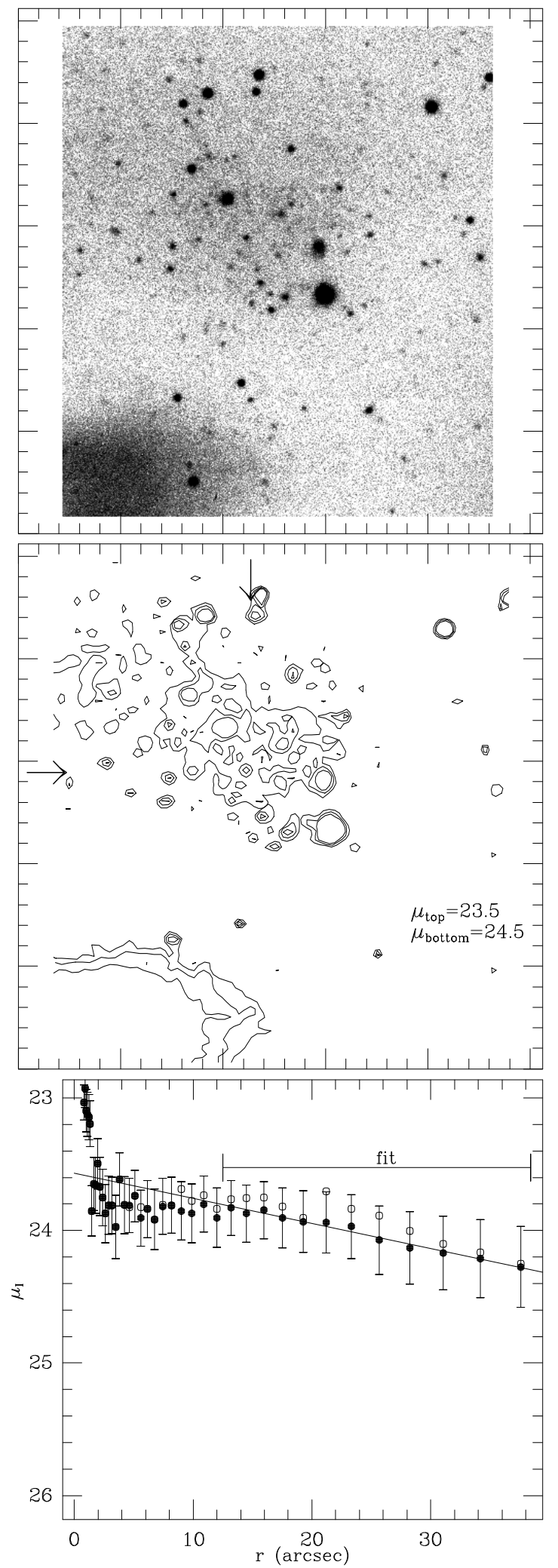

Figure 27: M81dwA 
B
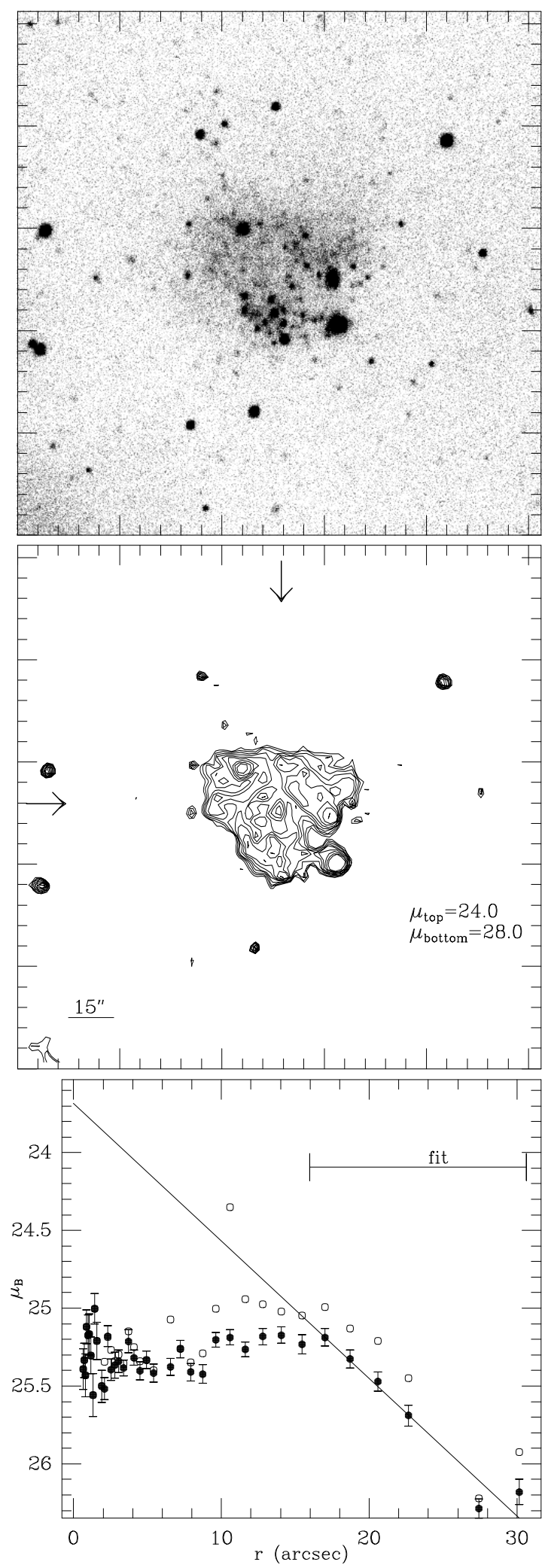

I

67
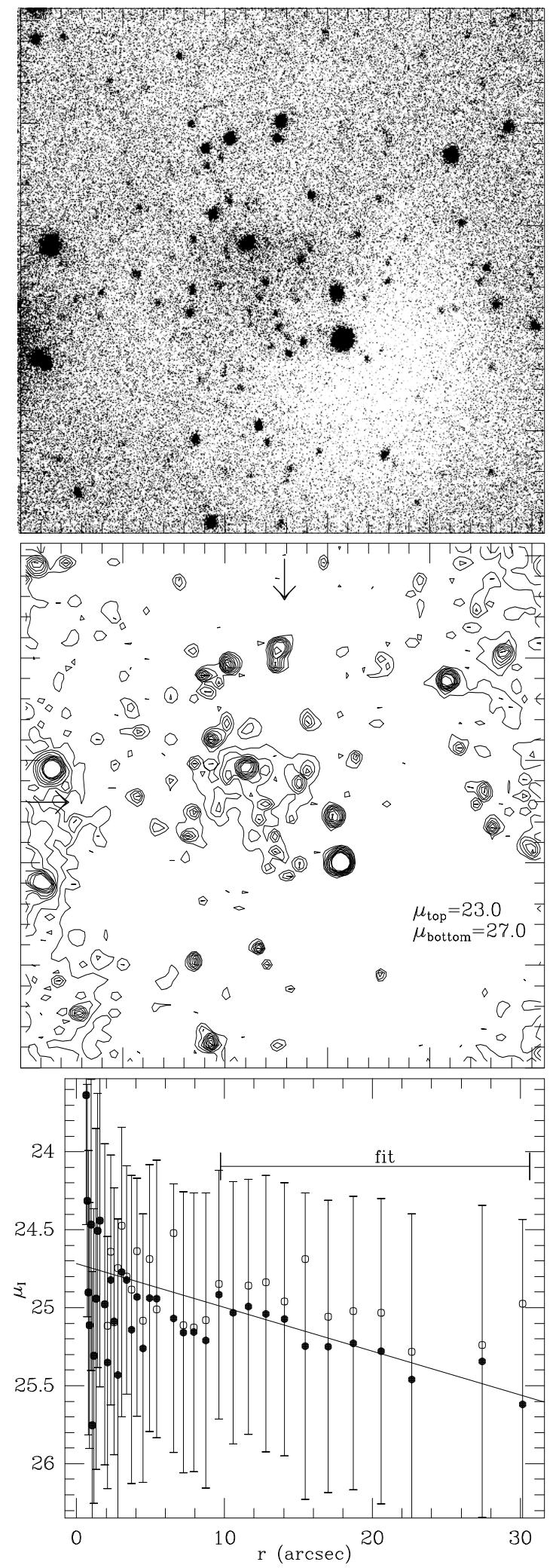

Figure 28: M81dwA (Jan93) 
B

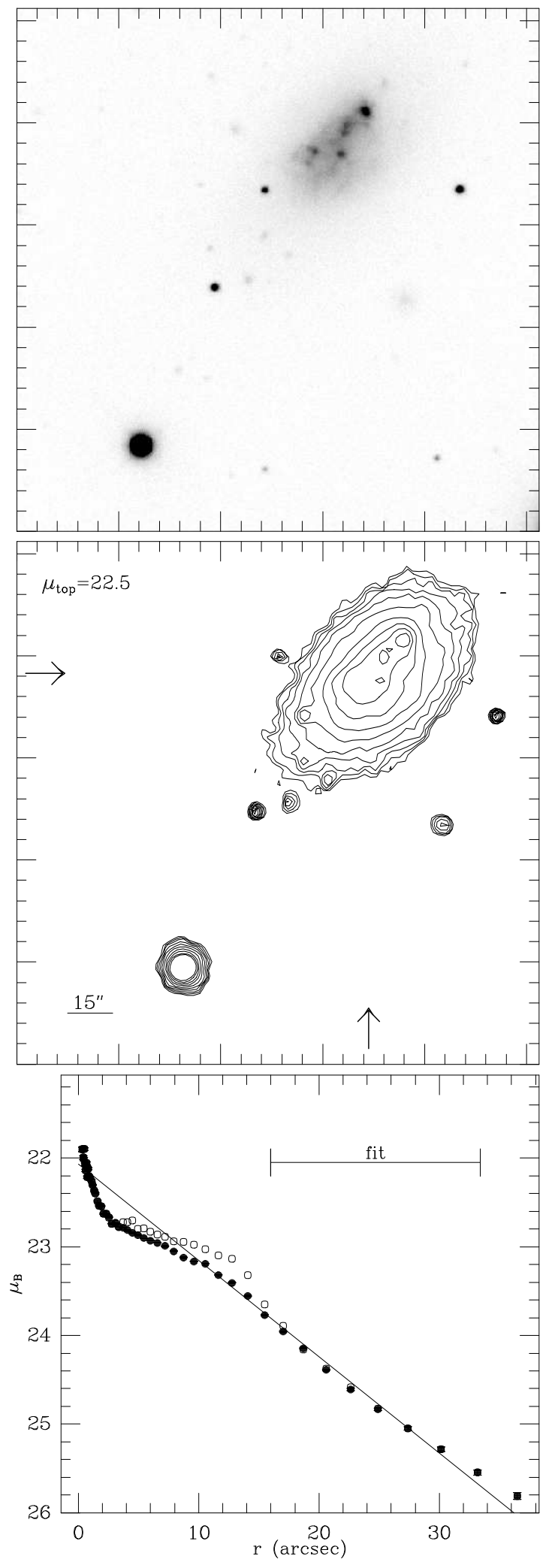

68
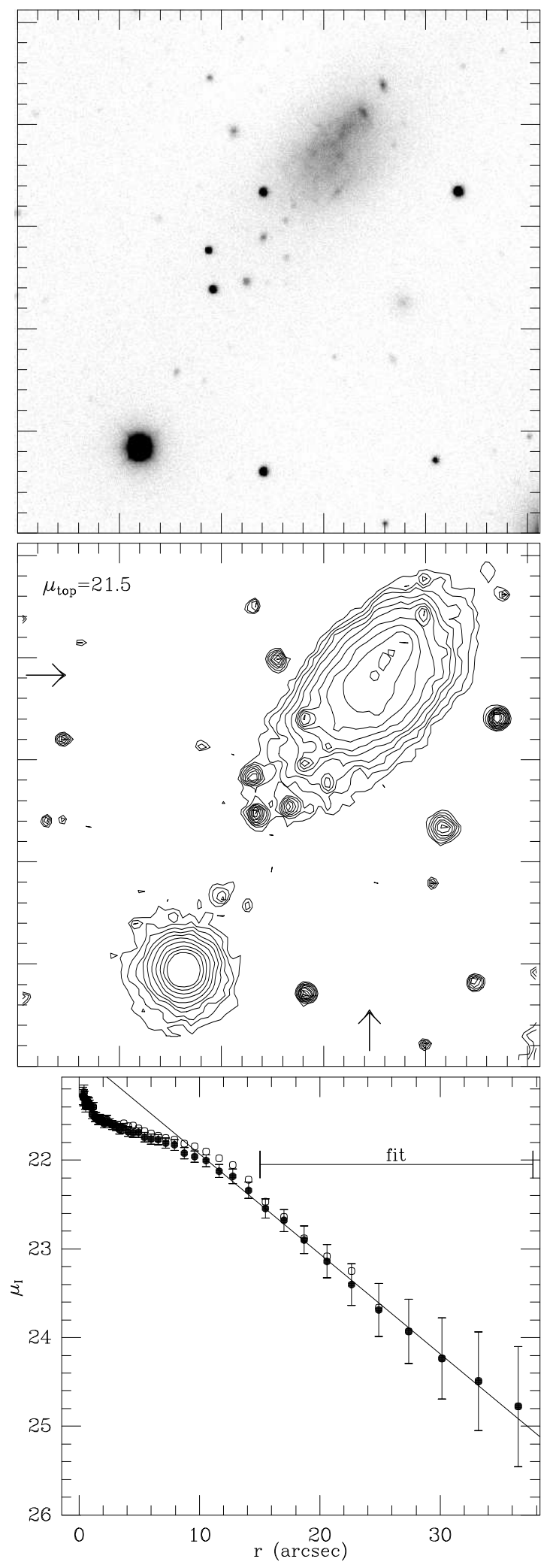

Figure 29: UGC 05423 (M81dwB; Jan93) 
B
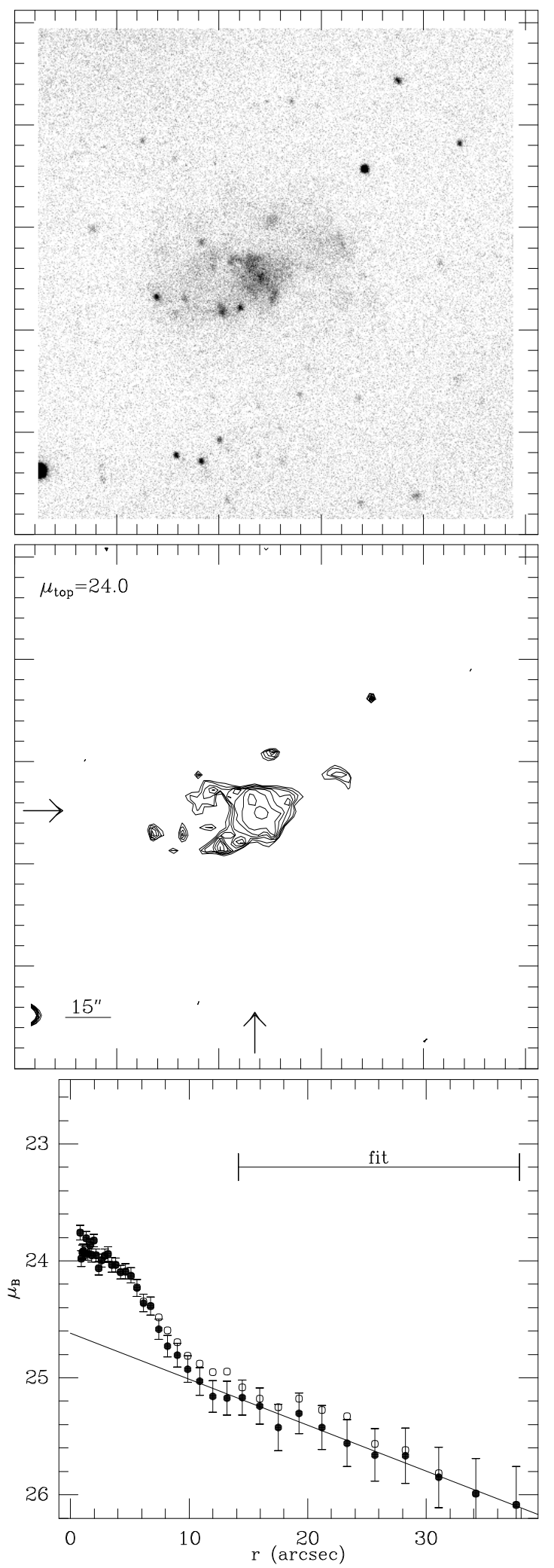

I

69
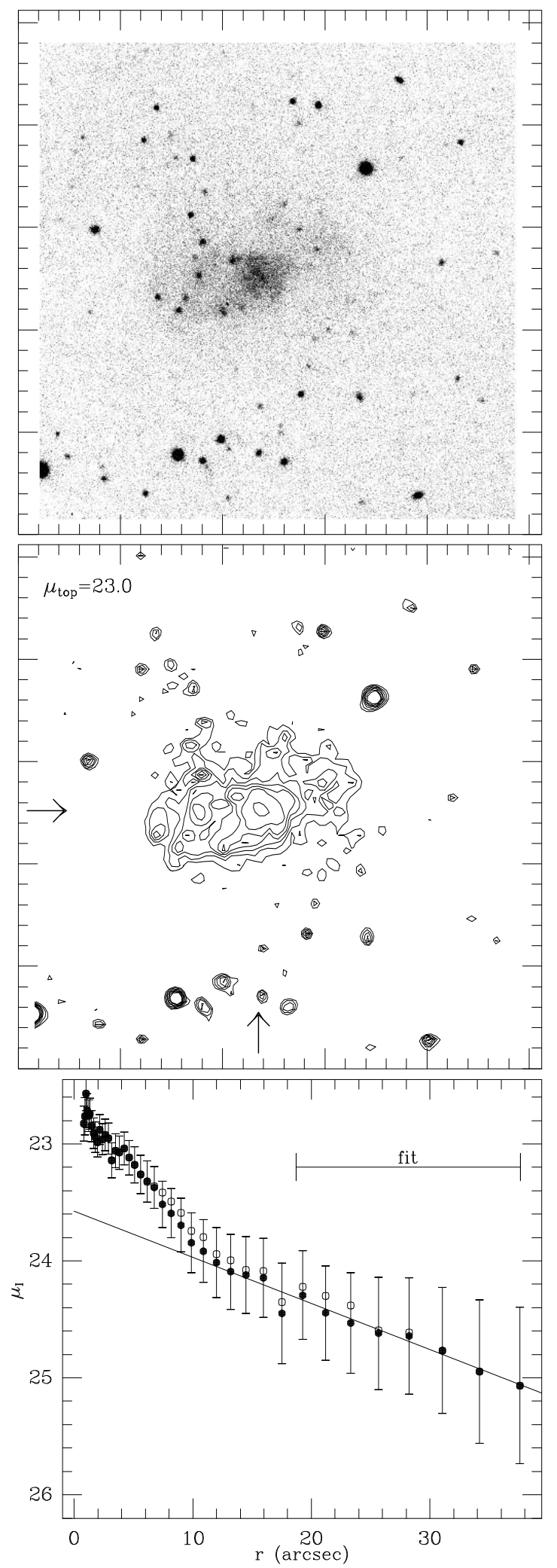

Figure 30: UGC 05706 
B

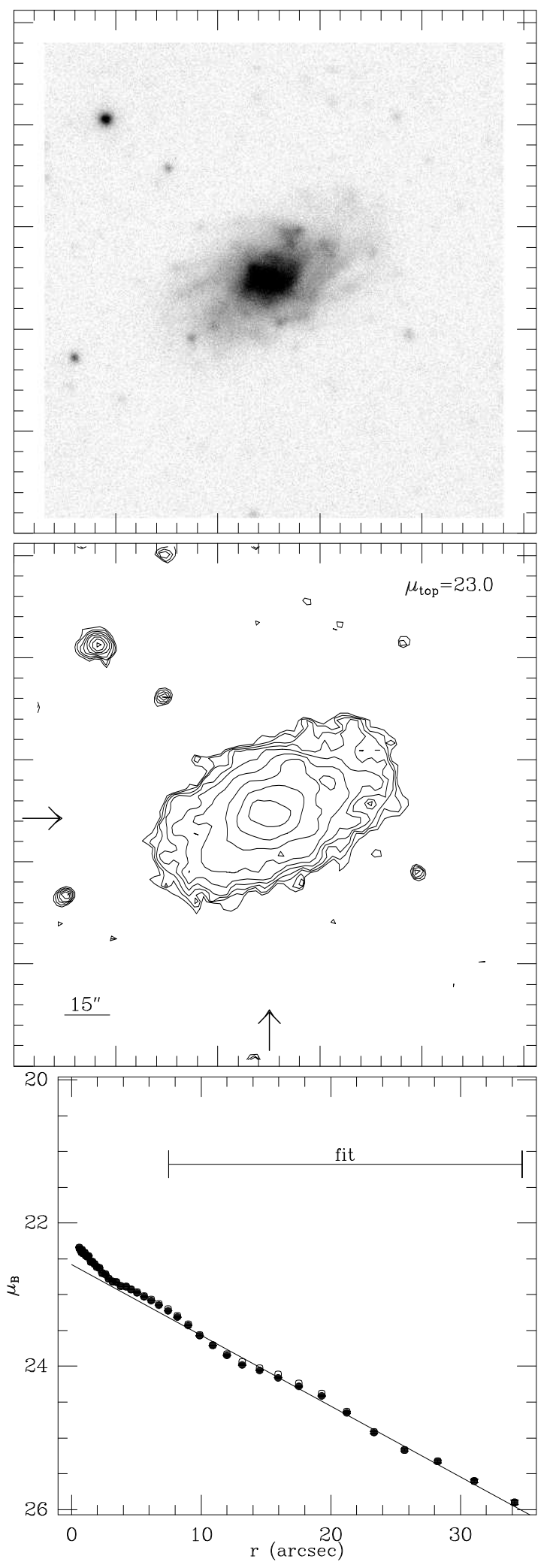

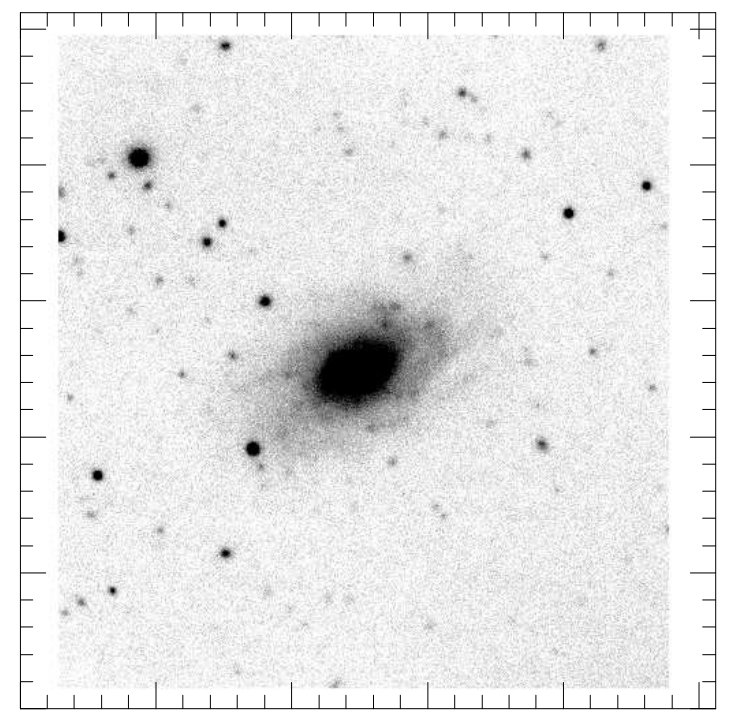
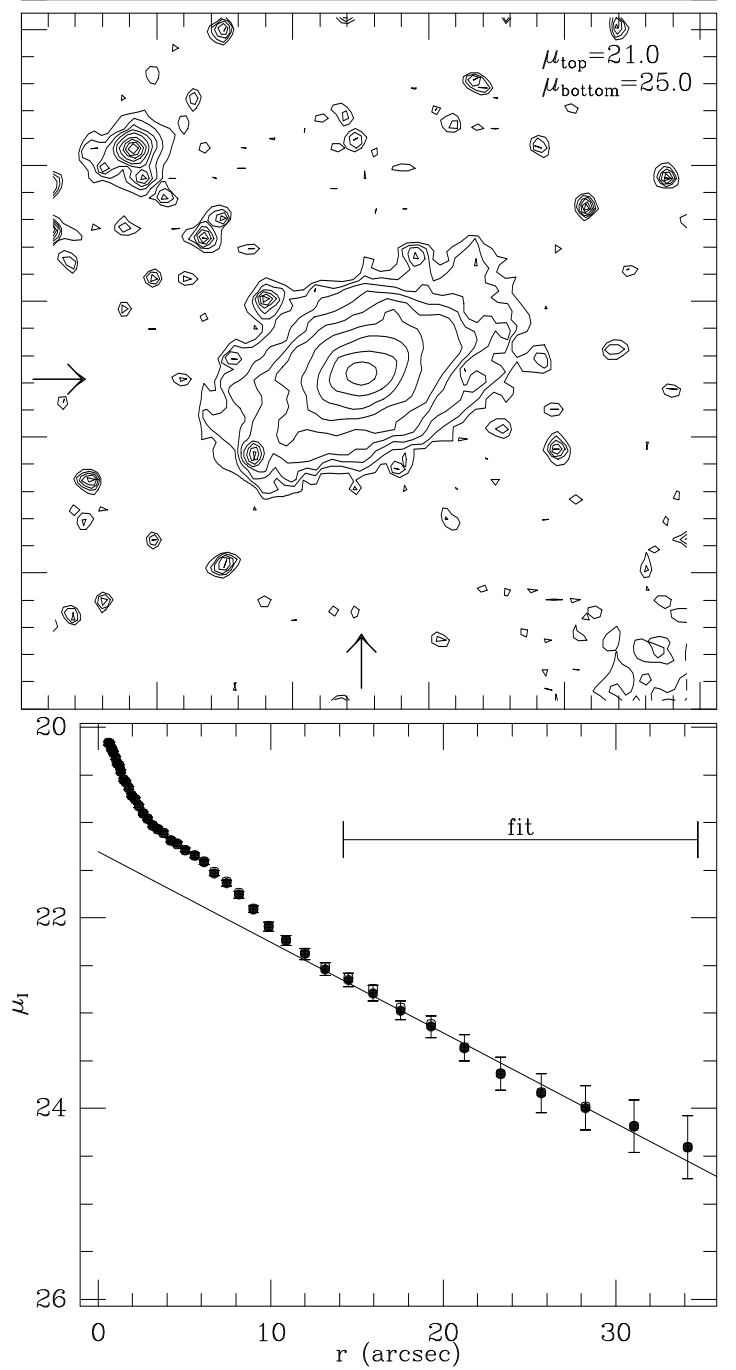

Figure 31: UGC 05709 
B

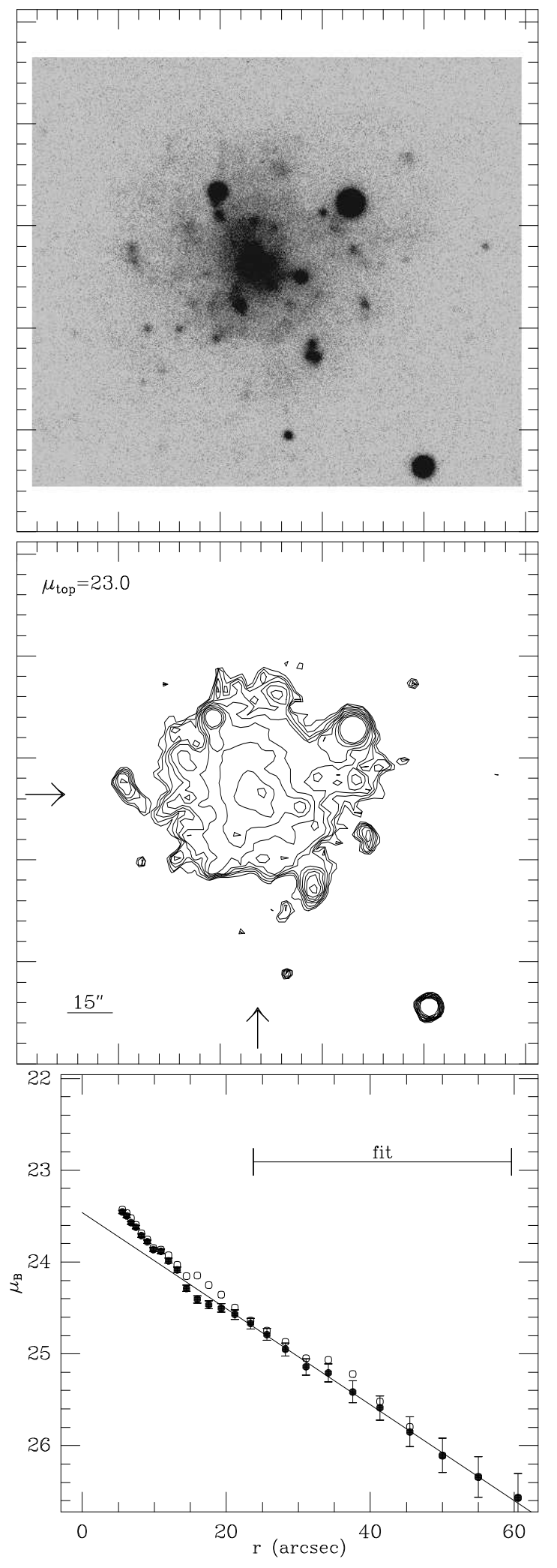

I
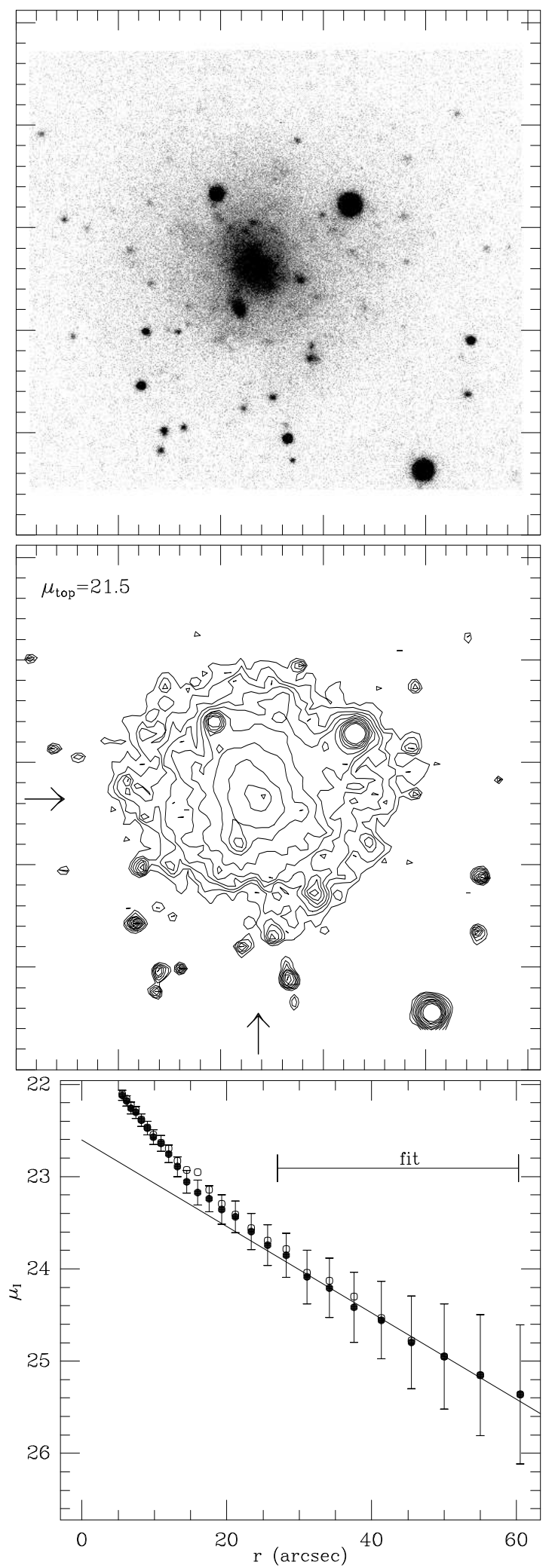

Figure 32: UGC 06151 
B

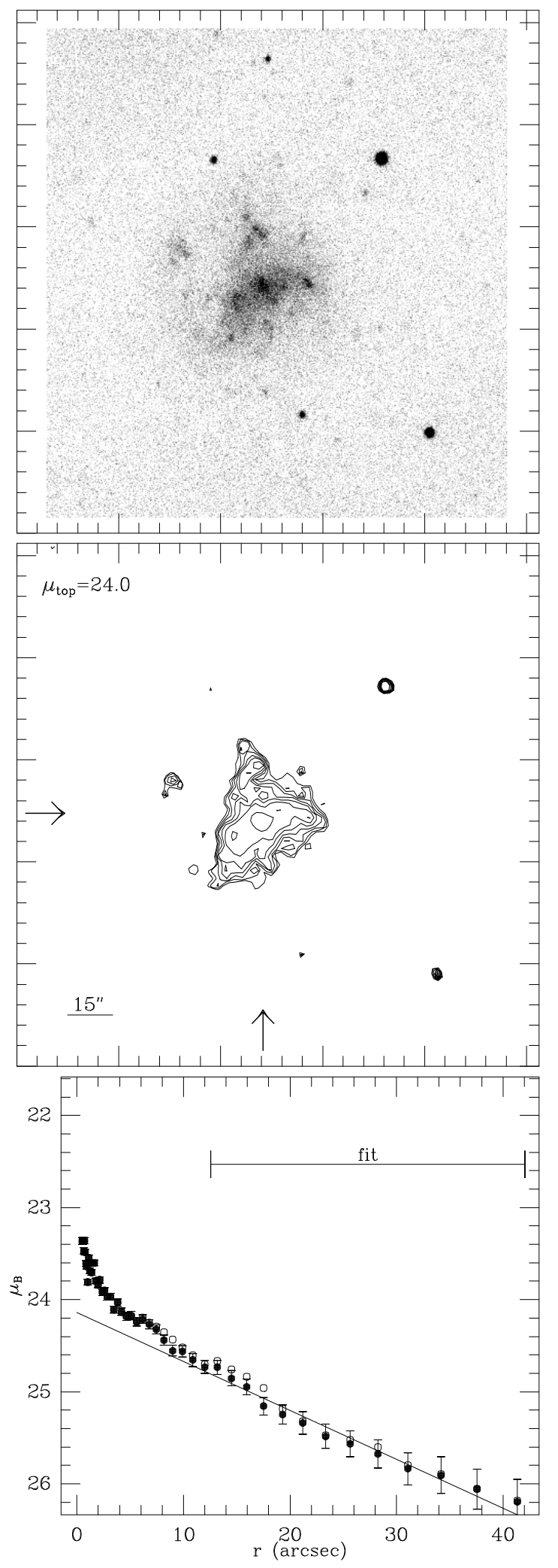

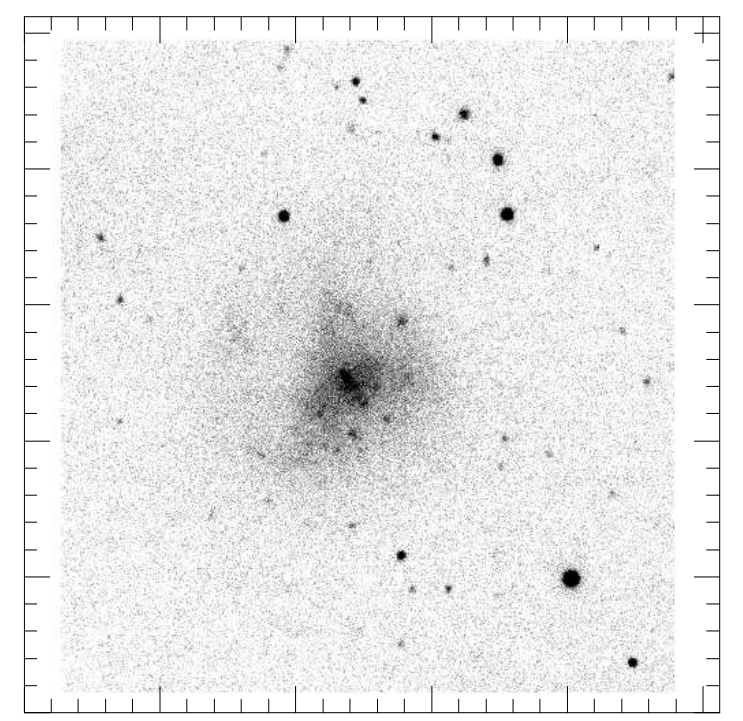
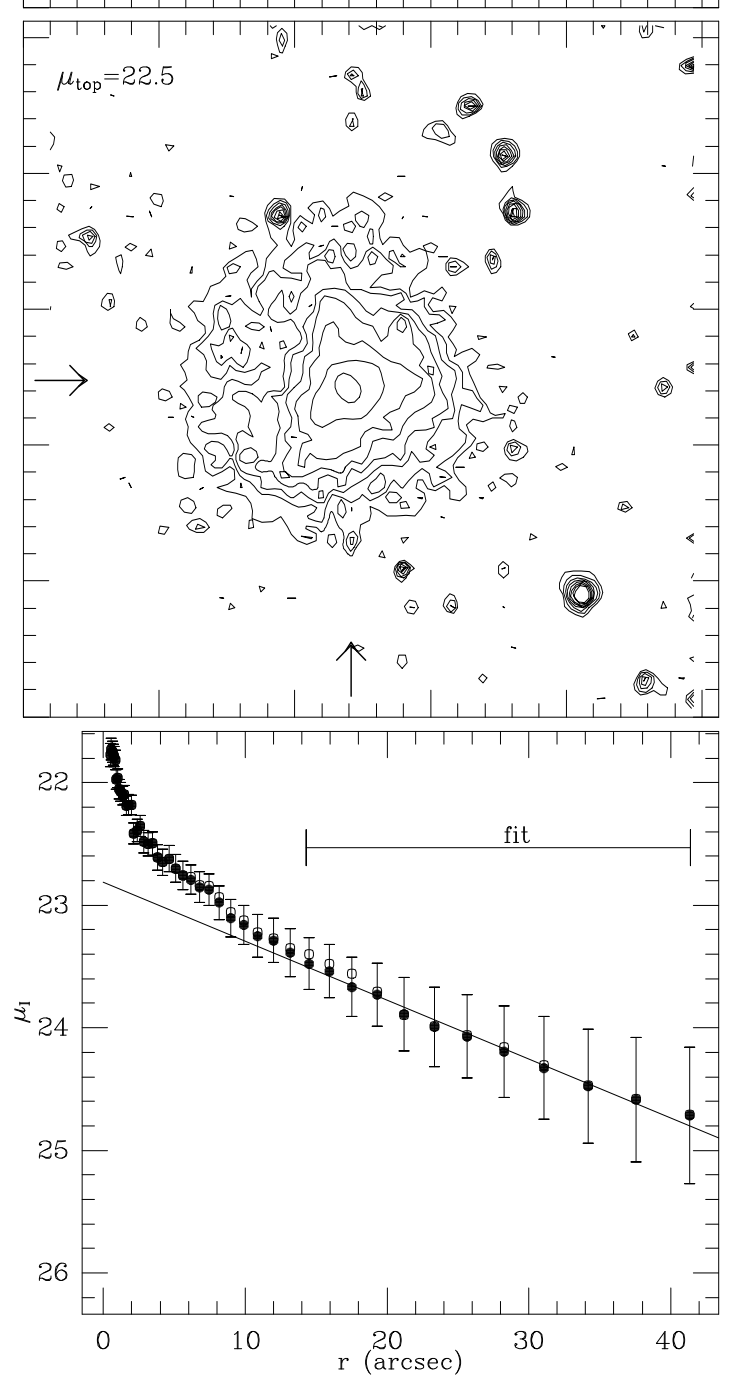

Figure 33: UGC 06248 
B
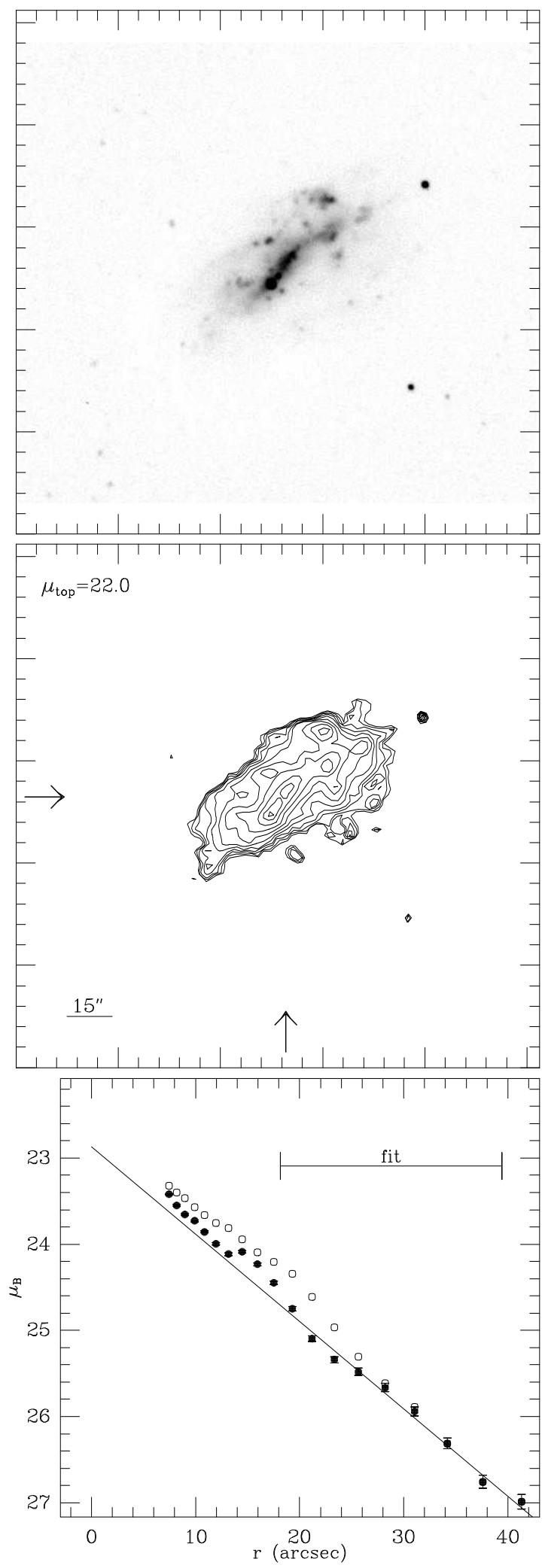

I

73
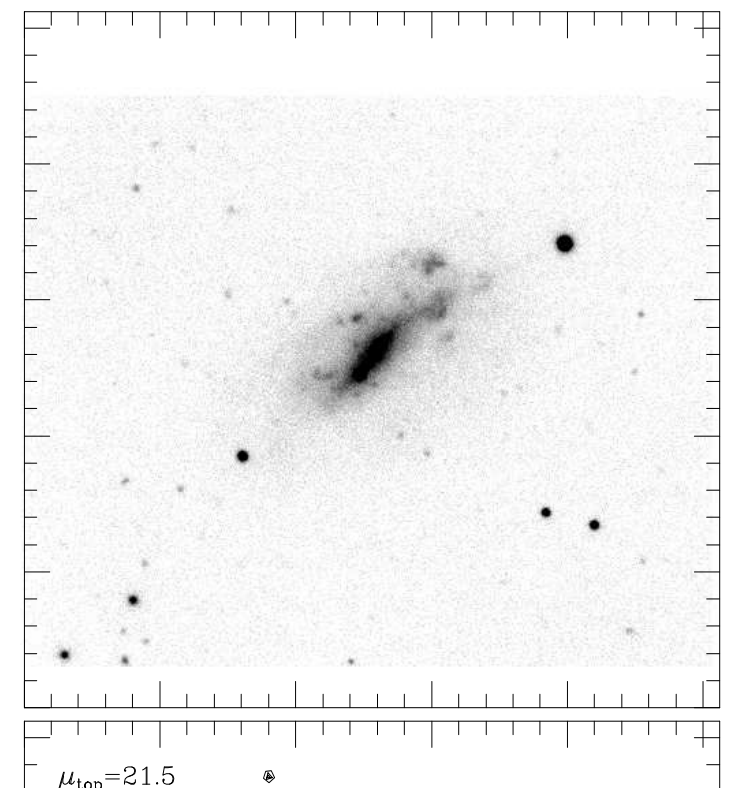

-
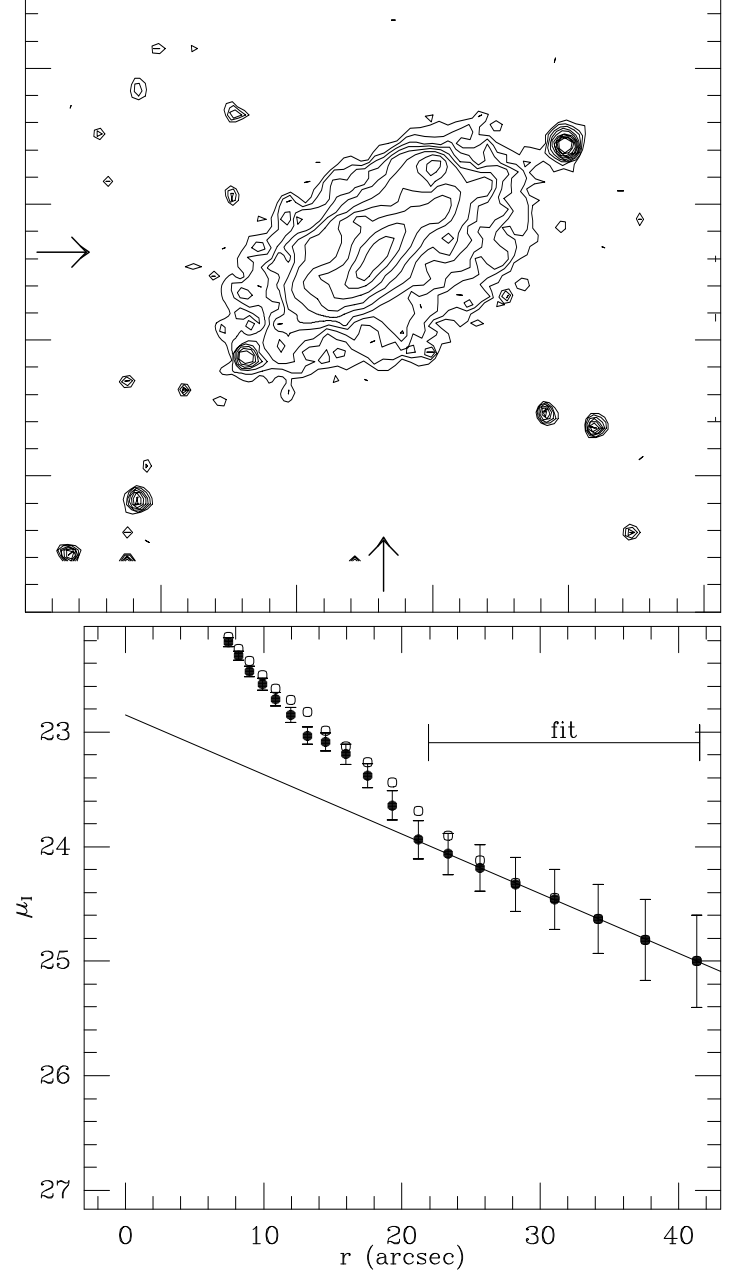

Figure 34: UGC 06596 
B

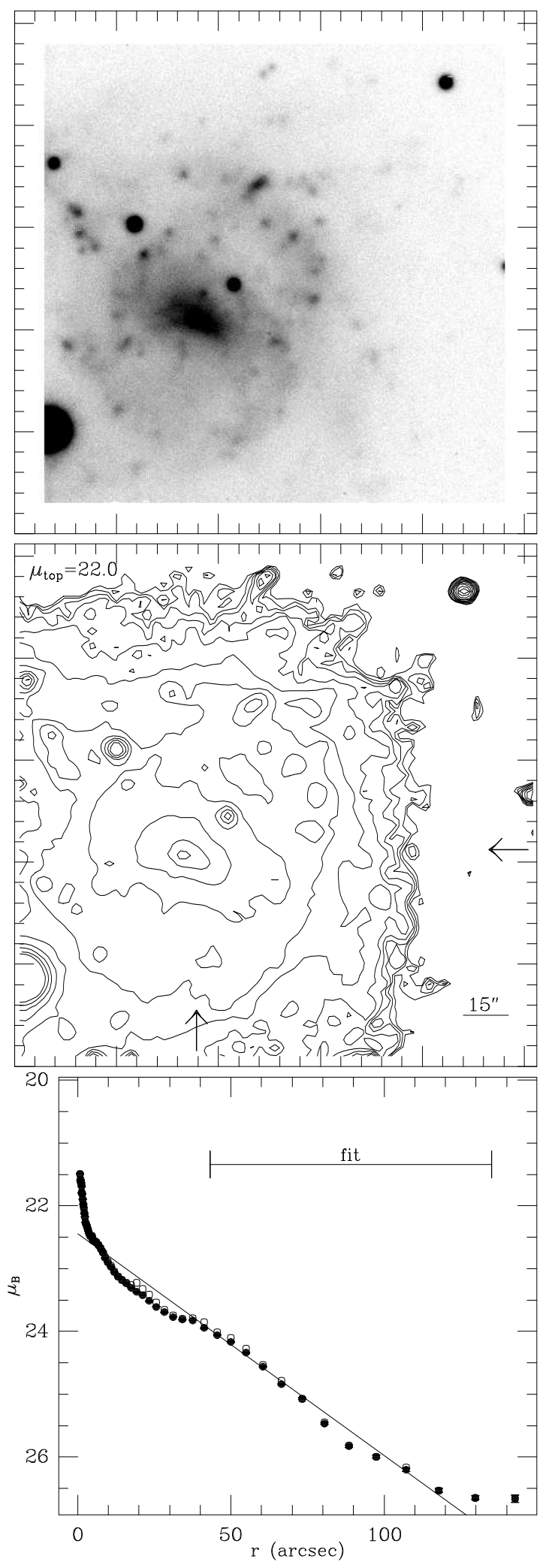

I

74
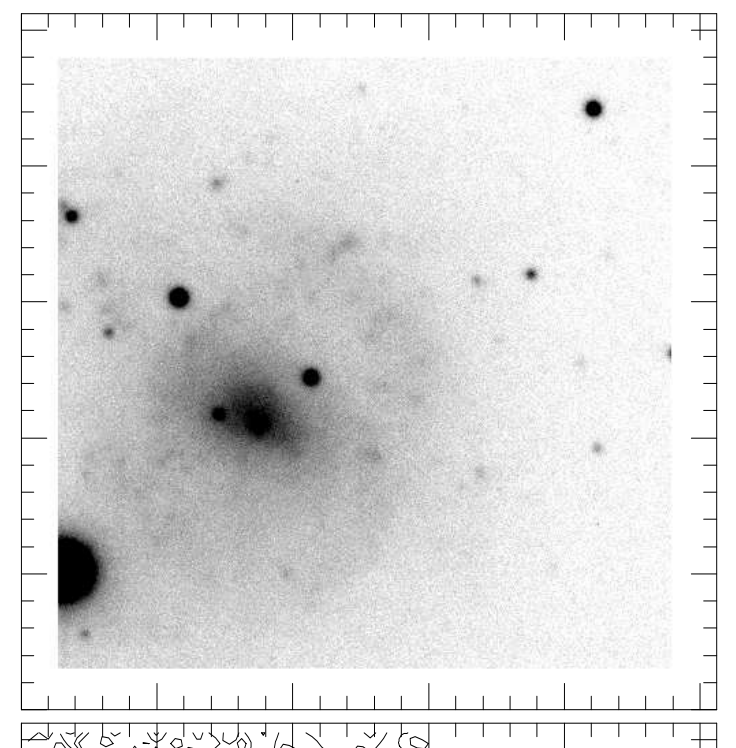

-2 200 a
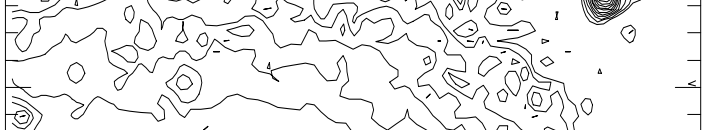

-
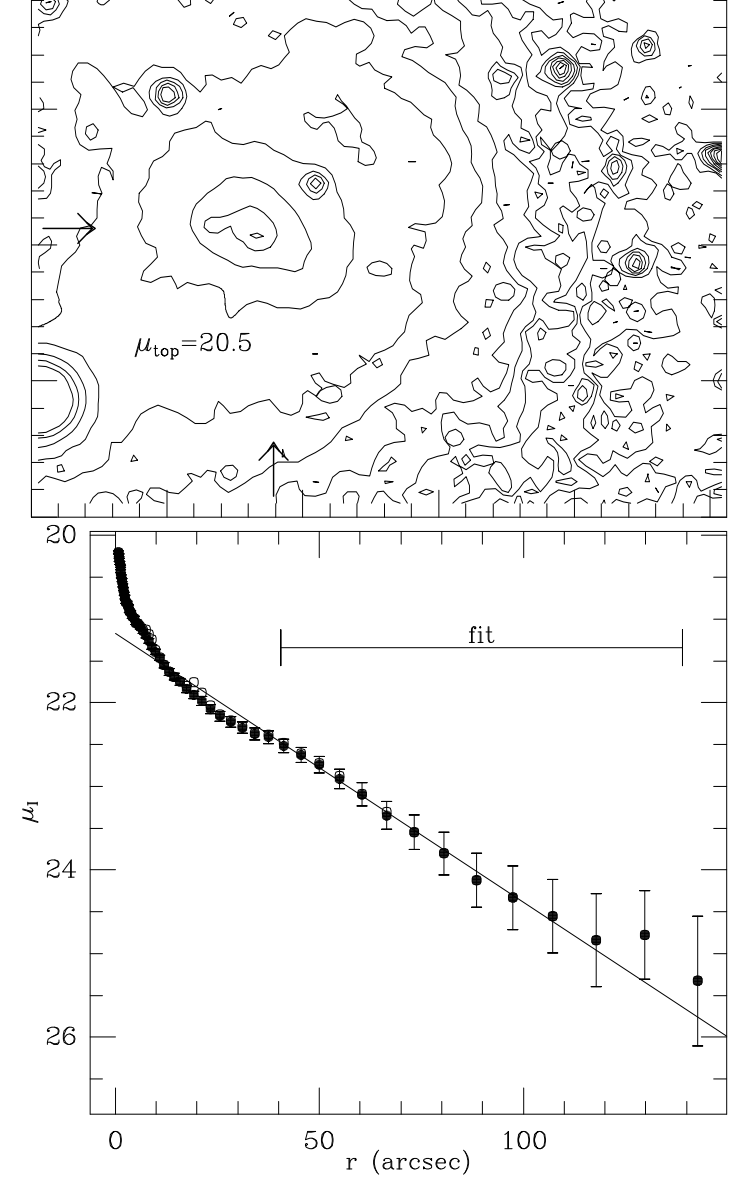

Figure 35: UGC 06628 
B

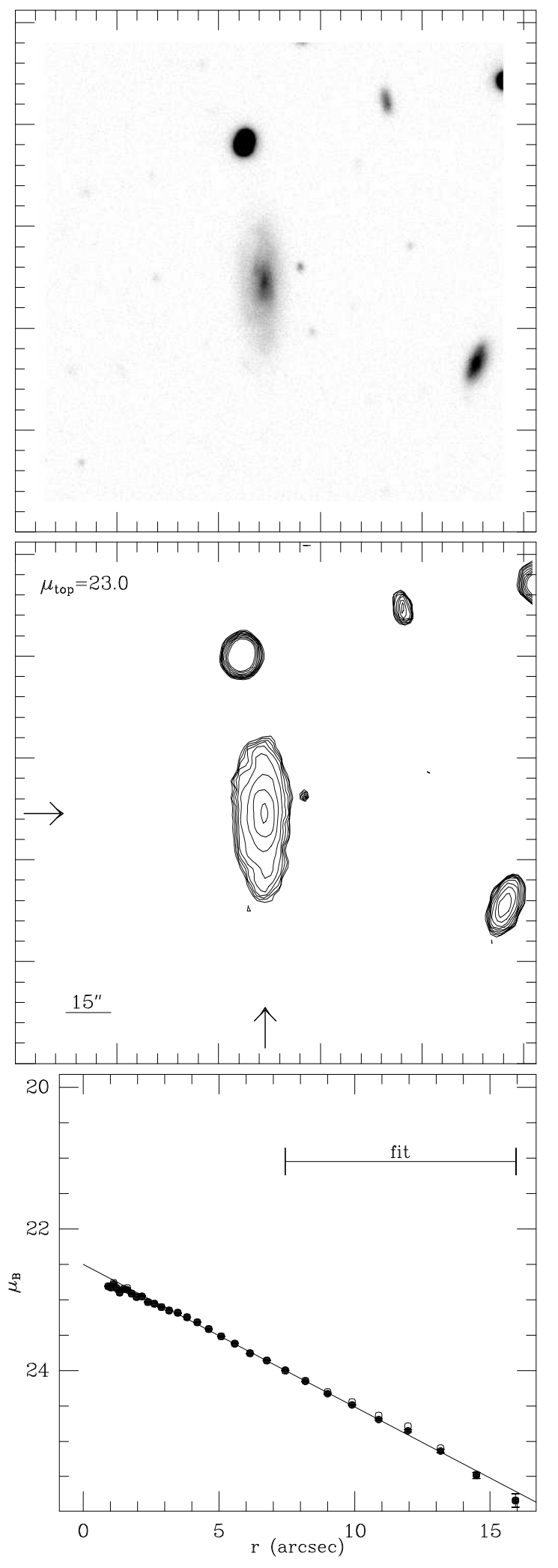

I

75
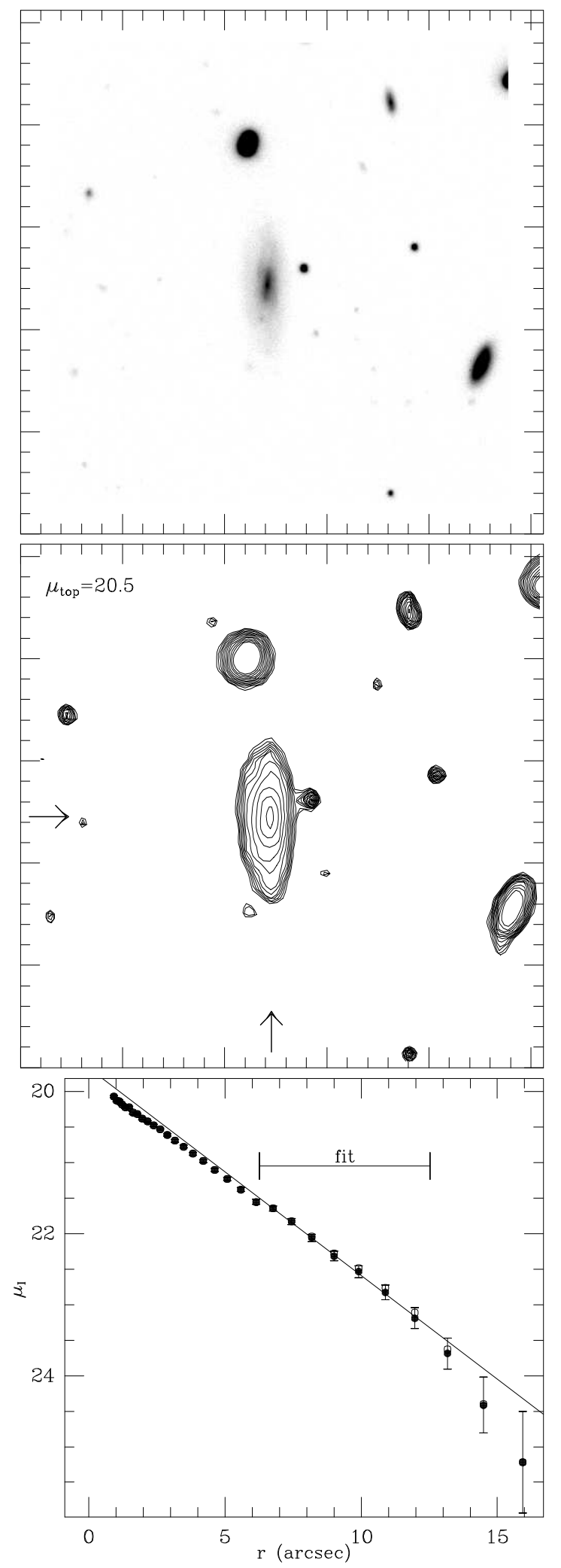

Figure 36: UGC 07295 
B
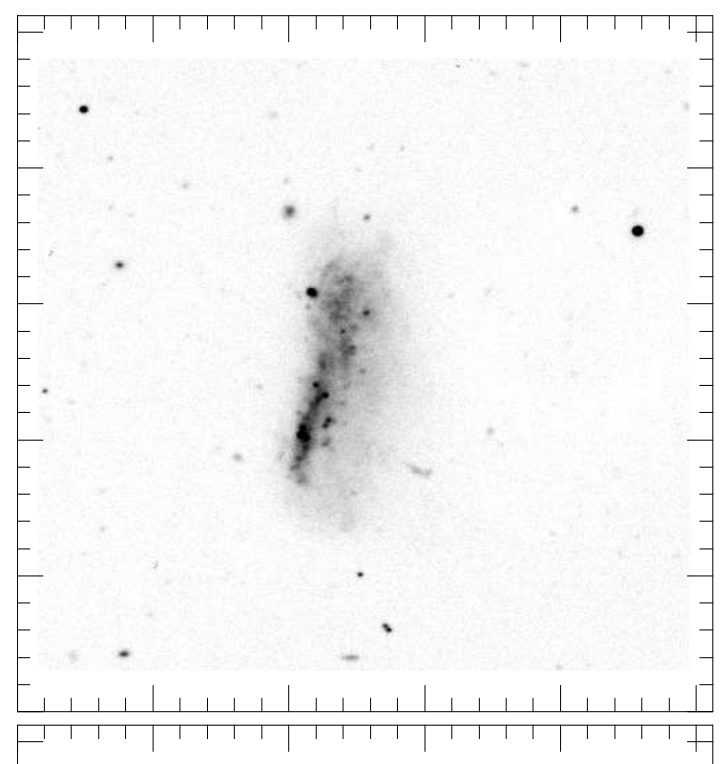
$\mu_{\text {top }}=22.5$
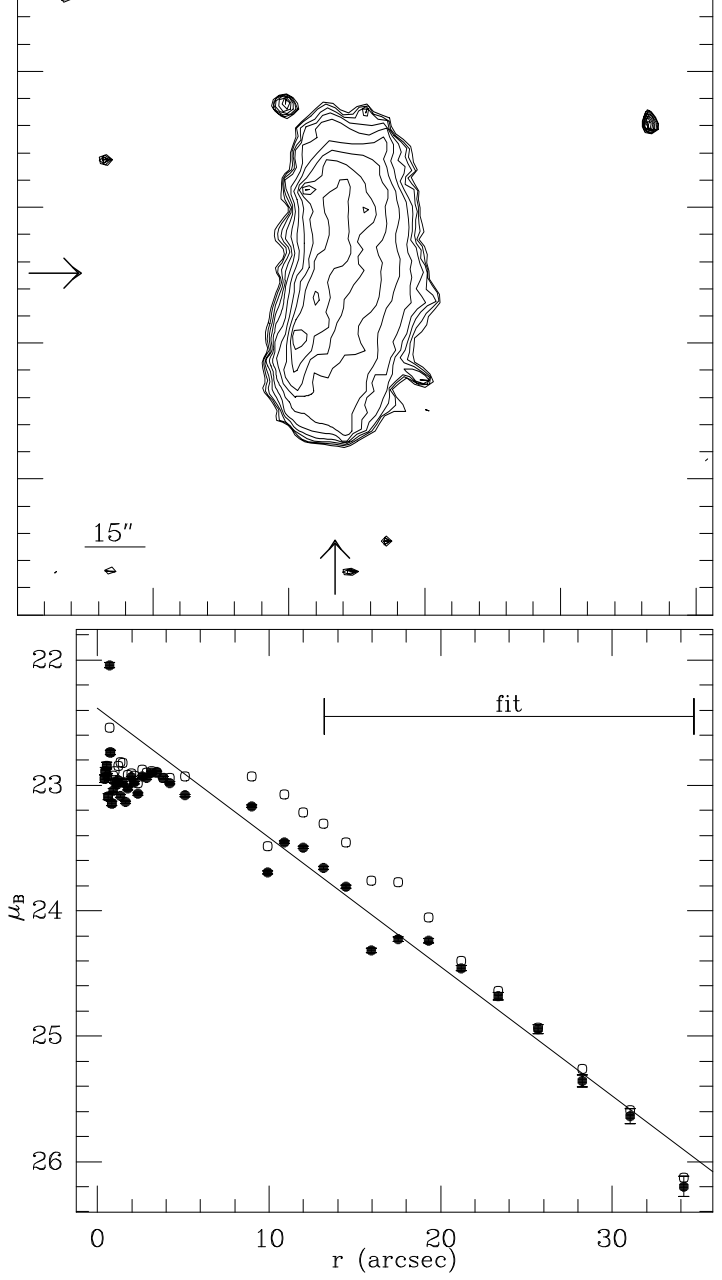

I 76
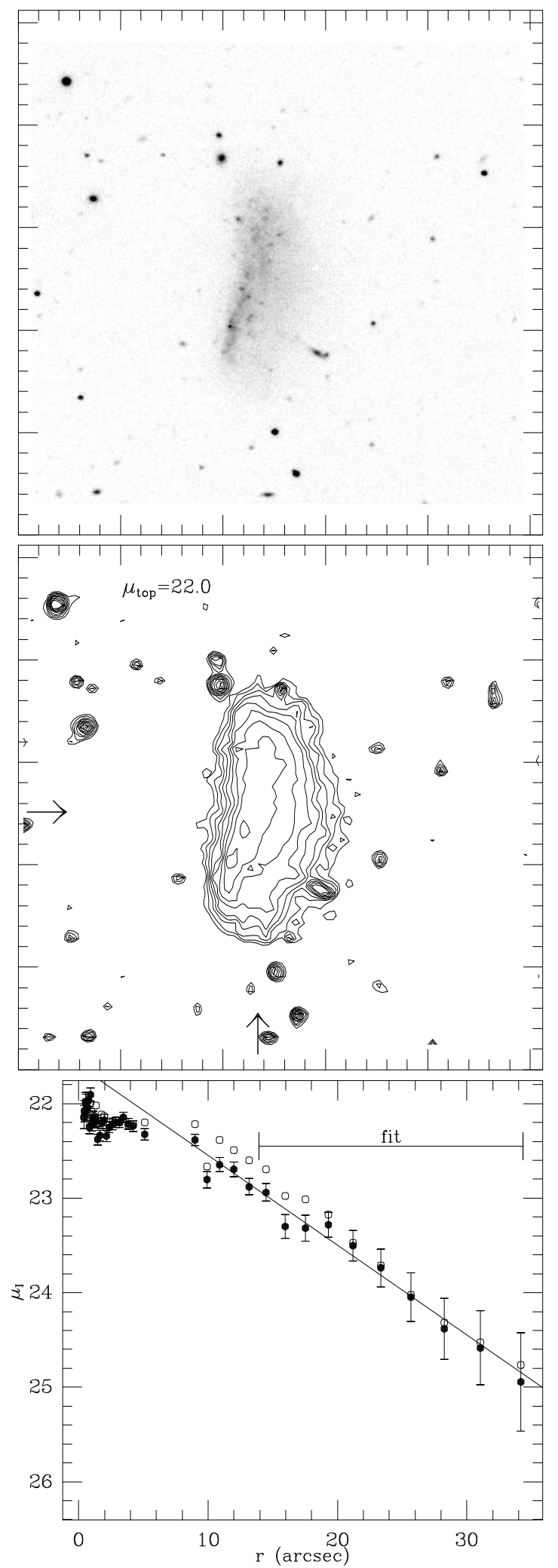

Figure 37: UGC 07548 
B

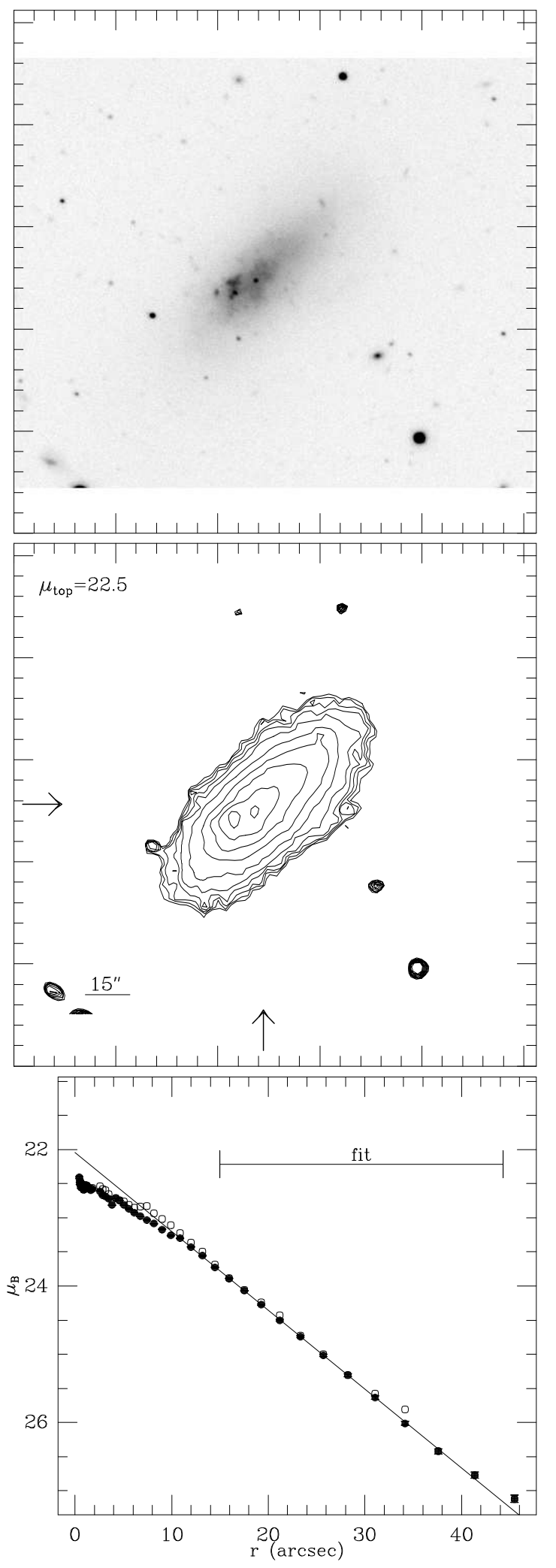

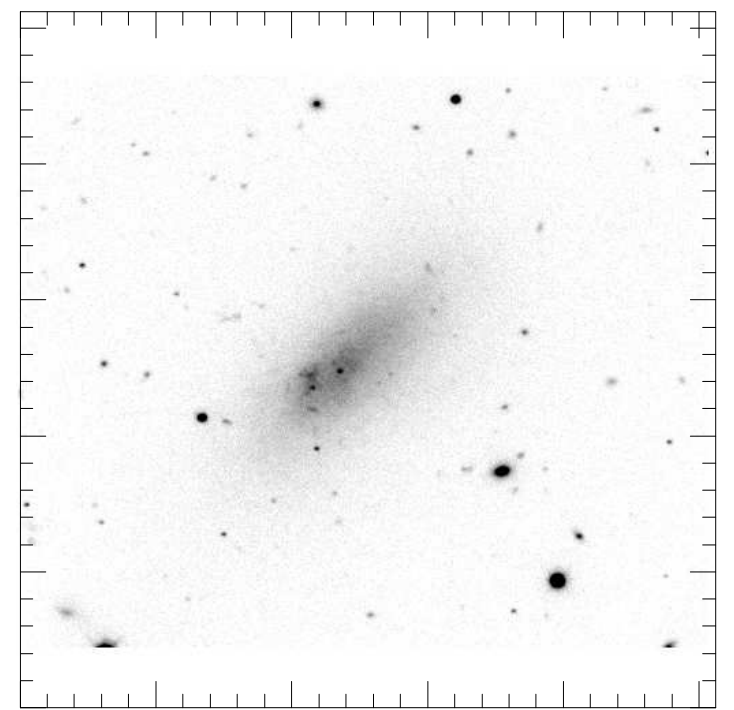
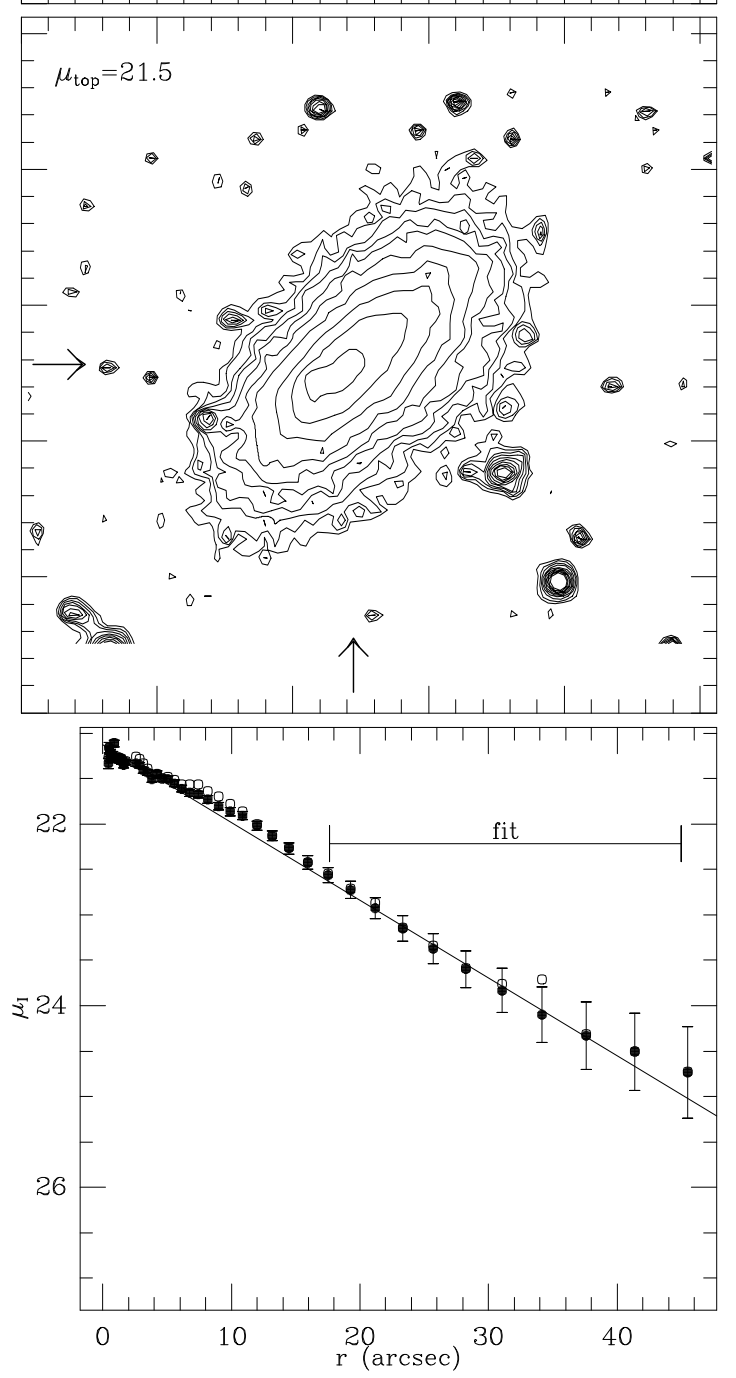

Figure 38: UGC 07596 
B
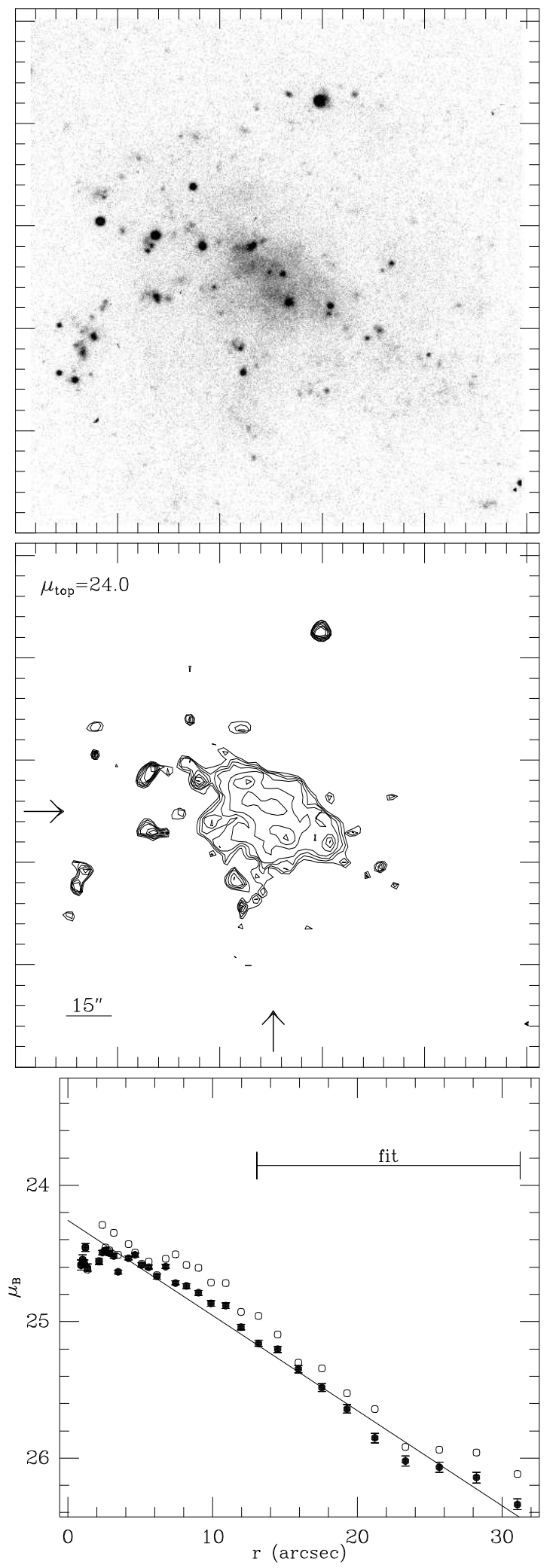

I
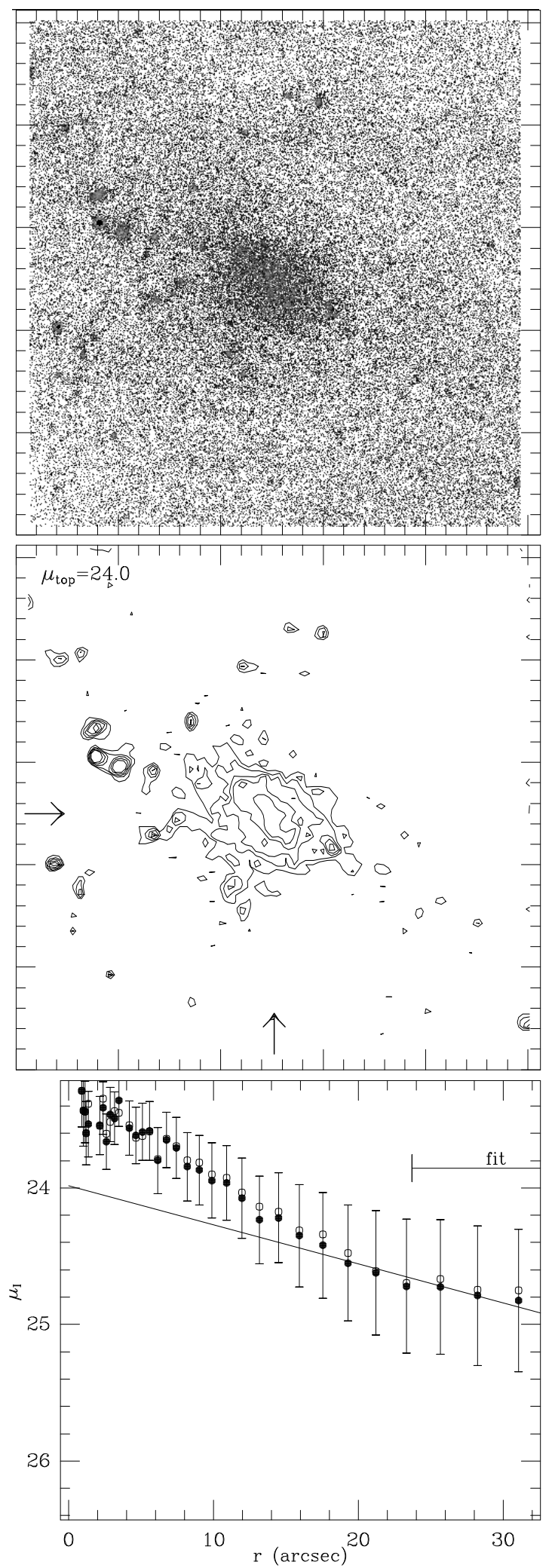

Figure 39: UGC 07608 
B
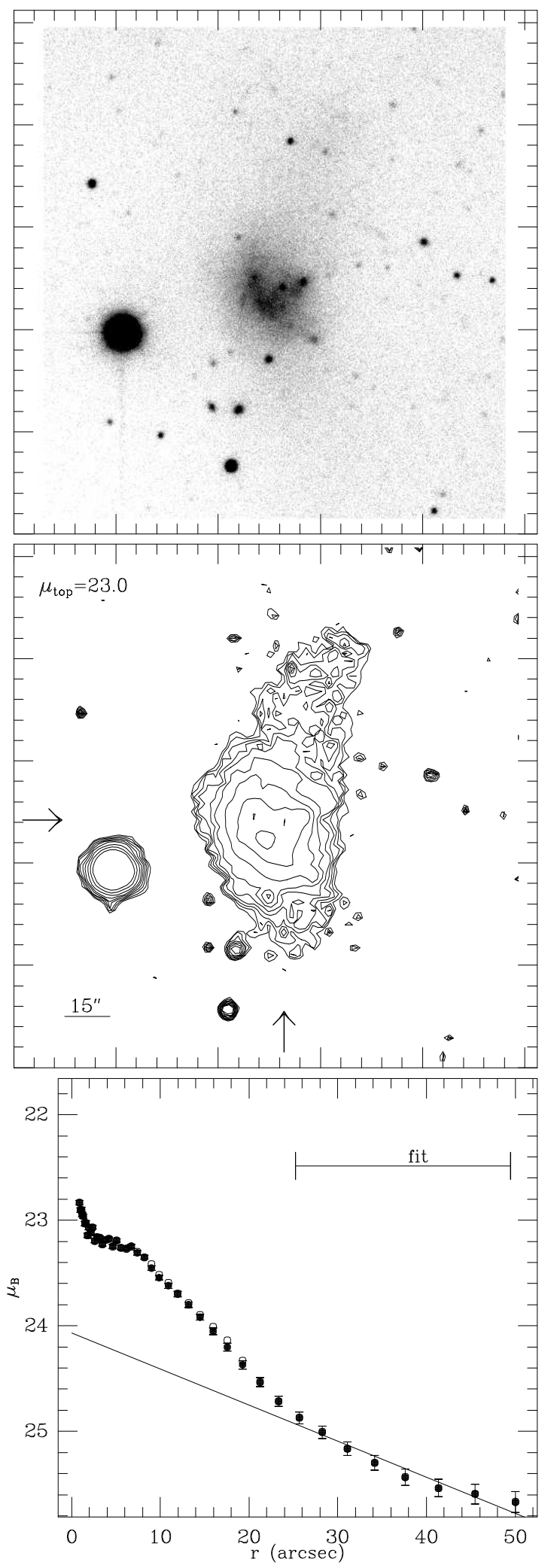

I
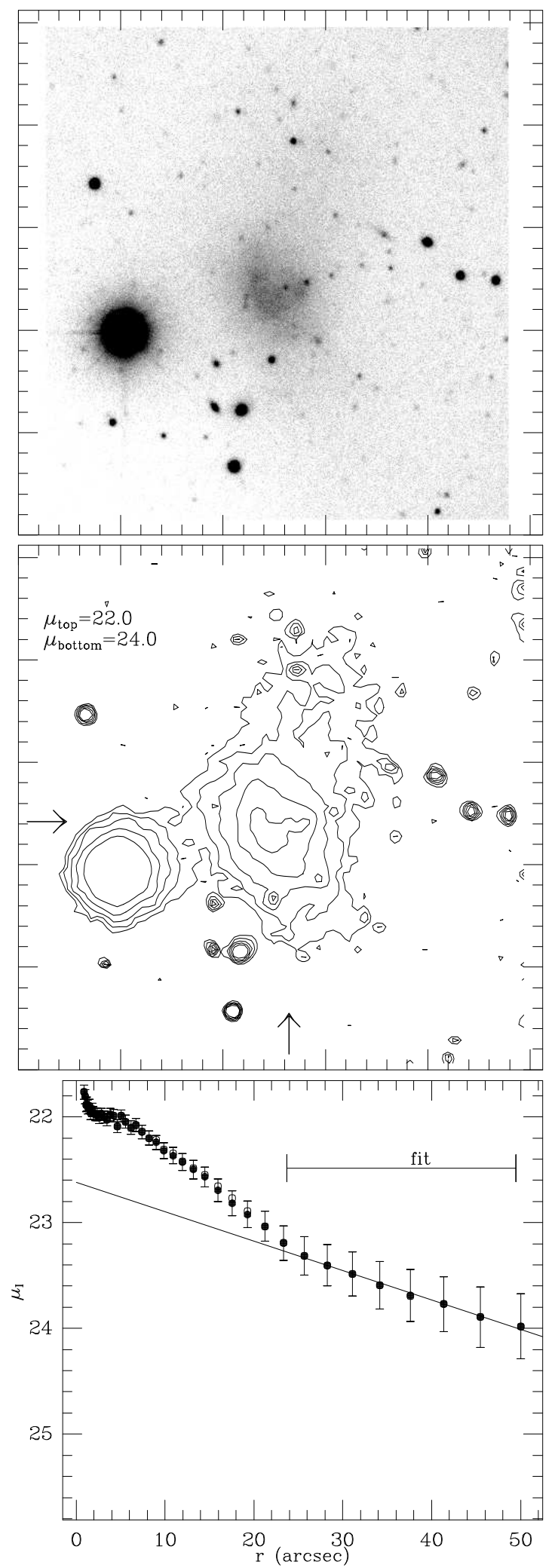

Figure 40: UGC 07636 
B

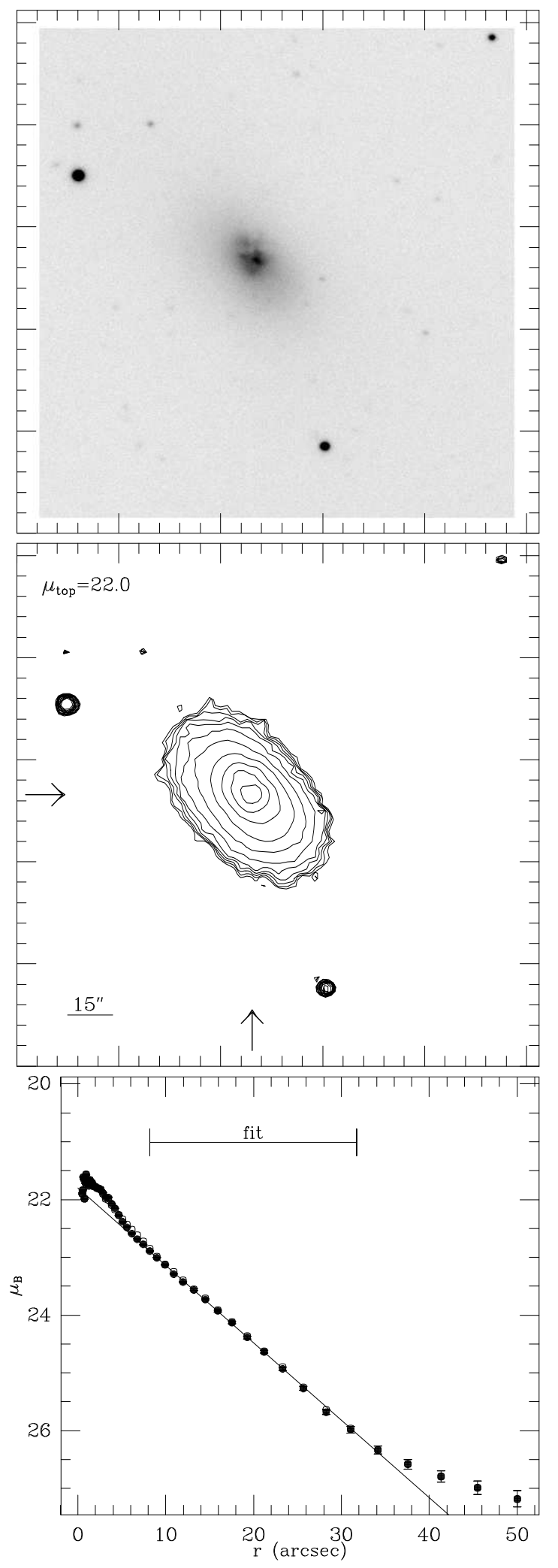

80
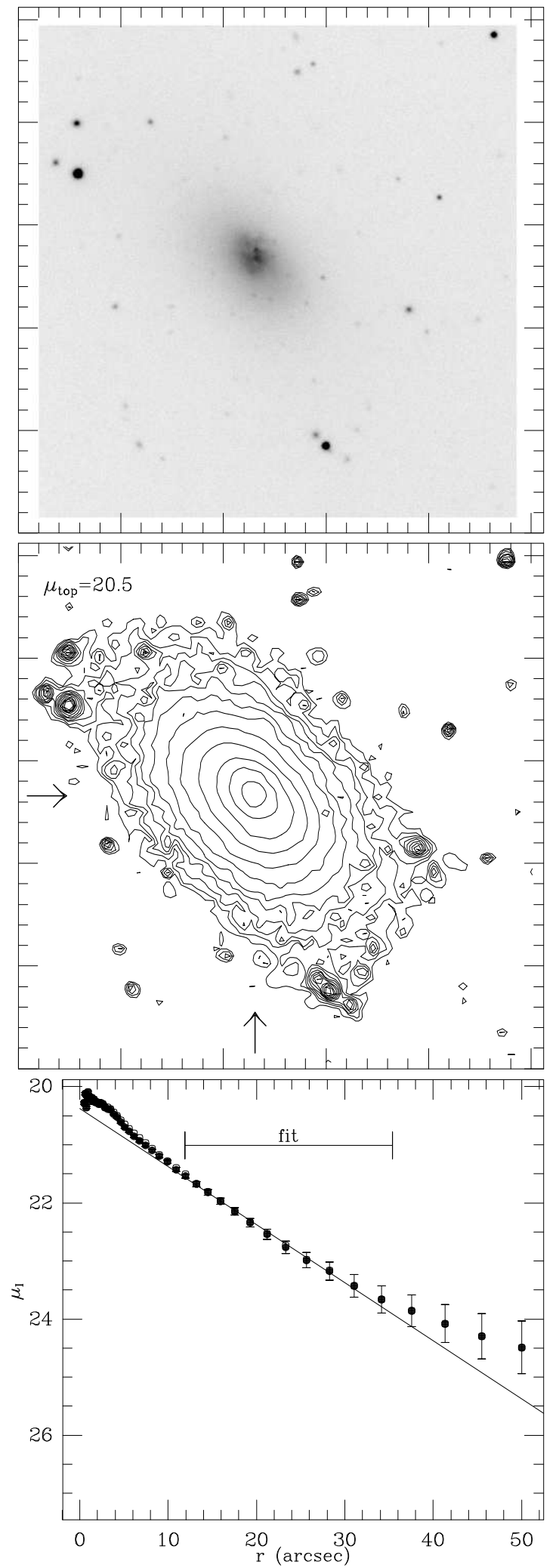

Figure 41: UGC 07684 
B

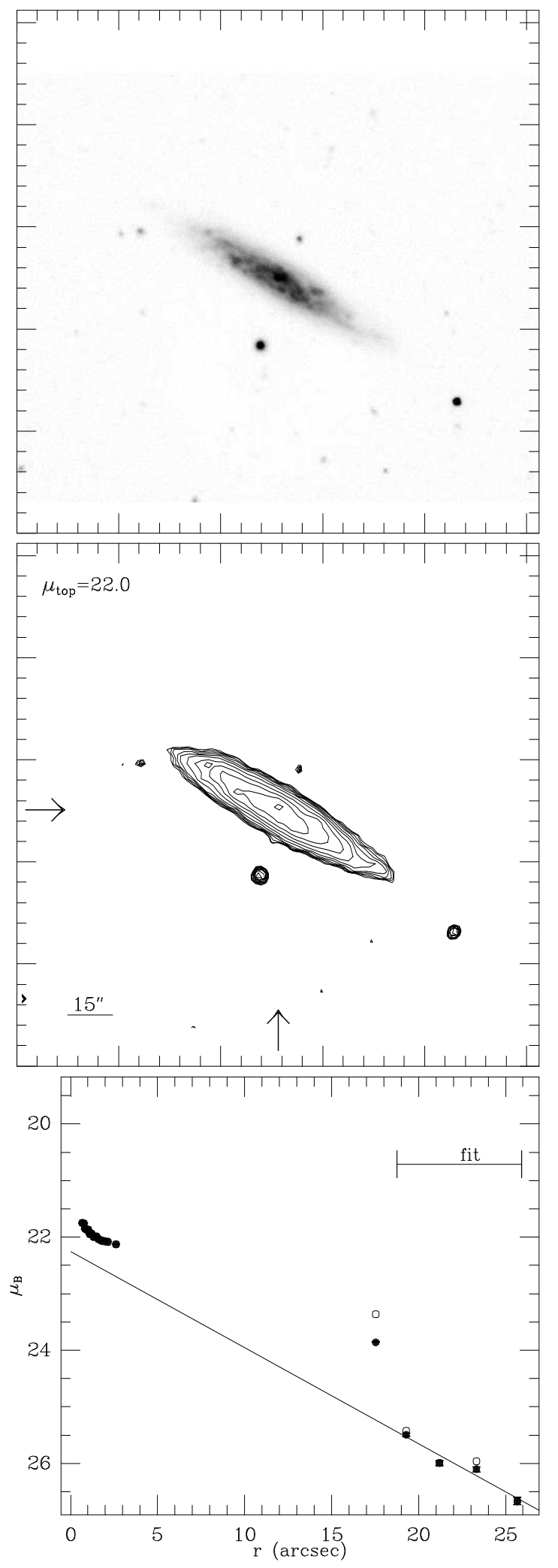

I

81
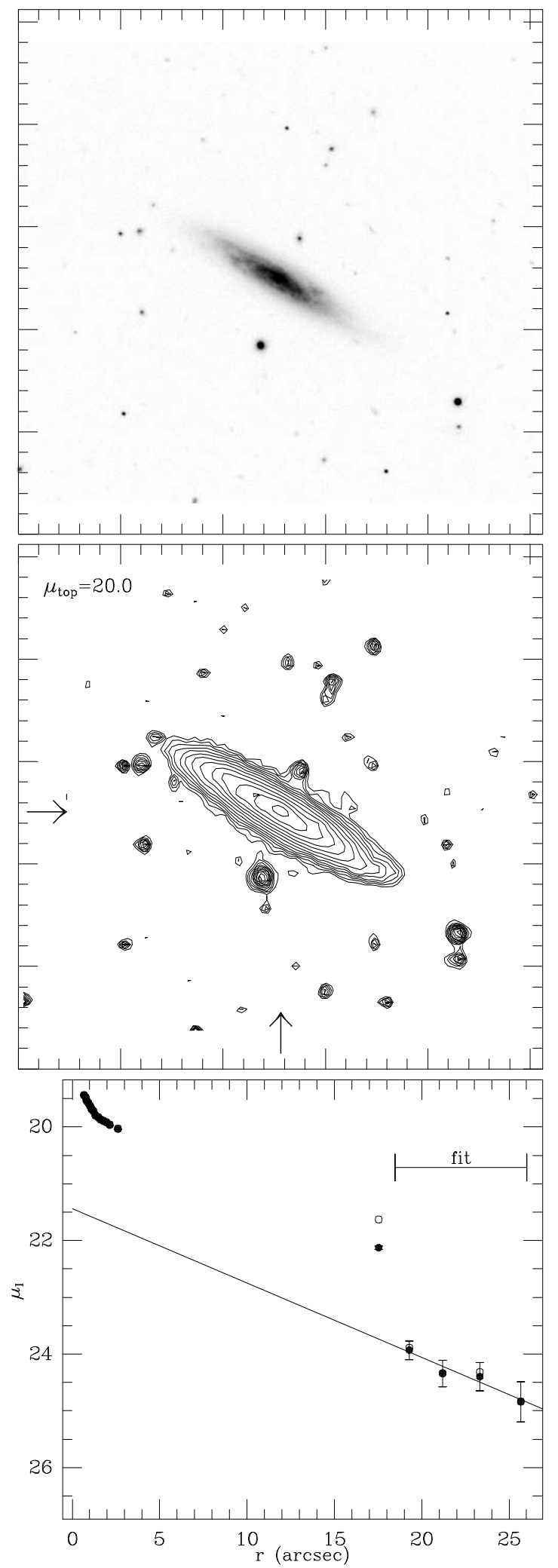

Figure 42: UGC 07882 
B

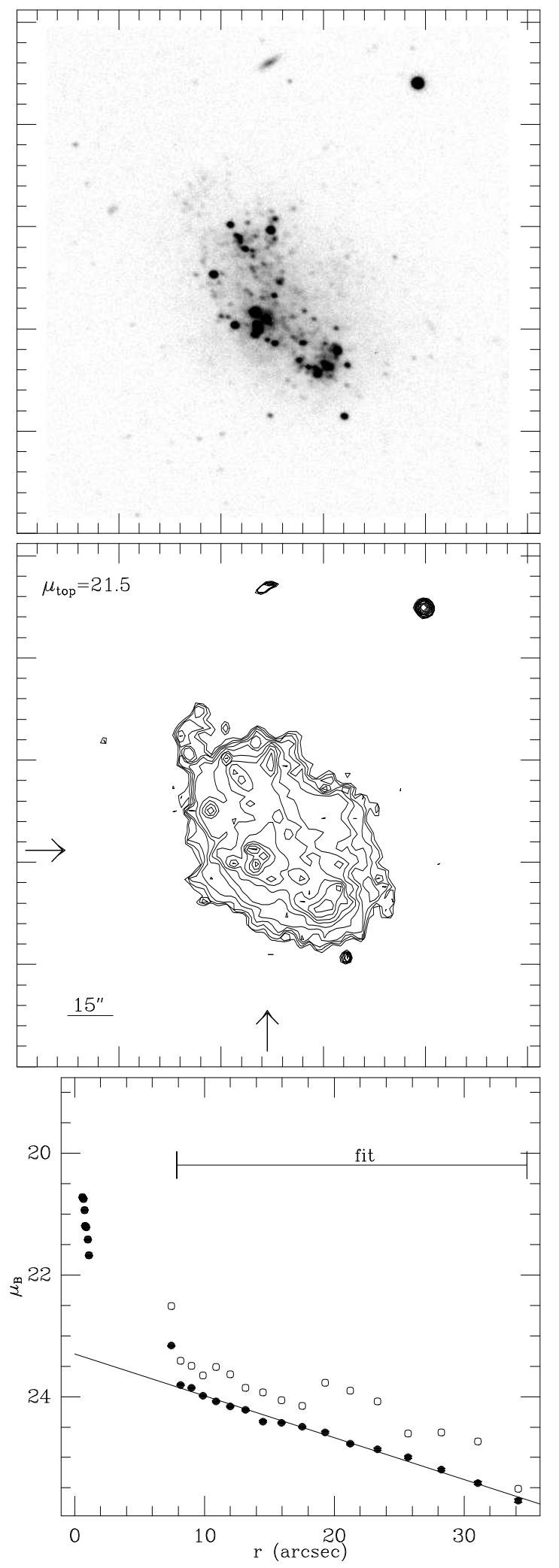

I

82
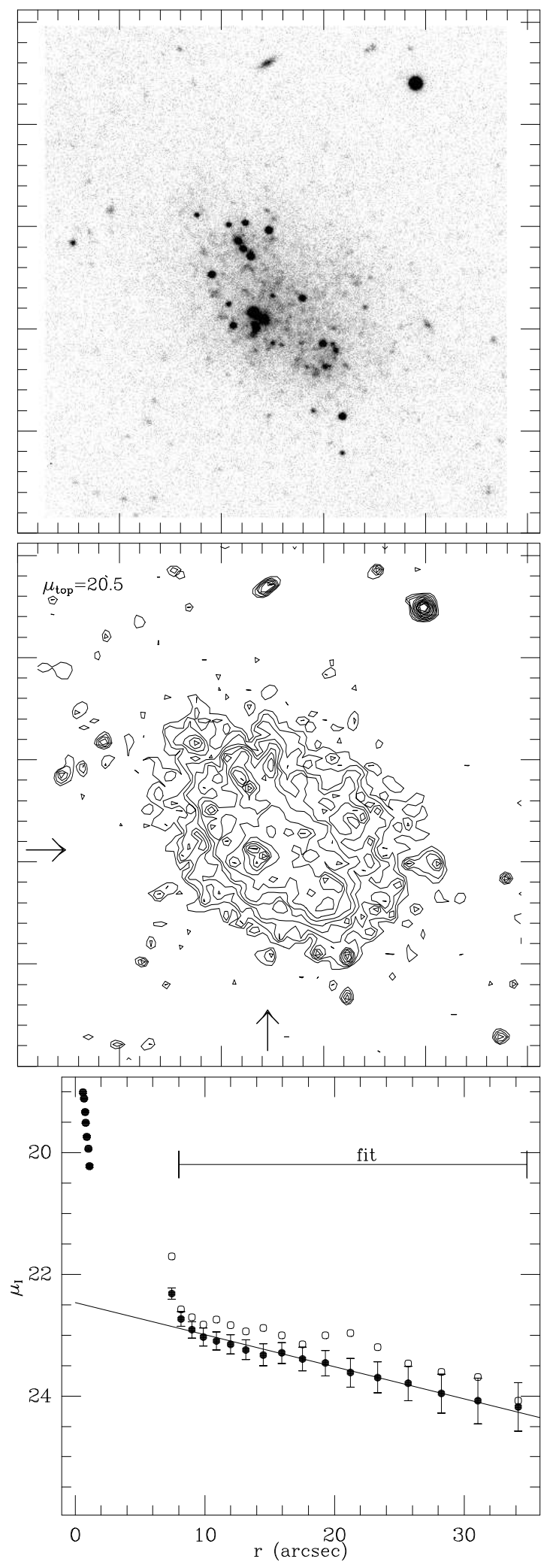

Figure 43: UGC 08091 (GR8) 
B

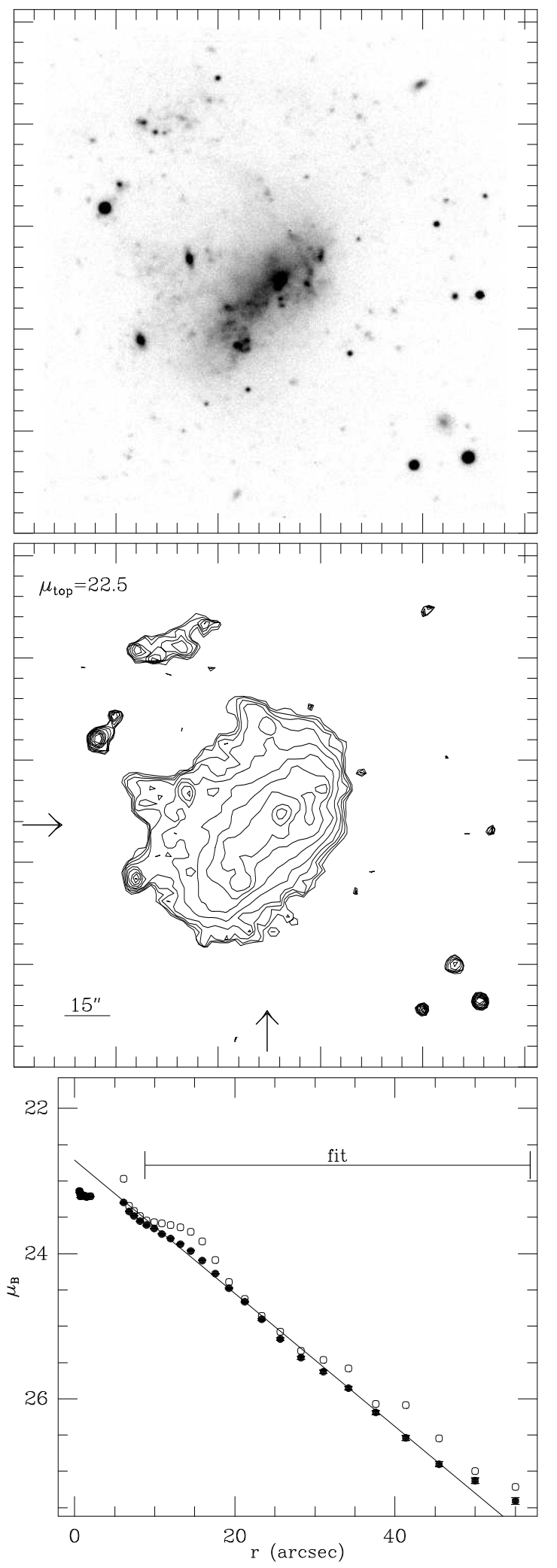

I

83
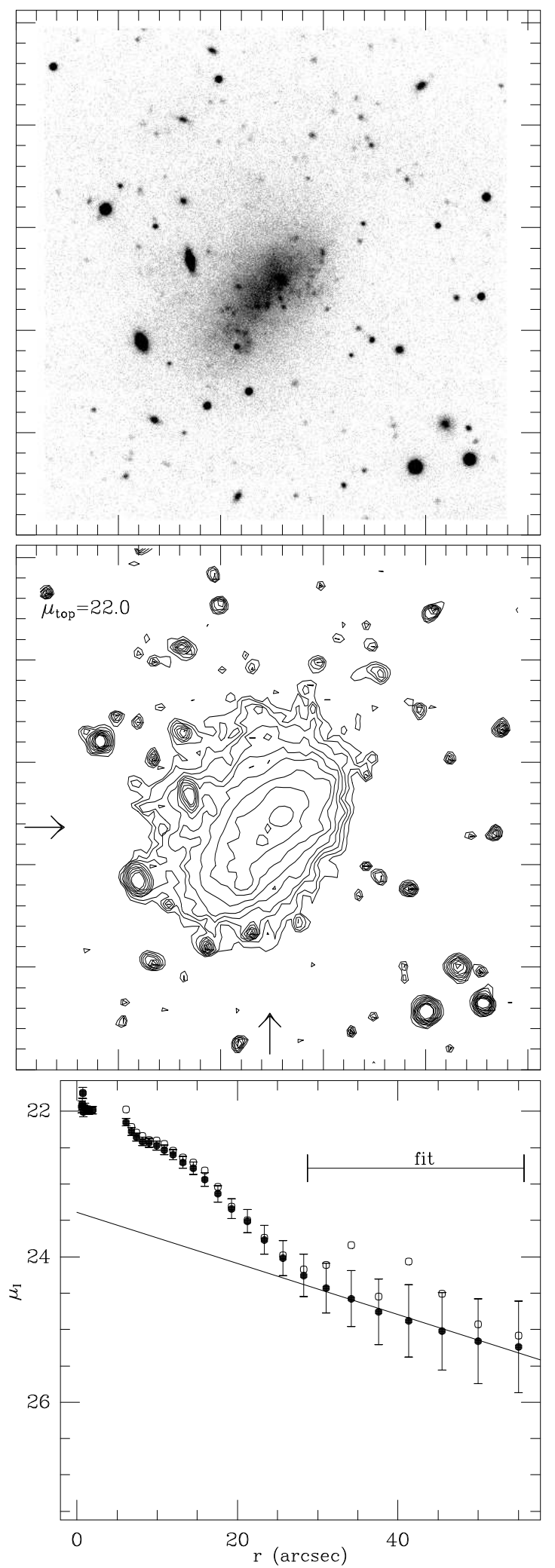

Figure 44: UGC 08683 
B
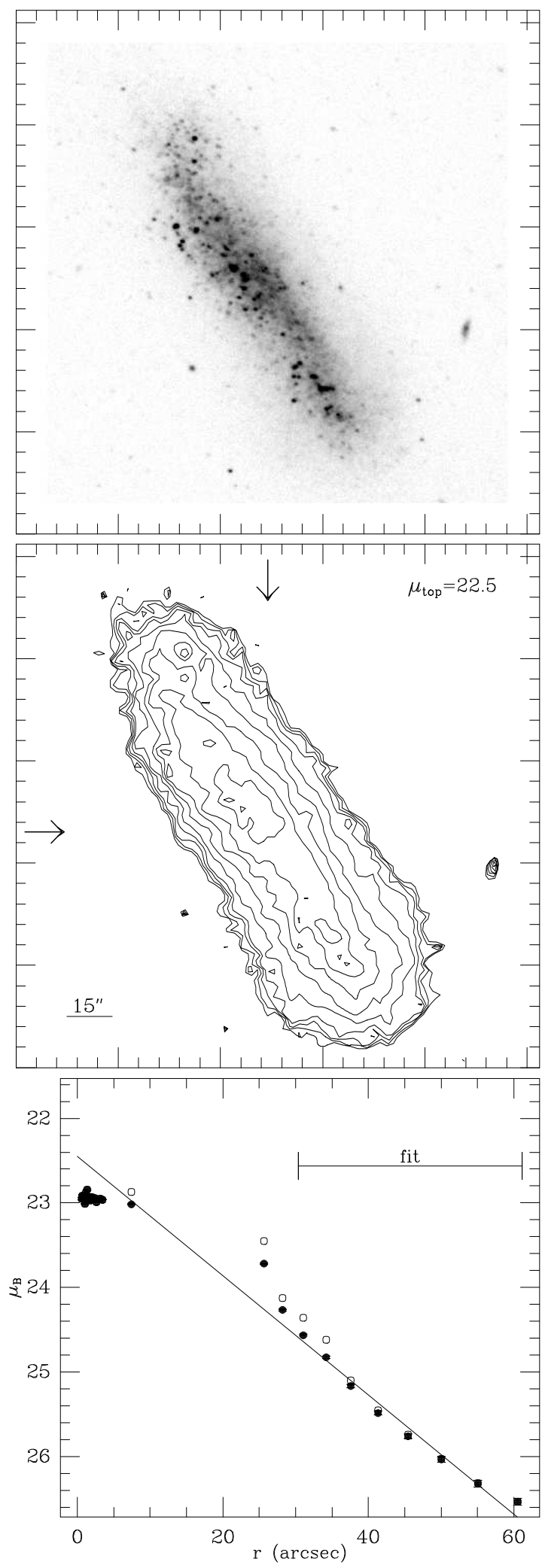

I 84
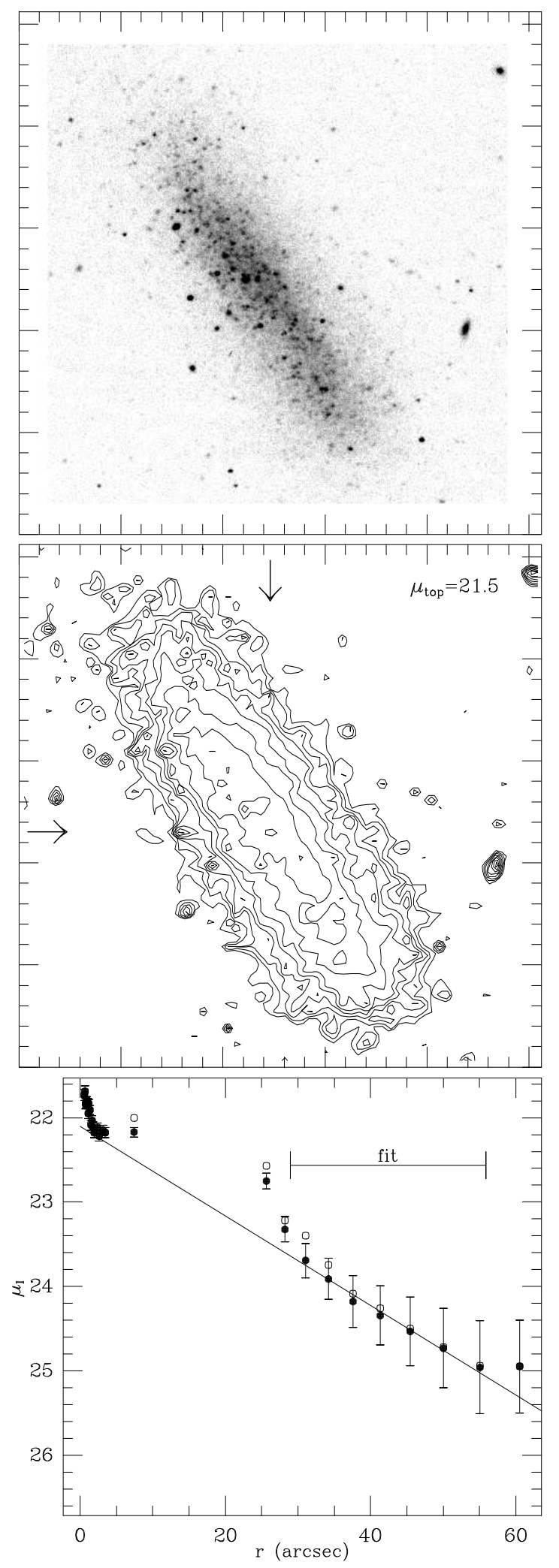

Figure 45: UGC 08760 
B
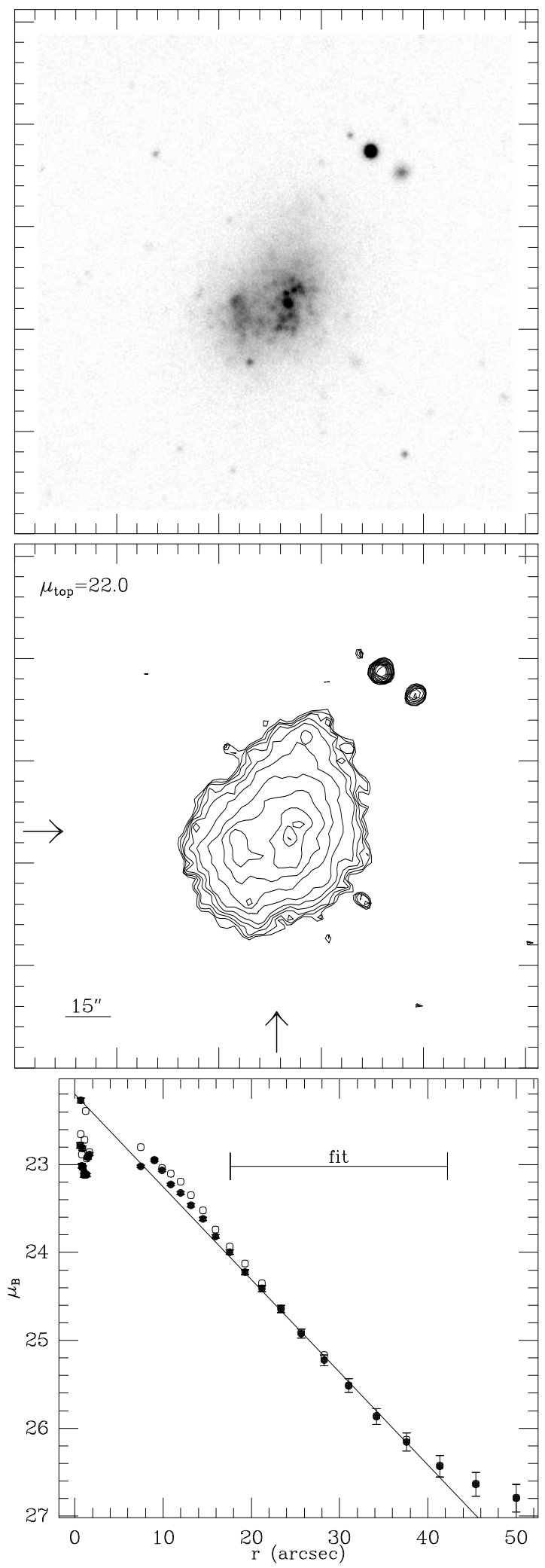

I

85
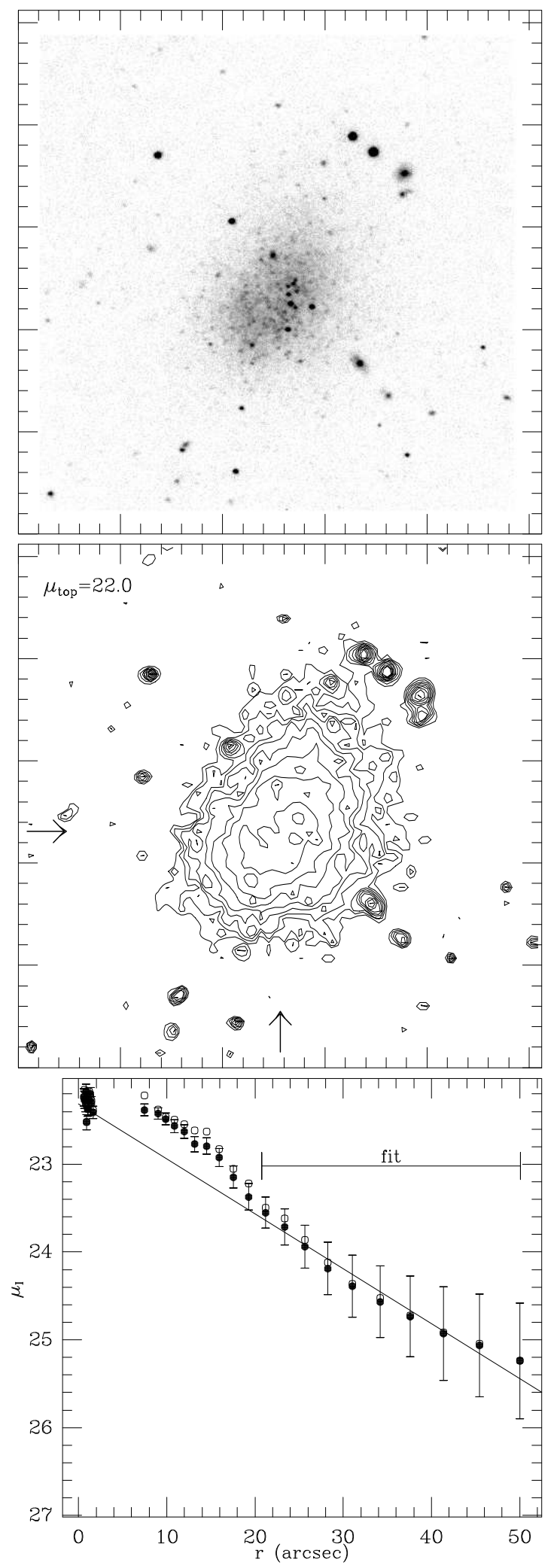

Figure 46: UGC 08833 
B

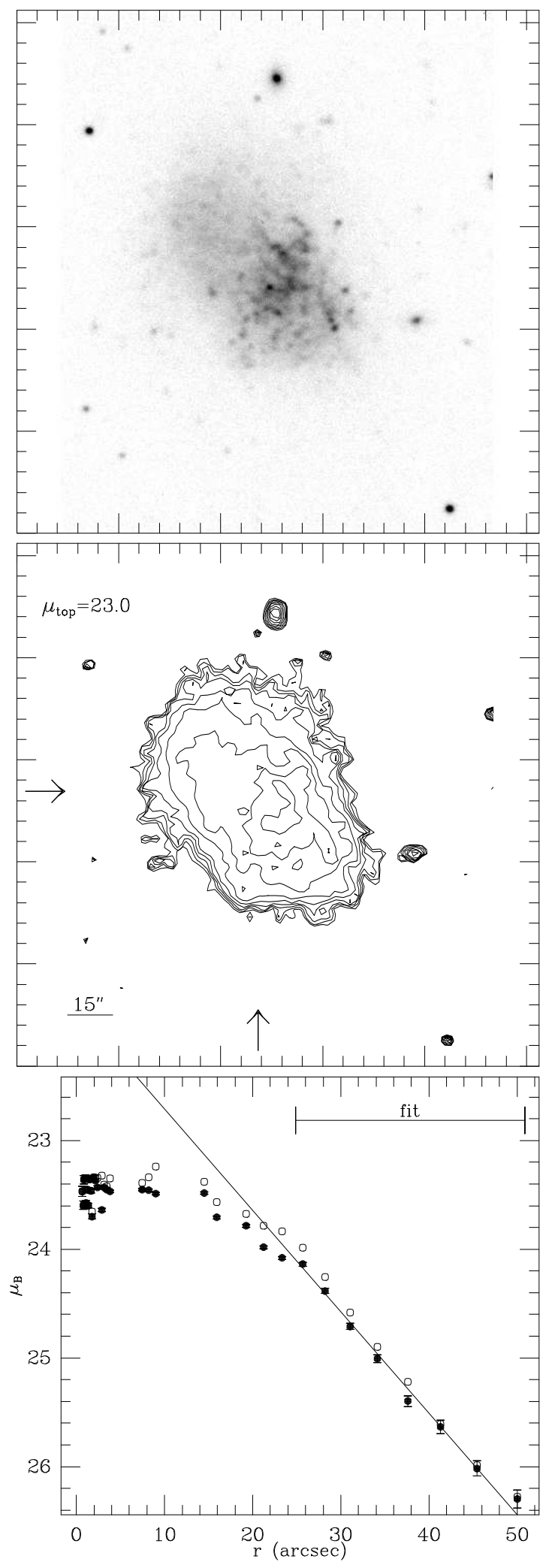

I

86
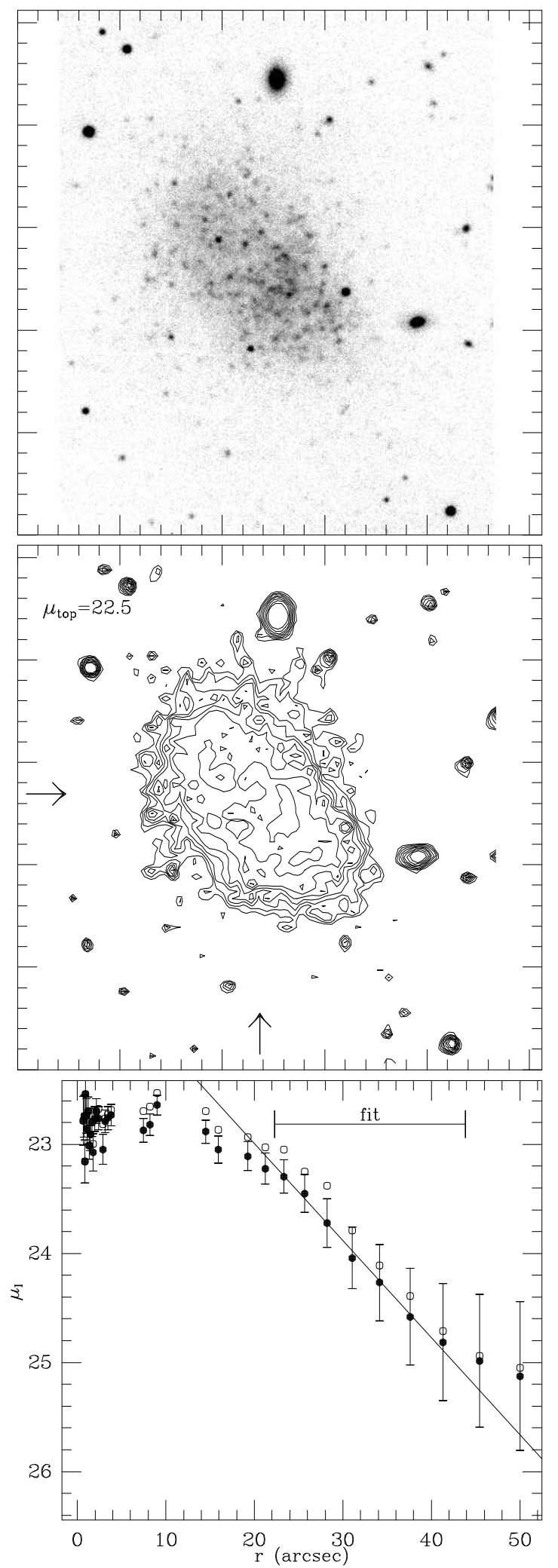

Figure 47: UGC 09128 
B

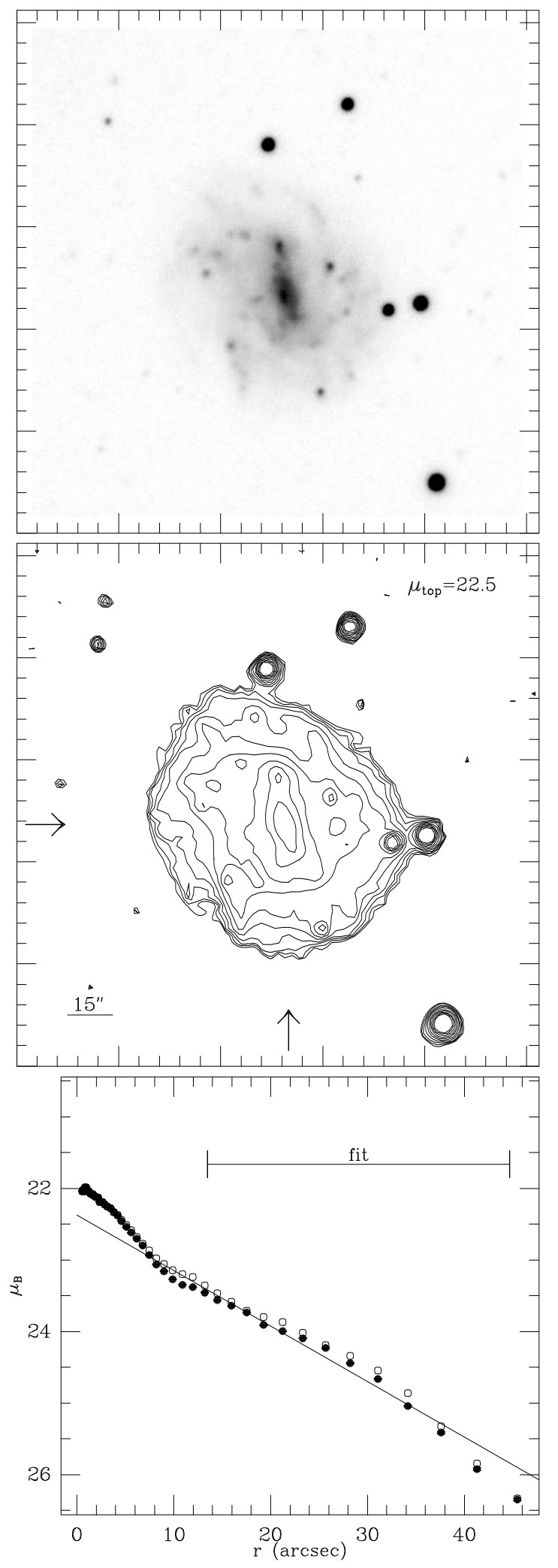

I

87
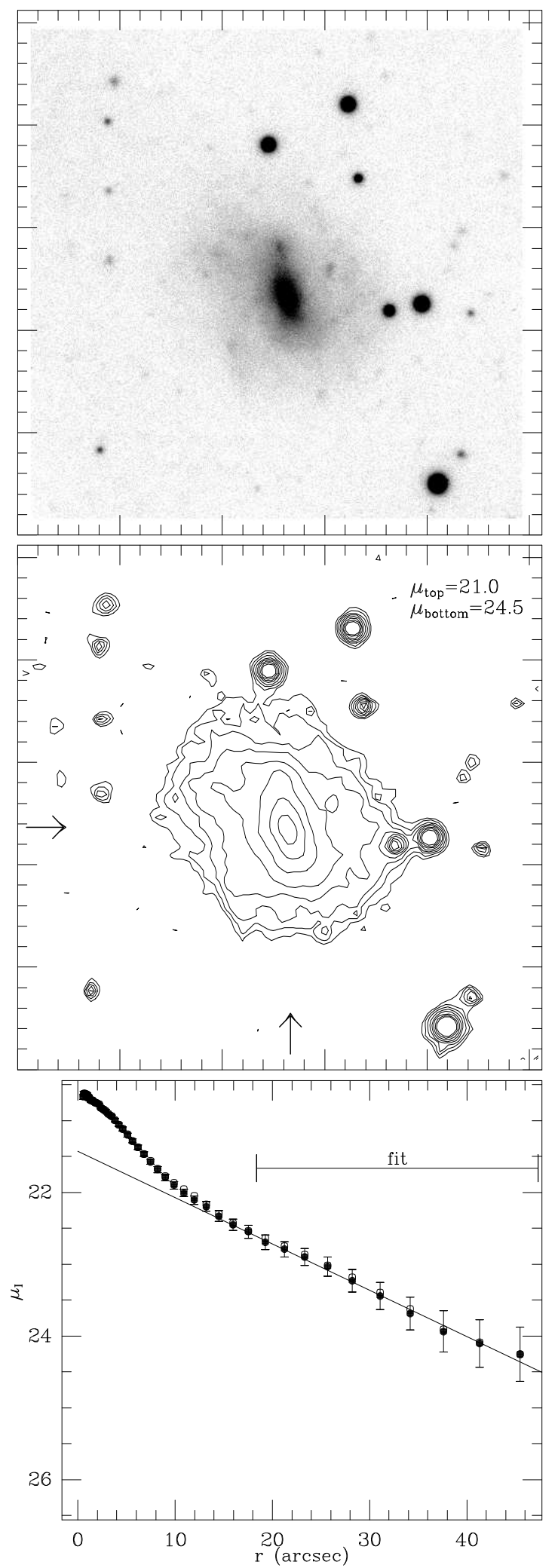

Figure 48: UGC 09391 
B

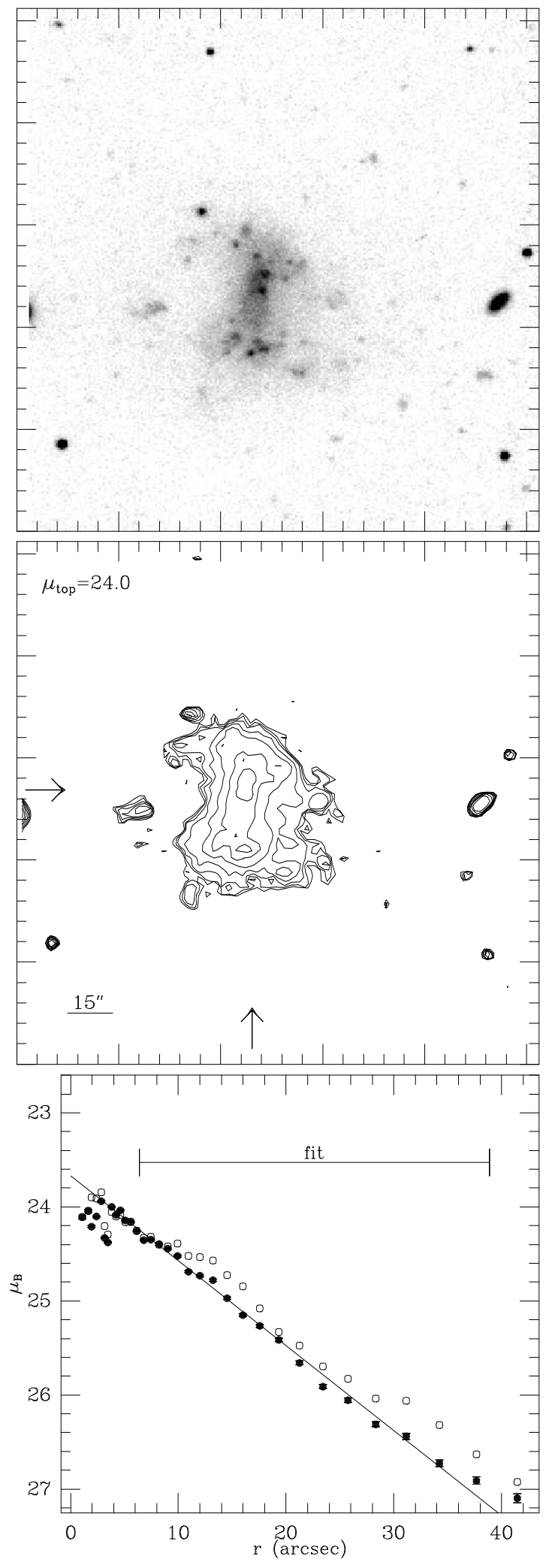

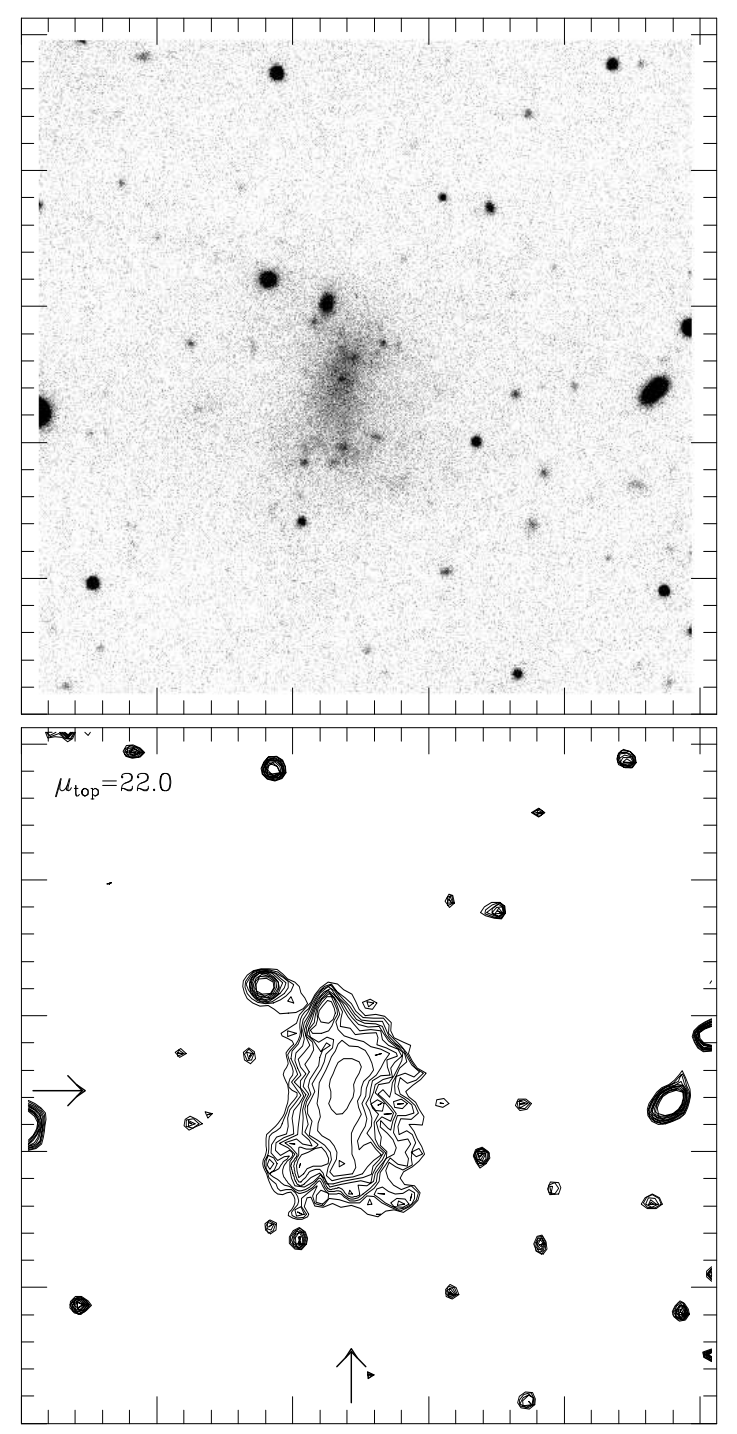

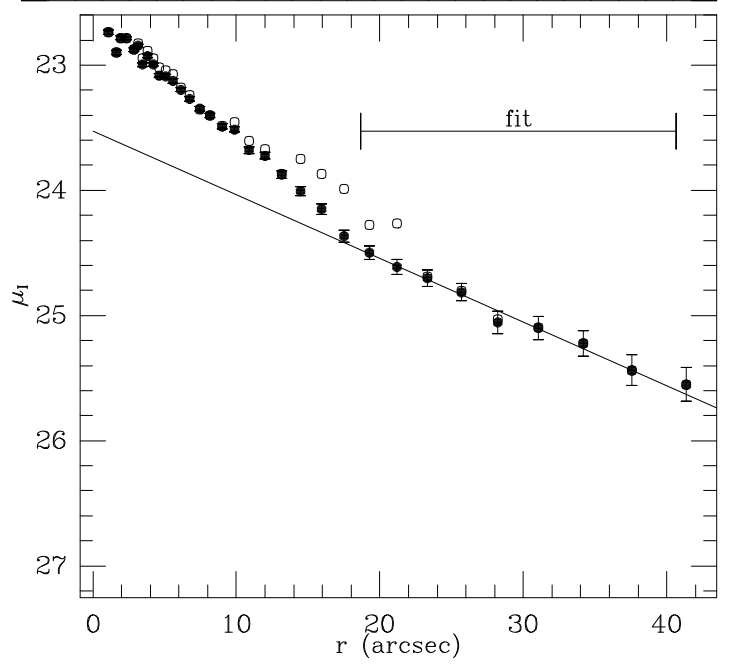

Figure 49: UGC 10031 (B-May85) 
B

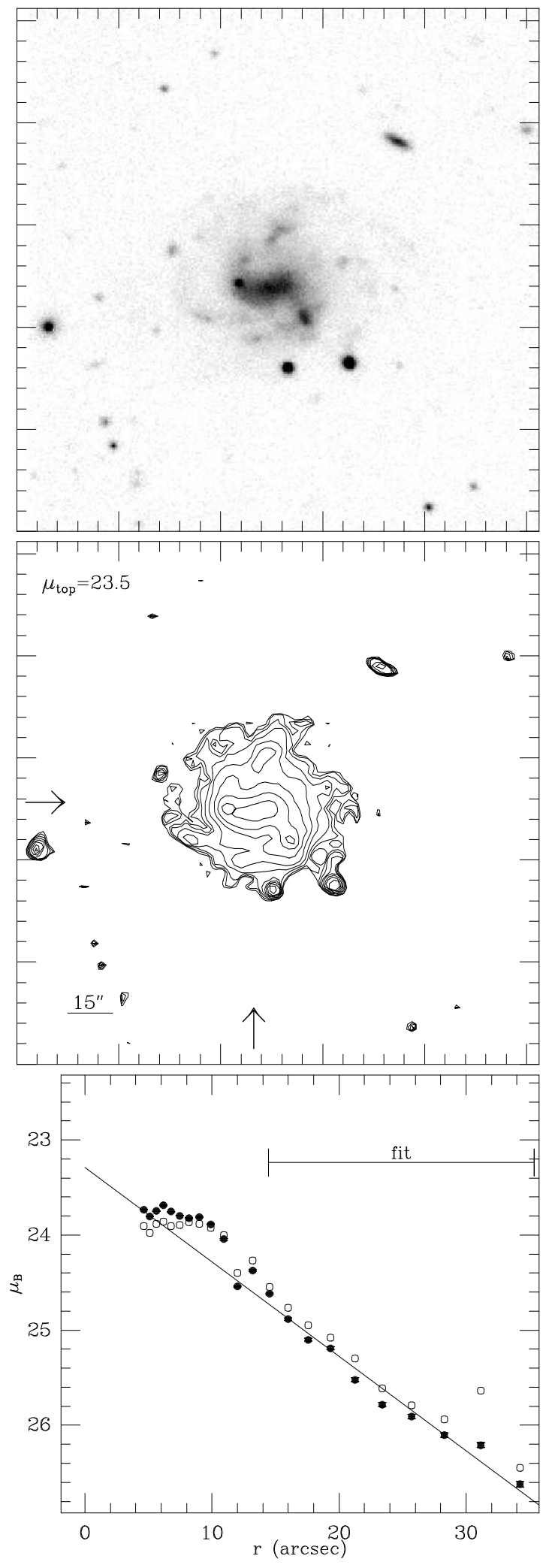

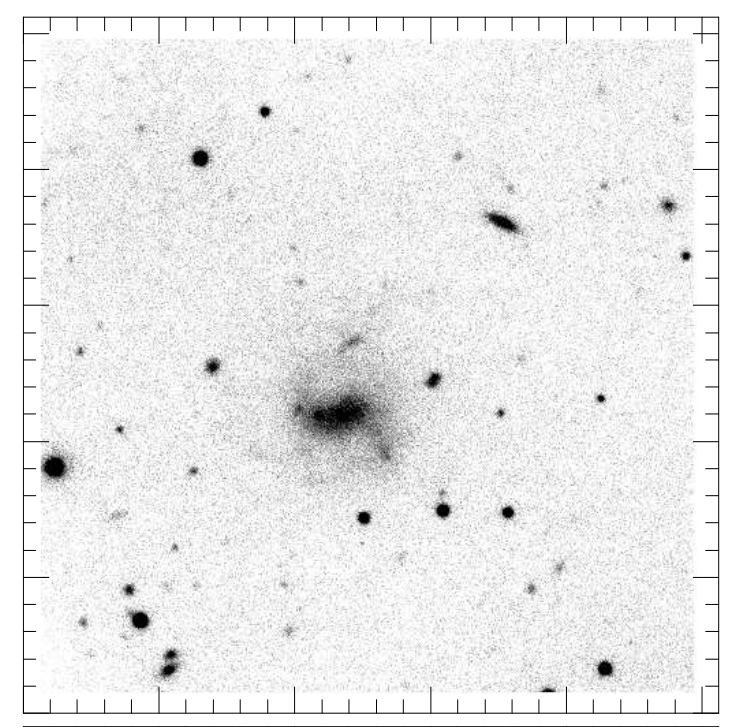
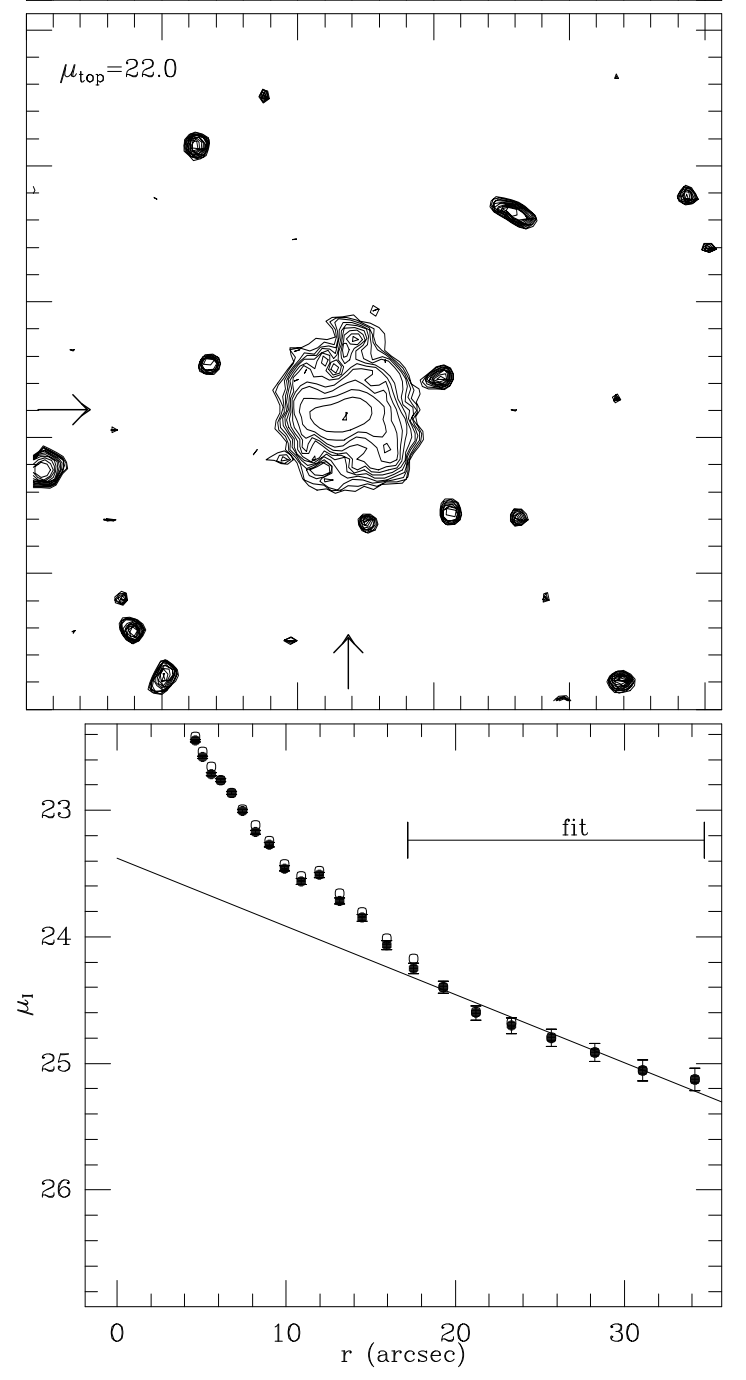

Figure 50: UGC 10058 (B-May85) 
B

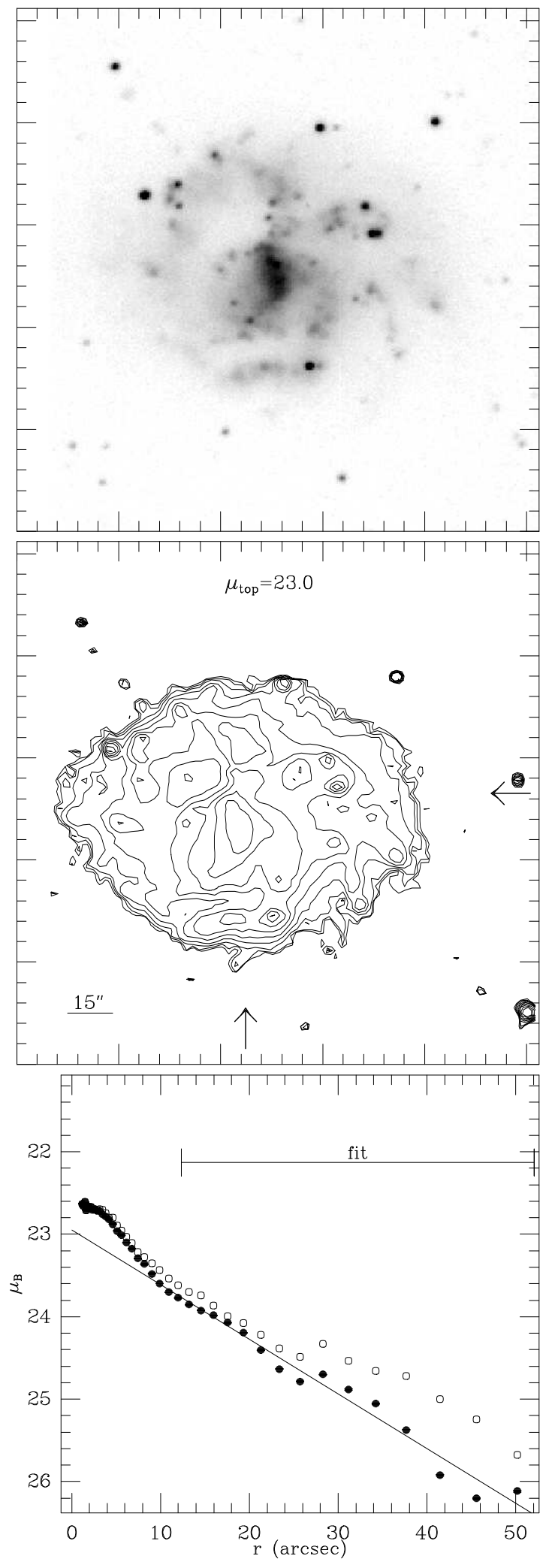

I

90
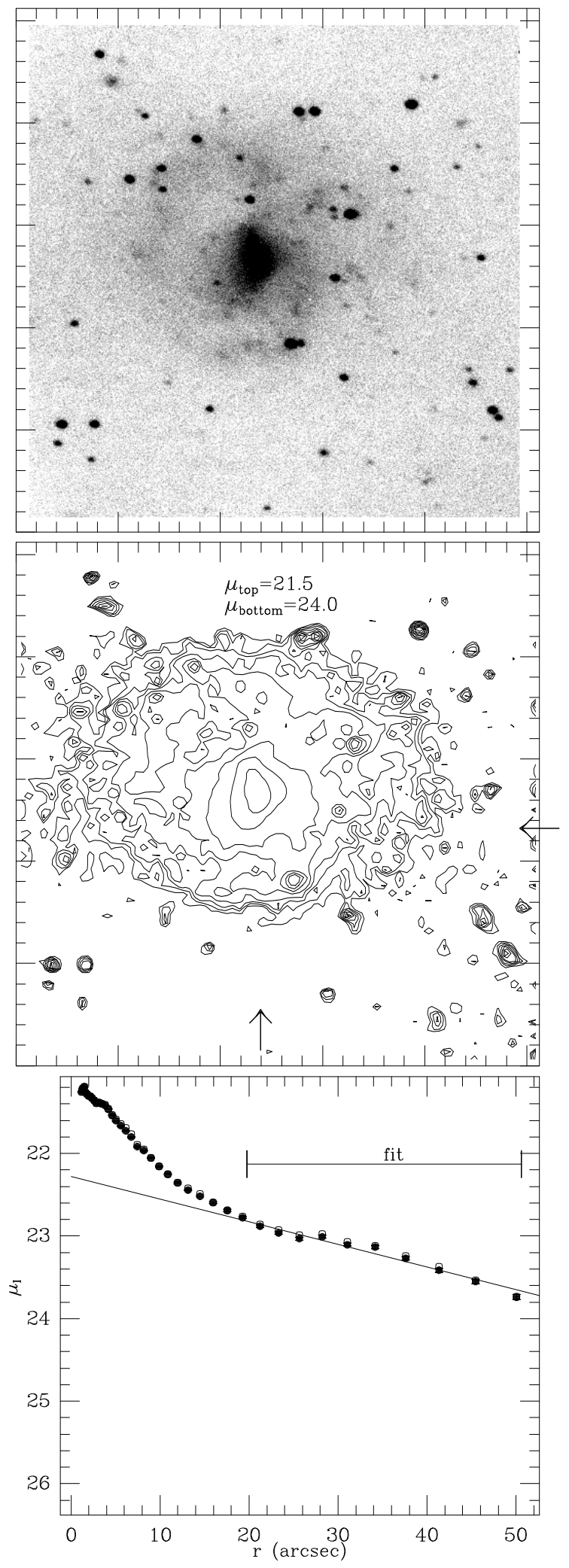

Figure 51: UGC 10290 (B-May85) 
B

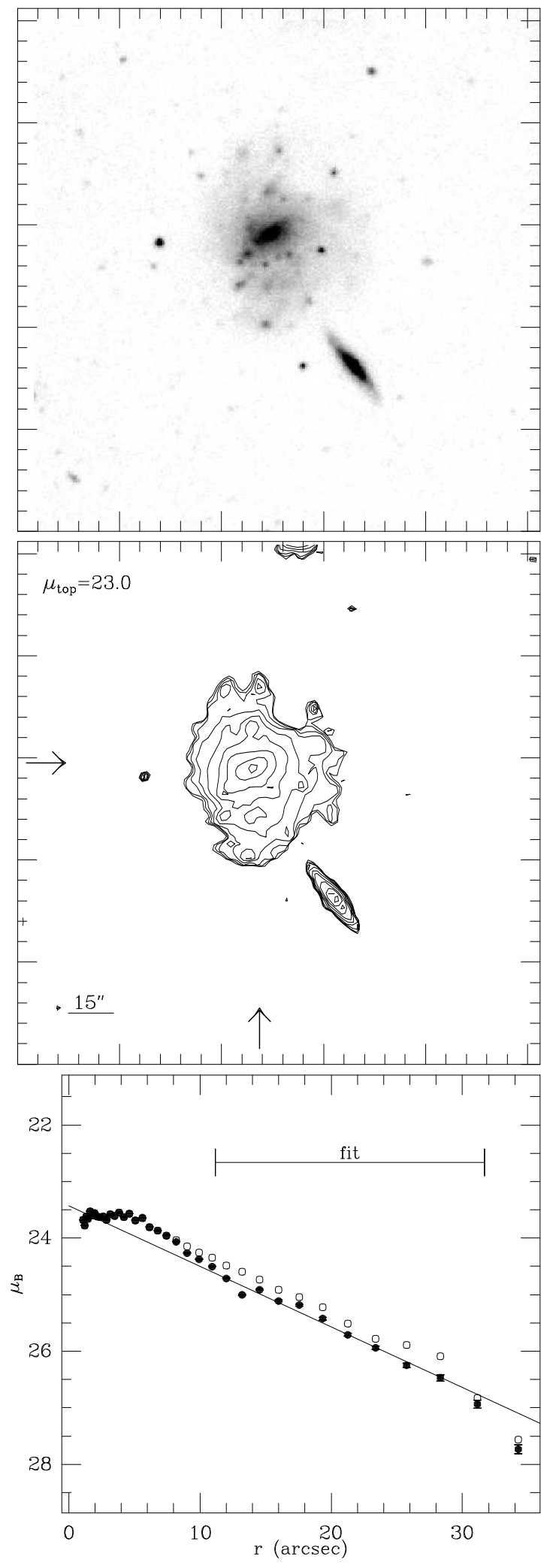

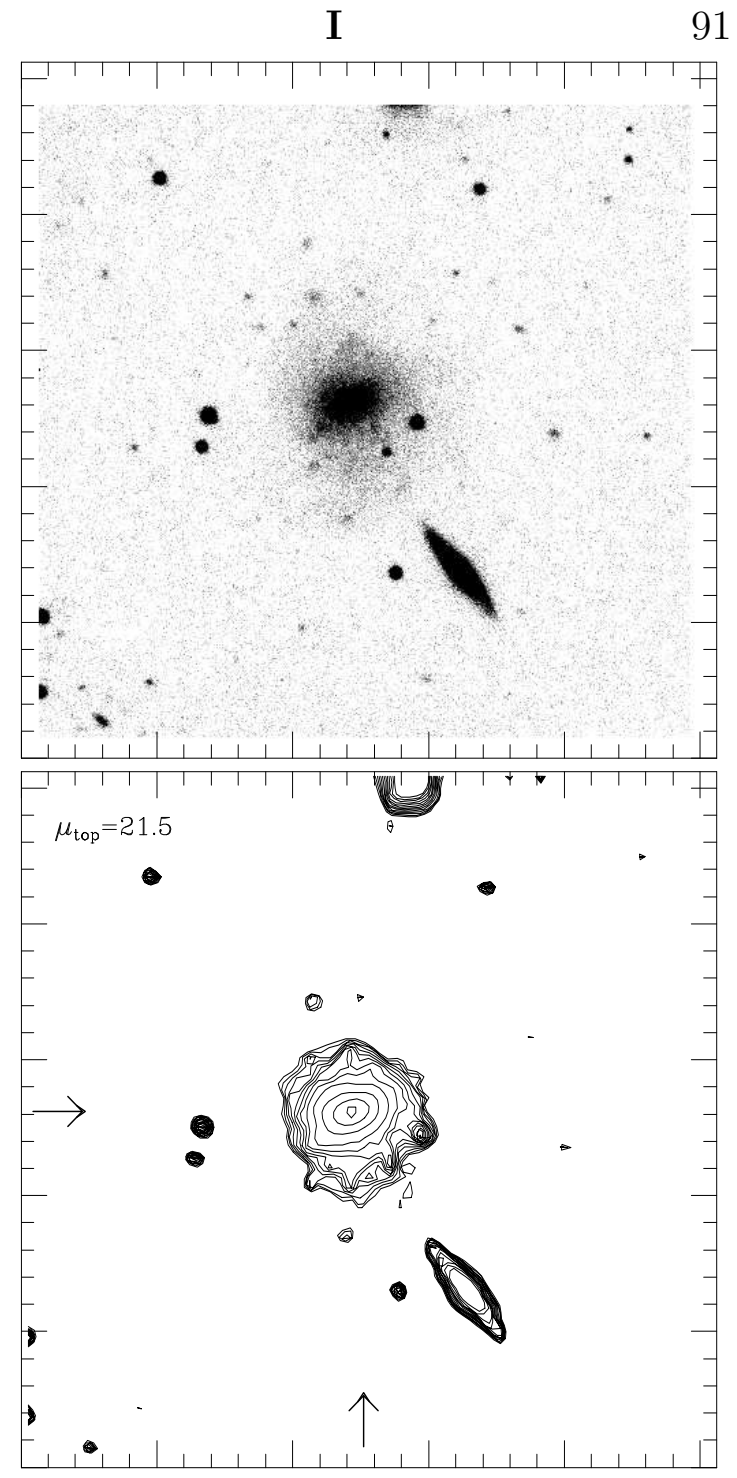

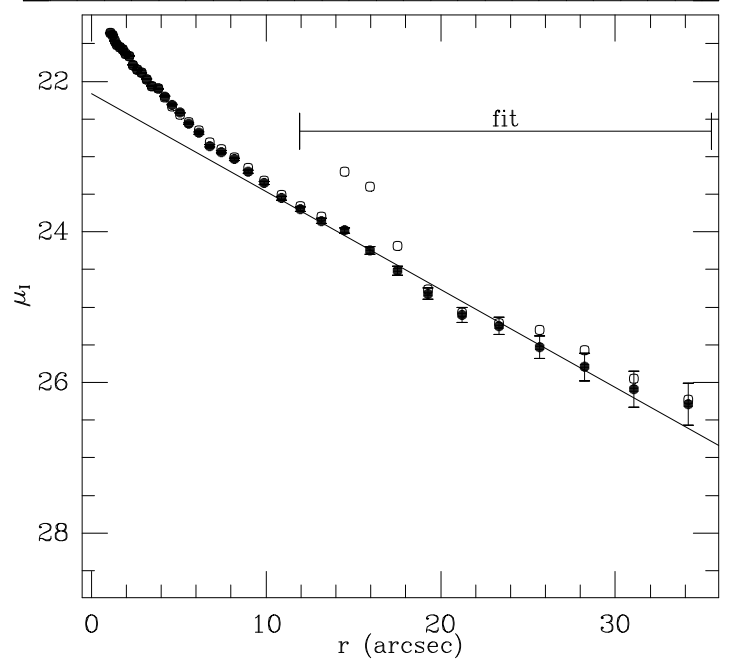

Figure 52: UGC 10376 (B-May85) 
B
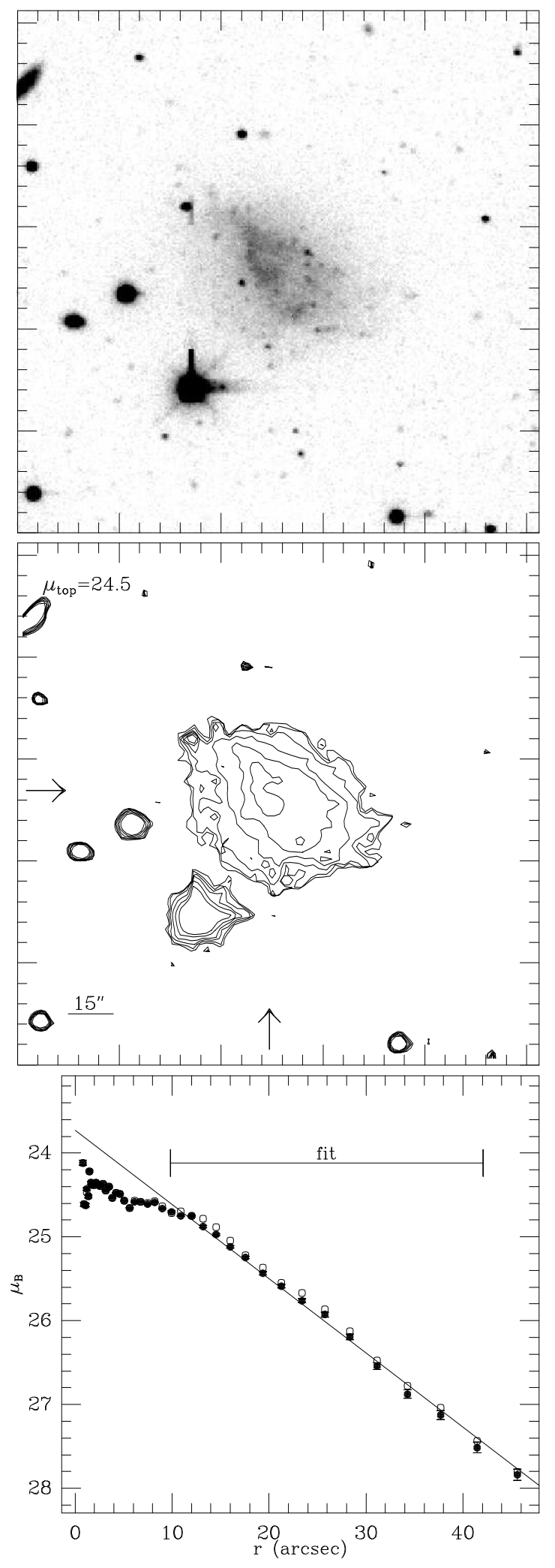
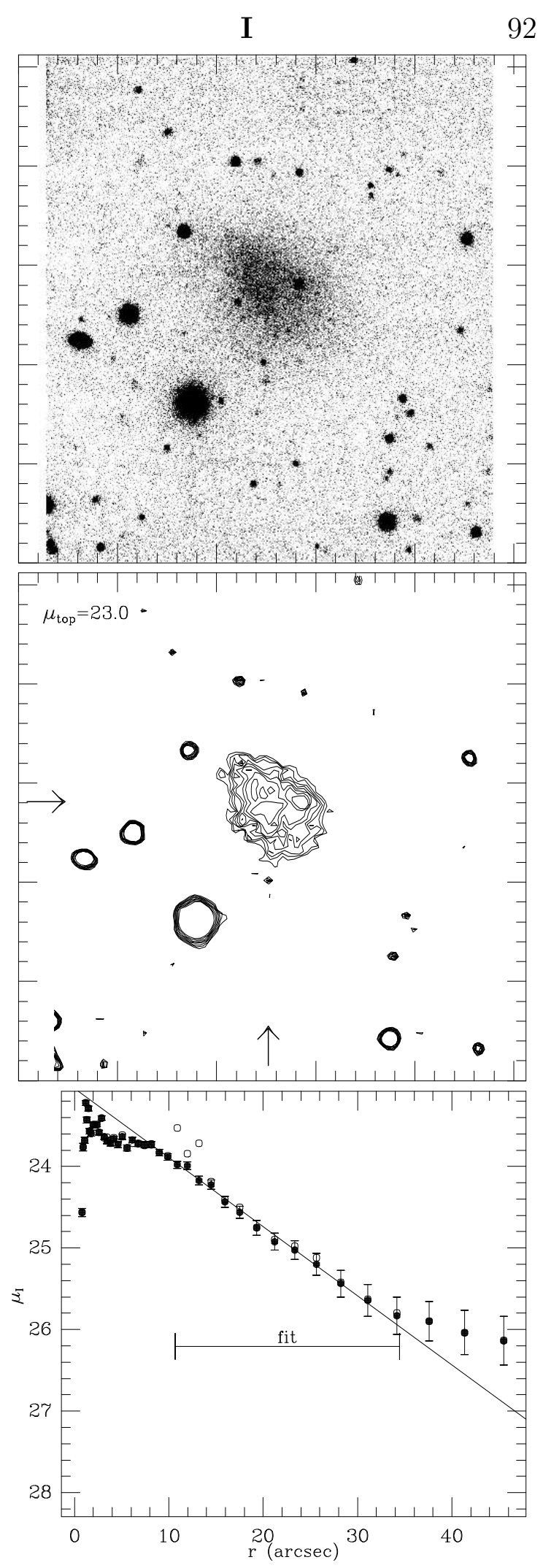

Figure 53: UGC 10669 (B-May85) 
B

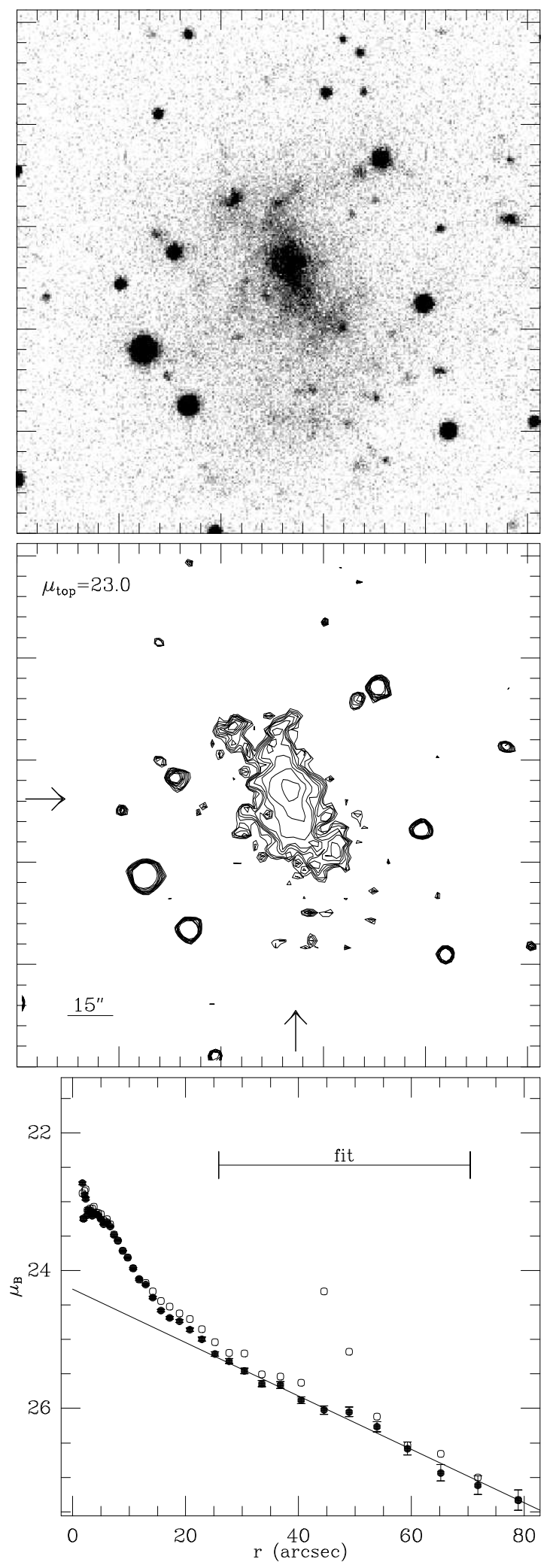

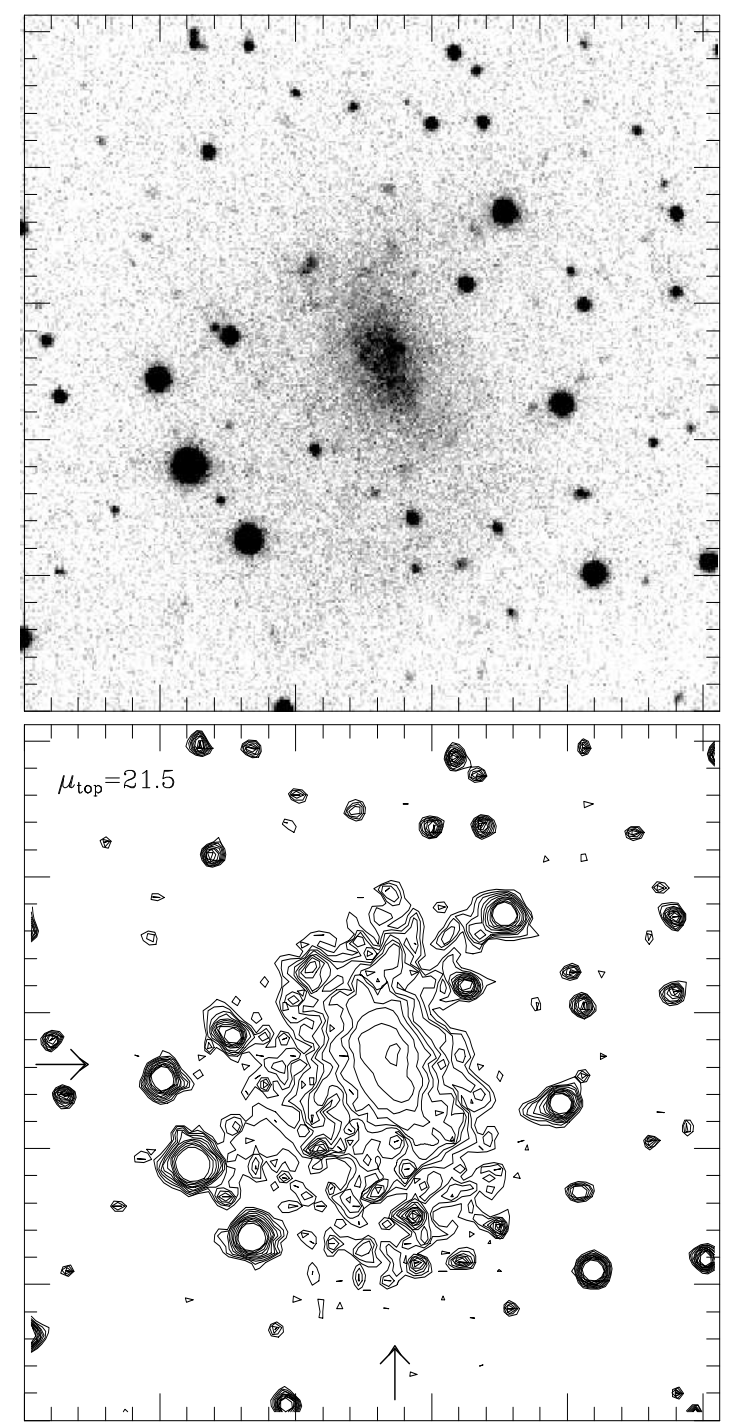

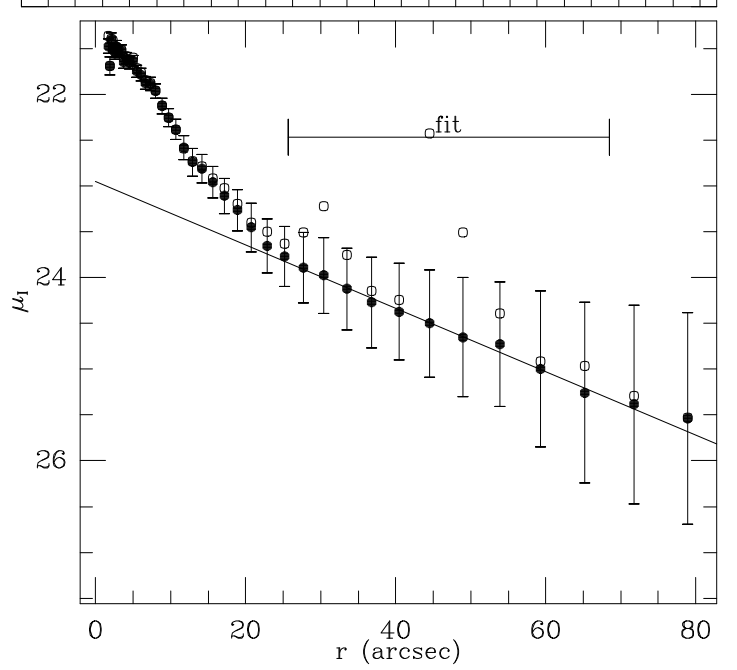

Figure 54: UGC 12082 (Sep91) 
B
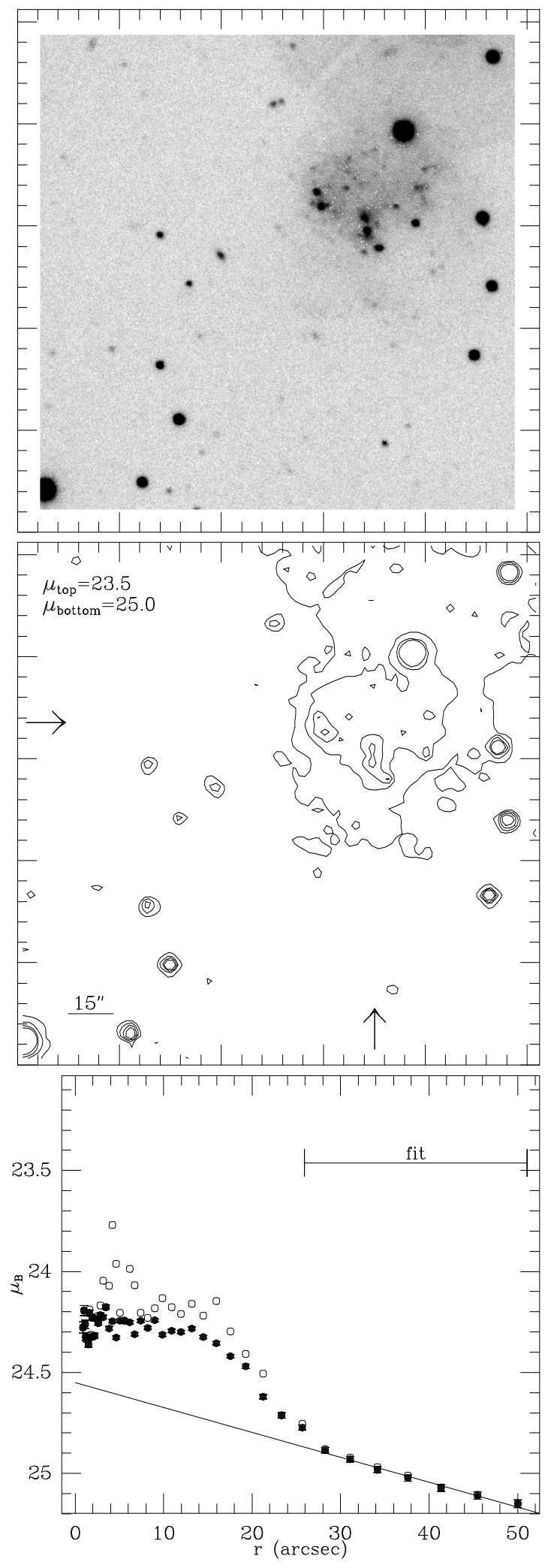

I
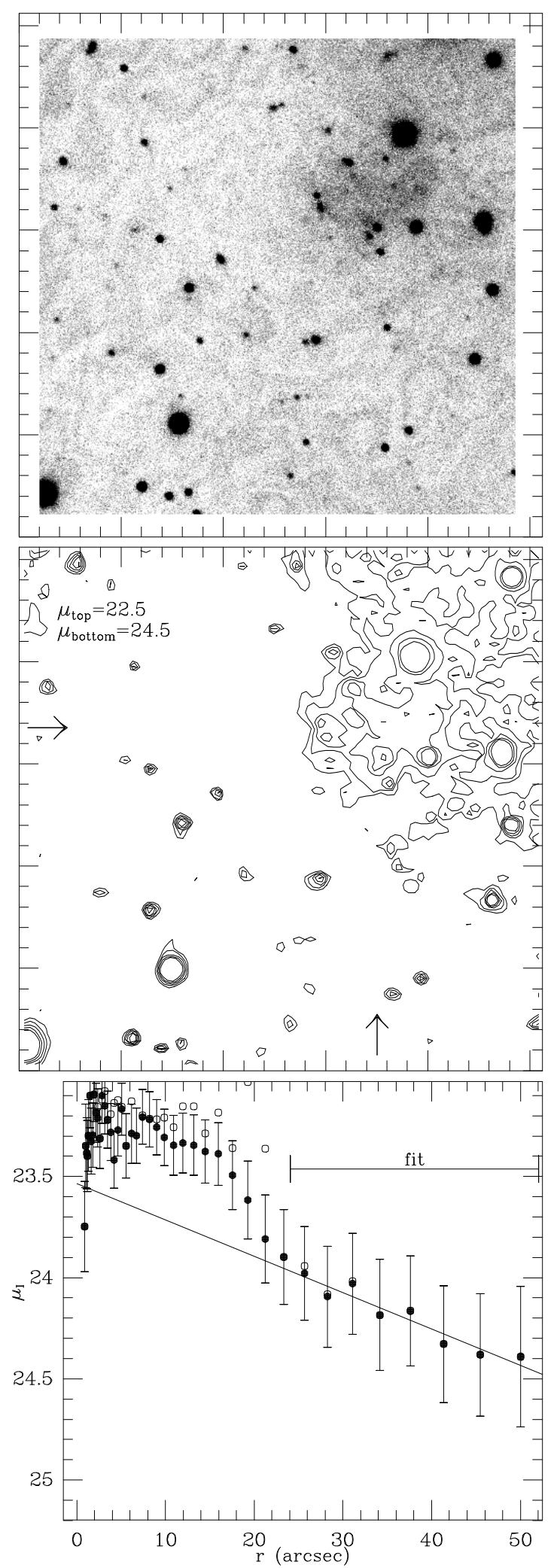

Figure 55: UGC 12894 

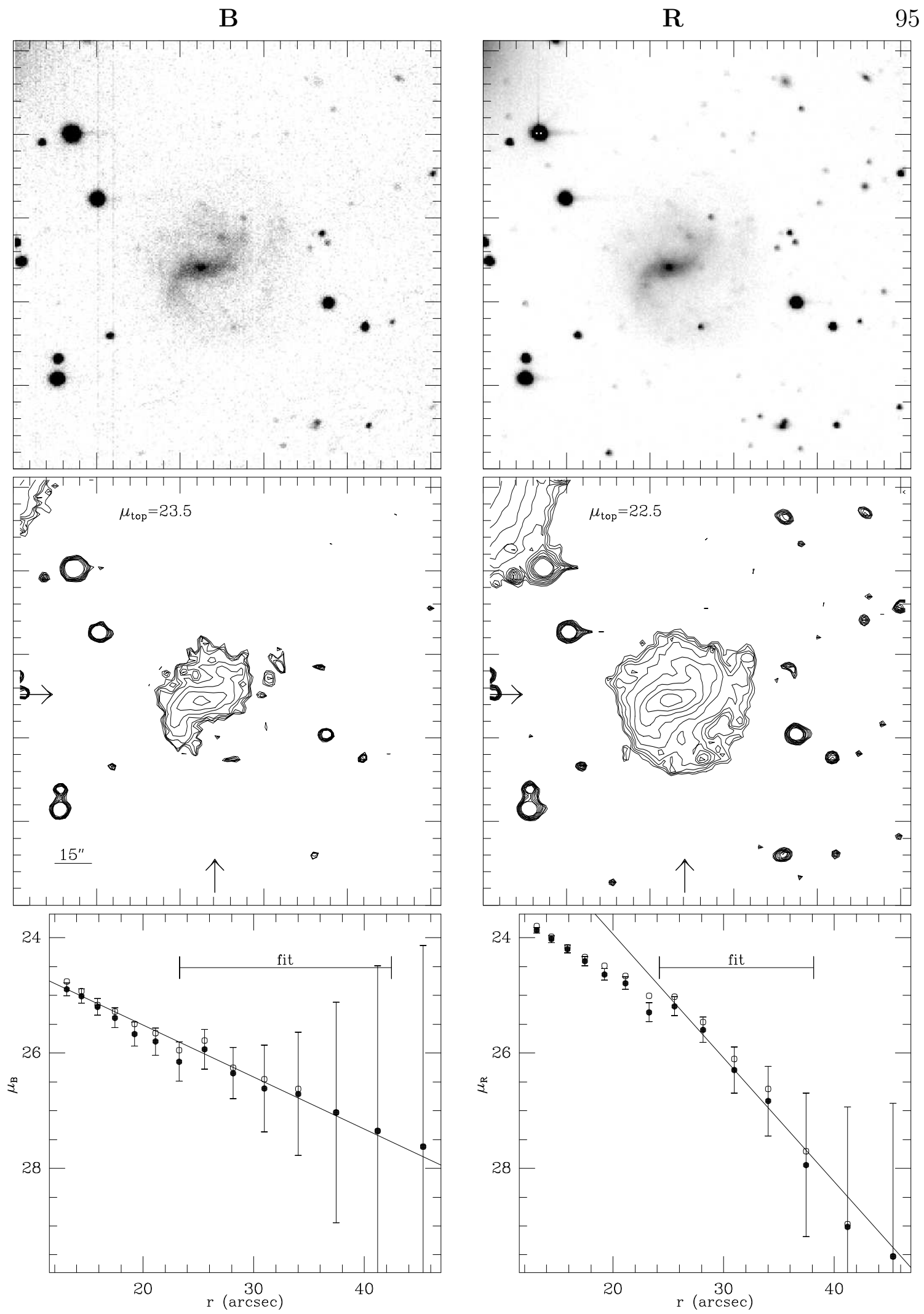

Figure 56: UGC 11764 (May85) 

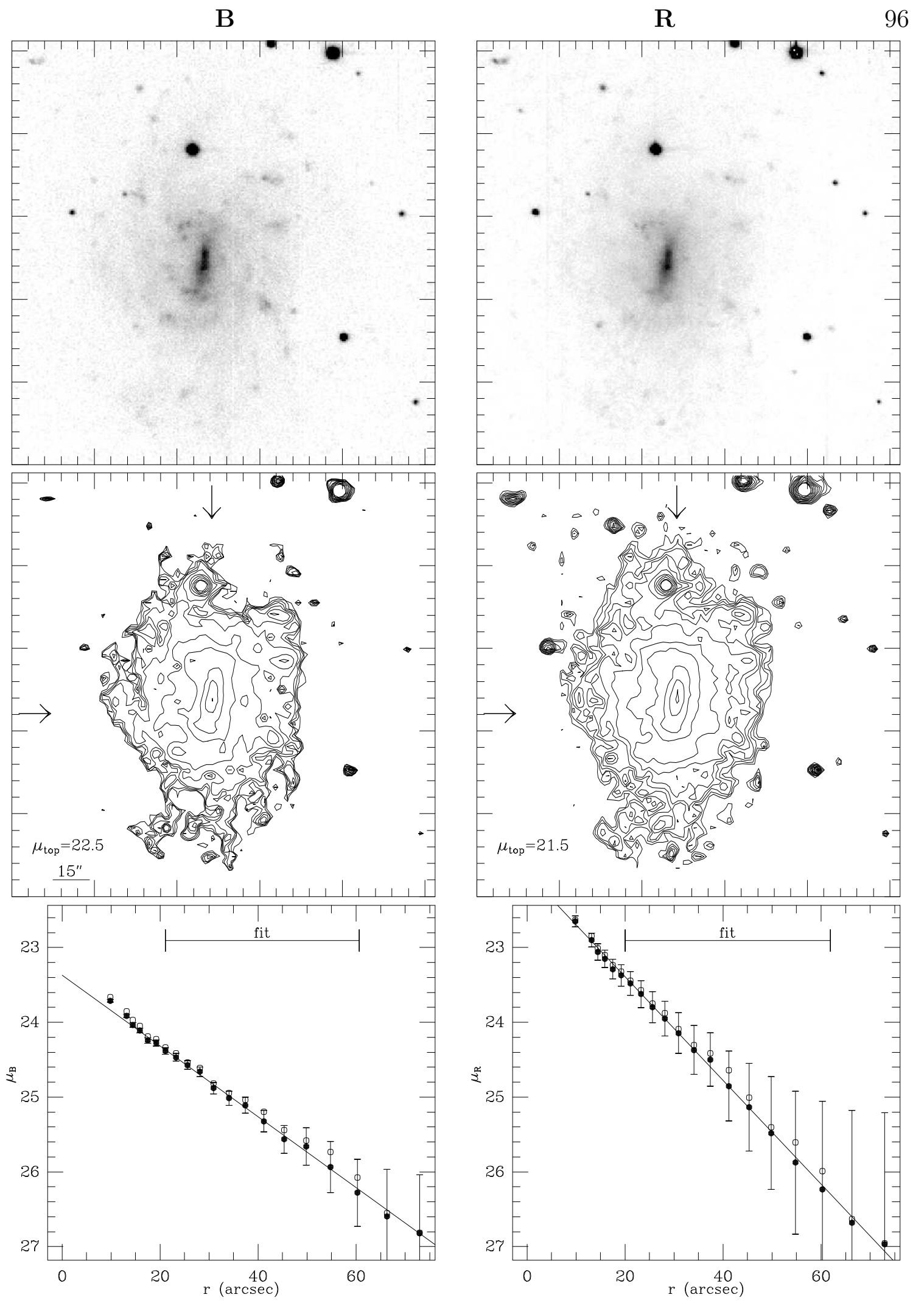

Figure 57: UGC 12151 (May85) 
B

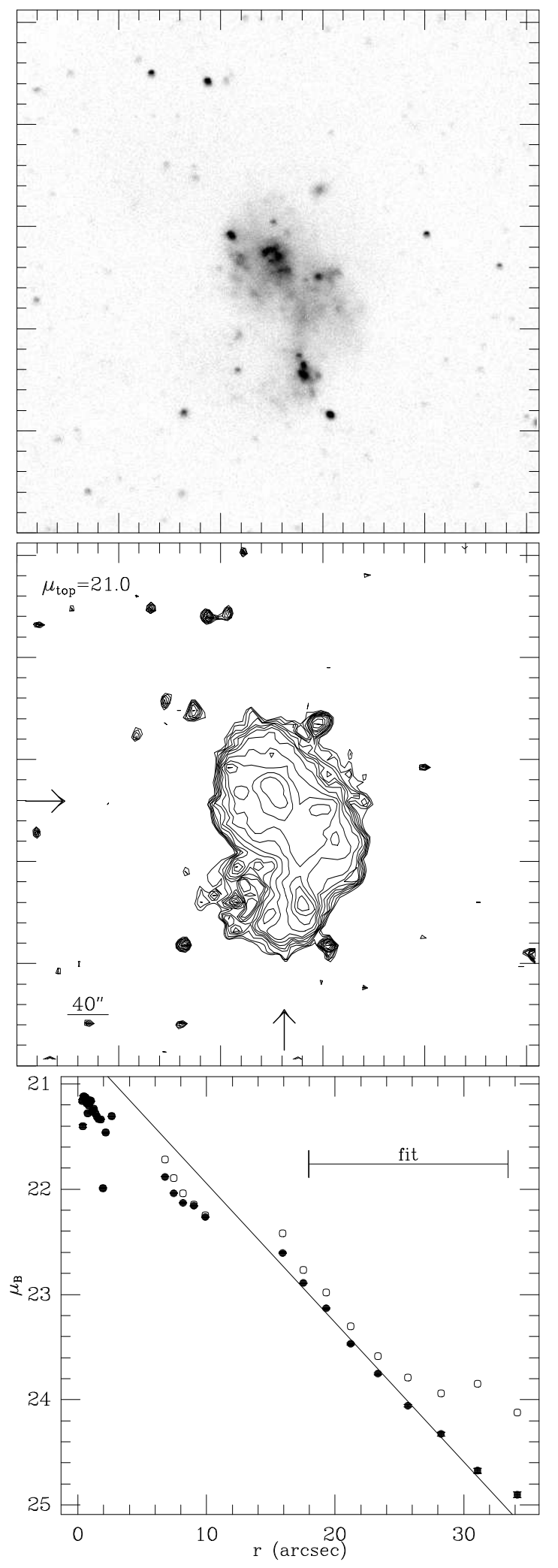

B

97
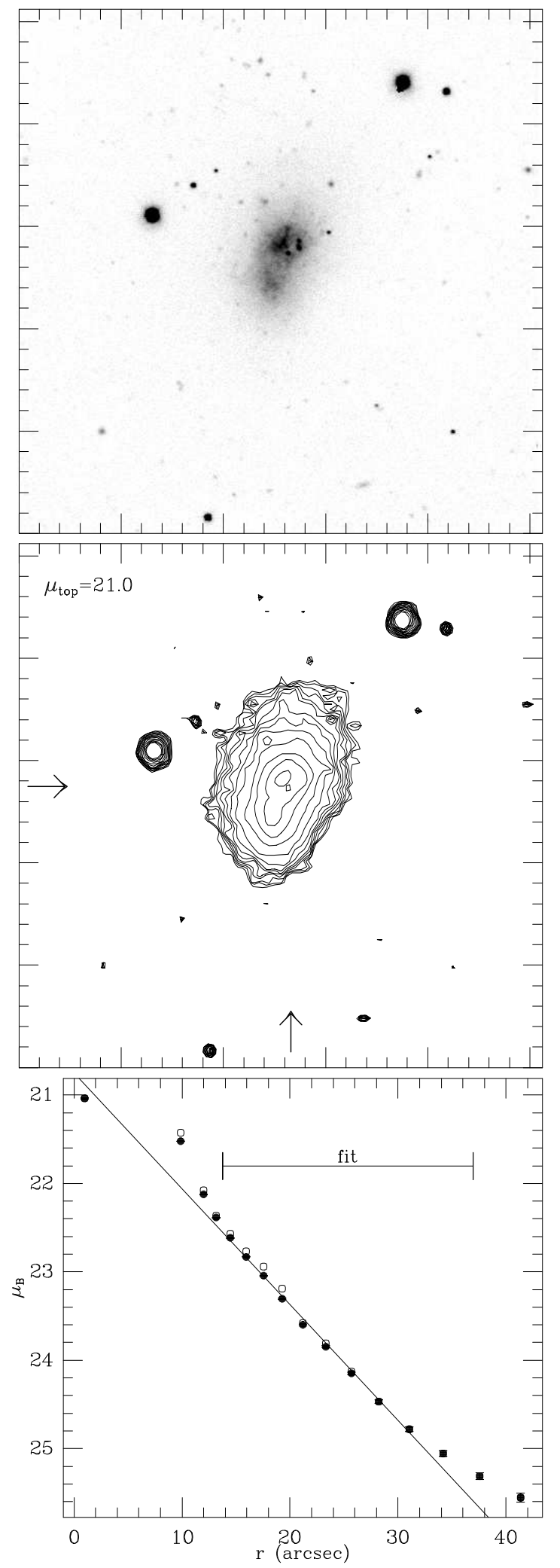

Figure 58: UGC 00772 and UGC 01171 
B

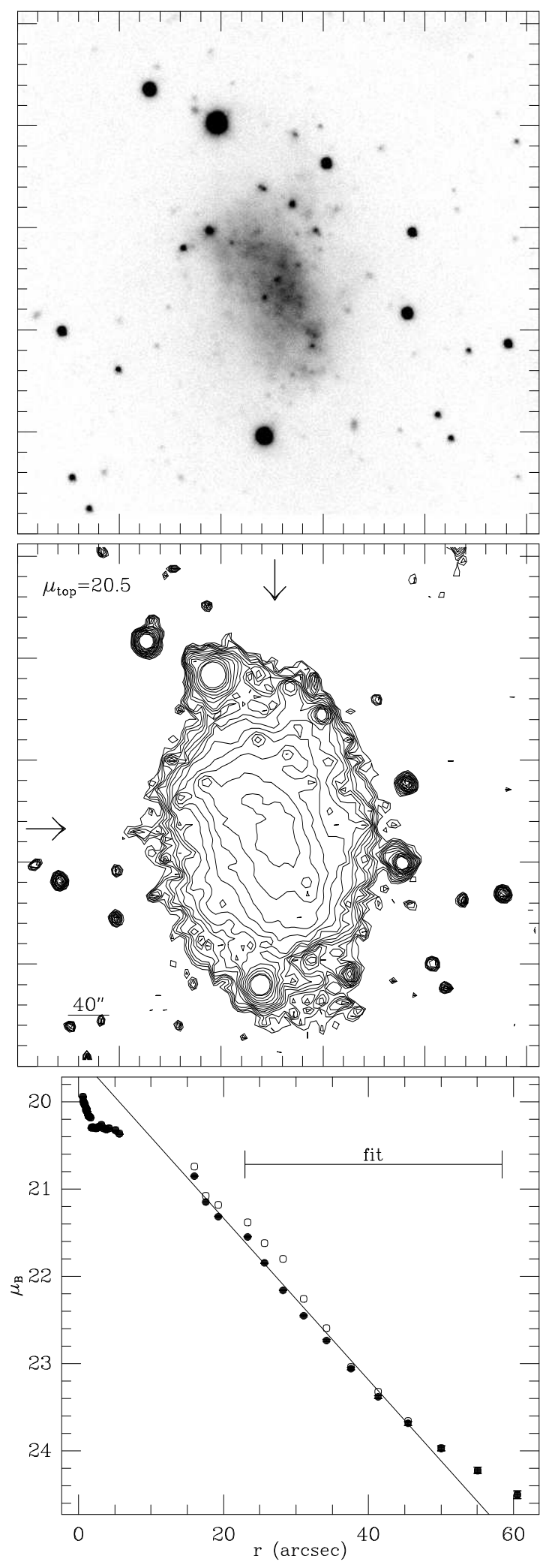

$\begin{array}{rr}\text { B } & 98\end{array}$
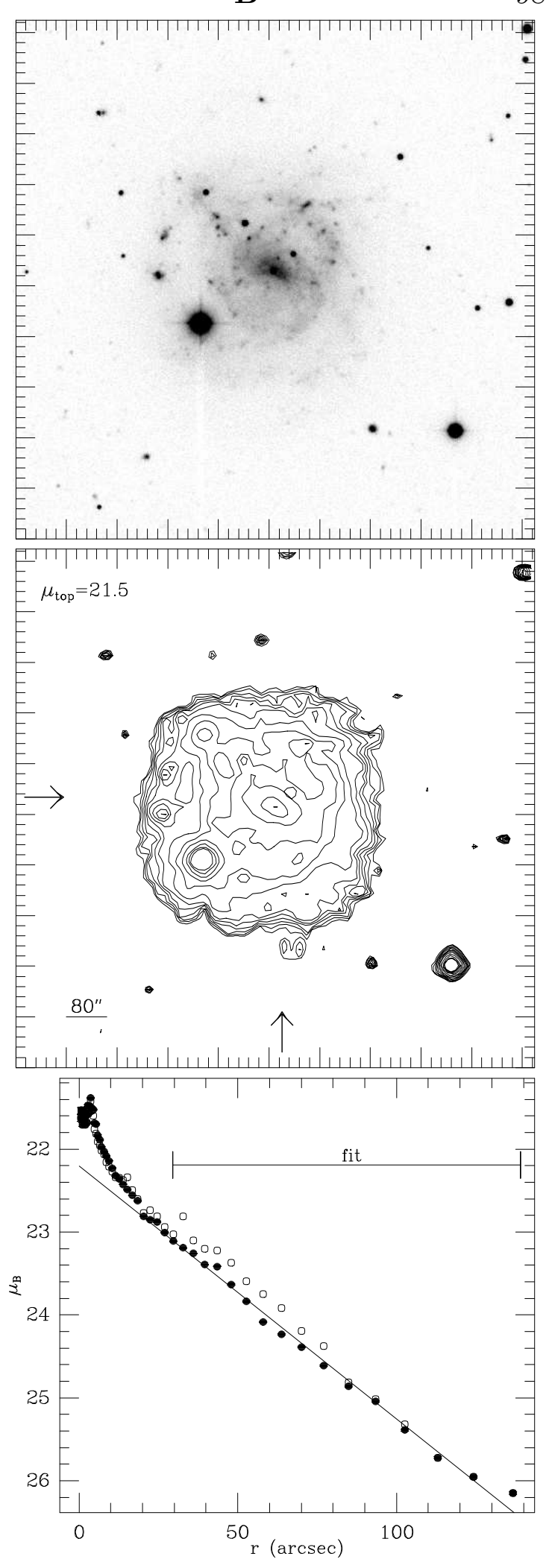

Figure 59: UGC 03860 (Oct90) and UGC 06628 (Apr93) 
B
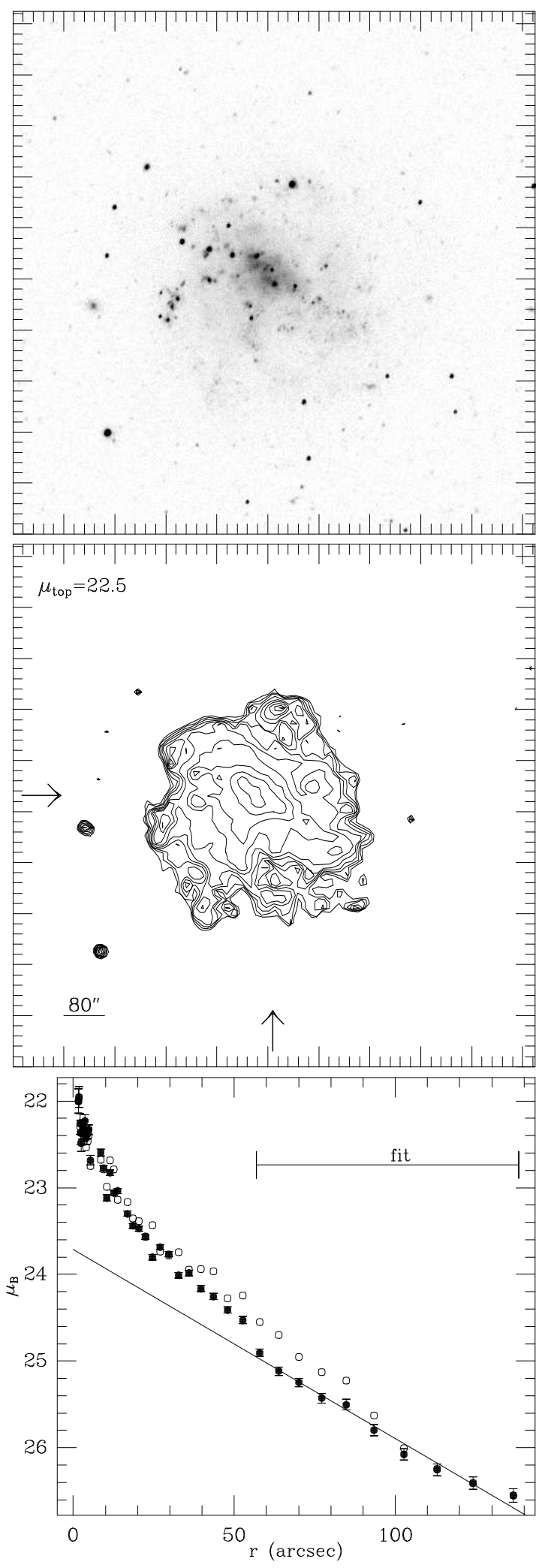

B
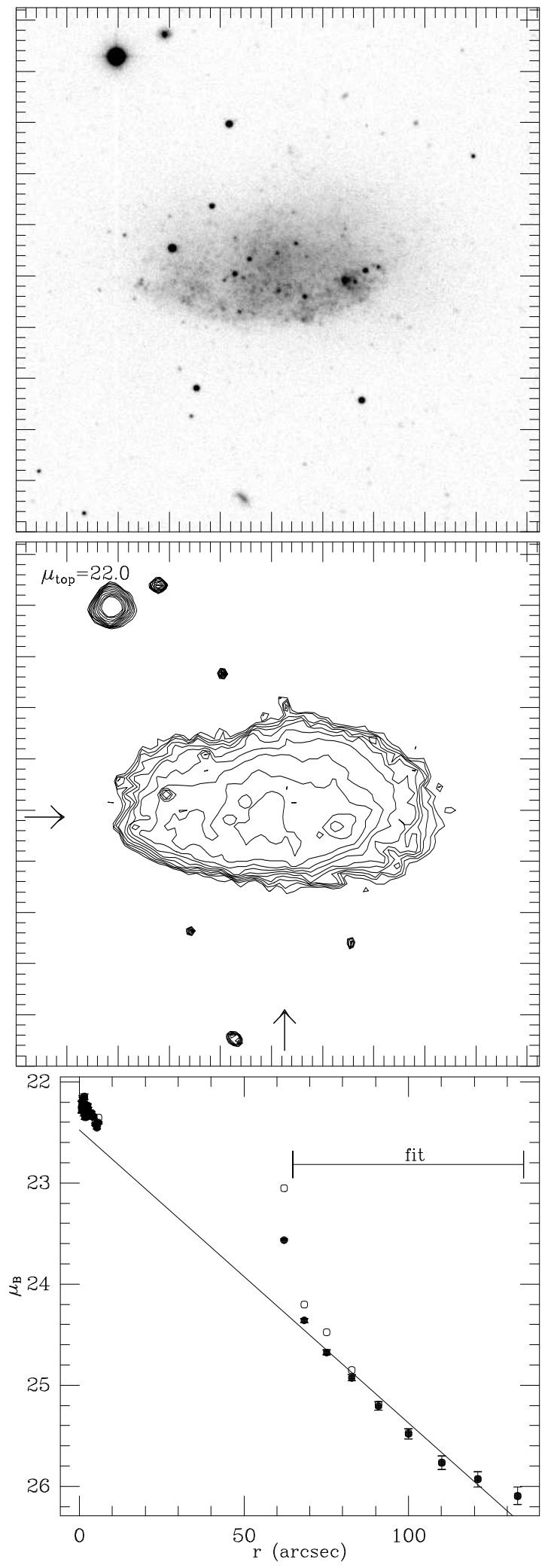

Figure 60: UGC 07608 and UGC 08201 (both Apr93) 
$\mathbf{R}$

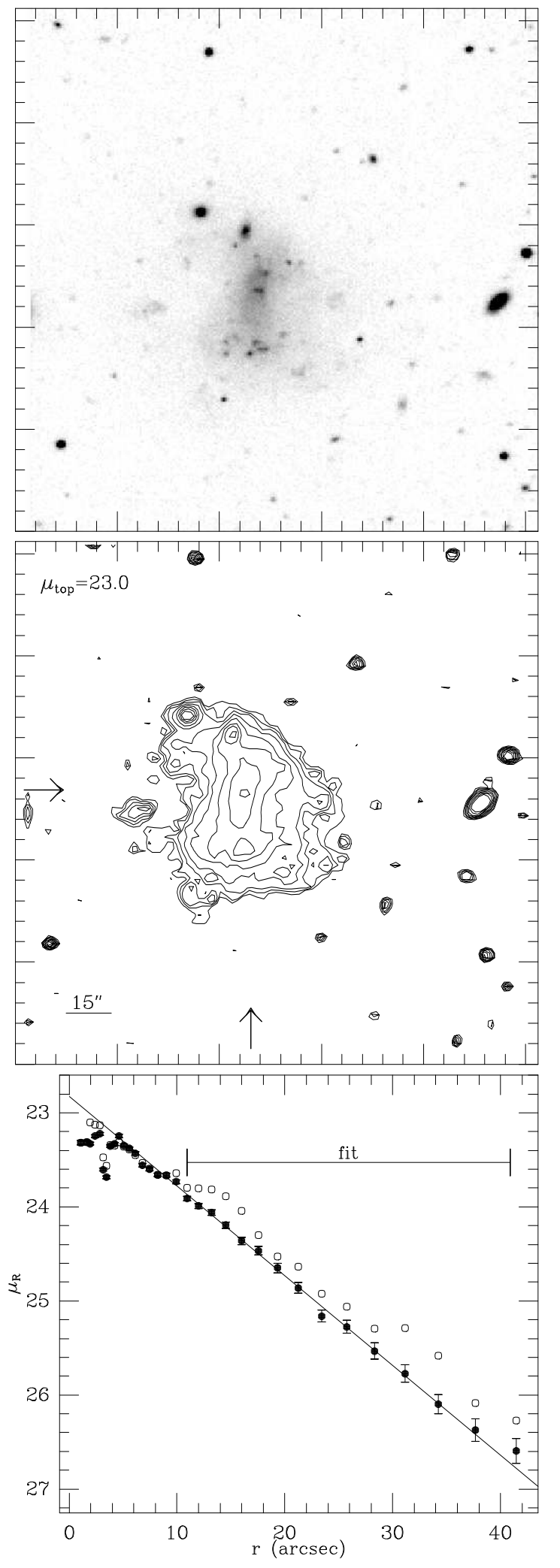

R $\quad 100$
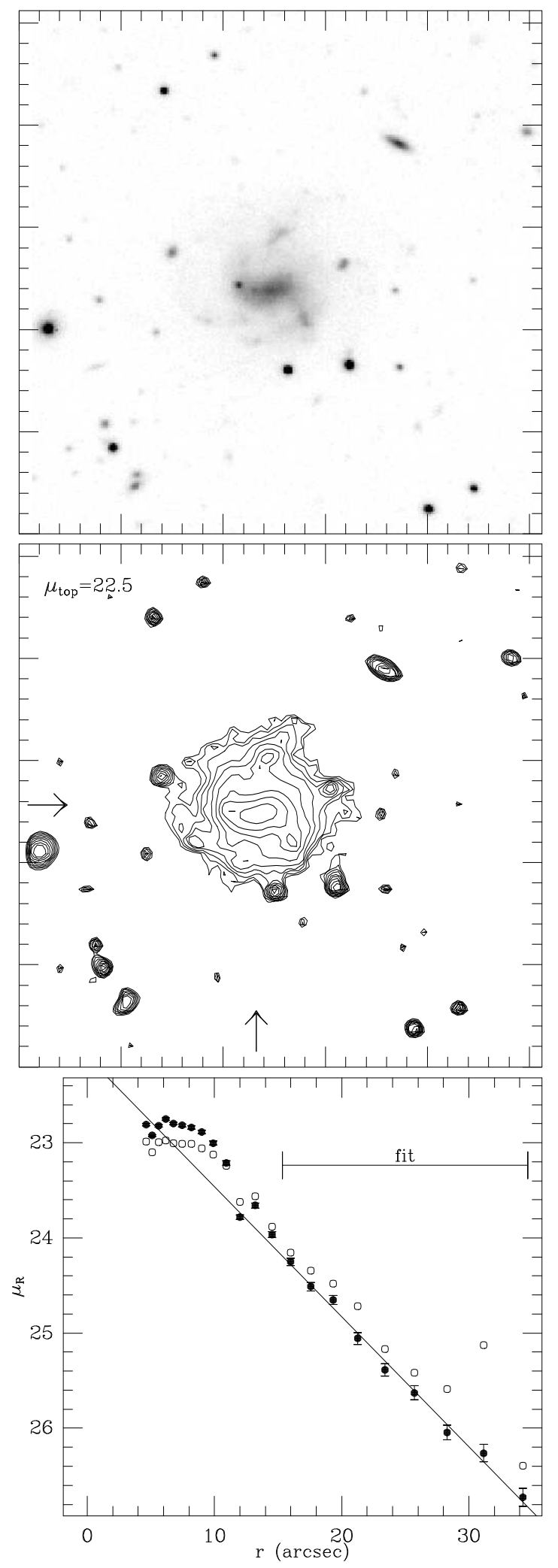

Figure 61: UGC 10031 and UGC 10058 (both May85) 
$\mathbf{R}$

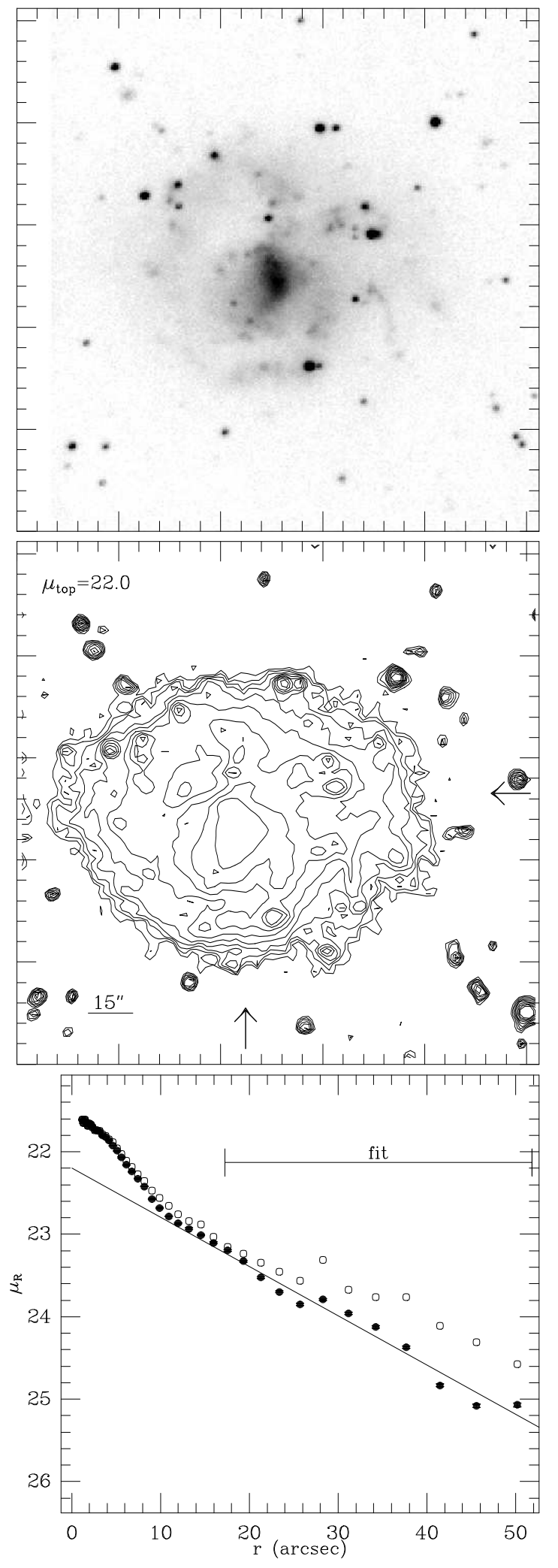

$\mathbf{R}$

101
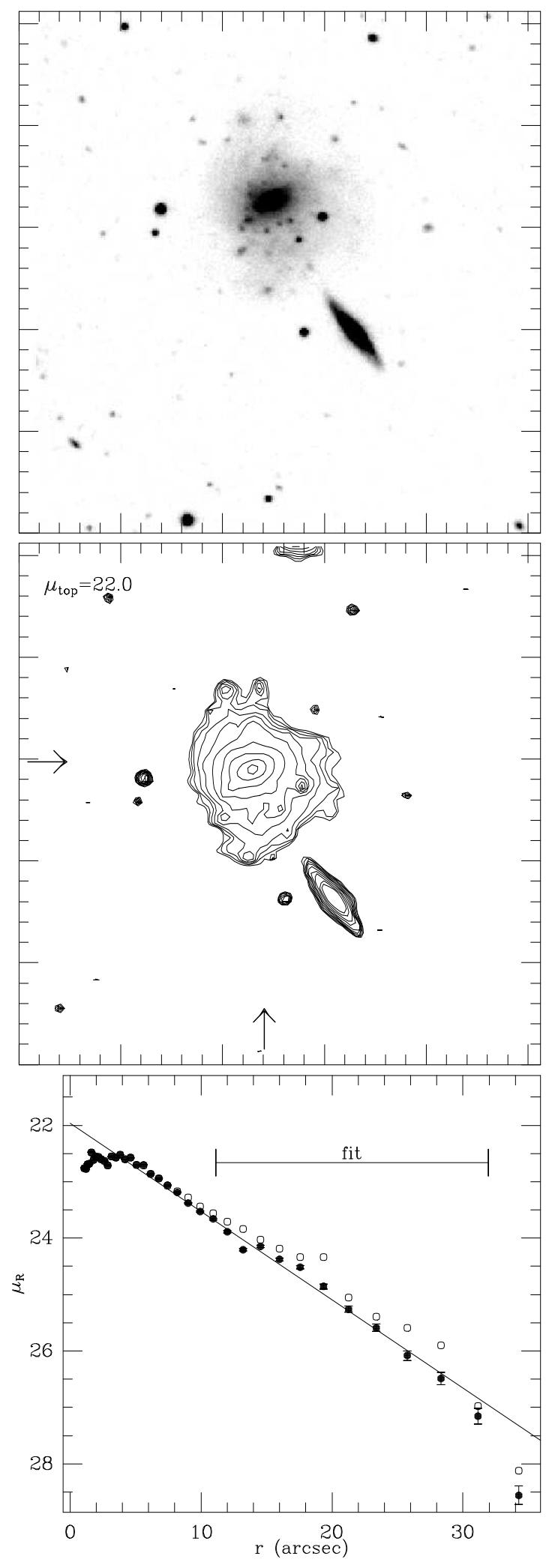

Figure 62: UGC 10290 and UGC 10376 (both May85) 


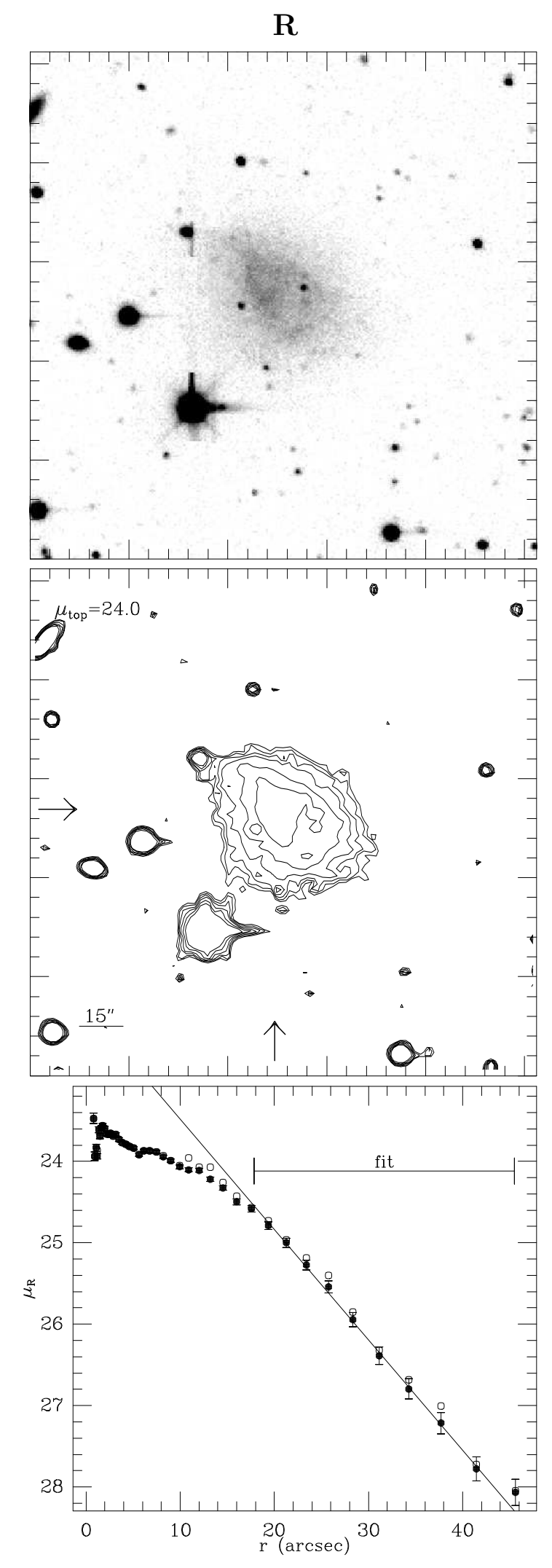

Figure 63: UGC 10669 (May85) 
Figures 64-71: Ellipticity, $\operatorname{Cos}(4 \theta)$ coefficient, and Color Profiles of all Galaxies in the Sample.

In the upper panel, the ellipticity, $\epsilon$ is plotted as a function of effective radius for each galaxy. Here $\epsilon=1-(b / a)$, where $b / a$ is the ratio of the semi-minor to semi major axis found from the I-band image (except when not available) using the GALPIIOT routines. The ellipticity tends to converge to a stable value as $r$ increases. This is the value that was adopted in order to obtain the inclination, as described in Chapter 3 .

The middle panel contains plots of the $\cos (4 \theta)$ term (or $A_{4}$ parameter) as a function of effective radius. This parameter is commonly used in studies of elliptical galaxies to measure the deviation from ellipticity of the isophotes. In this case, we use the parameter to obtain a measure of the applicability of elliptical fitting. Large deviations from 0 of the $A_{4}$ parameter indicate that the isophotes were poorly fit by ellipses.

The $B-I$ color profiles are plotted as a function of effective radius in the lower pancl. The color profiles were obtained from the differenec of the surface brightness measured within each elliptical annulus (again, with the ellipses determined from the $I$-band image). The surface brightness used here is the surface brightness excluding the HII regions and any stars present. For the seven galaxies for which $h$-band data also exist, the $B-R$ profiles are plotted with open circles. In the case of the 5 galaxies for which subsequent $I$-band data was obtained, the $B-I$ color appears to be anomalously red. 'l'his was thought to be caused by poorly determined transformation coeflicients for the May 85 observing rum. 

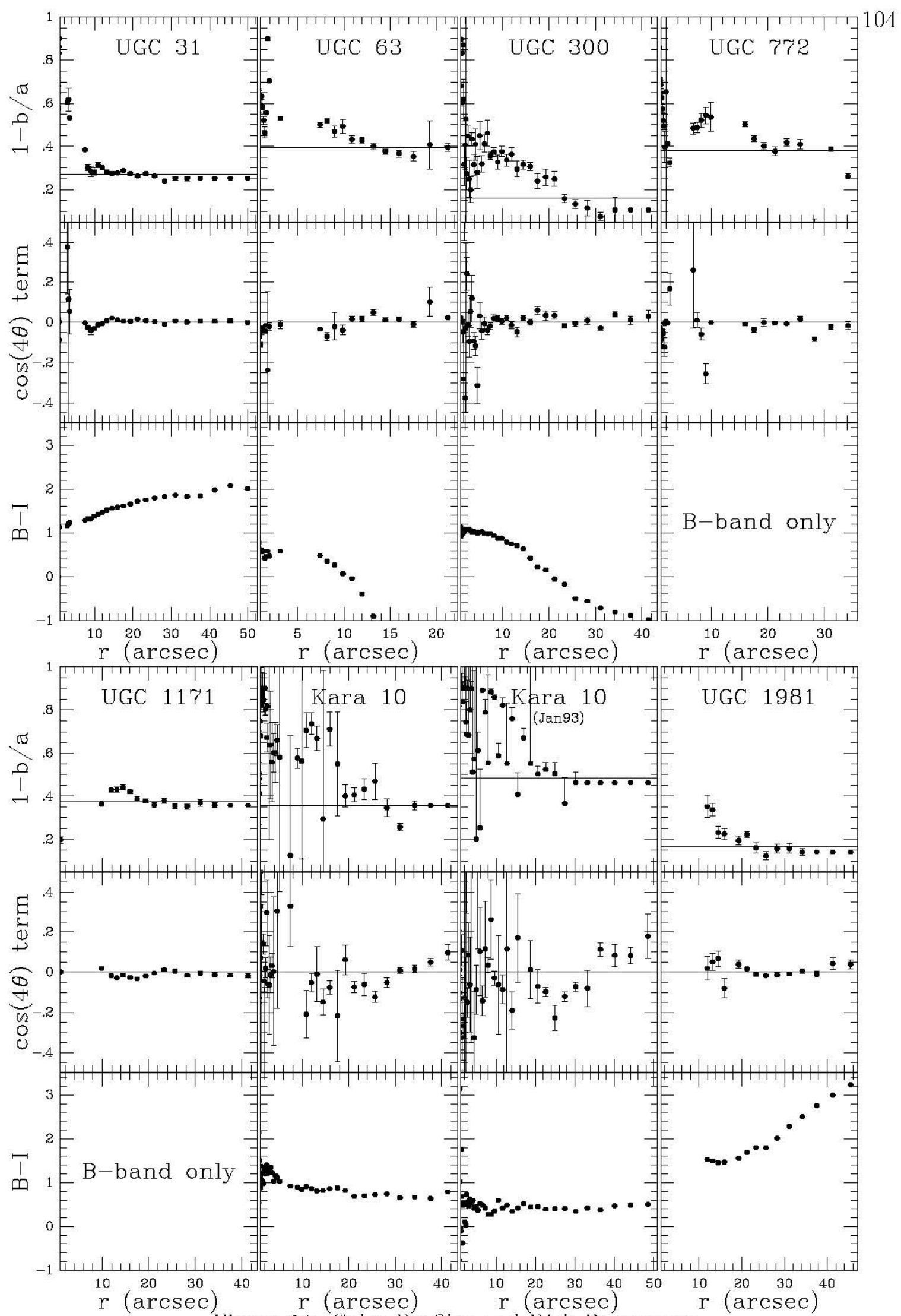

Figure 61: (Color Profiles and 1)isk Parameters 

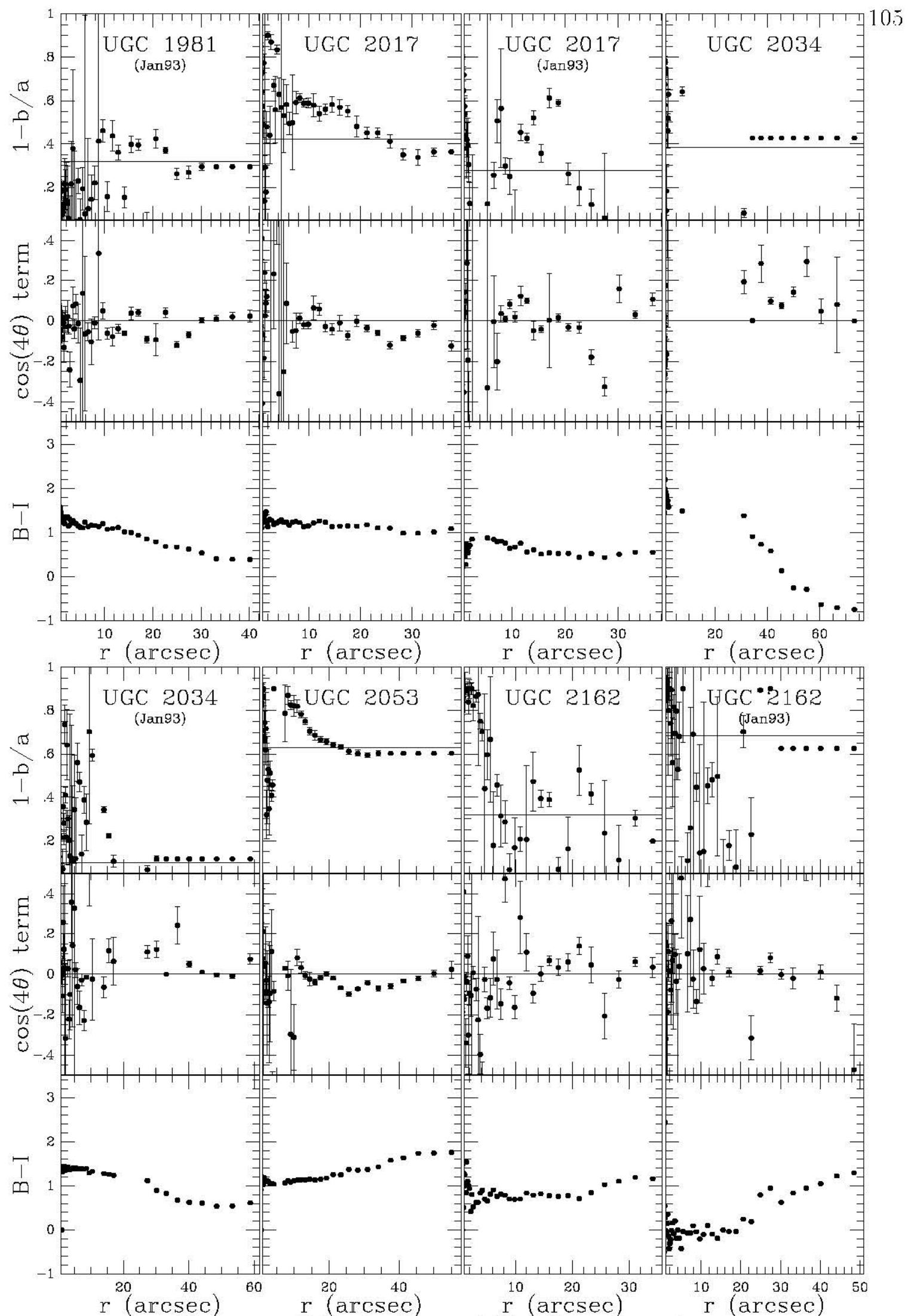

Figure 65: Color Profiles and I)isk Parameters (cont.) 

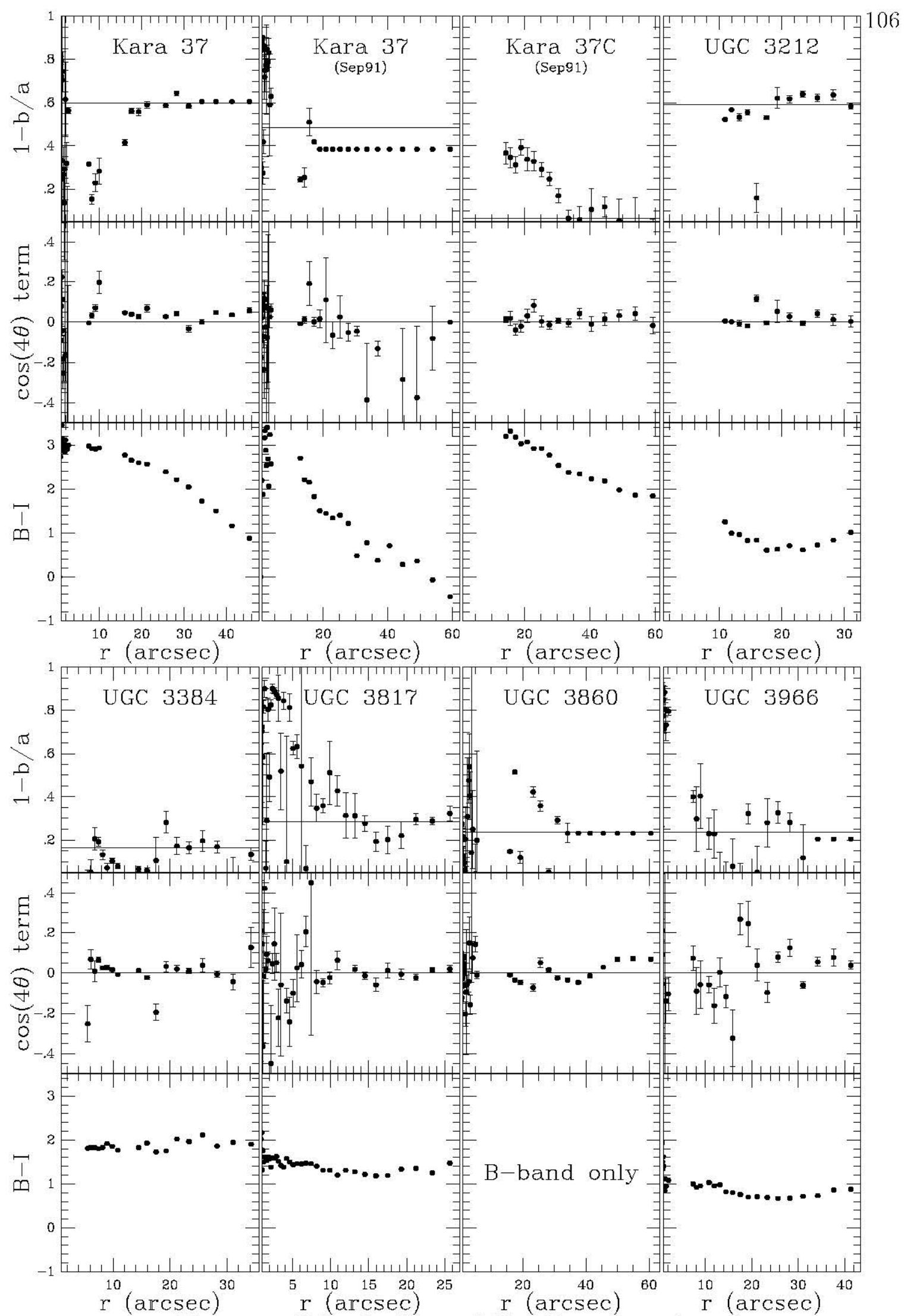

Figure 66: Color Profiles and I)isk Parameters (cont.) 

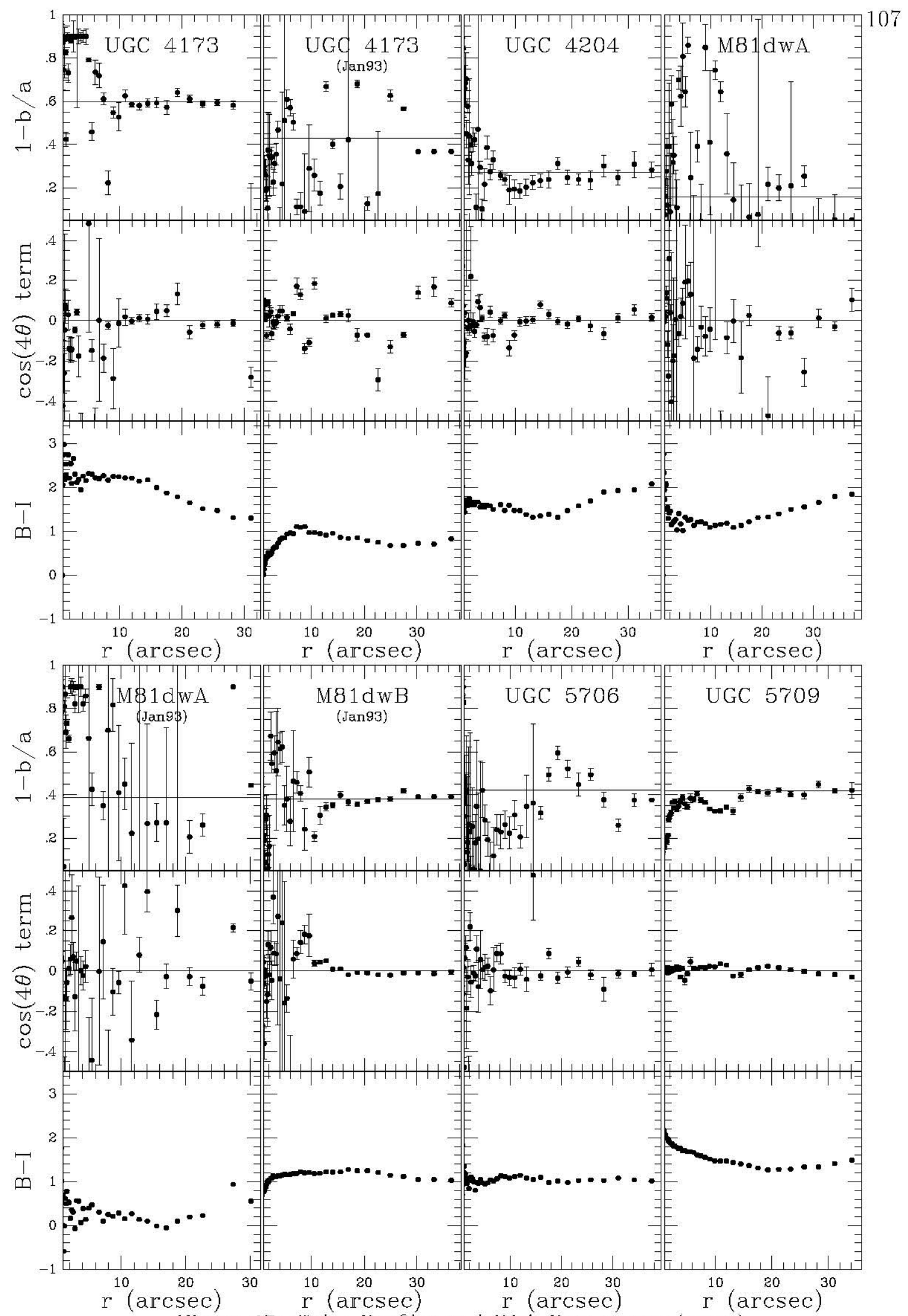

Figure 67: Color Profiles and I)isk Parameters (cont.) 

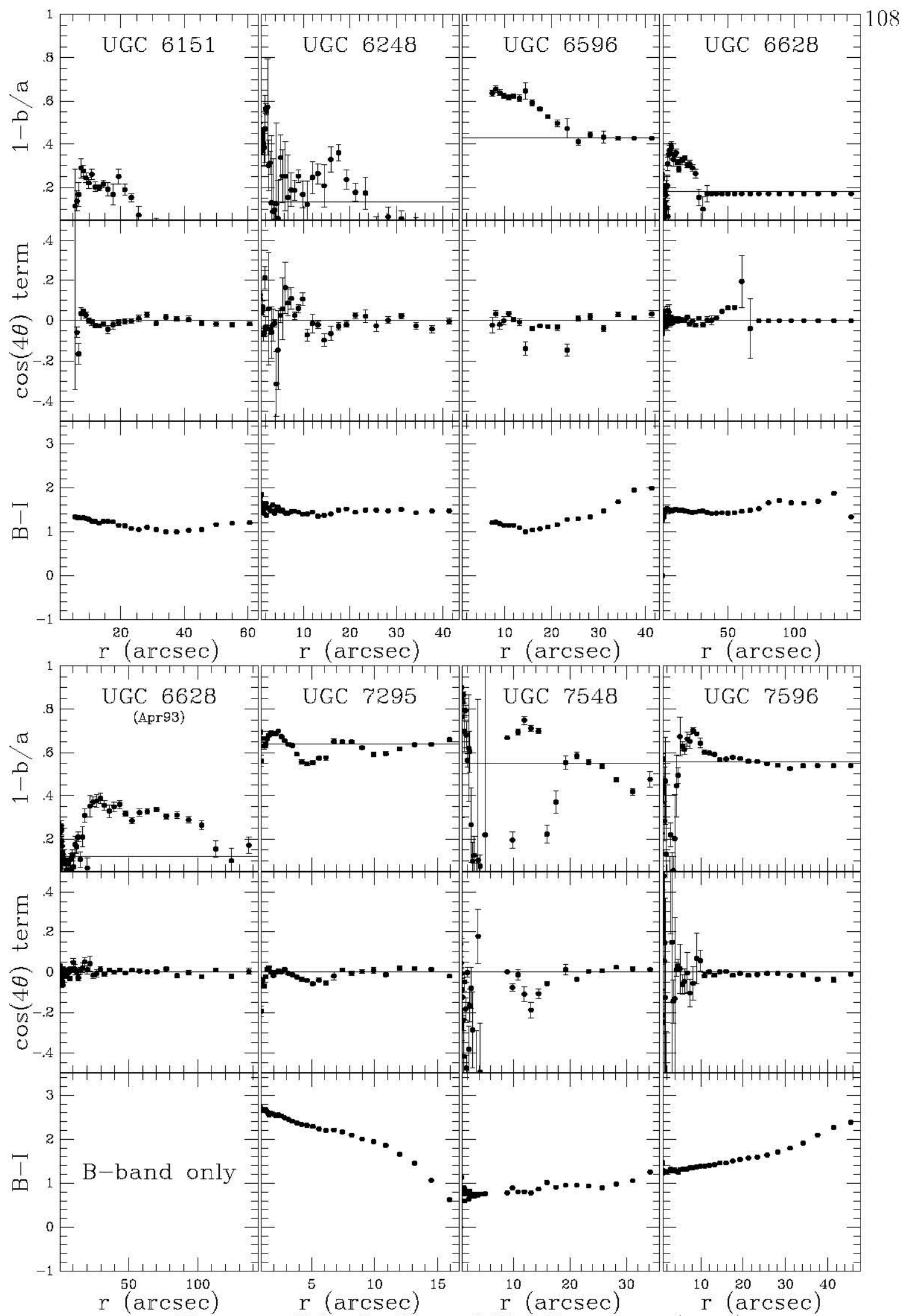

Figure 68: Color Profiles and I)isk Parameters (cont.) 

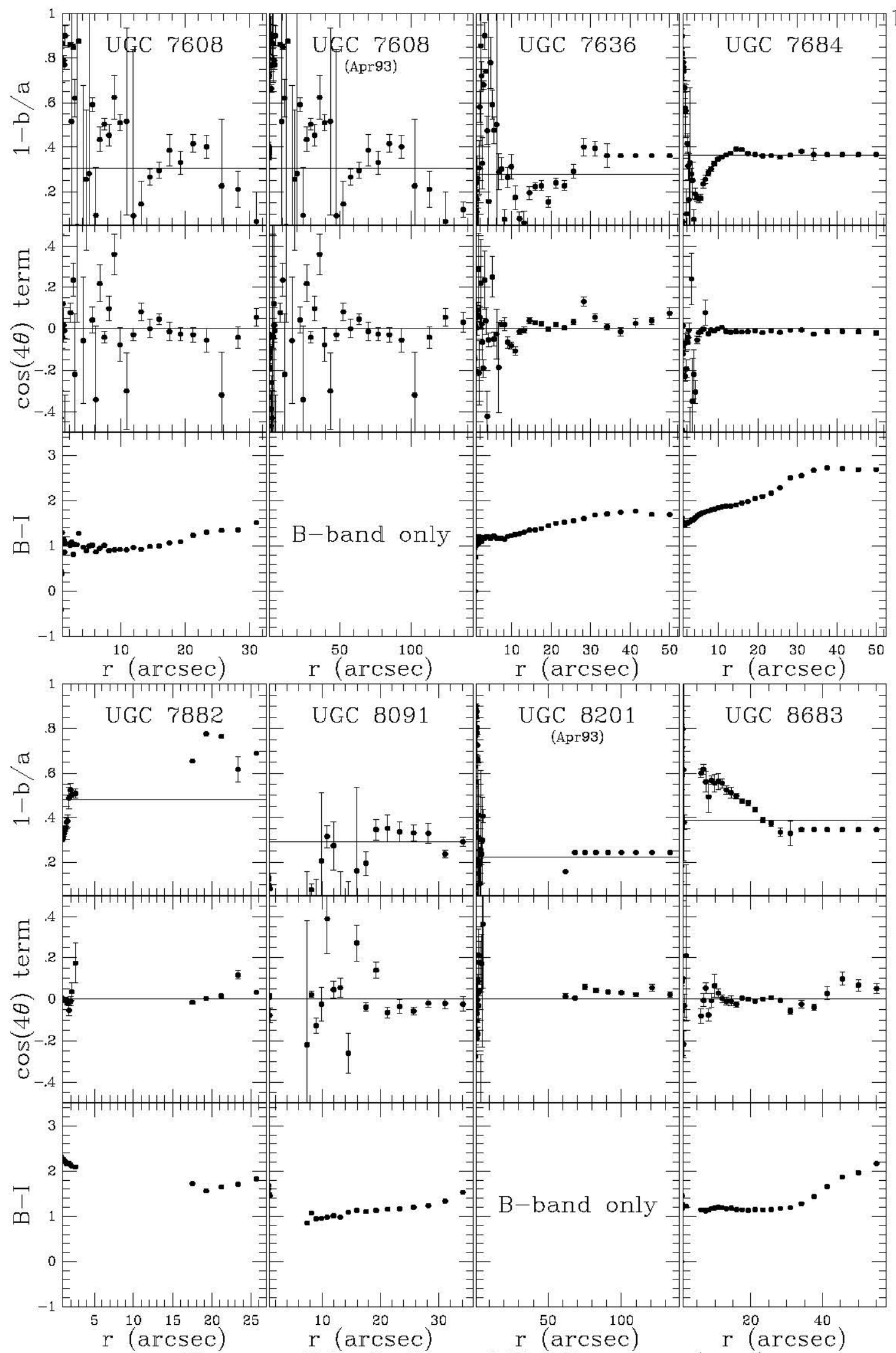

Figure 69: Color Profiles and I)isk Parameters (cont.) 

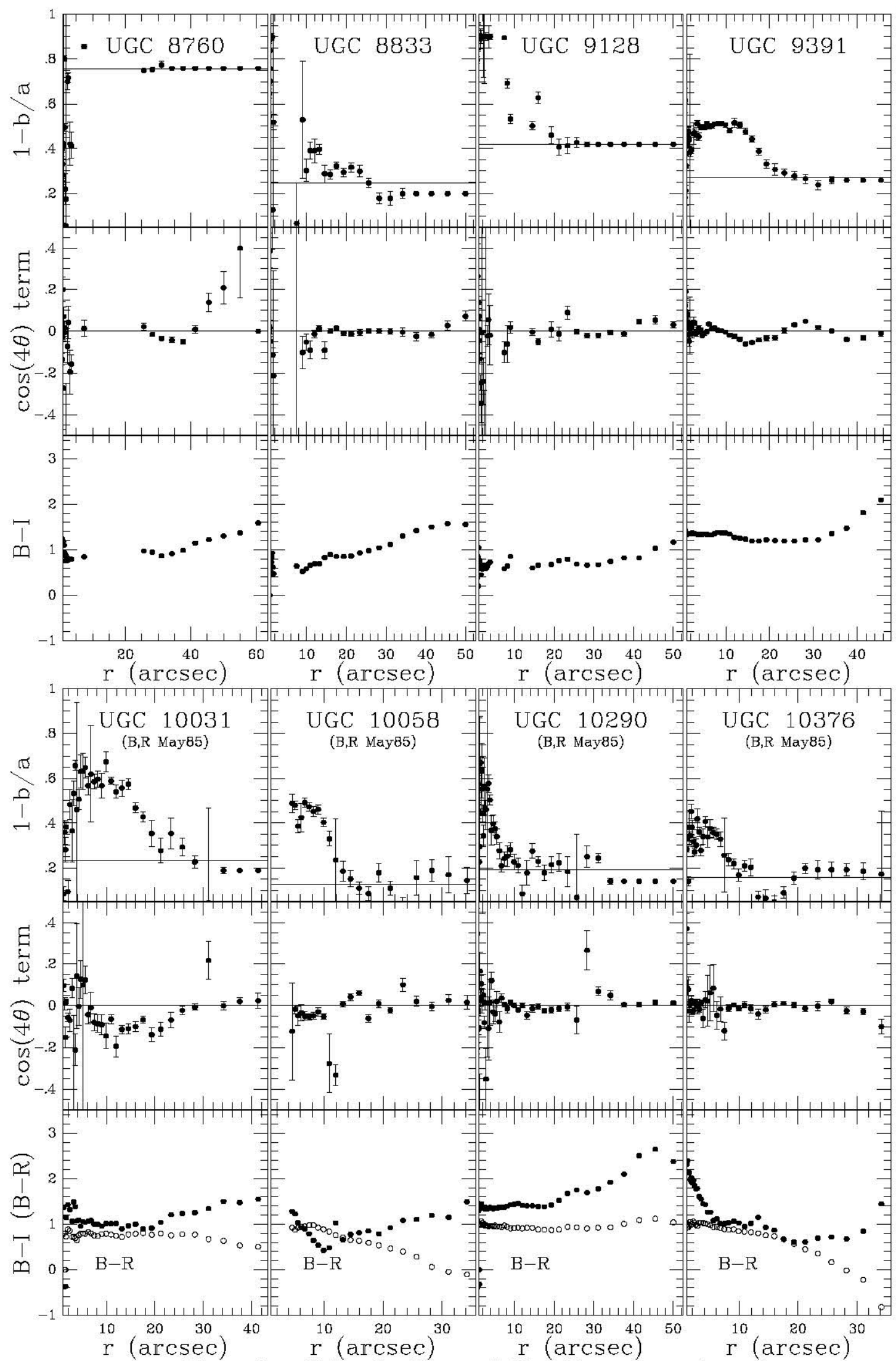

Figure 70: Color Profiles and I)isk Parameters (cont.) 

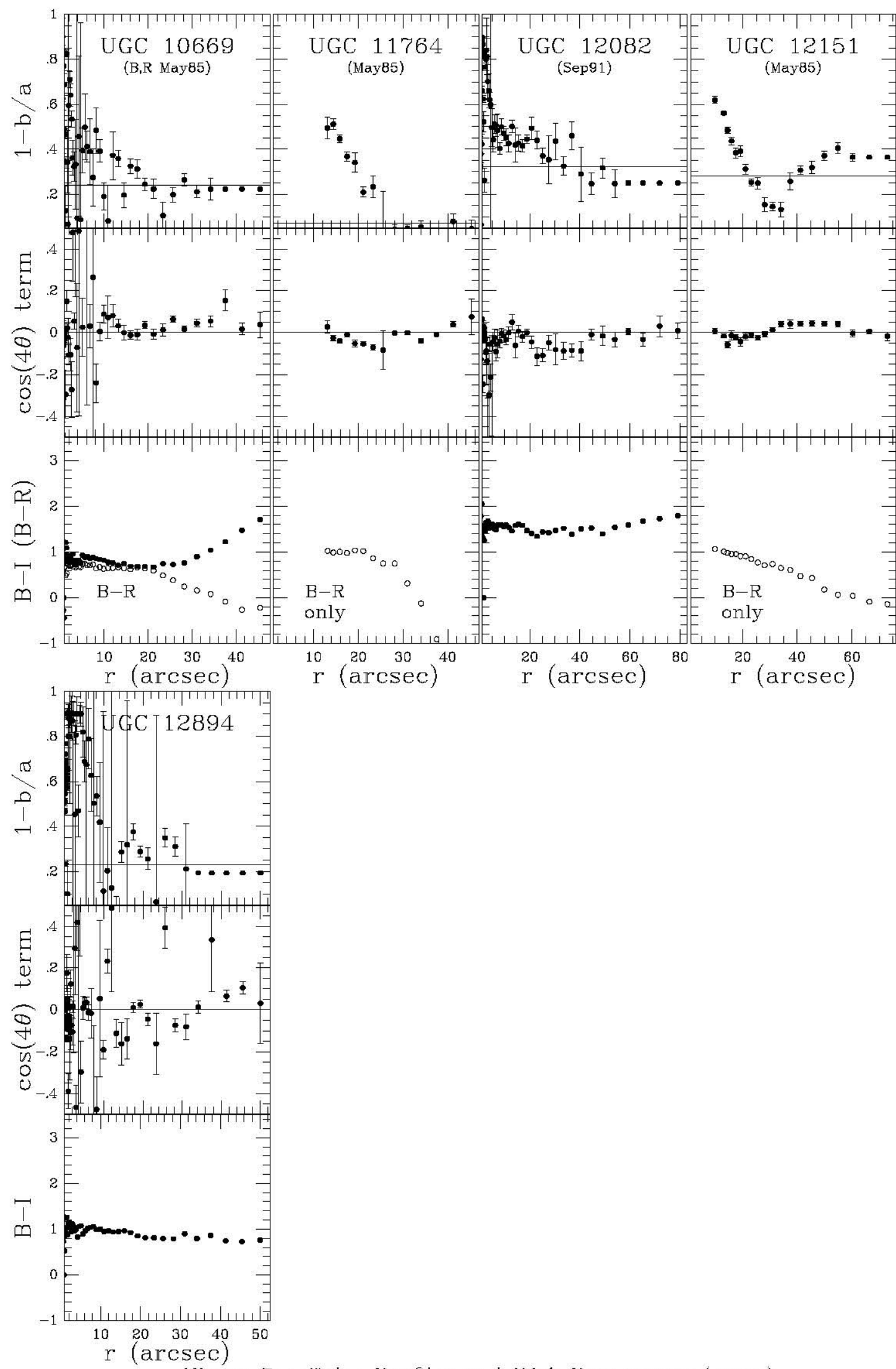

Figure 71: Color Profiles and Disk Parameters (cont.) 


\section{Figures 72-80: Histograms of the Size, Radial, and Azimuthal Dis- tribution of "HII Regions" and Stars for the Galaxies in the Sample.}

Ilistograms showing the size of the regions excluded from the ellipse fitting (as either IIII regions, or stars within the galaxy) are given in Figures 72-74 for each galaxy. The size is in parsecs, based on the distane for cach galaxy listed in Table 7 (Chapter 3). For histograms which extend past the maximum shown in the figure, the actual number is given in parentheses next to the peak. The discrete, "spikey", nature of the distribution is due to the finite pixel size. The radius smallest, and often largest, peak 11sually translates to one pixel on the detector. A histogram showing the distribution for all the galaxies taken as a whole is shown in at the bottom of Figure 74 .

Histograms showing the logarithmic radial distribution of regions from the center of the galaxy are shown in Figures 75-77. Similar histograms presenting the azimuthal distribution (measured counterclockwise from the West) of the regions are given in Figures 78 80. Most galaxics have a fairly smooth azimuthal distribution, though some display peaks which correlate to ridges of star formation. Again, at the end of each set of histograms, there is a figure showing the distribution for all the galaxies taken as a whole (bottom of Figure 77 and Figure 80). 


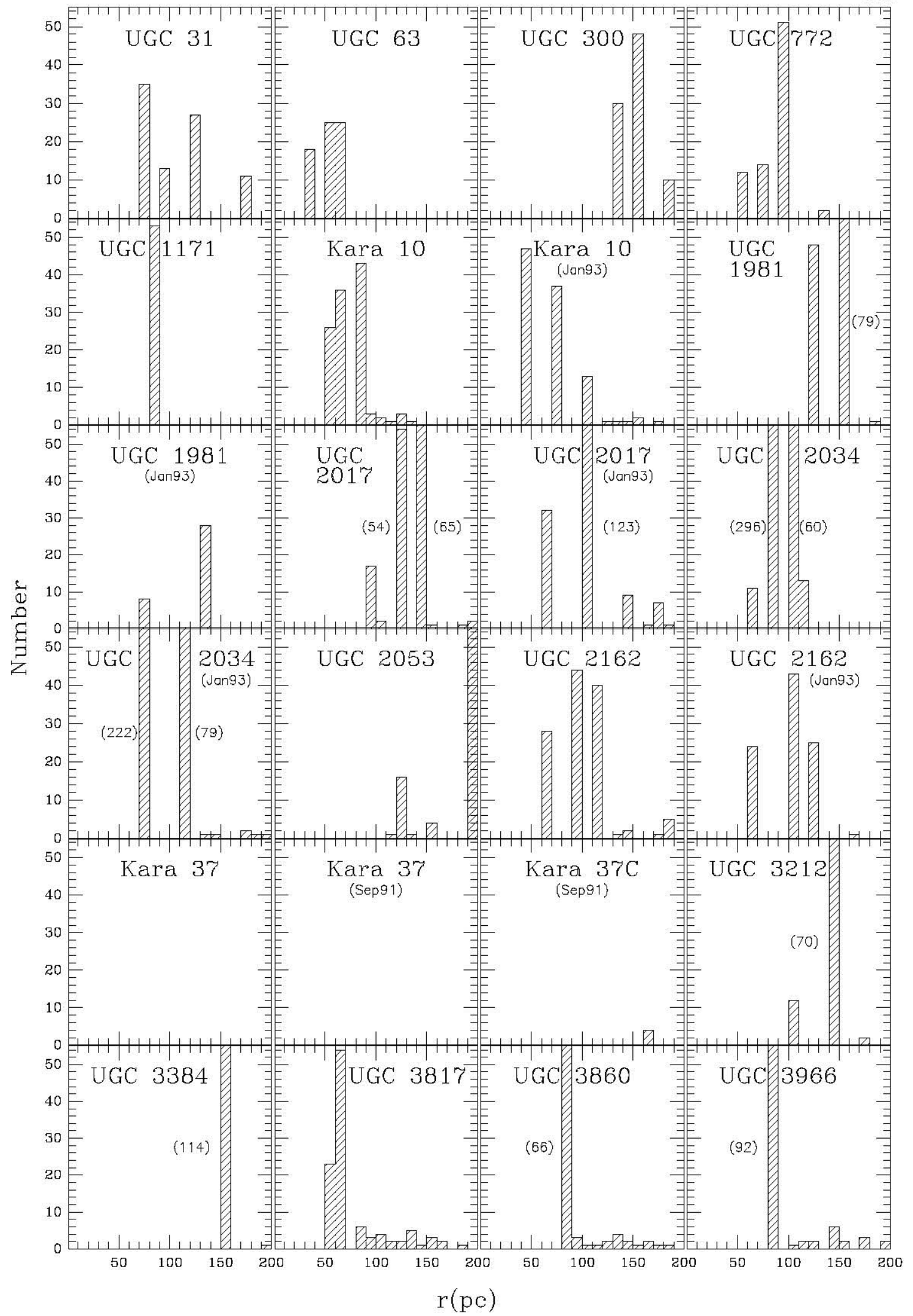

Figure 72: HII Region Size Distribution 


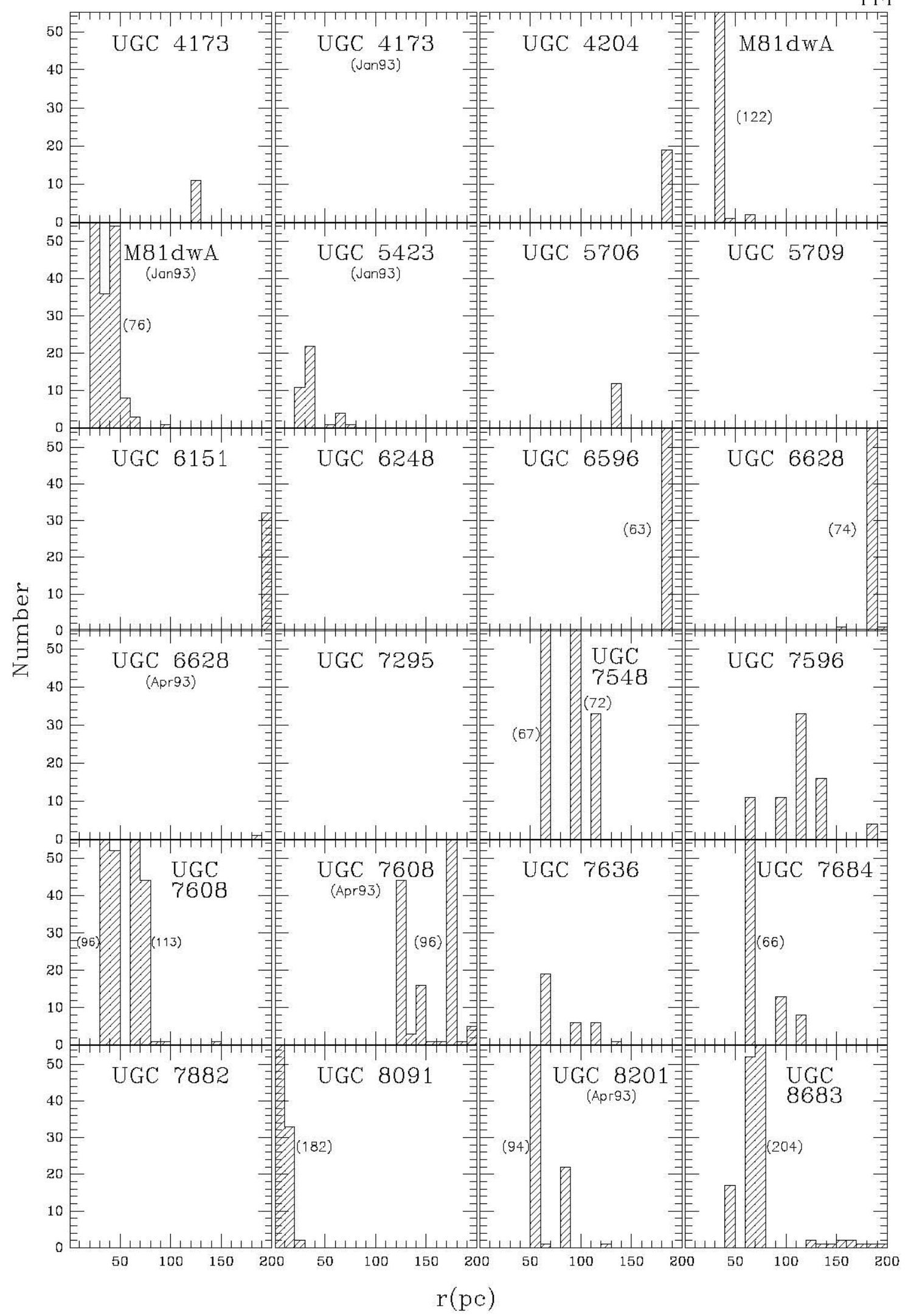

Figure 73: HII Region Size Distribution (cont.) 


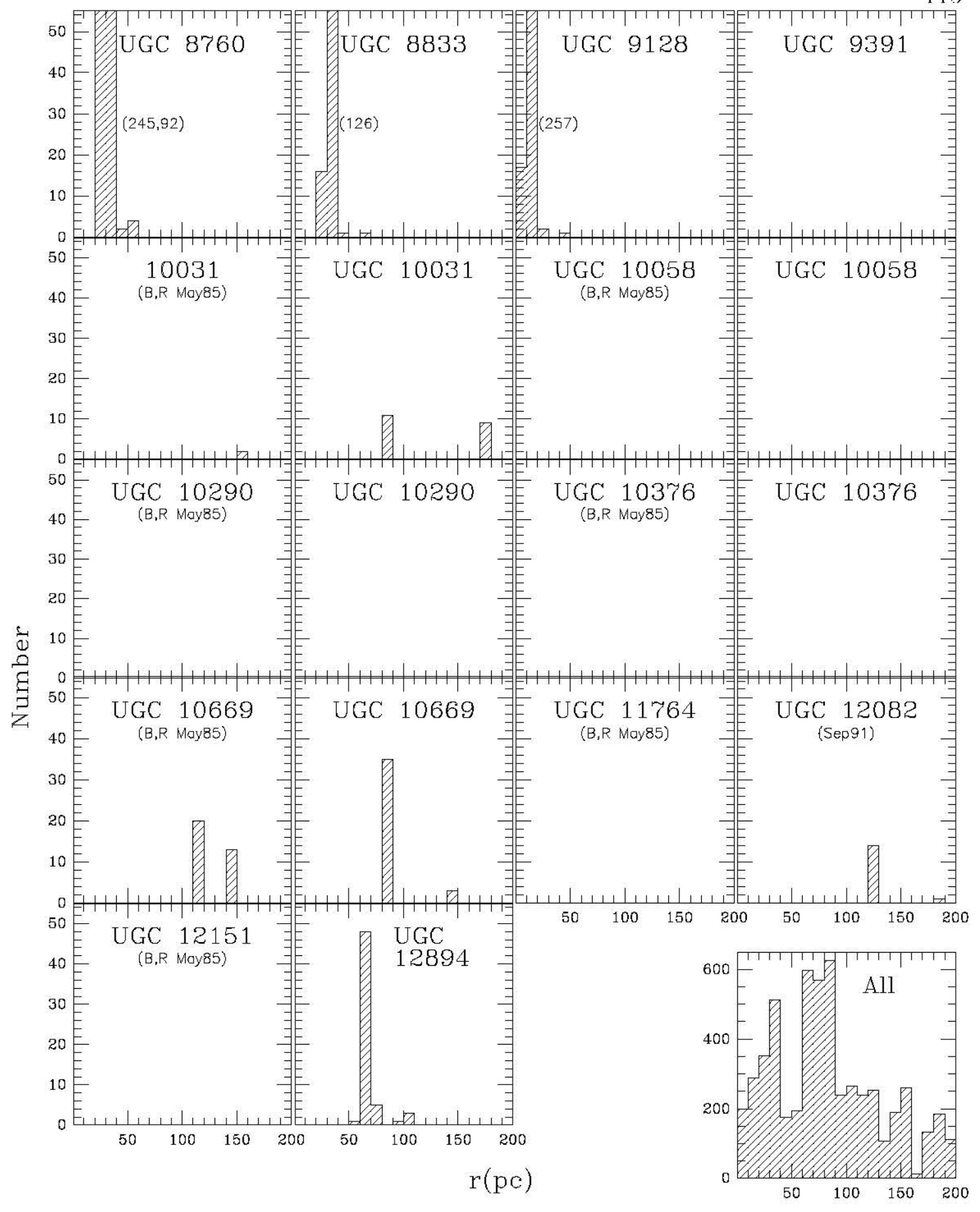

Figure 71: HII Region Size I)istribution (cont.) 


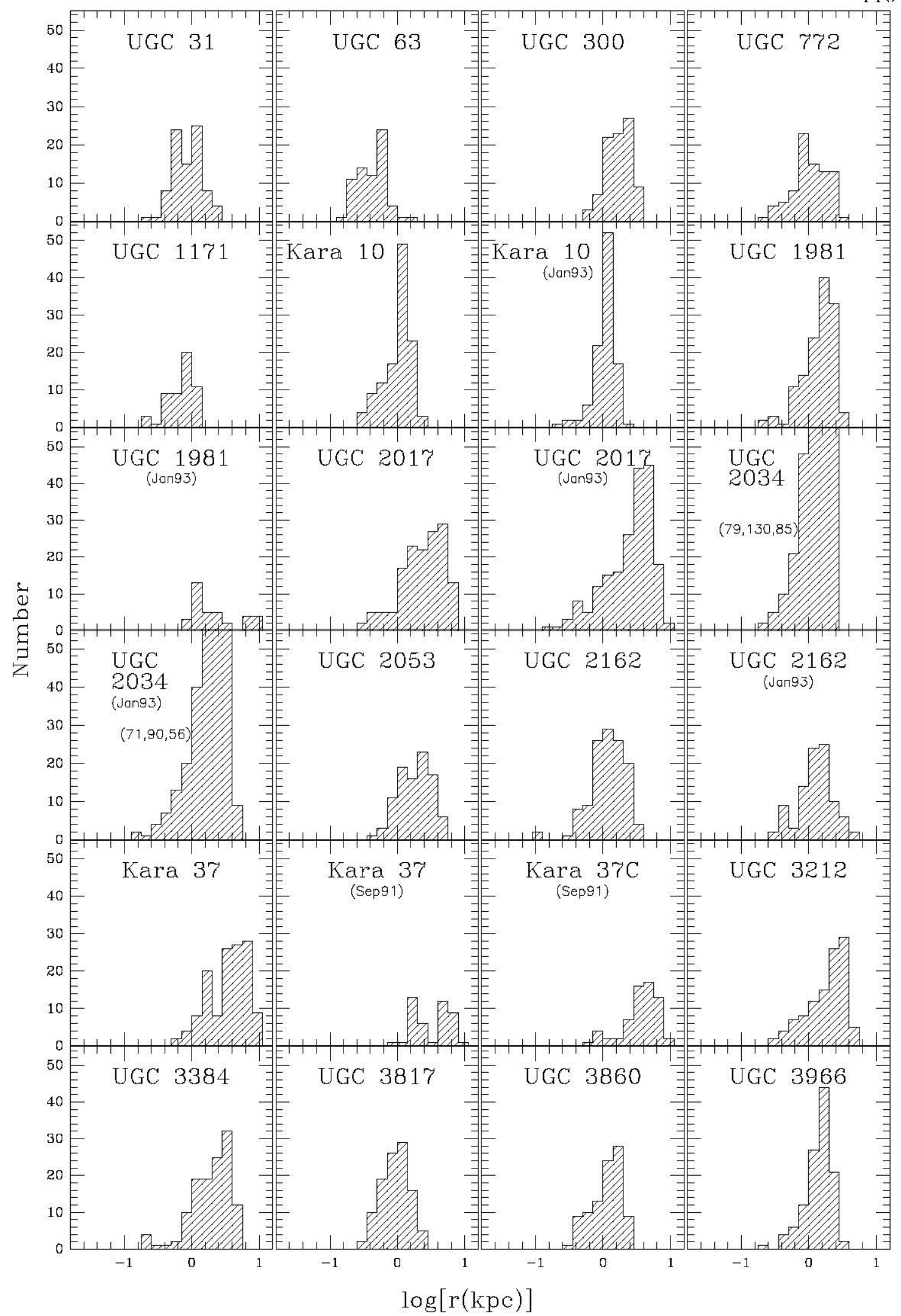

Figure 75: HII Region Radial Distribution 


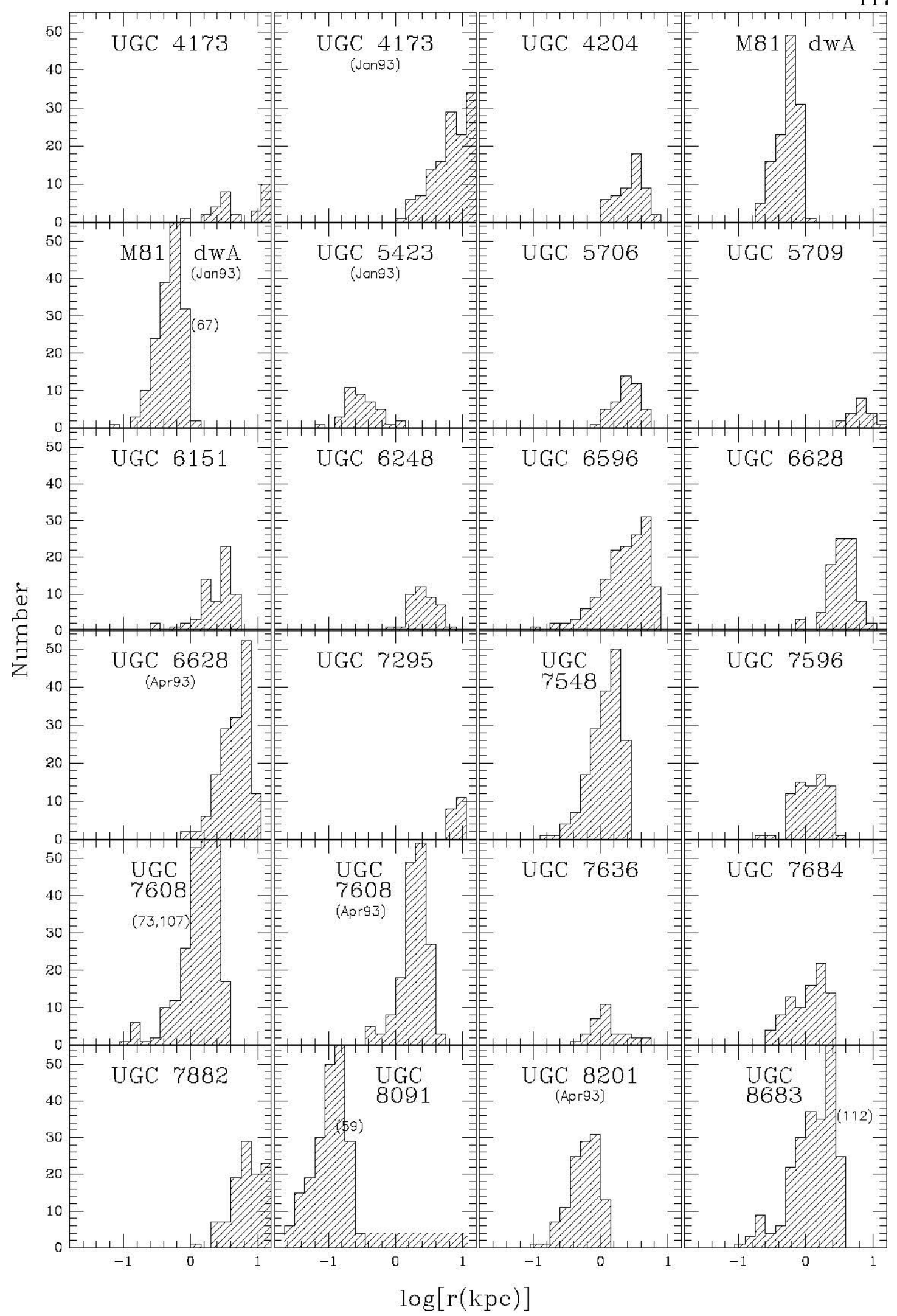

Figure 76: HII Region Radial l)istribution (cont.) 


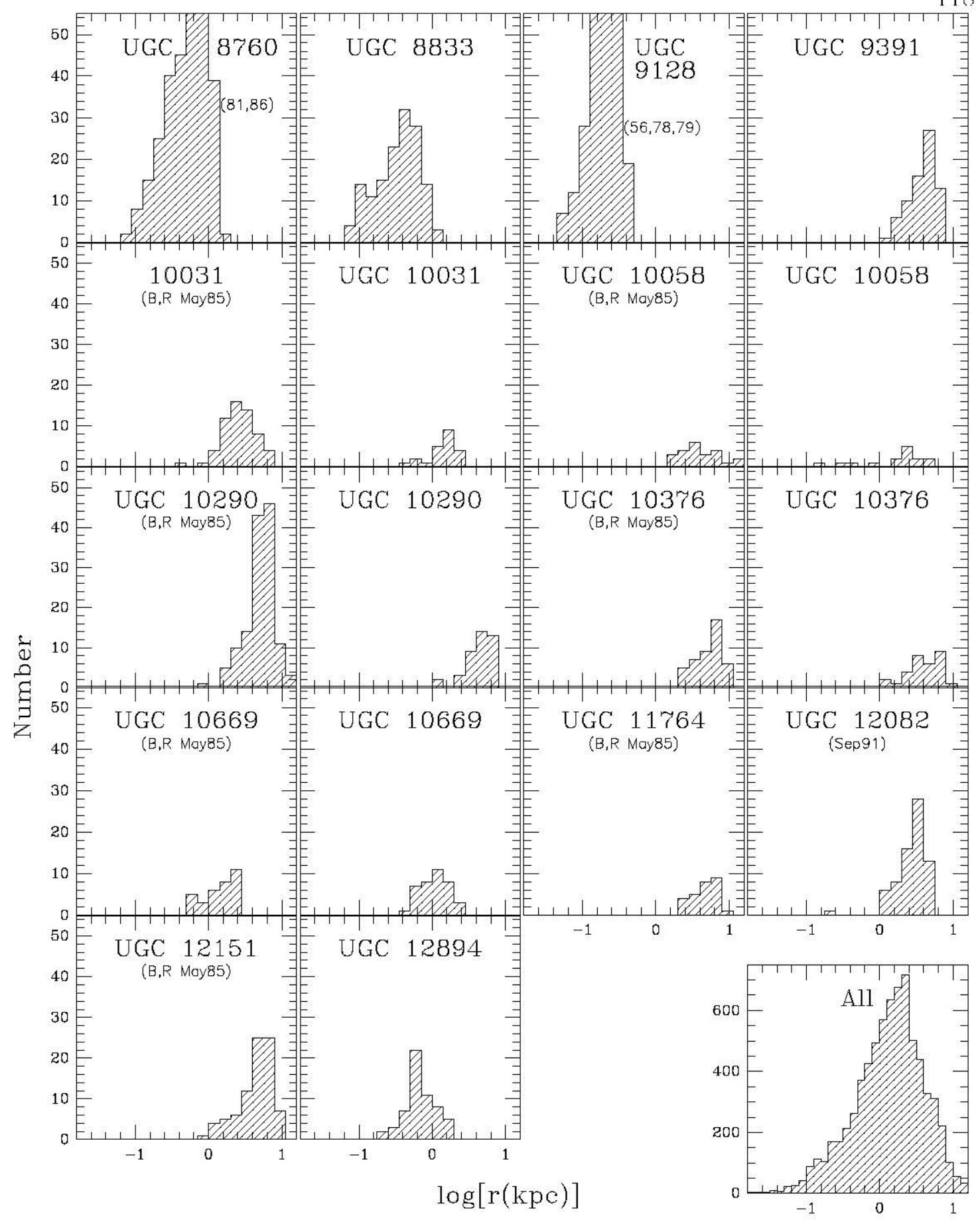

Figure 77: HII Region Radial Distribution (cont.) 


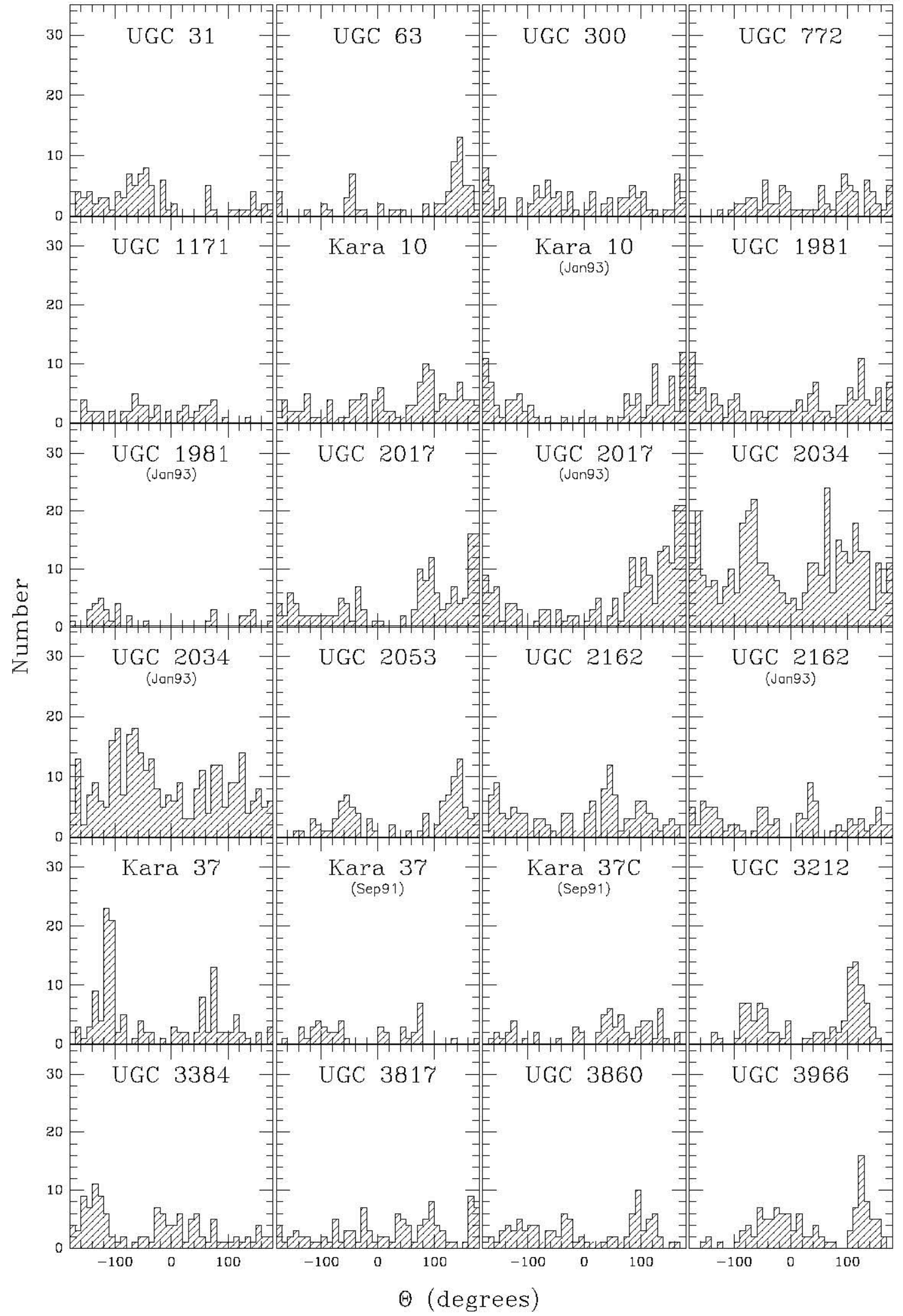

Figure 78: HII Region Azimuthal I)istribution 


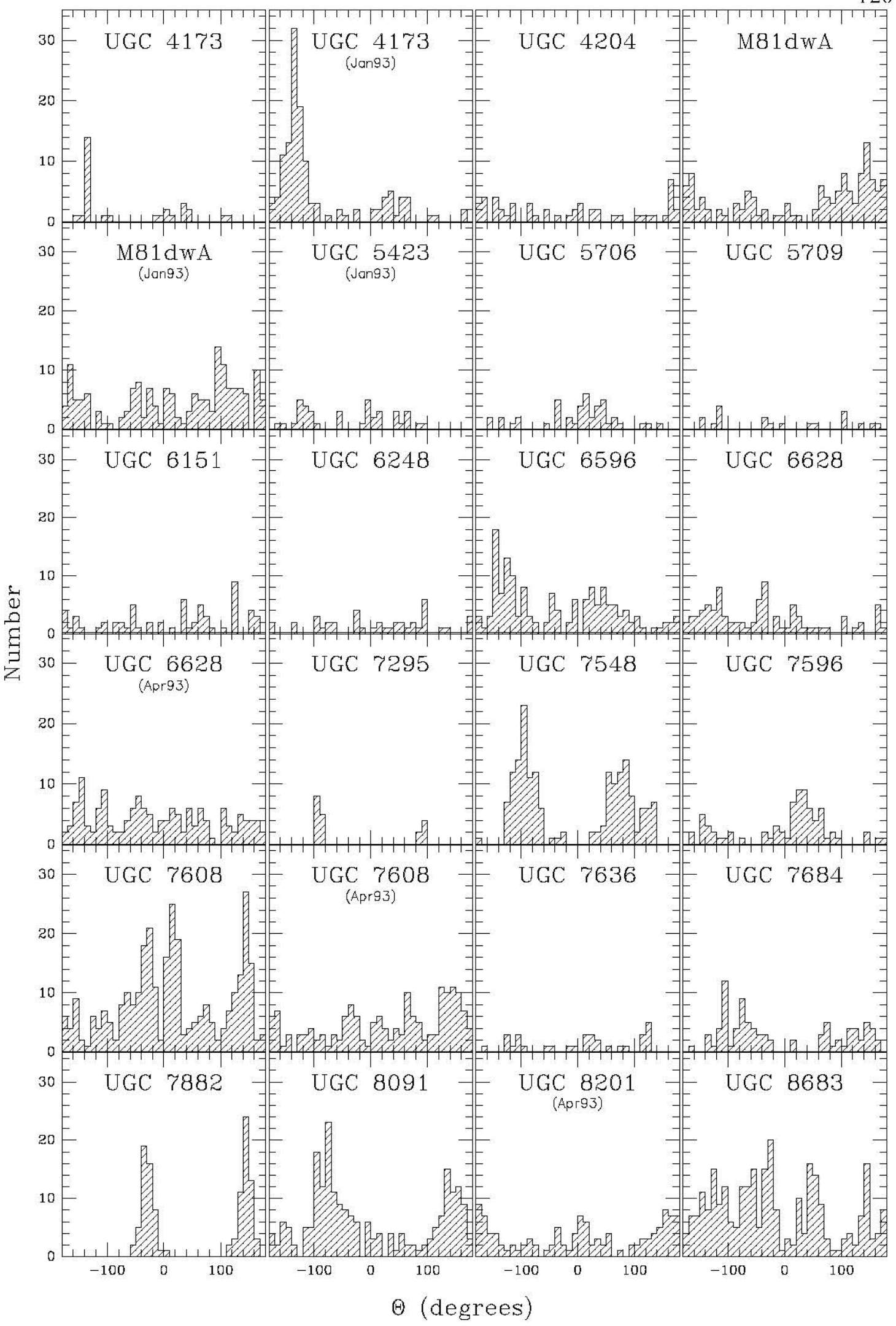

Figure 79: HIl Region Azimuthal I)istribution (cont.) 


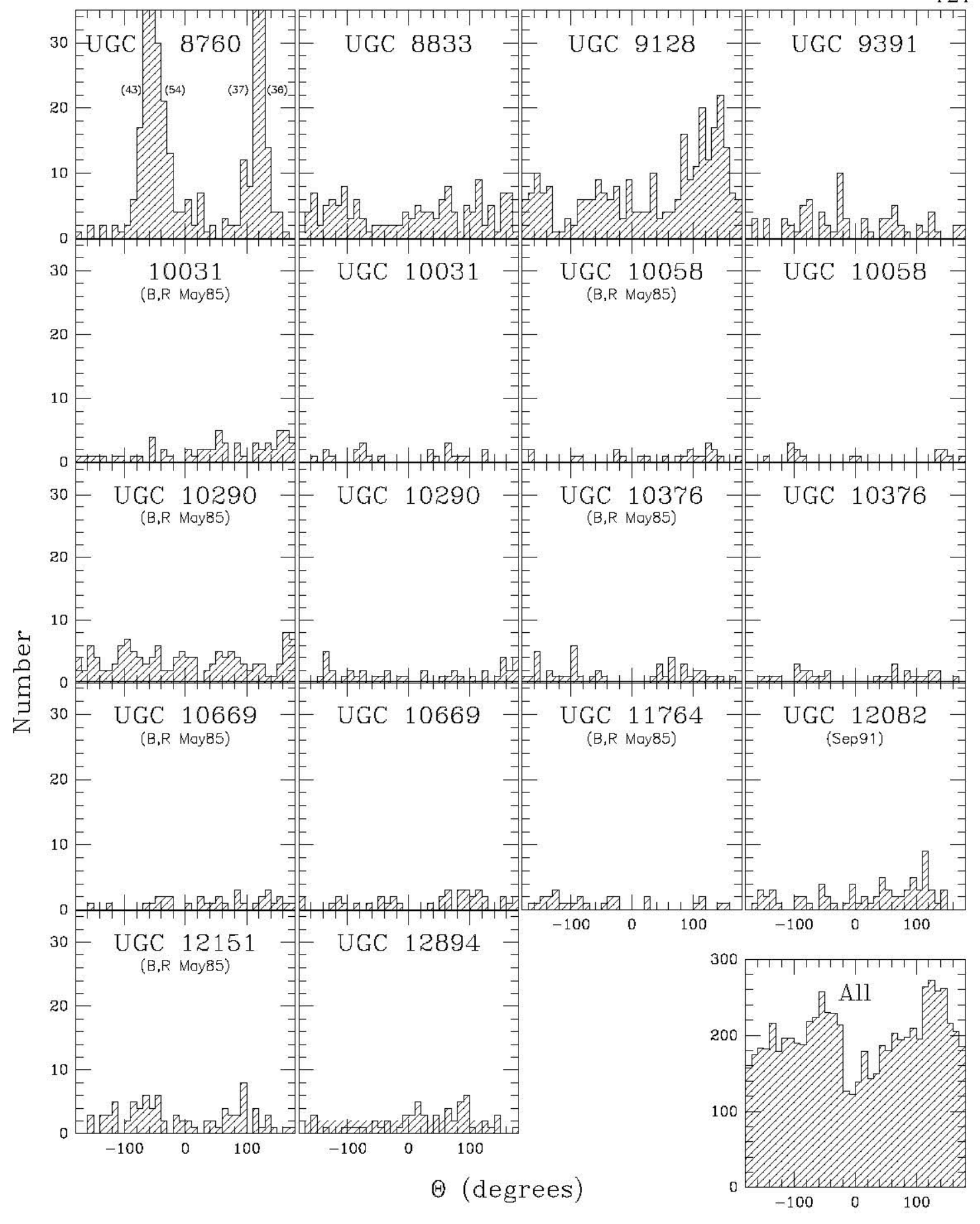

Figure 80: HII Region Azimuthal I)istribution (cont.) 


\section{Chapter 3}

\section{Corrections to Magnitude and HI Linewidth}

\subsection{Structural Parameters}

In order to derive physically meaningful information about the (3-dimensional) structure of the galaxies from the measured (2-dimensional) radial profile, it is necessary to adopt a model for the distribution of the luminous material within the galaxy. These models fall into two catcgories; a spheroidal bulge, or an exponential disk. There are three major parameterizations of the spheroidal bulge, the empirical Hubble law (Reynolds 1913, IIubble 1930), the theoretical models of King (1966), and the empirical de Vaucouleurs (1948) $r^{1 / 4}$ law. These three models fit elliptical galaxins almost equally well over a limited range of radii. It is neccssary to have surface brightness profiles extending over a large range of radii in order to differentiate between the models. 
The exponential disk model (de Vaucouleurs 1959) turns out to be a good fit to most disk like systems, such as the outer parts of spiral galaxies (Freeman 1970) and irregular galaxies (Impey, Bothun \& Malin 1988).

Ilowever, in the case of low surface brightness galaxies, the range of radii over which the profiles extend is often so limited that it is extremely difficult to distinguish between models. Note that the apparently dE galaxy UGC 31 (Figure 3) appears to be fairly well fit by an exponential disk, although a spheroidal model (such as an $r^{1 / 4}$ law) does provide a marginally better fit. In most other cases in the present sample, the profiles are much more irregular, making it difficult to decide, even on a case by case basis, which model provides a better fit.

I'herefore, it was decided to adopt the exponential disk model described below; both because previous work has confirmed the validity of this model in the low surface brightness and dI regime, and because no significant deviation from this model was found in the present work.

It should be noted, however, that a further complication arises because the disklike region may only lie in the outer region of the galaxy. This combination of bulge and disk components is well understood in larger disk galaxies, but the faintness of dwarfs makes the profile decomposition into bulge and disk components more difficult. While muclear regions of enhanced star formation are not similar to bulge components, they do contribute to pronounced deviations from exponentials in the surface brightness profiles in the region dominated by star formation (o.g. UGC 2017, Figure 11: LGC 8091, Figure 43). 


\subsubsection{Extinction and Orientation Corrections to Surface Brightness}

The observed surface brightness and magnitude must be corrected for extinction, both from dust in our own Cialaxy $\left(A_{g a l}\right)$, and from that in the galaxy being observed $\left(A_{\text {int }}\right)$. In addition, a line-of-sight term must be included to correct the surface brightness to a face-on orientation, since it depends on the area over which the luminous matter appears to be distributed. This is simply $A_{\text {los }}=2.5 \log \cos i$, depending only on the derived inclination $i$. 'The corrected surface brightness, $\mu^{C}$, is therefore given by the following equation:

$$
\mu^{o}=\mu^{o b s}-A_{g a l}-A_{\text {int }}-A_{\text {los }} .
$$

The $A_{\text {gal }}(B)$ term for the $\mathrm{LGC}$ galaxies is taken from the RC3 (de Vaucouleurs, et al. 1991), and is based on the HI observations of Burstein and Heiles (1984). For the remaining galaxies, the value was simply interpolated from that for nearby galaxies. A relationship between the extinction in $B, R$ and $I$,

$$
A(R)=0.62 A(B) ; \quad A(I)=0.11 A(B)
$$

was obtained from a tabulation of interstellar extinction as a function of wavelength (Scheffler \& Hilsässer 1988), and from l.11 et al. (1992).

I'he value of $A_{i n t}$ depends on the inclination, $i$, of the galaxy (assuming it is a disk, as discussed above). This is obtained from the following formula:

$$
\cos ^{2} i=\frac{(b / a)^{2}-q_{0}^{2}}{1-q_{0}^{2}}
$$

The value of $q_{0}$, the intrinsic axial ratio, is often taken to be 0.20 for spiral galaxies, based originally on the work of IIolmberg (1958) (Bottinelli el al. 1983). A recent 
study of a large sample of dwarf galaxies by Staveley-Smith, Davies \& Kinman (1992) lead them to determine the most likely value for $q_{0}$ given $b / a$. Using the relationship between $\phi(b / a)$, the observed axial ratio distribution function, and $\int\left(q_{0}\right)$, the intrinsic axial ratio distribution function for an oblate cllipsoidal figure (Sandage, frecman, \& Stokes, 1970; Mihalas \& Binney, 1981):

$$
p h i(b / a)=b / a \int_{0}^{b / a} \frac{\int\left(q_{0}\right) d q_{0}}{\sqrt{\left(1-q_{0}^{2}\right)\left(b / a-q_{0}^{2}\right)}}
$$

they lound

$$
q_{0}=0.65(b / a)-0.072(b / a)^{3.9}
$$

provided a good fit to the observational data. 'This tends to yicld values for qo which are greater than 0.20 , which is perhaps to be expected since the disk in these smaller systems are likely to be less flattened. As noted by McGaugh \& Bothun (1994), the actual value adopted for $q_{0}$ is not crucial since its effect on the value of $i$ is small compared to the uncertainty in $b / a$. We adopt the formulation of Staveley-Smith et al. (equation 3.5) for the intrinsic axial ratio for the remainder of this work.

I'he actual relation for $A_{\text {int }}(i)$ adopted is that of 'lully \& Fouqué (1985):

$$
A_{\text {in.t }}=-2.5 \log \left[\int\left(1+e^{-\tau \text { seci }}\right)+\left(1-2 \int\right)\left(\frac{1-e^{-\tau \text { seci }}}{\tau \text { scci }}\right)\right] \text {. }
$$

where $f$ is the fraction of light which is in front of the absorbing disk, and is taken to be 0.25 . The optical depth, $\tau$, is taken to be 0.55 in the $B$-band, while it is only 0.31 in the $R$-band and 0.21 in the $I$-band, from the relation between $A(B), A(R)$ and $A(I)$ given above. This correction is the one adopted for spirals by Pieree \&. Tully (1992) and Lu et al. (1992) among others.

The Tully \& Fouqué formulation for internal extinction is applicable to normal spiral galaxies. IIowever, it is questionable whether this, or any other "simple" 
extinction law is suitable for dwarf galaxies with their extremely low metallicities. and irregular distribution of gas and light. The value for $A_{\text {int. }}$ derived from equation (3.6) turns out to be large $(>0.4 \mathrm{mag})$ for most of the sample.

It was therefore decided not to include the $A_{i n t}$ term in the surlace brightness or magnitudes which are calculated. No correction for internal extinction was applicd by Carignan t $a l$. (1990) when calculating the optical characteristics for GR8, since they felt the value of the term was too uncertain. Staveley-Smith et al. (1992) also apply no correction for internal extinction, stating that in dwarl galaxies (which are interpreted as having fat, inflated disks) any such term is small. 'This agrees with the carly work of Holmberg (1958), who found no indication of an inclination dependent internal extinction in the 10 irregular galaxies in his sample.

The value of the corrections $A_{g a l}$ and $A_{\text {int }}$ (this correction is not applied, and only listed for relerence) for the $B$-band, as well as the line-of-sight correction term (for surlace brightlıess only), are listed in Table 5 for each galaxy.

\subsubsection{Exponential Disk Profiles}

If the underlying light distribution of the galaxy is in the form of an exponential disk of radius $r$,

$$
\Sigma(r)=\Sigma_{0} c^{-r / \alpha}
$$

then the surface brightness profile will be well fit by a straight line characterized by the following equation:

$$
\mu(r)=\mu_{0}+1.086 \mathrm{r} / \alpha
$$


where $\mu_{0}=-2.5 \log \Sigma_{0}$ is the central surface brightness of the disk and $\alpha$ is the scale length of the disk.

The total magnitude can be calculated for the galaxy by combining an isophotal flux (within a reasonably bright isophote) with an extrapolated flux from the outer exponential regions of the galaxy,

$$
\Sigma_{\text {tolat }}=\Sigma_{<R}+\Sigma_{>K}
$$

where $R$ is the eflective radius of the isophote lor which the isophotal flux $\left(\Sigma_{<R}\right)$ was measured. Clcarly,

$$
\Sigma_{<R}=10^{-0.4 m_{R}},
$$

where $m_{P}$ is the isophotal magnitude at radius $r=R$ and the remaining flux is

$$
\Sigma_{>K}=\int_{R}^{\infty} 2 \pi r 10^{-0.1 / t_{0}} e^{-r / \rho} d r
$$

This reduces to

$$
\Sigma_{>R}=2 \pi \alpha^{2} 10^{-0.1 \mu_{0}} c^{-R / \alpha}(R / \alpha+1)
$$

If we adopt the notation used by Lu $\mathrm{el}$ al. (1993) (see also Lu 1993) and define $\phi(x)=(1+x) c^{-x}$, and $m_{\infty}=-2.5 \log 2 \pi \alpha^{2} 10^{-0.1 \mu_{0}}$ (the total magnitude of a galaxy which is a purcly exponential disk), the total magnitude $m_{T}$ is then given by

$$
m_{Y}=m_{R}-2.5 \log \left[1+10^{-\left(\frac{m_{\infty}-n_{R}}{2.5}\right)} \Phi(R / \alpha)\right]
$$

This value for the total magnitude must then be corrected for galactic extinction (yiclding a $m_{T}^{0}$ ), in the same manner as the surface brightness in the previous section. However, in the case of magnitudes, no correction for the line of sight is necessary (the $A_{l o s}$ term), and once again in this case, no correction for internal extinction is applied. 
When the outermost contour level in the surface brightness profile is brighter than the standard isophotal level $\left(e . g . B_{25}\right)$ it was necessary to obtain the isophotal magnitude by extrapolation. This is done as above:

$$
m_{25}=m_{R}-2.5 \log \left\{1+10^{-\left(\frac{m_{x_{2}}-m_{R}}{25}\right)}\left[\Phi(R / \alpha)-\Phi\left(r_{25} / \alpha\right]\right\}\right. \text {. }
$$

where $r_{25}$ is the effective radius at a corrected surface brightness level, $\mu^{C}$, of $25 \mathrm{mag} \operatorname{arcsec}^{-2}$.

The uncorrected disk parameters and magnitudes are listed for each galaxy in Table 5. The inclination and position angle were obtained from the outer isophotes of the $I$-band image (or $R$ or $B$ if no $I$ was available). The uncorrected central surface brightness $\left(\mu_{B}^{0}, \mu_{T}^{0}\right)$ and scale length $\left(\alpha_{B}, \alpha_{I}\right)$ are listed, followed by the total magnitude in $B$ and I that would result from a purely exponential disk with the given $\alpha$ and $\mu^{0}$. Finally, the galactic and internal (not applied) extinction in the $B$-band and the line-of-sight correction for the surface brightness are listed.

The results of the exponential fitting, corrected as described above, are listed in Table 6 . The $B$ and $I$ central surlace brightness $\left(\mu_{B}^{(0, c)}, \mu_{T}^{(0, c)}\right)$ are listed as well as the effective radius at which the corrected surface brightness is equal to $25 \mathrm{mag}^{\mathrm{arcsec}}{ }^{-2}$ $\left(r_{25}^{B}\right)$ along with the Holmberg radius $\left(r_{26.5}^{B}\right)$. Next the aperture magnitudes at the 25th and 26.5 isophote for $B$ and 22.5 and 24.5 for $I$ are given. Finally the total corrected magnitude with $\left(B_{T}^{0}, I_{T}^{0}\right)$ and without $\left(B_{T}^{0-}, I_{T}^{0-}\right)$ the identified IIII regions and stars within the galaxy are listed.

For the galaxics with previous (CI) photometry (Figure 2), we can compare their total corrected magnitudes with ours. For UGC:8091 (GR8) Carignan et al. (1990) list $B_{T}^{0}=14.63$, while we find 14.53 (with some cirrus present). This would appear to be satisfactory, especially since the brightest star "in" GR8 is a foreground 
Table 5: Uncorrected Disk Parameters and Magnitudes

\begin{tabular}{|c|c|c|c|c|c|c|c|c|c|c|c|}
\hline Galaxy & $\begin{array}{l}\mathrm{i} \\
\left({ }^{\circ}\right)\end{array}$ & $\begin{array}{l}\text { PA } \\
\left({ }^{\circ}\right)\end{array}$ & $\begin{array}{r}\mu_{B}^{0} \\
\left(\frac{\operatorname{madg}}{\operatorname{arcsec}{ }^{2}}\right)\end{array}$ & $\begin{array}{l}\alpha_{B} \\
(")\end{array}$ & $\begin{array}{r}\mu_{I}^{0} \\
\left(\frac{\mathrm{uragg}_{\mathrm{a}}}{\operatorname{arcsec}}\right)\end{array}$ & $\begin{array}{l}\alpha_{I} \\
(")\end{array}$ & $\begin{array}{r}B_{\infty} \\
(\mathrm{mag})\end{array}$ & $\begin{array}{r}I_{\infty} \\
(\mathrm{mag})\end{array}$ & $\begin{array}{c}\mathrm{A}_{\text {gal }}^{B} \\
\text { (mag) }\end{array}$ & $\begin{array}{r}A_{i n b l}^{B} \\
(\mathrm{mag})\end{array}$ & $\begin{array}{r}A_{\operatorname{los}} \\
\left(\frac{\operatorname{mecg}}{\operatorname{arcsec}}\right)\end{array}$ \\
\hline 31 & 50 & 52 & 21.42 & 7.6 & 20.23 & 9.0 & 15.31 & 13.78 & 0.08 & 0.40 & -0.48 \\
\hline 63 & 60 & 61 & 22.03 & 8.5 & 17.71 & 2.0 & 15.72 & 14.65 & 0.22 & 0.48 & -0.74 \\
\hline 300 & 39 & 43 & 23.66 & 16.4 & 21.86 & 7.1 & 15.75 & 15.78 & 0.04 & 0.34 & -0.27 \\
\hline 772 & 59 & 39 & 20.62 & 8.2 & $\ldots$ & $\ldots$ & 14.56 & $\ldots$ & 0.04 & 0.47 & -0.71 \\
\hline $\mid 171$ & 58 & 163 & 20.75 & 8.3 & $\ldots$ & . & 14.51 & . & 0.16 & 0.17 & -0.70 \\
\hline Klo & 57 & 129 & 21.10 & 18.5 & 23.82 & 21.1 & 16.11 & 15.33 & 0.13 & 0.16 & -0.6 .5 \\
\hline$K 10^{2}$ & 65 & 122 & 24.96 & 52.2 & 24.77 & 65.6 & 14.99 & 14.37 & 0.13 & 0.56 & -0.95 \\
\hline 1981 & 40 & 173 & 21.34 & 5.7 & 21.24 & 9.0 & 15.79 & 14.70 & 0.00 & 0.34 & -0.29 \\
\hline $1981^{2}$ & 54 & 2 & 23.35 & 16.6 & 21.86 & 10.9 & 15.70 & 15.12 & 0.00 & 0.43 & -0.58 \\
\hline 2017 & 61 & 40 & 23.93 & 15.3 & 22.60 & 13.5 & 16.31 & 15.44 & 0.31 & 0.51 & -0.80 \\
\hline $2017^{2}$ & 51 & 7 & 24.12 & 20.7 & 23.33 & 19.3 & 15.61 & 15.35 & 0.31 & 0.10 & -0.30 \\
\hline 2034 & 59 & 94 & 24.02 & 22.1 & $21.1 \overrightarrow{3}$ & 10.2 & 15.62 & 11.56 & 0.20 & 0.18 & -0.71 \\
\hline $2034^{2}$ & 31 & 124 & 22.93 & $25 . \overline{0}$ & 21.13 & 15.3 & 13.83 & 13.24 & 0.20 & 0.31 & -0.16 \\
\hline 2053 & 73 & 38 & 22.61 & 11.8 & 21.80 & 15.1 & 15.95 & 14.83 & 0.39 & 0.73 & -1.34 \\
\hline 2162 & 54 & 142 & 24.09 & 14.2 & 23.34 & 14.6 & 16.71 & 15.93 & 0.06 & 0.43 & -0.58 \\
\hline $2162^{2}$ & 76 & 116 & 23.93 & 14.3 & 24.50 & 32.7 & 17.37 & 16.17 & 0.06 & 0.80 & -1.52 \\
\hline $\mathrm{K} 37$ & 72 & 160 & 25.20 & 30.0 & 21.30 & 11.7 & 13.83 & 13.85 & 3.00 & 0.69 & -1.25 \\
\hline $\mathrm{K} 37^{2}$ & 65 & 137 & 25.64 & 18.0 & 23.77 & 20.3 & 12.78 & 11.16 & 3.00 & 0.36 & -0.95 \\
\hline $\mathrm{K} 37 \mathrm{C}^{2}$ & 25 & 76 & 25.56 & 119.1 & 22.54 & 37.1 & 10.27 & 11.46 & 3.00 & 0.30 & -0.11 \\
\hline 3212 & 71 & 23 & 22.58 & 6.2 & 21.51 & 5.8 & 17.08 & 16.46 & 0.53 & 0.68 & -1.23 \\
\hline 3384 & 40 & 83 & 22.63 & 5.1 & 21.13 & 5.6 & 16.76 & 15.34 & 0.57 & 0.34 & -0.28 \\
\hline 3817 & 51 & 140 & 23.33 & 9.4 & 22.12 & 9.6 & 16.49 & 15.43 & 0.36 & 0.41 & -0.51 \\
\hline 3860 & 17 & 15 & 19.18 & 11.7 & $\ldots$ & $\ldots$ & 12.23 & $\ldots$ & 0.23 & 0.38 & -0.12 \\
\hline 3966 & 17 & 28 & 23.80 & 19.5) & 23.36 & 23.8 & 15.15 & 11.70 & 0.22 & 0.38 & -0.11 \\
\hline 4173 & 71 & 137 & 24.45 & 9.6 & 22.50 & 11.3 & 18.44 & 16.19 & 0.10 & 0.68 & -1.24 \\
\hline $4173^{2}$ & 62 & 131 & 23.45 & 19.0 & 22.69 & 18.9 & 15.59 & 14.89 & 0.10 & 0.51 & -0.81 \\
\hline 4204 & 50 & 102 & 23.30 & 9.5 & 21.74 & 8.9 & 16.66 & 15.30 & 0.13 & 0.40 & -0.48 \\
\hline M81d & 39 & 9 & 24.20 & 20.9 & 23.57 & 57.5 & 15.72 & 12.94 & 0.09 & 0.34 & $-0.2 \bar{\tau}$ \\
\hline MI $1 \mathrm{dw} \mathrm{A}^{2}$ & 59 & 82 & 23.68 & 12.3 & 21.72 & 38.7 & $\mid 6.71$ & 15.30 & 0.09 & 0.18 & -0.72 \\
\hline $5423^{2}$ & 59 & 138 & 22.07 & 10.0 & 20.80 & 9.6 & 15.12 & 11.31 & 0.20 & 0.17 & -0.71 \\
\hline 5706 & 61 & 103 & 24.62 & 27.6 & 23.58 & 27.5 & 16.02 & 14.99 & 0.02 & 0.51 & -0.80 \\
\hline 5709 & 61 & 117 & 22.58 & 11.0 & 21.30 & 11.4 & 15.95 & 14.61 & 0.04 & 0.50 & -0.79 \\
\hline 6151 & 18 & 175 & 23.46 & $20 . \bar{t}$ & 22.60 & 23.2 & 14.92 & 13.82 & 0.00 & 0.28 & -0.0 .5 \\
\hline 6248 & 36 & 28 & 24.14 & 20.4 & 22.81 & 22.6 & 15.73 & 14.20 & 0.03 & 0.33 & -0.22 \\
\hline 6596 & 62 & 123 & 22.87 & $10 . \overline{7}$ & 22.8 .5 & 20.8 & 16.36 & 14.89 & 0.00 & 0.51 & -0.82 \\
\hline 6628 & 11 & 60 & 22.15 & 30.7 & 21.17 & 33.8 & 13.21 & 11.76 & 0.01 & $0.3 \overrightarrow{3}$ & -0.31 \\
\hline $6628^{2}$ & 34 & 18 & 22.20 & 35.6 & $\ldots$ & $\ldots$ & 12.60 & $\ldots$ & 0.01 & 0.32 & -0.21 \\
\hline 7295 & 74 & 1 & 22.50 & 5. & 19.67 & 3.7 & 17.97 & 15.94 & 0.00 & 0.74 & -1.37 \\
\hline 7548 & 69 & 174 & 22.38 & $10 . \overline{0}$ & 21.60 & 11.4 & 16.02 & 15.13 & 0.13 & 0.63 & -1.10 \\
\hline 7596 & 69 & 134 & 22.05 & 9. & 21.13 & 12.7 & 16.08 & 14.51 & 0.00 & 0.63 & -1.12 \\
\hline 7608 & 53 & 64 & 24.26 & 15.6 & 23.98 & 37.9 & 16.72 & 14.51 & 0.00 & 0.42 & -0.5 .5 \\
\hline $7608^{2}$ & 53 & 38 & 23.71 & 19.7 & & & 13.38 & & 0.00 & 0.12 & $-0 . \overline{s i s}$ \\
\hline 7636 & 51 & 1 & 24.07 & 31.8 & 22.62 & 38.9 & 11.91 & 13.05 & 0.00 & 0.10 & -0.30 \\
\hline 7684 & 58 & 42 & 21.80 & 8. & 20.37 & 10.9 & 15.71 & 1.3 .69 & 0.07 & 0.46 & -0.67 \\
\hline 7882 & 65 & 59 & 22.26 & 6.4 & 21.44 & 8.3 & 17.82 & 16.45 & 0.01 & 0.56 & -0.94 \\
\hline 8091 & 52 & 44 & 23.30 & 15.8 & 22.47 & 20.6 & 15.67 & 14.28 & 0.04 & 0.41 & -0.52 \\
\hline $8201^{2}$ & 46 & 88 & 22.48 & 37.5 & $\ldots$ & $\ldots$ & 12.87 & $\ldots$ & 0.04 & 0.37 & -0.39 \\
\hline 8683 & 59 & 111 & 22.71 & 11.8 & 23.39 & 30.9 & 15.91 & 14.50 & 0.00 & 0.18 & -0.72 \\
\hline 8760 & 79 & 33 & 22.15 & 15.1 & 22.10 & 20.5 & 16.05 & 15.08 & 0.00 & 0.92 & -1.81 \\
\hline 8833 & 48 & 150 & 22.20 & 10.3 & 22.31 & 17.3 & 15.17 & 11.15 & 0.00 & 0.39 & -0.11 \\
\hline 9128 & 61 & 41 & 21.78 & 11.7 & 21.21 & 12.2 & 15.06 & 14.39 & 0.00 & 0.50 & -0.79 \\
\hline 9391 & 50 & 52 & 22.37 & 14.0 & 21.42 & 16.8 & 15.01 & 13.66 & 0.00 & 0.40 & -0.48 \\
\hline 10031 & $4 \pi$ & $1 \bar{i}$ & 23.67 & 12.0 & 23.53 & 21.4 & 16.57 & 15.19 & 0.01 & 0.38 & -0.41 \\
\hline 10058 & 35 & 20 & 23.29 & 10.9 & 23.38 & 20.1 & 16.08 & 14.95 & 0.18 & 0.32 & -0.21 \\
\hline 10290 & 13 & 177 & 22.95 & 16.1 & 22.28 & 39.7 & 11.77 & 12.38 & 0.36 & 0.36 & -0.33 \\
\hline 10376 & 39 & 22 & 23.13 & 10.2 & 22.17 & 8.3 & 16.57 & 15.75 & 0.01 & 0.31 & -0.27 \\
\hline 10669 & 47 & 55 & 23.73 & 12.3 & 23.04 & 12.8 & 16.46 & 15.76 & 0.15 & 0.38 & -0.42 \\
\hline 11764 & 27 & 12 & 23.71 & 12.0 & $\ldots$ & $\ldots$ & 16.24 & $\ldots$ & 0.16 & 0.30 & -0.12 \\
\hline $12082^{2}$ & 54 & 175 & 24.27 & 28.0 & 22.95 & 31.4 & 15.15 & 3.77 & 0.33 & 0.43 & -0.59 \\
\hline 12151 & 51 & 179 & 23.37 & 23.0 & $\ldots$ & $\ldots$ & 14.74 & $\cdots$ & 0.21 & 0.41 & -0.50 \\
\hline 12894 & 46 & 148 & 24.55 & 88.2 & 23.53 & 60.4 & 12.66 & 12.73 & 0.47 & 0.37 & -0.40 \\
\hline
\end{tabular}


Table 6: Corrected Magnitudes

\begin{tabular}{|c|c|c|c|c|c|c|c|c|c|c|}
\hline$y$ & $\begin{array}{l}\mu_{B}^{(0, c)} \\
\quad\left(\frac{m}{\operatorname{arc} s}\right)\end{array}$ & $\begin{array}{l}(0, c) \\
\left(\frac{0, c}{2}\right) \\
(2)\end{array}$ & $\begin{array}{l}r_{2.3}^{B} \\
\left({ }^{\prime \prime}\right)\end{array}$ & $\begin{array}{r}r_{26, \overline{3}}^{B} \\
\text { (") }\end{array}$ & $\begin{array}{c}B_{25}^{c} / B_{26.5}^{c} \\
(\mathrm{mag})\end{array}$ & $\begin{array}{c}I_{22.5}^{c} / I_{24.5}^{c} \\
(\mathrm{mag})\end{array}$ & $\begin{array}{c}B_{T}^{0} \\
(\mathrm{~m}\end{array}$ & $B_{T}^{0-}$ & & $I_{T}^{0-}$ \\
\hline 31 & 21.82 & 20.67 & 25.6 & 37.5 & $14.62 / 14.56$ & $13.59 / 13.37$ & 14.54 & 14.97 & 13.29 & 13.64 \\
\hline 63 & 22.54 & 18.35 & 23.6 & 34.8 & $14.87 / \ldots$ & $/ 15.30$ & 14.76 & 5.12 & 5.28 & 3.96 \\
\hline 300 & 23.90 & 22.11 & 24.0 & 41.3 & $5.90 / \ldots$ &.$/ 15.71$ & 15.37 & 15.61 & 5.46 & 15.82 \\
\hline 772 & 21.29 & $\ldots$ & & 87.1 & 1 & $\ldots / \ldots$ & 13.69 & 14.14 & $\cdots$ & \\
\hline & 21.28 & - & 34.2 & 51.0 & $.40 /$. &.$/ .$. & 13.37 & 13.75 & & \\
\hline & 21.62 & 21.12 & $\ldots$ & 11.6 & $\ldots / 15.65$ &.$/ 15$. & 1.3 .11 & 13.66 & 14.85 & 15.06 \\
\hline & 5.78 & 25.66 & . . & 55.1 & $\ldots / 15.13$ & $\ldots / \ldots$ & 14.51 & 14.60 & 4.14 & 1.22 \\
\hline & 21.63 & 21.53 & 20.4 & 27.7 & $16.25 / 16.13$ & $5.45 / 14.75$ & 16.07 & 16.60 & 4.55 & 4.99 \\
\hline & 3.93 & 22.43 & 24.1 & 55.0 & $15.67 / \ldots$ & $\ldots / 15.02$ & 15.23 & 15.29 & 4.76 & 4.8 .5 \\
\hline & 4.41 & 23.26 & 17.9 & 38.6 & $15.52 / 15$. &.$/ 14.95$ & 15.18 & & 4.64 & 4.92 \\
\hline & 1.31 & 23.89 & 22.6 & 55.2 & $15.36 / \ldots$ & $.11 !$ & & & & 5.00 \\
\hline & 24.54 & 21.78 & 34.8 & 60.2 & $1.1 .21 / 1.1 .1$ & $3.78 / 13.63$ & 1.08 & $\mid 1.81$ & 3.57 & 1.30 \\
\hline & 22.90 & 21.20 & 5.8 & 76.3 & $13.85 / \ldots$ & $.86 / 13.25$ & 13.45 & 13.72 & 3.09 & $3.4 \overline{5}$ \\
\hline & 3.56 & 22.97 & 27.0 & 42.8 & $14.09 / 14.1$ & $\ldots / 13$ & & & & 13.99 \\
\hline & 4.61 & 23.89 & 14.0 & 33.6 & $16.65 / 16$ & .116 & & & 5.61 & 15.87 \\
\hline & 25.39 & 2.5 .99 & $\ldots$ & 26.4 & $\ldots / 15.81$ & $\ldots /$. & 15.72 & & 5.50 & 15.70 \\
\hline & 23.15 & 21.13 & $\ldots$ & 110.8 & $\ldots / \ldots$ & $.36 / 11.26$ & 9.81 & & 11.22 & 11.81 \\
\hline & 23.58 & 23.39 & $\ldots$ & 11.9 &..$/ \ldots$ & $2.12 / 12.30$ & 9.71 & 78 & 12.11 & $|2 . \overline{\mid}|$ \\
\hline & 2.67 & 21.33 & $\ldots$ & 308.3 & $\ldots / \ldots$ & $0.48 / \ldots$ & 7.25 & 29 & 9.81 & 10.08 \\
\hline & 28 & 22.50 & 5.0 & 3.8 & $.06 / 15.0$ & $\ldots / 14.89$ & 14.99 & & 14.82 & 15.67 \\
\hline & 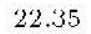 & 21.16 & & .4 & & $\ldots / 1$ & & & & 15.15 \\
\hline & 2 & $22.4 \pi$ & 19.4 & 3 & 715. & .1 & & & 4.48 & 5.20 \\
\hline & 1.6 & $\ldots$ & $\ldots$ & 6 &..$/ \ldots$ & $\ldots / \ldots$ & & & . & \\
\hline & 1.00 & 23.68 & 30.7 & 17.1 & $5.01 / 11.8$ & $\ldots / 11$ & & & 1.01 & 11.52 \\
\hline & 2 & 23.69 & & 24.0 & $\ldots / 1$ & $\ldots / 1$ & & & 5.01 & 5.36 \\
\hline & .1 & 23.45 & 30.2 & 68.8 & $14.77 / \ldots$ & $\ldots / 1$ & 54 & & 4.08 & 4.28 \\
\hline & & 22.16 & 16.7 & 30.3 & & $\ldots / 1$ & & & 4.73 & 5.13 \\
\hline & & 23.80 & 23.0 & 45.4 & & $\ldots / 1$ & & & 2.89 & 2.94 \\
\hline & 21.31 & 2.5 .40 & $\cdots$ & 30.2 & $\ldots / 1$ & $\ldots /$ & 91 & & 02 & 15.30 \\
\hline 512 & 22.57 & 21.12 & 27.8 & 11.3 & $42 / 1$ & $.95 / 13$ & & & & 13.83 \\
\hline & $A_{1}$ & 24.37 & 10.6 & 42.4 & & $\ldots / 1$ & & & & $b b$ \\
\hline & .33 & 22.07 & 23.4 & 39.7 & $15.14 /$ & $4.17 / 1$ & & & 3.77 & 13.83 \\
\hline & - & 22.65 & 37.2 & 63.2 & & $5.05 / 1$ & & & 3.59 & 13.75 \\
\hline & & 23.03 & 9.6 & 52.1 & 1 &..$/ 1$ & & & 98 & 14.06 \\
\hline & & & 22.4 & .2 & & 11 & & & & 14.43 \\
\hline & & 18 & .3 & .8 & & $2.87 / 11$ & & & 11.75 & 11.81 \\
\hline & 2.10 & & .3 & .1 & $\ldots$ & $\ldots /$. & 31 & & & \\
\hline & & 04 & 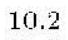 & & & $\ldots / 1$ & & & 71 & .77 \\
\hline & & 4 & 24 & & & $\ldots / 1$ & & & 02 & 48 \\
\hline & & 5 & 22 & & & $68 / 1$ & & & 39 & 62 \\
\hline & & 4.53 & 12.7 & 8 & .... &..$/ 15$ & & & 4.40 & 4.4 \\
\hline & .26 & & 78.9 & $111 . .5$ & $/ 12.7$ &.$\ldots$ & 66 & & & \\
\hline & .56 & 3.11 & 28.1 & .5 & 1. & $19 \%$ & 13 & & 2.83 & 12.8 .5 \\
\hline & 1 & 21.02 & 23.6 & 0 & & $28 / \ldots$ & & & 2.99 & 3.12 \\
\hline & & 22.37 & & 4 & 17.6 & $3.50 / 13$ & & & 13.44 & 14.33 \\
\hline & 23.78 & 22.97 & 31.4 & 44.9 & $14.68 / \ldots$ & $\ldots / 13.9$ & 14.53 & 15.43 & 13.64 & 14.21 \\
\hline 820. & 22.83 & $\ldots$ & 90.3 & 157.3 & $12.00 / \ldots$ & $\ldots / \ldots$ & 11.87 & & - & \\
\hline & 13 & 21.11 & 23.7 & 12.9 & & $\ldots$ & 1.1 .83 & 15.34 & 3.53 & 13.9 .5 \\
\hline & & 23.91 & 8 & & &..$/ 1$ & & & 3.10 & 13.61 \\
\hline & & 22.75 & 26.8 & .3 & & $.28 / 1$ & & & 3.86 & 11.20 \\
\hline & & & & & & & & & 3.80 & 14.44 \\
\hline & & & 0 & & & & & & 3.16 & 1.3 .33 \\
\hline & & & 17.5 & & & $.56 / \ldots$ & & & 4.78 & 13.59 \\
\hline & & & & & 17 & $.40 \%$ & & & 4.54 & 3.37 \\
\hline & & & 1 & & & $\ldots / \ldots$ & & & 2.066 & 11.0 \\
\hline & & 22.12 & & & & 11 & & & 5.15 & 13.82 \\
\hline & & 23.40 & & & & $5.96 / 15$ & & & 5.31 & 3.99 \\
\hline & & $\cdots$ & 10. & 0.2 & & $\ldots / \ldots$ & 15.66 & 16.03 & $\cdots$ & \\
\hline & & 23.39 & & 5.1 & & $.10 / 1$ & & & 2.65 & 13.21 \\
\hline & & .. & 40.6 & 70.2 & $14.17 / 13.99$ & $\ldots / \ldots$ & & & & \\
\hline 12894 & 24.49 & 23.73 & $\ldots$ & 170.0 & $\ldots / \ldots$ & $\ldots / 13.80$ & 12.21 & 12.24 & 12.50 & 12.57 \\
\hline
\end{tabular}


star (Moss \& de Vaucouleurs 1986) and must be excluded from the fitting. GR 8 can be considered the most extreme example of an dwarf irregular with mumerous IIII regions and stars "disturbing" the underlying profile. Note the very irregular deviation between the surface brightness profiles of Carignan et al. and ourselves in Figure 2. The close agreement between our total magnitude and that of Carignan $e t$ al. is gratifying and gives us confidence in the results we find in Chapters 4 and 5 using the total magnitudes listed in Table 6 (and 8).

UGC 12151 was observed by de Jong \& van der Kruit (1994), who find $m_{B}=$ $11.13 \pm 0.69$ and $m_{R}=13.75 \pm 0.55$, compared to our $B_{T}^{0}=13.88$ and $h_{T}^{0}=13.52$. They did not correct for galactic extinction $\left(A_{B}=0.21\right)$ so the magnitudes compare even more favorably. McGaugh (1992) (see also McGaugh \& Bothun 1994) observed LGC 5709 and LGC 6151 but list magnitudes from the disk component alone. Since both of these galaxies appear to have rather smooth $B$-band profiles (see Figures 31 and 32), these disk magnitudes can be seen as lower limits and compared with our

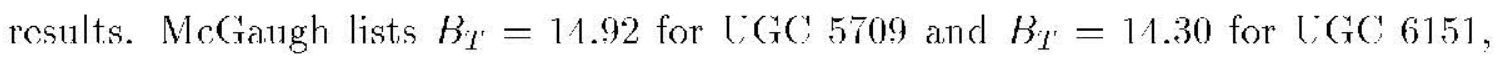
which are very close to our own total magnitudes of 14.98 and 14.65 respectively. The discrepancy would be expected to increase il McGaugh had measured actual magnitudes, but the extreme regularity of the profiles of these two LSB galaxies would tend to minimize this.

T'he average differenee and the deviation are both $\sim 0.2$ magnitudes. Given the differences in technique of the different authors and the irregularity of the galaxies, we feel satisfied with our results. Again, the comparison of the GR8 data is especially reassuring. 


\subsection{Adopted Distances}

A distance for each galaxy in the sample is listed in Table 7. Three important effects must be considered in addition to the basic IIubble expansion when using the heliocentric HI velocitics to obtain distaness. First, the correction for the motion of the sun with respect to centroid of the Local Group from Yahil. Tammann \& Sandage (197T),

$$
\Delta v_{L G}=-79 \cos l \cos b+296 \sin l \cos b-36 \sin b
$$

was applicd, where l and $b$ are the galactic longitude and latitude of the galaxy. Second, the correction for the Virgocentric infall of nearby galaxies based on the Cormulation of Schechter (1980) (see also Aaronson el al. 1982) was applied. A Virgocentric infall velocity of $300 \mathrm{~km} \mathrm{~s}^{-1}$ for the Local Group and a heliocentric velocity of $1026 \mathrm{~km} \mathrm{~s}^{-1}$ for Virgo were adopted. The corrections were calculated with the aid of a program provided by Mark Whittle.

Finally, the Nearby Galaxy Atlas and Catalog (Tully \& Fisher 1987; Tully 1988) were used to determine group membership for each galaxy in the sample, and a mass or luminosity weighted average redshilt for all the galaxies in the group was adopted as the velocity of the galaxy. Because these dwarls are so much less massive than normal galaxics which dominate the groups, the dwarf velocity can include peculiar motions of hundreds of kilometers per second; the weighted average for the group should therefore provide a much more distance-indicative velocity for the dwarf. This corrected velocily is listed in Table 7 .

In the case of galaxies which are not listed in Tully, the Ntas was examined in order to determine likely group membership, based on galactic latitude and longitude 
and systemic velocity, $v_{0}$. This systemic velocity, used by Tully \& Fisher, is simply the heliocentric velocity corrected for the solar motion with respect to the Galaxy, neglecting the Local Group dynamics, and is

$$
\Delta v_{0}=300 \sin l \cos b .
$$

This is similar to the $\Delta v_{L G}$, and varies from it by at most $\sim 90 \mathrm{~km} \mathrm{~s}^{-1}$ in cortain directions. After the group vo was obtained, a correction for the Virgocentric infall was made.

$\Lambda$ IIubble constant of $\mathrm{II}_{0}=75 \mathrm{~km} \mathrm{~s}^{-1}$ was adopted and the distances obtained from the group velocities are listed in Table 5. Two galaxies, UGC 8091 and UGC 9128 (1)I)( 155 and I)I)( 187) are extremely nearby, possible members of the Iocal Group (van den Bergh 1994). Many authors have used various independent distance estimators, including brightest blue and red supergiants and size of the largest HII region to obtain distances to these galaxies. For UGC 8091 these estimates include those from IIoessel \& Danielson 1983; de Vaucouleurs \& Moss 1983; Moss \& de Vaucoulcurs 1986; and Aparicio, García Polayo \& Moles 1988b; for UGC 9128, wo adopt the distance estimate of $1.1 \mathrm{Mpc}$ from Aparicio, García-Pelayo \& Moles $1988 a$.

For the several higher redshift, LSB galaxies, the velocity was corrected for the motion of the local group and the Virgocentric infall, and the distance was obtained in a straightforward way from this velocity.

The distances, listed in Table 7 , were then used to calculate the remaining parameters in the table. "l'hese include the distanee modulus, the disk scale lengths in the $B$ and $/$ from 'Table 5 , converted to kiloparseces and the absolute magnitudes in $B$ and $I$, with $\left(M^{T}\right)$ and without $\left(M^{T-}\right)$ the identified HII regions and stars present int the galaxy. 
Table 7: Distance Dependent Quantities

\begin{tabular}{|c|c|c|c|c|c|c|c|c|c|}
\hline Galaxy & $\begin{array}{r}v_{\text {corr }} \\
\left(\mathrm{km} \mathrm{s}^{-1}\right)\end{array}$ & $\begin{array}{r}\text { D } \\
\text { (Mpc) }\end{array}$ & $\begin{array}{r}m-M \\
(m a g)\end{array}$ & $\begin{array}{l}\alpha_{B} \\
(\mathrm{kp})\end{array}$ & $\alpha_{I}$ & $\begin{array}{l}M_{\mathcal{B}}^{T} \\
(\mathrm{~m}\end{array}$ & $M_{B}^{T-}$ & $\begin{array}{c}M_{I}^{T} \\
\quad(m\end{array}$ & $M_{I}^{T-}$ \\
\hline 31 & 1109.1 & 14.8 & 30.8 .5 & 0.54 & 0.65 & -16.31 & -15.88 & -17.56 & -17.21 \\
\hline 63 & 581.8 & 7.8 & 9.16 & 0.32 & 0.08 & -11.70 & -1.1 .31 & -11.18 & -13.30 \\
\hline 300 & 1183.8 & 1.3 .8 & 0.99 & 1.26 & 0.55 & -15.62 & -13.38 & -15.53 & -15.17 \\
\hline 772 & 876.7 & 11.7 & 0.34 & 0.47 & 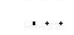 & -16.65 & -16.20 & - & \\
\hline 1171 & 792.1 & 10.6 & & 0.43 & . & -16.75 & -16.37 & $\ldots$ & \\
\hline $\mathrm{K} 10$ & 792.9 & 10.6 & 30.12 & 0.95 & 1.25 & -14.68 & -14.46 & -15.27 & -15.06 \\
\hline $\mathrm{K} 10^{2}$ & 792.9 & 10.6 & & 2.68 & 3.37 & -15.61 & -15.52 & -15.98 & -15.90 \\
\hline 1981 & $1371 . \overline{5}$ & 18.3 & & 0.50 & 0.79 & -15.2 .5 & -11.72 & -16.77 & -16.33 \\
\hline $1981^{2}$ & $137 . .5$ & 18.3 & 31.32 & 1.17 & 0.96 & -16.09 & -16.03 & -16.56 & -16.17 \\
\hline 2017 & 1101.1 & 14.7 & & 1.09 & 0.96 & $-15.6 \overline{0}$ & -15.36 & -16.19 & -15.91 \\
\hline 201 & 1101.1 & 14.7 & & 1.47 & 1.37 & -16.02 & -15.83 & -16.13 & -15.83 \\
\hline 2034 & & 10.2 & & 1.11 & 0.51 & -15.96 & -15.23 & -16.47 & -15.74 \\
\hline & 765.5 & 10.2 & & 1.26 & 0.76 & -16.59 & -16.32 & -16.95 & -16.59 \\
\hline 205 & 40 & & & & 1.08 & -16.82 & & -17.33 & -16.8 .5 \\
\hline 216 & 1039.9 & 13. & & 0.96 & 0.98 & -11.61 & -11.29 & -15.10 & -11.81 \\
\hline - & 1039.9 & 13.9 & & & 2.20 & -14.99 & -14.72 & -15.21 & -15.01 \\
\hline & 2 & 28.4 & & & 1.61 & -22 & -22.10 & -21.04 & -20.45 \\
\hline $\mathrm{I}$ & 1 & 28.4 & & 6.60 & 2.79 & -22.55 & -22.48 & -20.12 & -19.75 \\
\hline K.S & 2 & 2 & & 6.40 & 5.10 & -25 & -2 & -22.45 & -22.18 \\
\hline $321:$ & 1 & & & & 0.60 & -16.67 & -1 & -16.81 & -15.99 \\
\hline 338 & 1395.1 & 1 & & 0. & 0.51 & -15.86 & -1 & -16.82 & -16.20 \\
\hline 381 & & & & 0.37 & 0.38 & -1 & -1 & -15.07 & -14.35 \\
\hline 3860 & 797.9 & 1 & & 0.60 & . & -18.72 & -1 & $\ldots$ & $\ldots$ \\
\hline 3966 & (20) & & & & 1.23 & -15.48 & & -16.11 & -15.63 \\
\hline 4173 & 18818 & 25.1 & & $1.1 \bar{i}$ & 1.38 & -14.69 & -1 & -16.99 & -16.64 \\
\hline $1173^{2}$ & 18818 & $2-1$ & 200 & 2.31 & 2.30 & -17.16 & -17.22 & -17.92 & -17.72 \\
\hline 1204 & 2851.5 & 380 & & & 1.6 .1 & -16.83 & -16.62 & -18.17 & -17.77 \\
\hline M81dw & 3442 & & & & 1.28 & -13.00 & -1 & -15.42 & -15.37 \\
\hline M8 & 12 & & & 7 & 0.86 & -1 & -1 & -13.29 & -13.01 \\
\hline $5423^{2}$ & & & & & 0.21 & 1 & & -14.62 & -14.40 \\
\hline 5706 & & & & & 3.51 & & & & -17.44 \\
\hline 5709 & & & & & 1.75 & & & 90 & -20.81 \\
\hline 61 & & & & & 2.65 & & & & \\
\hline & & & & & 2.98 & -1 & & 19 & .11 \\
\hline 65 & & & & & 3.87 & -1 & & .95 & -18.49 \\
\hline 66 & . & & & 8 & 3.05 & -1 & -1 & -19.59 & -19.50 \\
\hline 662 & & & & & & -1 & & & \\
\hline & & & & & 1.69 & -1 & -1 & -20.15 & -20.09 \\
\hline 754 & & & & & 0.76 & $-1 f$ & -1 & -16.666 & -16.20 \\
\hline 7596 & 1326.0 & I & & & 0.81 & -1 & & -17.29 & -17.06 \\
\hline & & 7.5 & & & 1.38 & -13.29 & -13.06 & -14.99 & -14.92 \\
\hline $7608^{2}$ & 35.1 & & & & $\ldots$ & -16.73 & -1 & $\cdots$ & $\ldots$ \\
\hline 7636 & 1326.0 & & & 2.11 & 2.59 & -16.25 & -1 & -17.85 & -17.83 \\
\hline 7684 & & & & & 0.72 & -15 & -1 & -17.69 & -17.56 \\
\hline 7882 & & .7 & & & 3.81 & -20.07 & -10 & -21.17 & -20.58 \\
\hline 8091 & 1326.0 & 1.1 & & 0.08 & 0.11 & -10.68 & -9.78 & -11.57 & -11.00 \\
\hline $8201^{2}$ & & & & & $\ldots$ & -15.12 & & & $\ldots$ \\
\hline & & & & & 1.11 & & & & -15.41 \\
\hline 8760 & .3 & & & 0.26 & 0.35 & -1 & - & 60 & -14.09 \\
\hline & & & & & 0.40 & -1 & & 49 & -14.15 \\
\hline & & & & & 0.10 & -1 & & .35 & -11.71 \\
\hline 93 & & & & & 2.89 & -18.12 & -1 & -19.58 & -19.11 \\
\hline & 5 & & & 1.05 & 1.87 & -15.12 & - & -16.50 & -17.69 \\
\hline & -8 & & & 175 & 3.23 & -17.22 & -16.72 & -18.06 & -19.23 \\
\hline & & 31 & 0 & 2.46 & 5.96 & -18.94 & -1 & -20.46 & -21.46 \\
\hline & 370 & & & 2.43 & 2.00 & -1 & -1 & -18.32 & -19.65 \\
\hline 106 & & & & 0.75 & 0.79 & $-14.7 \mathrm{t}$ & $-14.5 i$ & -15.21 & -16.53 \\
\hline 11764 & 3603.4 & 48. & . & 2.80 & $\cdots$ & -17.75 & -17.38 & .. & \\
\hline $12082^{2}$ & 1091.2 & 1.1 .5 & 30 & 1.97 & 2.20 & -17.03 & -16.56 & -18.16 & -17.60 \\
\hline 12151 & 1798.6 & 21.0 & 31.90 & 2.67 & & -18.02 & -17.68 & $\cdots$ & \\
\hline 12894 & 595.5 & 7.9 & 29.50 & 3.38 & 2.31 & -17.29 & -17.26 & -17.00 & -16.93 \\
\hline
\end{tabular}


Table 8 contains the $R$-band disk and magnitude information which can not be found in the three previous tables.

Table 8: h-band Photometric Results

\begin{tabular}{lrrrrrrrrrr}
\hline \hline Galaxy & $\begin{array}{r}\mu_{R}^{0} \\
\left(\frac{\mathrm{mag}}{\operatorname{arcscc^{2}}}\right)\end{array}$ & $\begin{array}{r}\alpha_{l i} \\
\left({ }^{\prime \prime}\right)\end{array}$ & $\begin{array}{r}R_{\infty} \\
(\mathrm{mag})\end{array}$ & $\begin{array}{c}\mu_{1 i}^{(0.6)} \\
\left(\frac{\mathrm{mag}}{\operatorname{arcscc^{2}}}\right)\end{array}$ & $\begin{array}{c}R_{25} / R_{26} \\
(\mathrm{mag})\end{array}$ & $\begin{array}{c}R_{T}^{0} \\
(\mathrm{mag})\end{array}$ & $\begin{array}{c}R_{T^{0}}^{0-} \\
(\mathrm{kpc})\end{array}$ & $\begin{array}{c}\alpha_{R} \\
(\mathrm{mllag})\end{array}$ \\
\hline 10031 & 22.82 & 11.1 & 15.85 & 23.23 & $15.12 / 15.25$ & 15.19 & 15.75 & 0.99 & -16.09 & -15.53 \\
10058 & 22.09 & 7.9 & 15.65 & 22.19 & $15.16 / 15.04$ & 15.02 & 15.60 & 1.27 & -17.58 & -17.00 \\
10290 & 22.20 & 18.2 & 13.93 & 22.31 & $\ldots / \ldots$ & 13.01 & 13.78 & 2.73 & -19.51 & -18.74 \\
10376 & 21.96 & 6.9 & 15.91 & 22.20 & $15.11 / 15.37$ & 15.32 & 15.86 & 1.66 & -18.15 & -17.61 \\
10669 & 22.12 & 8.0 & 15.83 & 22.45 & $15.73 / 15.58$ & 15.51 & 15.75 & 0.49 & -15.01 & -14.77 \\
11761 & 19.63 & 5.0 & 11.11 & 19.65 & $15.21 / 15.11$ & 15.11 & 15.59 & 1.18 & -18.30 & -17.82 \\
12151 & 22.00 & 15.7 & 14.29 & 22.38 & $13.64 / 13.56$ & 13.52 & 13.91 & 1.82 & -18.38 & -17.99 \\
\hline \hline
\end{tabular}

\subsection{HI Linewidth Corrections}

The galaxics in this sample have been chosen to lie in the turbulenes dominated regime, and are therefore distinct from previous Tully-Fisher samples. As a first attempt to correct for turbulence, and in order to be consistent with previous work, however, we calculate a turbulence corrected III linewidth, following Tully \& Fouqué (1985). This formulation yiclds a smooth transition betwen the case of linear summation of the rotational and dispersional motions found for giant galaxies and quadratic summation of the terms, which is observed to apply for dwarf galaxies (Tully el al., 1978):

$$
W_{r}^{2}=W_{20}^{2}+W_{t u r b}^{2}-2 W_{20} W_{t u r b}\left[1-e^{-\left(W_{20} / W_{c r i t}\right)^{2}}\right]-2 W_{\text {lurb }}^{2} e^{-\left(W_{20} / W_{c r i t}\right)^{2}} .
$$

Here $W_{20}$ is the observed line width at $20 \%$ of maximum ( $\Delta v_{20}$ in Table 1$), W_{\text {turt }}$ is a turbulent width which is equal to $2 k \sigma_{z}$, which is equal to $38 \mathrm{~km} \mathrm{~s}^{-1}$, with $k=1.89$ (the factor which yields the $20 \%$ width of a Gaussian profile) and with 
$\sigma_{z}=10 \mathrm{~km} \mathrm{~s}^{-1}$, based on observations of face-on spirals (t.g., van der Kruit \& Shostak, 1982). 'T'his value for the magnitude of the turbulent component agrees with aperture synthesis observations of dwarf galaxies (Tully et al., 1978; Lo et al. 1993). $W_{c r i t}$ defines the transition region between Gaussian profiles and doublehorned profiles, and is taken to be $120 \mathrm{~km} \mathrm{~s}^{-1}$. The rotational line width, $W_{r}$ must still be corrected for inclination, therefore

$$
W_{r}^{i}(\mathrm{TFq})=W_{\mathrm{r}} / \sin \mathrm{i}
$$

where we indicate that we have adopted the corrections of Tully \& Fouqué by the designation ('l' $\mathrm{F} q$ ).

When $W_{20}$ becomes smaller than $W_{t u r t}$, $W_{\text {Rot }}=0$. 'This is simply the point where rotation becomes unimportant and the observed linewidth becomes dominated by turbulence (the exact transition point is of course defined by the choice of $W_{\text {turb }}$ ). Tully \& Fouqué therelore define a dynamical line width:

$$
W_{\nu}^{i^{2}}=W_{r}^{i^{2}}\left(\mathrm{~T}+\mathrm{q}_{1}\right)+1 \sigma^{2}
$$

where the $4 \sigma^{2}$ term is a pressure term. In this way, in the case of spherical symmetry and uniform density, the mass interior to $R$ is $W_{D}^{i^{2}} R / 4 G$ in the case of either pure circular motion or purc random motion. Herc we adopt $\sigma=17 \mathrm{~km} \mathrm{~s}^{-1}$, by assuming $\sigma_{x}=\sigma_{y}=\sigma_{z}=10 \mathrm{~km} \mathrm{~s}^{-1}$ (de Vaucouleurs, de Vaucouleurs \& Buta 1983). This dynamical linewidth should serve as a better indicator of the total mass of the galaxy and should therefore be better suited for use in the Tully-Fisher relation.

We will find these rather simple corrections for turbulence to be inadequate for the interpretation of our data in the next chapter. 'l'he linewidth correction which we finally adopt is further modified, to model more realistically in individual dwarf galaxies the ratio of turbulent to rotational motion. 


\section{Chapter 4}

\section{Tully-Fisher Relation for Dwarfs with Narrow HI linewidths}

\subsection{Tully-Fisher Relation for Normal Galaxies}

\subsubsection{Historical Background and Theoretical Basis}

The obscrved relationship between the $21 \mathrm{~cm} \mathrm{HI} \mathrm{linewidth} \mathrm{and} \mathrm{the} \mathrm{absolute} \mathrm{magni-}$ tude of a galaxy, known as the T'ully Fisher or luminosity linewidth relation, is one of the most widely used distance indicators for galaxies (see Jacoby et al. 1992, for a recent review). The relative ease of measuring global III profiles and obtaining total magnitudes of gas-rich spiral galaxies make this a particularly straightlorward distance indicator. This can be seen when the method is contrasted with the primary distance indicator for ellipticals, the Fundamental Plane relationship (Dressler et al. 
1987 ) between velocity dispersion, effective surface brightness and effective radius which arose as an extension of the Faber-Jackson (1976) relation.

As lar back as the early 1960's (Dieter 1962; Roberts 1962a, 1962b), it was realized that the relationship between the total mass and the III properties (III flux, HI mass fraction, angular size, and HI linewidth) of a galaxy could be used to obtain the distance. Brosche (1971) pointed out a Hubble type dependence on the mean observed $V_{\max }$ : while Rogstad \& Shostak (1972) noted a tight correlation between $V_{m a x}$ and the optical radius lor a sample of Scd galaxies, and suggested that the global propertics of galaxies could be completely described by the Hubble type and $V_{\text {max }}$, but did not propose this as a method of obtaining the distance.

Balkowski et al. (1974) were the first to clcarly state that "within a given morphological type, $W_{t}$ [the linewidth corrected for inclination] is correlated to the luminosity". They also noted an extension to dwarl galaxies of the diameter-luminosity relation found for spiral galaxies by IIeidmann (1969).

It was not until the work of Tully \& Fisher (1977); however, that the usefulness of the HI linewidth as a distanee indicator was fully realized. They pointed out the extreme importance of having calibrators with well determined distances to reduce the scatter in order to make the relationship useful (or indeed even recognizable, in the first instance) as a distance indicator. They found a relationship of the form:

$$
L \propto \Delta V_{0}^{2.5 \pm 0.3}
$$

which corresponds to

$$
M_{p g} \propto-a \log \Delta V_{0}^{\gamma}-b
$$

Tully \& Fisher give a simple physical argument to justily this relation; mannely, the 
total mass of the galaxy (from the virial theorem) is given by

$$
M_{T} \propto R \Delta V_{0}^{2}
$$

and if the $M / L$, ratio is assumed to be constant and the empirical relation of $I \times K^{2.8}$ from Heidmann (1969) for Virgo cluster spirals is adopted, then

$$
L \propto \Delta V_{0}^{3.1}
$$

The work of 'Tully \& Fisher was done using photographic magnitudes, corrected for galactic extinction and inclination of the galaxy; and HI line widths, also corrected for inclination (but in the opposite sense: magnitudes are corrected to "Cace-on", while inclinations are corrected to "edge-on"). This method was immediately seized upon by Sandage \& T'ammann (1976) as way to test other methods of distanec determination.

The first major improvement in this technique came about with the use of infrared $H$-band magnitudes (instead of photographic or photoelectric visual magnitudes) by Aaronson, IIuchira \& Mould (1979), who found a tighter relationship (lurther improved in Aaronson el al. 1982). This improvement was possible because the (uncertain) correction for extinction is much smaller in the infrared (Aaronson ct al. apply no correction), and the luminosity is dominated by late-type giants in the infrared. This presumably would make the assumption of $M / L=$ constant valid for all galaxies, regardless of type. The dispersion in the $I I$-band relation is $\sim 0.25$ mag (Picrec \& 'Tully 1988).

Despite the hope of "lully \& Fisher (1977) that there should be "modest, if any, type dependence" in the relation, Roberts (1978) found a strong type dependenec for the linewidth at a constant absolute magnitude. This result was systematically confirmed by Rubin $e l$ al. (1985) in both $B$ and $I I$. This type dependence manilests 
itself as a zeropoint shift in the TF relation for different types. This would cause a smaller value for the slope to be measured, because of the type dependence of $\Delta V$. Rubin el al. found a slope for the TF relation of -10 lor each type (Sa, Sb, Sc) considered separately.

Aaronson et al. (1979) outlined the physical basis upon which one would expect a slope of -10 for the TF relation (although they found a slope of $\sim-9.5$ in the $H$-band, a discrepancy which is attributed by Rubin $e t$ al. to the dependence on type). This slope implies

$$
L \times V_{\max }^{1}
$$

which is also found to hold for ellipticals through the Faber Jackson relation when $V_{\max }$ is replaced with the velocity dispersion $\sigma$ (Faber \& Jackson 1976). Aaronson et al. state that such a power law will naturally arise, from the virial theorem if three conditions are true: "(a) all galaxies have the same mass profiles and rotation curves as a function of some dimensionless scale length, (b) all galaxies have the same central mass surface density, and (c) all galaxics have the same mean $M / L$."

Conditions (b) and (c) imply constant central surface brightness $\mu_{0}$ (Burstein 1982), which is not observed over a wide range in galaxies (Binggeli 1994, Ferguson \& Binggeli 1994). This is in marked contrast to the earlier work of Freeman (1970), who found a constant $\mu_{0}$ for spiral galaxies (but see Bosma \& Freeman 1993). Ilowever, a requirement of constant surface brightness averaged over the disk can also be secn to imply the power law result. If surface brightness is constant $\left(L \propto R^{2}\right)$ then the virial theorem becomes:

$$
M_{w i r} \propto L^{0.5} V_{\max }^{2}
$$

which yields the $L \propto V_{\max }^{4}$ power law result if $M / L$ is constant. 
It is interesting to note that if $M / L \propto L^{\gamma}$ and is not constant, then the relation becomes:

$$
L \propto V^{1 /(2 \gamma+1)_{m a x}}
$$

In addition, if $I \propto K^{(2+e)}$ so that neither surface brightness nor the mass to light. ratio are constant, then

$$
L \propto V_{m a s}^{\frac{4+c}{2 \gamma+c \gamma+c+1}}
$$

The slope for this modified TF relation would then be $\left(-\frac{10+5 \epsilon}{2 \gamma+\gamma t+t+1}\right)$, as opposed to $-10$.

\subsubsection{Nonlinearity of the Tully-Fisher relation}

We have already noted the effect that the dependence of $M / L$ on Hubble type has on the zeropoint of the TF relation, which is actually observed as a shallower slope when the sample is not sorted by type. More recently authors have noted that the relation appears to exhibit curvature, in the sense that galaxies at both extremes in luminosity appear to be underluminous at a given linewidth, when compared to the linear TF relation (Aaronson el al. 1982; Aaronson $t$ al. 1986). The departure at the faint end was attributed at least in part to nomrotational (or turbulent) motion broadening the HI linewidth (Hoffman, Helow, \&. Salpeter 1988). However applying a simple turbulent width correction as given by Bottinclli et al. (1983) or 'lully \&. Fouqué (1985) does not entirely remove this deviation. For the brightest spirals, a relationship of decreasing metallicity with luminosity (Bothun el al. 1984) would cause these objects to appear underluminous lor a given mass.

The possibility that observational sources of error have given rise to this nonlincarity has becn investigated in depth (Aaronson \& . Mould 1986; Bothun \&. Mould 
1987; Pierce 1988; Kraan-Korteweg, Cameron, \& Tammann 1988; Burstein \& Raychaudhury 1989), and while there is still a lively debate over the impact of Malmquist bias on samples used to determine the IIubble Constant (Pierce \& Tully 1992; Sandage 1991a \& 1994 b and references thercin), this secms unlikely.

I'heoretical interpretations of the nonlinearity have pointed to dependenee on the environment (and therefore the formation) of the galaxies (Djorgovski, de Carvalho, \& Han 1988), as well as a luminosity dependence of the dark matter content of disk galaxies (Persic \& Salucci 1988, 1990; Mould, IIaIı, \& Bothun 1989; Pierce 1991). 'The use of the T'F relation to measure the dark matter content of galaxies is potentially one of the most powerful applications of this relation, aside from its primary utility as a distance indicator.

\subsection{Motivation for this Work}

Why should we examine the Tully-Fisher relation in dwarf galaxies? There are three main reasons, in light of the preceding discussion of the TF relation, for examining it in detail for dwarf galaxics.

First, is the curvature at the faint end real, and can it be attributed entirely to t.urbulenec? If so, is there a way to still use a modified line width as an indicator of the mass? That is, is there a correction of the form used by Bottinelli et al. (1983) or Tully \& Fouqué (1985), which will allow the raw III linewidth to be converted to a parameter which measures the mass, even in dwarl galaxies?

Second, we wish to determine the uselulness of the TF relation as a distance indicator for dwarfs. If the curvature can be removed, what is the remaining dispersion 
at the faint end? If it is small enough, the TF relation applied to dwarfs would be useful for determining the distances to nearby clusters because of the overwhelming numbers of dwarls. Measurements of the III linewidths already exist for a large number of dwarf galaxies (Fisher \& Tiully 1981: Schncider et al. 1990, 1992), which can be potentially useful for mapping bulk flow velocities. The Hubble type dependence for the TF relation among spirals (Rubin et al. 1985) limits the number of galaxies in a cluster which can be used to obtain a distance.

Finally, what can be determined about the dark matter content of dwarls using the 'T'F relation (Picre 1991)? Is there an indication of increased dark matter content in dwarfs as compared to more luminous galaxies? If so, this would be a clue to possibly different formative processes for dwarf and normal galaxies.

In order to address these questions, we must first select a sample of galaxies which are appropriate for an investigation of the TF relation.

\subsection{Tully-Fisher Relation for the Sample}

\subsubsection{The Basic Relation}

As a first attempt at examining the relationship, we will simply plot the linewidth versus the absolute magnitude for all of the galaxies for which we have photometric data. We will exclude the galaxies observed under non-photometric conditions, and those which were too large to fit entirely on the CCD chip. Also excluded are UGC 7636 , sine the $\mathrm{HI}$ detected has been stripped from the dwarf (Patterson \& 'I'huan 1992; McNamara ot al. 1994), and therefore the linewidth will not be indicative 
of the mass. Finally, we exclude Karachentseva 37 and $37 \mathrm{C}$ (IC342dw6), since the detected III profile is expected to be the sum of the contribution from each of the galaxies, and not to either one alone.

In Figure 81(a), the linewidth, $W_{20}$, without any corrections for inclination or turbulenec is used; while in Figure $81(\mathrm{~b}), W_{r}^{i}\left(\mathrm{l}^{\prime} \mathrm{Fq}\right)$, the linewidth corrected as described in Chapter 3 (equation 3.18) following the method of Tully \& Fouqué (1985), is plotted.

The LSB galaxies from our sample are plotted as filled squares, while the data for the "narrow-lined dwarl" sample are plotted as filled triangles. Galaxies from the May85 obscrving run, marked with an $r$ in 'lable 1 (Chapter 1), are assigned to the ISB category if they have large linewidths (UGC 10058, 10290 and 12151), and into the narrow-lined dwarf category if they have narrow line widths and a small redshilt (LGC 10031 and 10669). The two intermediate cases, UGC 10376 and 11764 (intermediate because while they have lairly narrow linewidths, $\Delta V_{20}=69$ and $107 \mathrm{~km} \mathrm{~s}^{-1}$ respectively, they are at $v_{0}>3000 \mathrm{~km} \mathrm{~s}^{-1}$ ) are marked by open circles.

The Pierce \& Tully (1992) spiral galaxies are plotted as open squares. In Figure $81(b)$ the TF relation from Pierce \& Tully (1992) (which included galaxies in the Local, Sculptor, and M81 groups using 6 local calibrator galaxies with independently determined distances) is shown as a solid line and the relation from Picrec \& 'Tilly (1988) (using Virgo and Lirsa Major cluster galaxics and 3 local calibrator galaxics) as a dotted line for comparison, in order to show some of the observed range in the TF relation. The $I$-band relations are shown in Figures 82(a) and (b), with the data from the same sources and plotted with the same symbols. 


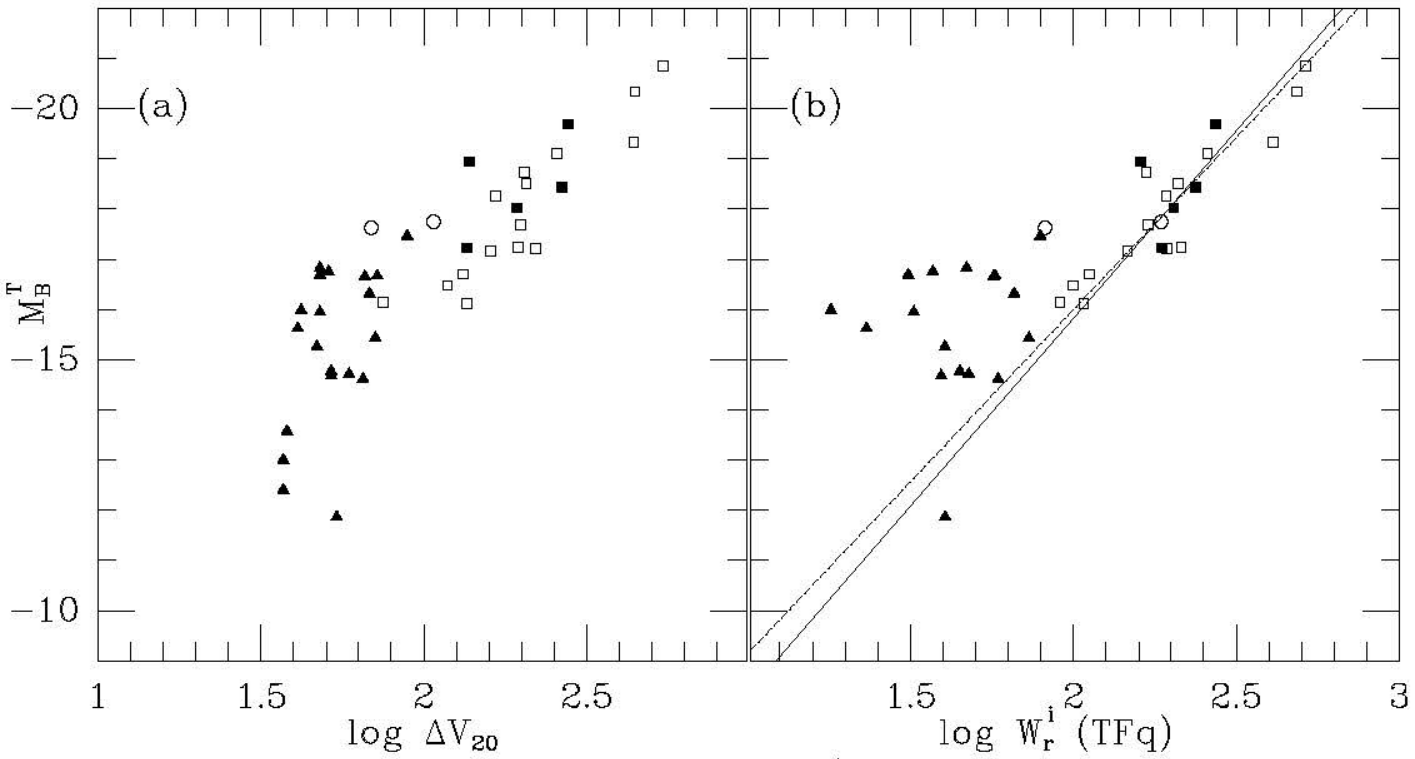

Figure 81: $B$-band Tully-Fisher Relation. (a) is a plot of the observed linewidth versus the absolute magnitude, while (b) shows the corrected line width (equation 3.18). The open squares are the galaxies from Pierce \& Tully (1992), the filled squares are the LSB galaxies from this work, the filled triangles are the marrowlined dwarfs from this sample, and the two open circles are intermediate cases. The solid line in (b) is the empirical Tully-Fisher relation from Pierce \& Tully (1992) and the dashed line is from Picrec \& Twlly (1988). 'The 3 galaxies plotted at the extreme lover left in (a) have a raw linewidth of less than $38 \mathrm{~km} \mathrm{~s}^{-1}$ and therefore a corrected linewidth of 0 . They are not plotted in (b).

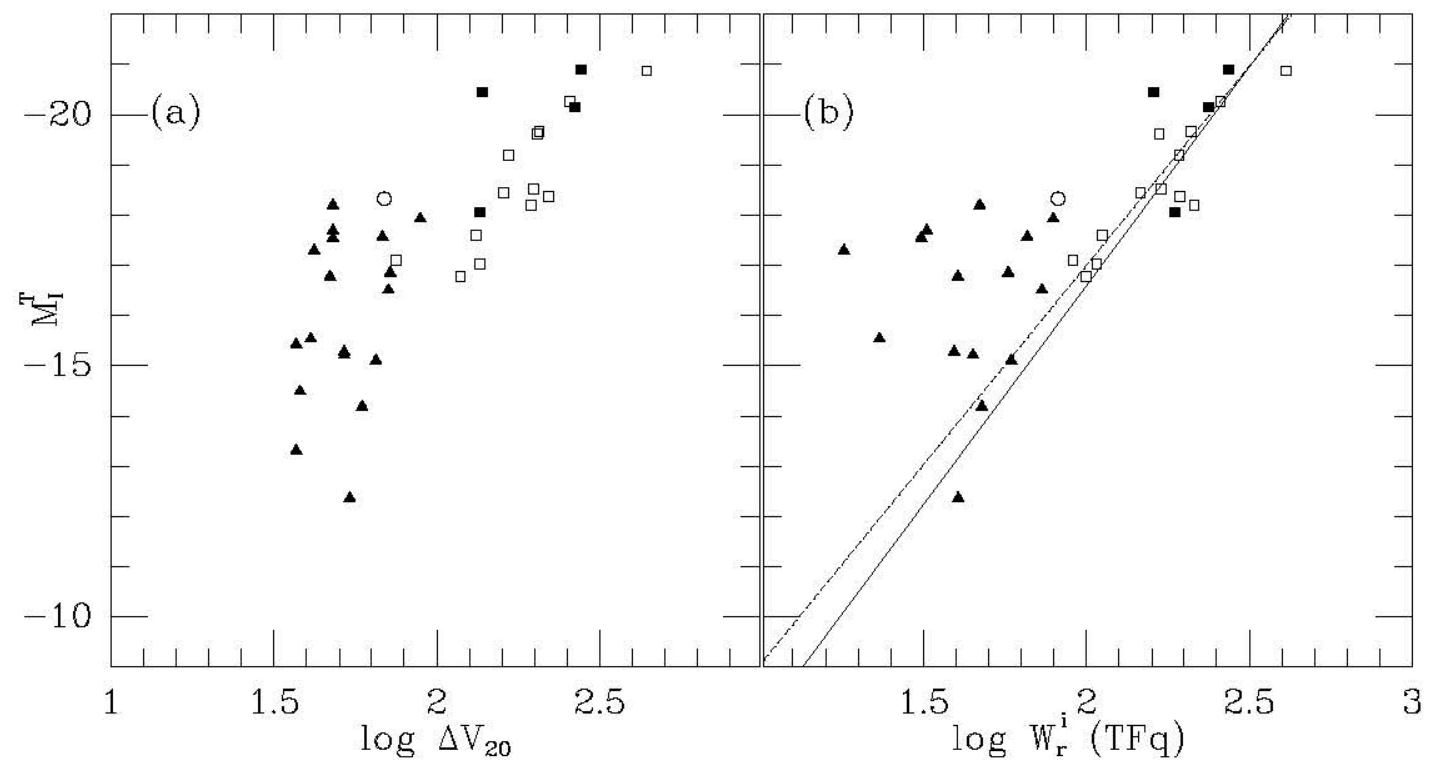

Figure 82: $I$-band Tully-Fisher Relation. Same as Figure 81 for the $I$ data. 
It is clear that the LSB galaxies from the sample fit the TF relation very well, while the narrow-linewidth dwarfs do not, either before or alter correction for the inclination and turbulence. It appears possible that the dwarl linewidths have been "overcorrected", which is possible if either the inclination correction is too large or the simple correction applied for the turbulence is inappropriate.

It should be stressed that the turbulent correction could be wrong for all the galaxies: however, a slight error would be easily visible only in the narrow-lined dwarls with $\log \Delta V_{20}$ close to $1.58\left(\log 38 \mathrm{~km} \mathrm{~s}^{-1}\right)$. The abrupt fallofl of the relation shown in Figure 81(a) very close to this value indicates that there is indecd a turbu-

Ient limit near $38 \mathrm{~km} \mathrm{~s}^{-1}$ ), but does not indicate that the Tully Fouque correction is necessarily of the correct form.

\subsubsection{Correlations with dispersion}

In order to investigate possible causes of the dispersion in the 'T' 1 ' relation at the faint end, we examined the correlation of the residual from the Pierce \& Tully (1992) relation with various observables.

In Figure 83, the TF residual.

$$
\text { TF res }=M_{B}^{Y-}-7.48 \log W_{r}^{i}(\mathrm{TFq})+0.85
$$

is plotted against the derived inclination, the "HII ratio", the color, the measured Holmberg radius, the absolute magnitude, the HI mass, and the profile shape. If the deviation from the relation is due to observational error, one would expect the inclination to be the most likely source of this error, because the correction of the linewidth to edge on depends eritically on the inclination. No correlation is apparent 

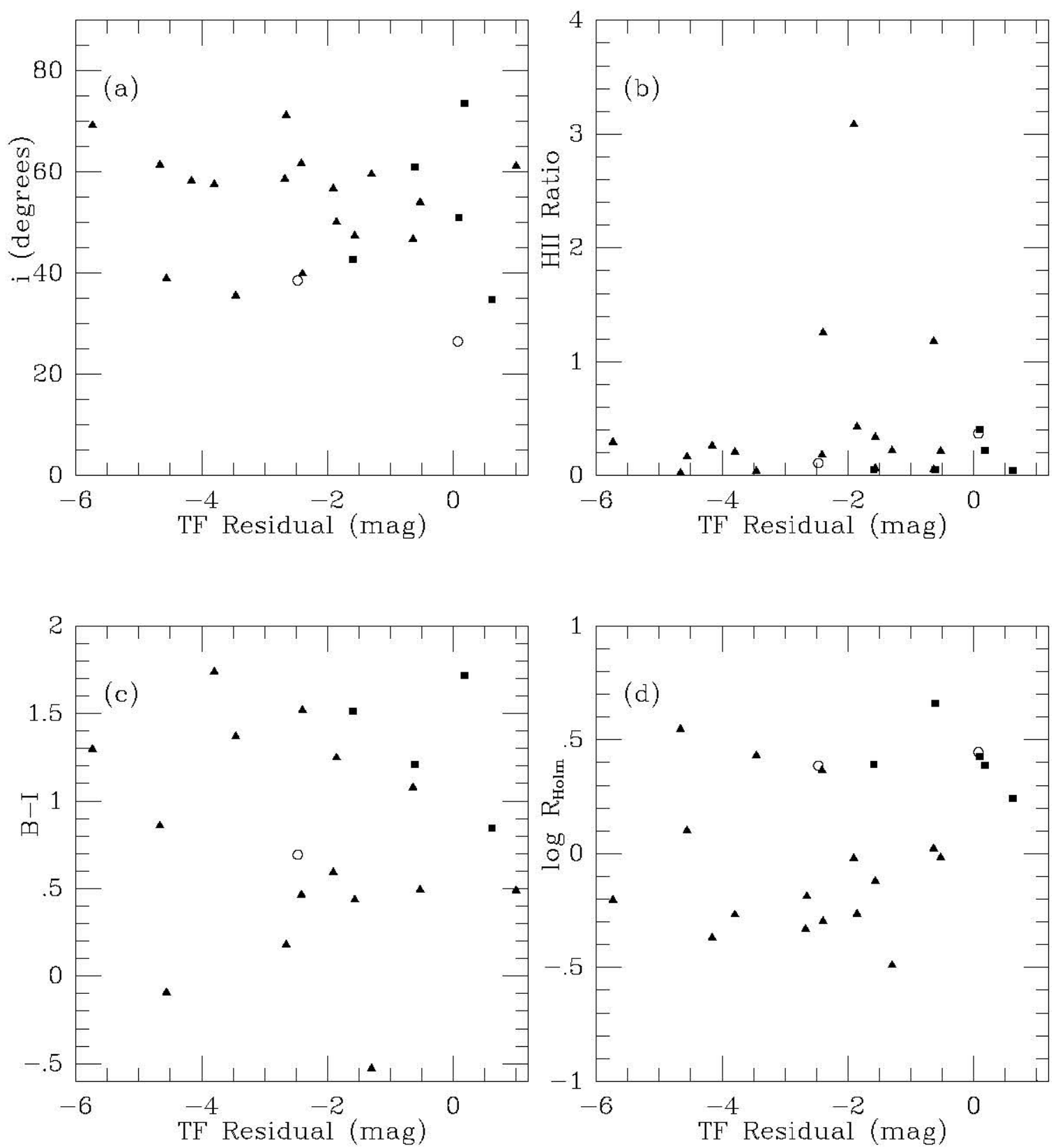

Figure 83: Plots of the Residual from the 'T'F' relation (equation 1.9) versus various obscrvables. Symbols are the same as in Figure 81. (a) T'F' Residual versus inclination. A correlation would be expected if there was a systematic cror in the inclination, which would effect the linewidth through equation (3.18). (b) Residual versus HII Ratio (sce text). (c) Residual versus color and (d) Residual versus the optical (Holmberg) radius of the galaxy. No correlation is apparent with any of these parameters. 

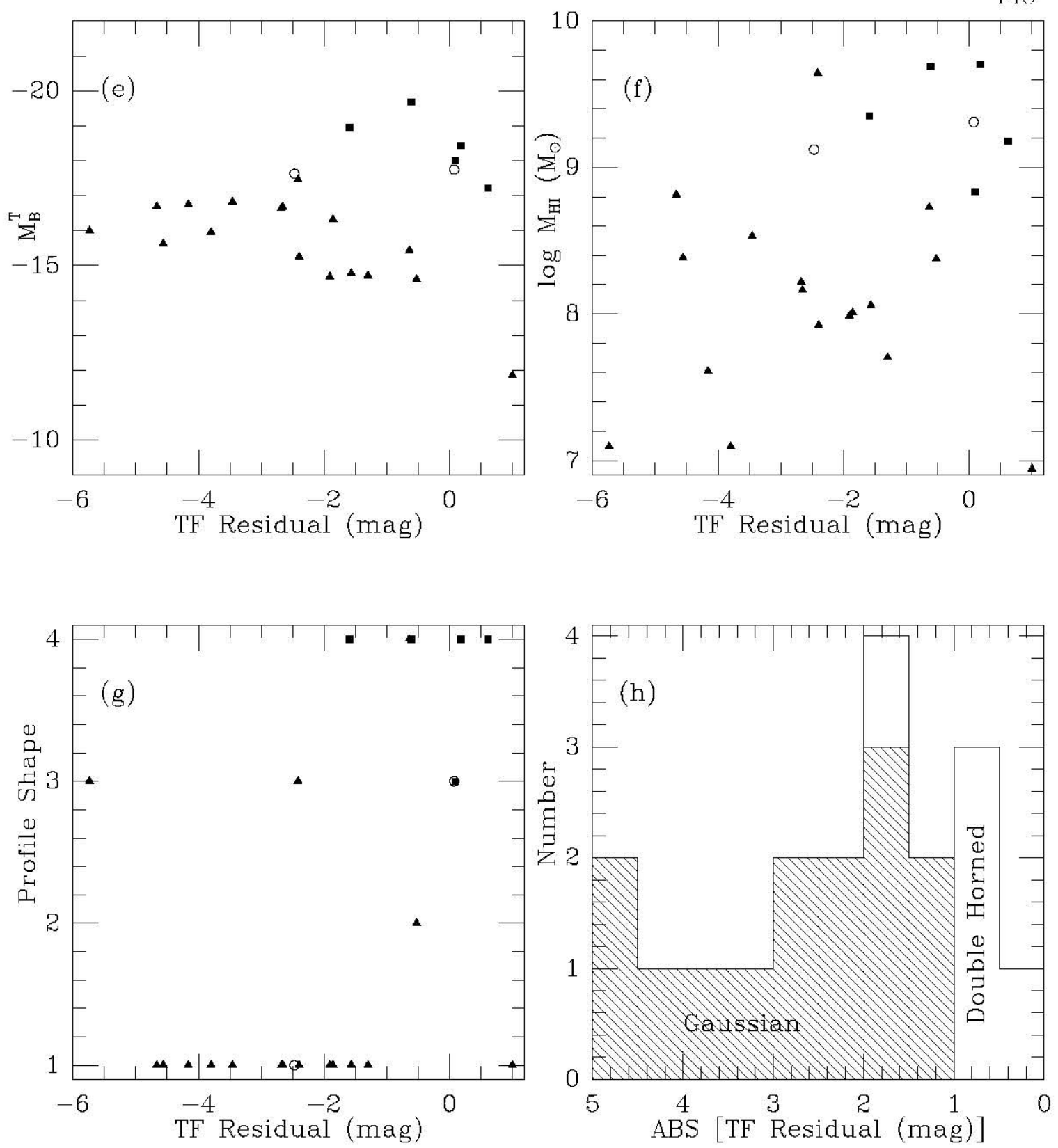

Figure 83: (cont) Plots of the Residual from the 'l'F relation versus various observables. (c) Residual versus absolute magnitude: compare with Figure 81(b). (f) Residual versus HI mass; a weak correlation is present, indicating that the deviation from the 'l'F relation is related to the HI, rather than optical, properties of the galaxy. (g) Residual versus HI Profile Shape. 1 indicates a definite Gaussian profile; 1 a definite 1)ouble. Horned profile, while 2 and 3 indicate profiles which are intermediate or uncertain. A clear difference between the residual for 1 and 4 is present. (h) Histogram for the absolute value of the TF residual for Gaussian (hatched) and Double Horned Protiles. 
(Figure 83(a)).

The "HII index" which is a measure of the area of the galaxy excluded from the total magnitude (because of IIII regions or stars), normalized by the total area of the galaxy, might be expected to correlate with the residual if there is some systematic problem with the total magnitudes we are 11sing which is eaused by the exclusion of the HII regions and stars. It would also indicate a dependence of the TF residual on increased star formation in a galaxy, which would be expected if the star formation rate was high enough to disturb the global III profile, through the effects of supernovar (Westpfahl \& Puche 1994). The $B-/$ color might be expected to correlate for a similar reason. In neither case does a correlation appear to be present (Figure 83(b).(c)).

The Holmberg radius is plotted versus the residual in Figure 83(d). The absolute magnitude is plotted in Figure 83(e). As can also be seen in Figure 83(b), the eflect is not linear with magnitude, though the dispersion does increase at lower luminosities. This is merely a consequence of the increased scatter at the faint end.

I'he trend with $\log M_{H 1}$ which can be scen in Figure $83(\mathrm{f})$ indicates at least that the dispersion is related to the HI properties of the sample, and not to the optical properties (or any errors in the measurement of these properties).

Finally, the most dramatic relation is that between the TF residual and the HI profile shape (Figure 83(g)). The profiles have been classified in Table 1, as Gaussian (=1), probably Gaussian (=2), probably I)ouble Horned (=3), and I)ouble Horned (=1). If the uncertain classifications are excluded, the dichotomy of the two classes of profiles can be clearly seen (Figure $83(\mathrm{~h})$ ). Since the Gaussian profiles are expected when the motion is largely (but not exclusively) turbulent, while the Double IIormed 
(DH) profiles are found when rotation dominates, the linewidth is at the very least measuring the mass in a different way in the two cases.

This correlation, then, is what one would expect even in the absence of any observational errors. The fact that the galaxies with Gaussian profiles exhibit such large residuals even after they have been corrected for turbulent motion, confirms the suspicion that the Tully-Fouque correction for turbulence is not adequate.

\subsection{Modified Version of the Line Width}

In place of the assumption that all galaxies have a turbulent component of $38 \mathrm{~km} \mathrm{~s}^{-1}$ : we follow Staveley-Smith el al. (1992), and assume that the ratio of rotation to dispersion, 3 , in a dwarl galaxy is given by the intrinsic axial ratio, qo (equation 3.5) in the following manner (Binney, 1978; Fall \& Frenk 1983):

$$
\beta=\left(\frac{V_{r o t}}{\sigma}\right)^{2}=\frac{\left(1+2 q_{0}^{2}\right) \cos ^{-1} q_{0}-3 q_{0}\left(1-q_{0}^{2}\right)^{1 / 2}}{q_{0}\left(1-q_{0}^{2}\right)^{1 / 2}-q_{0}^{2} \cos ^{-1} q_{0}} .
$$

which follows from the tensor virial theorem (Binney, 1978) and holds for an oblate cllipsoidal disk with a negligible contribution to the apparent axial ratio from a bulge component. Here $V_{\text {rot }}$ is the maximum rotational velocity and $\sigma$ is the velocity dispersion. We can then replace $W_{t}$ in equation (3.17) with $3.5882 \sigma$ (the factor of 3.5882 converts from $\sigma$ to full width at $20 \%$ of the peak for a Caussian) to obtain:

$$
\begin{aligned}
W_{r}^{i^{2}}(\text { quad }) \sin ^{2} \mathrm{i}= & W_{20}^{2}+(3.5882 \sigma)^{2}- \\
& 2 W_{20}(3.5882 \sigma)\left[1-\epsilon^{-\left(W_{20} / 120\right)^{2}}\right]- \\
& 2(3.5882 \sigma)^{2} c^{-\left(W_{20} / 120\right)^{2}}
\end{aligned}
$$


which is simply equation (3.17) with a factor of $\sin ^{2} i$ included to remove the effects of inclination, and $W_{c r i t}=120 \mathrm{~km} \mathrm{~s}^{-1}$. Since $W_{r}^{i}=2 V_{\text {rot }}$ (even lor dwarl galaxies; Tully el al. 1978), we find after substituting $\sigma=W_{r}^{i}($ quad $) /(2 \sqrt{\beta})$ that,

$$
\begin{aligned}
0= & W_{r}^{i^{2}} \text { (quad) }\left[\sin ^{2} \mathrm{i}+\left(\frac{3.5882}{2 \sqrt{\beta}}\right)^{2}\left(2 \mathrm{e}^{-\left(\mathrm{W}_{20} / 120\right)^{2}}-1\right)\right]+ \\
& W_{r}^{i} \text { (quad) }\left[\frac{3.5882 \mathrm{~W}_{20}}{\sqrt{\beta}}\left[1-\mathrm{e}^{-\left(\mathrm{W}_{20} / 120\right)^{2}}\right]\right]-\mathrm{W}_{20}^{2}
\end{aligned}
$$

which is a simple quadratic in $W_{r}^{i}$ (quad).

A further assumption is introduced when we consider that the corrected linewidth is correlated with the absolute magnitude presumably because the mass is correlated with the luminosity, and the linewidth is related to the mass through the virial theorem (sce discussion in \$1.1.1). The virial theorem in solar units is

$$
M_{v i r}^{r o t}=2.33 \times 10^{5} r_{v i r} V_{r o t}^{2} \text { : }
$$

or

$$
M_{v i r}^{r o t}=2.33 \times 10^{5} r_{v i r}\left(\frac{W_{r}^{i}\left(\mathrm{~T}^{\prime} \mathrm{F}_{\mathrm{q}}\right)}{2}\right)^{2},
$$

with $r_{\text {vir }}$ the virial radius (usually taken to be the Holmberg radius for dwarfs and $R_{25}$ lor spirals) in kiloparsecs, and $V_{\text {rot }}$ and $W_{r}^{i}(\mathrm{TFq})$ are given in $\mathrm{km} / \mathrm{s}$. The rol indicates that this mass is calculated assuming rotation alone (except for the constant additive factor of $38 \mathrm{~km} \mathrm{~s}^{-1}$ ).

However, if there is a significant contribution from random motions then this must be generalized to (Staveley-Smith et al. 1992):

$$
M_{v i r}^{\text {rot }+ \text { turb }}=2.33 \times 10^{3} r_{v i r}\left(3 \sigma^{2}+V_{r o t}^{2}\right)
$$

or

$$
M_{v i r}^{r o t+t u r b}=2.33 \times 10^{5} r_{v i r}\left(\frac{W_{r}^{i}(q u a d)}{2}\right)^{2}(1+3 / \beta) .
$$


It would be equivalent to substitute

$$
W_{r}^{i^{2}}(\bmod )=W_{r}^{\mathrm{i}^{2}}(\text { quad })(1+3 / \beta)
$$

for $W_{r}^{i^{2}}$ in the Tully Fisher relation (with $W_{r}^{i^{2}}$ (quad) obtained from solving the quadratic in equation (4.12)), if we still wish to plot the luminosity versus a "linewidth". We plot the modified linewidth versus the absolute magnitude in Figure 84, with the Pierce \& Tully galaxies again represented by open squares. In this case we plot only the galaxies from our sample which are have profiles which are either clearly Gaussian (filled triangles) or clearly Double Horned (I)H; filled squares).

The most striking feature of this plot is the dichotomy between the Gaussian and DH galaxies. The overall appearance of the relation is much closer to that for the raw linewidths (Figure 81(a)) than for the Tully-Fouqué modified line widths (Figure 81(b)). There is clearly a difference in the $M / L$ dependence on $L$ between the Gaussian and the 1$) \mathrm{H}$ galaxics. The transition occurs at $M_{B}^{T} \sim-16$.

At this point it might be more informative to plot the luminosity versus the virial mass (from equation 4.16) directly, instead of "hiding" the mass inside this modified linewidth, $W_{r}^{i}(\bmod )$. This is shown in Figure 85(a) for the same points as in Figure 84.

\subsection{Turbulence Alone?}

Is it really necessary to include both turbulence and rotation in the formula for the lincwidth in such narrow lined dwarfs? In this section we look at the effects of ignoring rotation entircly. 


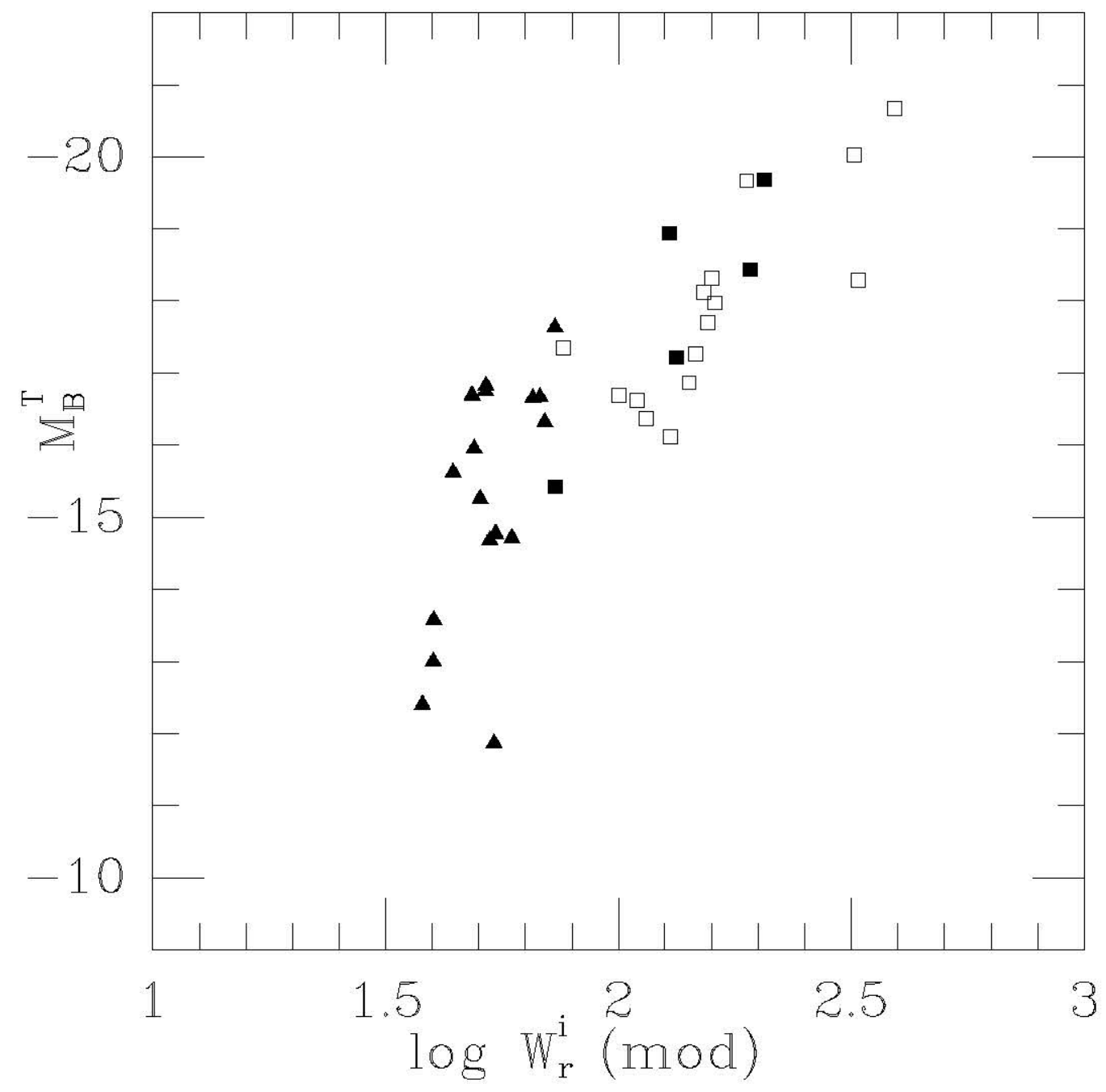

Figure 84: Modified Tully-Fisher Relation. Modified linewidth calculated from equation (4.17) (See texl). Open squares are from Pierce \& Tully (1992); Filled squares are galaxies from this sample that have Double IIormed III profiles; Filled triangles are Gaussian profiled galaxies. 

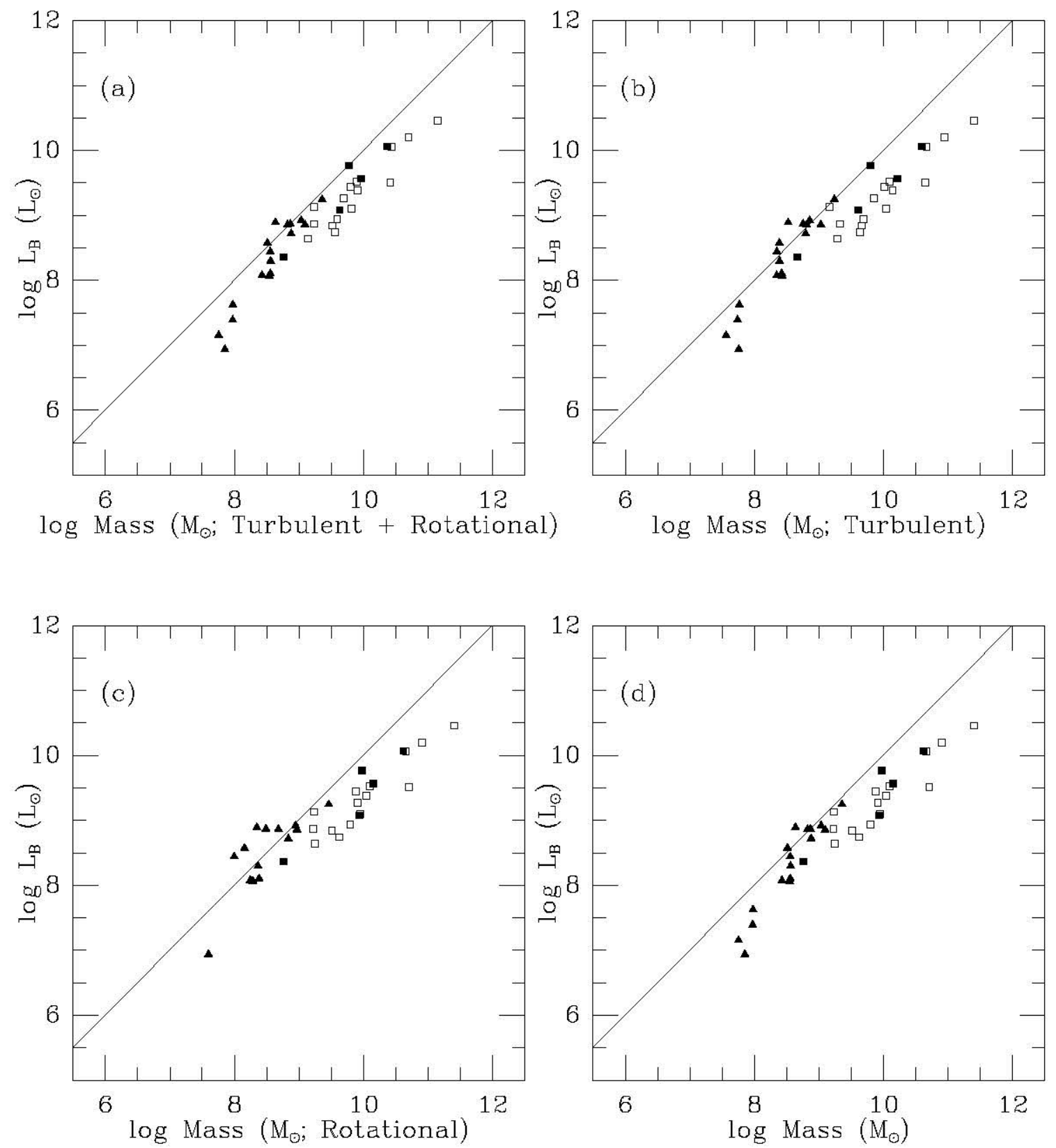

Figure 85: Virial Mass/Luminosity Diagrams. Symbols are as in Figure 84. (a) Mass calculated from equation 4.16 (See text). (b) Mass calculated from (4.18) assuming that the linewidth arises from turbulence alone. (c) Mass calculated from (4.14) assuming that the Tully-Fouqué formulation for the linewidth is correct (constant turbulence of $38 \mathrm{~km} \mathrm{~s}{ }^{-1}$ ). (d) Mass as in (a) for Gaussian profiled galaxies and as in (c) lor Double IIormed profiles. In each panel, the solid line represents $M / L=1$. 
If the Gaussian HI linewidth is assumed to be solely due to turbulent motions in the galaxy, then this width can be divided into two different components (Lo et al. 1993): dispersion within a cloud $\left(\left\langle\sigma_{v}\right\rangle\right)$, due to the temperature of the cloud; and dispersion between clouds $\left(\sigma_{c-c}\right)$, duc to random motions of individual clouds in the potential well of the galaxy. The observed single-dish velocity dispersion $\left(\sigma_{T}=W_{20} /(3.5882)\right)$ is then just the quadratic sum of the two components.

VLA observations of nine dwarf galaxies by Lo et al. find a range for $\left\langle\sigma_{v}\right\rangle$ (averaged over the entire galaxy) of between 4.0 to $8.5 \mathrm{~km} \mathrm{~s}^{-1}$, with an average of $5.1 \mathrm{~km} \mathrm{~s}^{-1}$. Adopting this valuc, and setting $V_{\text {rot }}=0$ in cquation (1.11), we have

$$
M_{v i r}^{t u r b}=2.33 \times 10^{5} r_{\text {vir }} 3\left[\left(W_{20} / 3.5882\right)^{2}-(5.4)^{2}\right] .
$$

Ihe virial mass from turbulenes alone is plotted versus the luminosity in Figure $85(\mathrm{~b})$ and, for comparison, in Figure $85(\mathrm{c})$ we plot the virial mass due to rotation alone (equation 4.14).

The linewidth for spiral galaxies, which are dominated by rotation (double horned), are described adequately by the Tully-Fouqué approximalion. In lact, it is misleading to apply the modified linewidth developed in 81.1 to spirals, because the observed axial ratio for a spiral is not an indication of the intrinsic axial ratio, and henec the velocity anisotropy (Staveley-Smith et al. 1992); but rather is merely an indication of the inclination of a disk which is assumed to be intrinsically circular (Holmberg 1958; Bottinelli el al. 1983). Therefore, in Figure 85(d) we plot the $M_{v i r}^{\text {turbtrot }}$ from equation (1.16) for the galaxics which exhibit Gaussian profiles (turbulenes dominated)

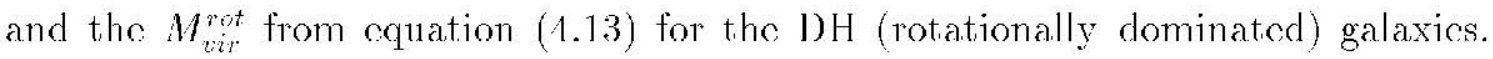
Again, in this sample, it is clear that there is a difference in the $M / L$ for the two classes ol galaxies. 


\subsection{Is the Effect Real?}

In order to see il the dichotomy between turbulent- and rotationally-supported galaxies is observed to occur for all galaxies in the manner we have seen above, wo expanded the sample by including data from the literature. It is necessary for each additional galaxy to have an accurate: absolute magnitude (which requires an accurate distance): axial ratio (in order to calculate either the inclination or $\beta$, the velocity anisotropy); and III linewidth, as well as a III profile with high signal-tonoise in order to classify it as cither Gaussian or Double Horned.

In Figure 86, we plot the virial mass versus the luminosity for galaxics from our own sample as well as additional points from the literature. As in Figure 85(d),

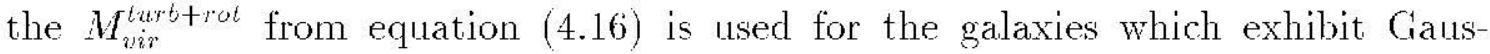
sian profiles (turbulence-dominated) and the $\boldsymbol{M}_{v i r}^{\text {rot }}$ from equation (4.13) for the DII (rotationally-dominated) galaxies. The additional points are from Staveley-Sinith, Davics, \& Kinman (1992), from their obscrvations of dwarf and ISB galaxics: McGaugh (1992) (also McGaugh \& Bothun 1994), from his observations of mostly LSB galaxies: Lo, Sargent, \& Young (1993), from their VLA observations of extremely Caint dwarl galaxies; Pierce \& Tully (1992), the Tully-Fisher calibrator galaxies from the previous figures; Romamishin, Strom, \& Strom (1983), from their sample of LSB spirals; 'Tully (1988) (NBG); from which we sclected all galaxics with $M_{B}^{T}>-16$ for which there was an accurate $\Delta V_{20}$; and Carignan, Demers, \& Côté (1991), for data on the Phoenix dwarf galaxy, which appears to be an intermediate type between dwarf spheroidal and dwarl irregular (see also van de Rydt, Demers, \& Kunkel 1991).

The solid line represents the relation $M / L=1$, and any locus of constant mass to light ratio must be parallel to this line. 'l'he two dotted lines are not fits to the data. 


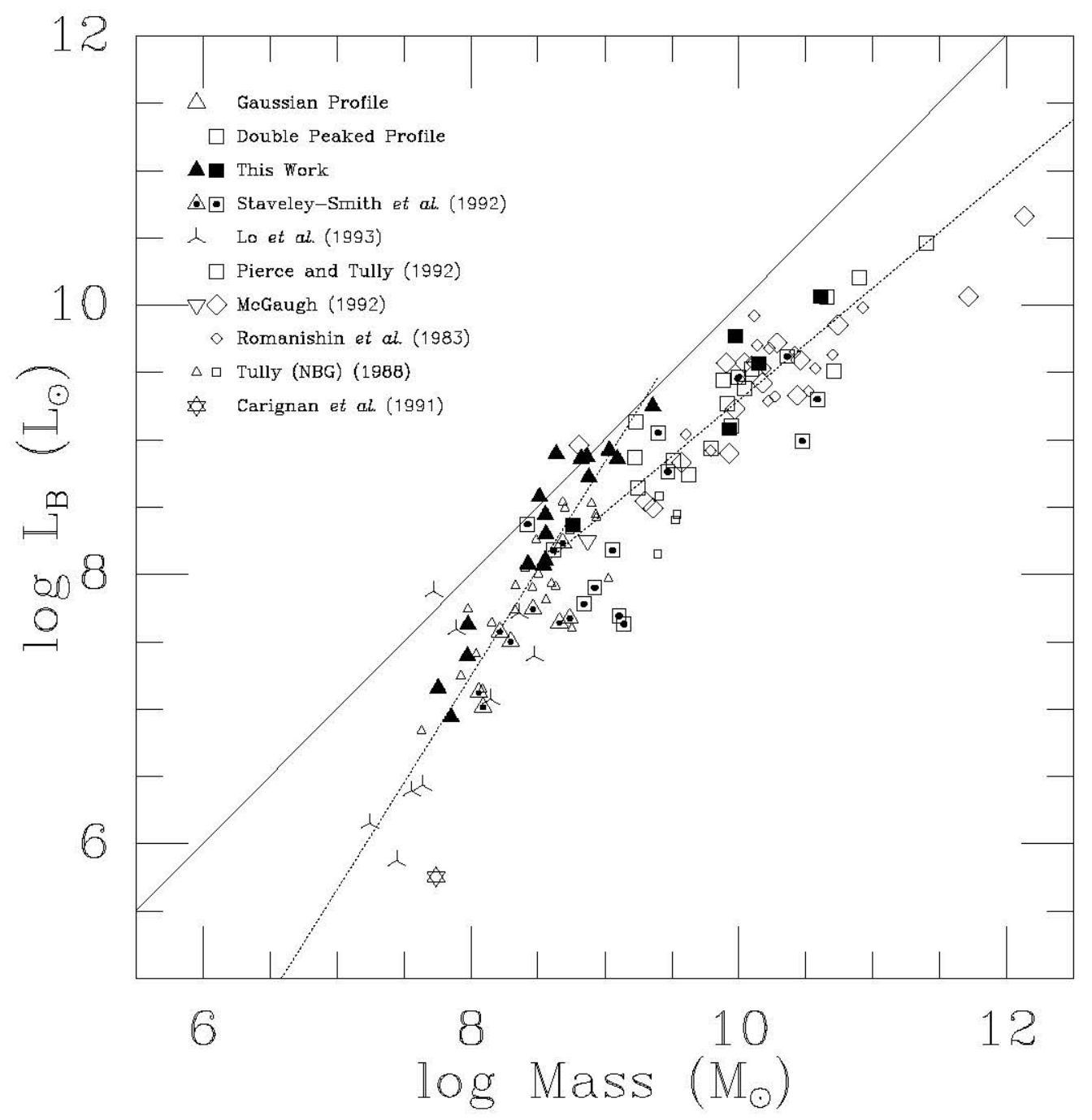

Figure 86: Virial Mass/Luminosity Diagram. Same as Figure 85(d) with additional points from the literature. The solid line is the line for $M / L=1$. The wo dotted lines are $M / L \propto L^{-0.37}$ (for the Gaussian profiles; see 84.8 ) and $M / L \propto L^{0.2}$ (for the rotating galaxies; see $\$ 4.9)$. They do not indicate fits to the data. 
The dotted line through the Gaussian dwarfs is the relation $M / L \propto L^{-0.37}$, with the zeropoint adjusted to fit the data. This relation arises from the theoretical model of Dekel \& Silk (1986) for supernovae driven mass-loss in dwarl galaxies embedded in a massive dark halo (sec also Blumenthal et al. 1981). In this scenario, the supernovac drive most of the ISM out of the galaxy, which remains bound, due to the dark halo. This model was advanced for the formation of dwarf spheroidals, but Dekel \& Silk speculate that incomplete mass loss may result in the formation of a dwarf irregular. This relation, which is seen to represent a good fit to the data, will be discussed further in 81.8 .

The dotted line through the "normal" galaxies is the relation $M / L \times L^{0.2}$, which is implied by the Fundamental Plane relations between velocity dispersion, surface brightness, and effective radius for elliptical galaxies (Dressler el al. 1987; Ferguson \& Binggeli 1994). The relation also appears to represent a good fit to the spiral data, and is bricfly discussed in 84.9 . The transition occurs at $\log L_{B} / L_{C} \sim 8.3$ or $\log M / M_{0} \sim 8.6$, which is discussed in 81.8 .1 .

In Figure 87 the Gaussian dwarfs are plotted alone. A formal least-squares fit to the points yields a relation of $M / L \propto L^{-0.30 \pm 0.16}$ (shown as the dashed line) with a dispersion about the fit of 0.94 mag. Similarly, in Figure 88 we have plotted only the DII galaxies. $\Lambda$ least-squares fit yields $M / L \propto L^{0.25 \pm 0.37}$ (the dashed line) with a dispersion of 0.83 magnitudes.

I'he actual relation indicated by the dotted line in Figure 87 is given by $\log L_{B}=$ $1.435 \log M-4.23$ in solar units. The luminosity of these Gaussian dwarfs can therefore be calculated from the mass from equation (4.16), which depends on the observed line width and the axial ratio. The results of the fits for the data in all the 


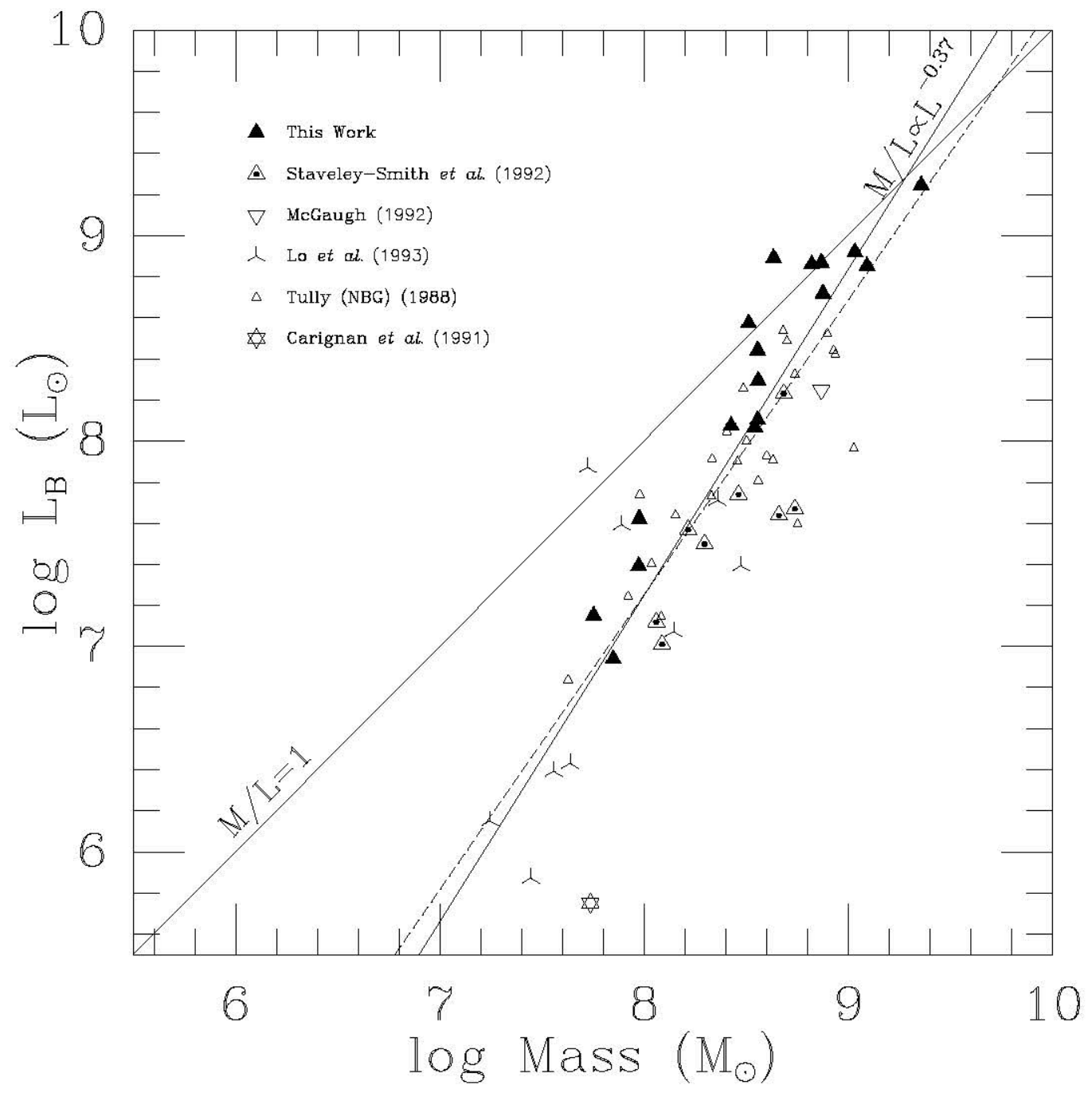

Figure 87: Virial Mass/Luminosity Diagram for Gaussian Profile Galaxies. The solid lines represent the relations $M / L=1$ and $M / L \propto L^{-0.37}$ (Dekel \& Silk 1986; see $\$ 4.8)$. The dashed line is the least-squares fit to the data. 


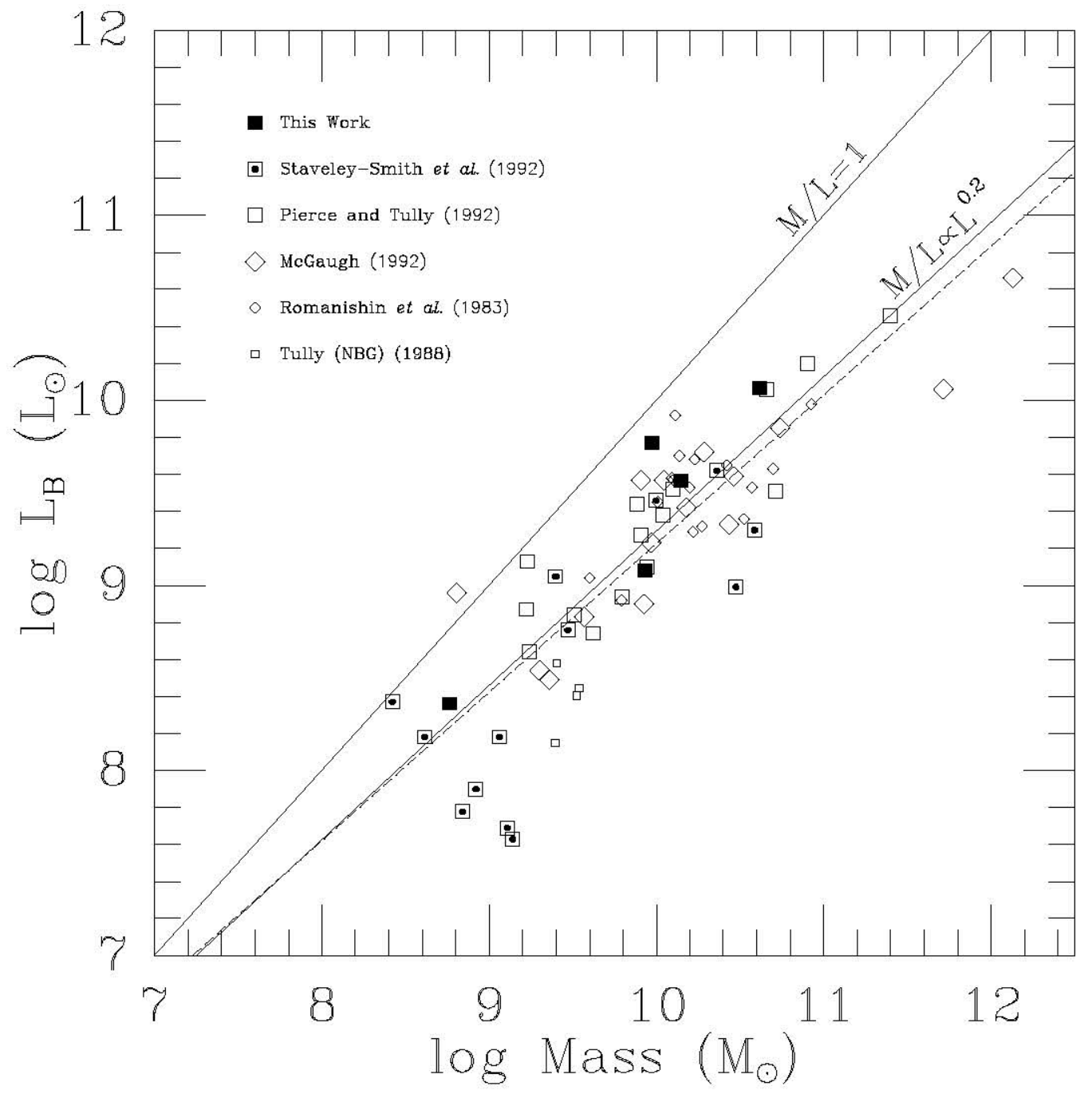

Figure 88: Virial Mass/Luminosity Diagram for DII Sample. The solid lines represent the relations $M / L=1$ and $M / L \propto L^{0.2}$. The dashed line is the least-squares fit to the data. 
figures are listed in Table 9 (\$4.6.1).

In Figure 89, we replot the points from Figure 88, after removing the extreme low surface brightness galaxies from the sample (the data of McGaugh 1992; and Staveley-Smith el al. 1992). What remains are normal spirals (from Pierce \& Tully 1992; Romanishin et al. 1982; and from this sample), usually used to define the Tully-Fisher relation in the first place. Also shown are the LSB spirals from Romanishin, Strom, \& Strom (1982); these are not galaxies of extremely low surface brightness in contrast to the McGaugh sample. The correlation is much improved, and the rolation for elliptical galaxics discussed in $\$ 4.9$, from I)ressler ot al. (1987) is a good fit to the data. 'The formal least squares fit leads to a relation of $M / L \propto \propto$ $L^{0.35 \pm 0.49}$ (the dashed line) with a dispersion of 0.57 magnitudes. For comparison, the intermal rms dispersion for the blue Tully-Fisher relations is on the order of 0.35 mag (Jacoby el al. 1992), the larger dispersion can be attributed to the fact that a sample specifically selected for use in determination of the 'lully-Fisher relation has strict eriteria for inclination, and the distanee is determined independently (and is not simply determined using a redshift with virgocentric correction)

The inclusion of the LSB galaxies would be expected to worsen the fit (as is seen in Figure 88), since the $M / L$ lor these giant disk galaxies, with a very different history of star formation, must be different from that for normal spirals.

\subsubsection{The relation for the $I$-band}

Although the number of suitable galaxies for which $I$-band data is available is small when compared to the data available in the $B$-band, it is desirable to at least check the relation in the longest wavelength available. If the effects $\sec$ in $B$ are 


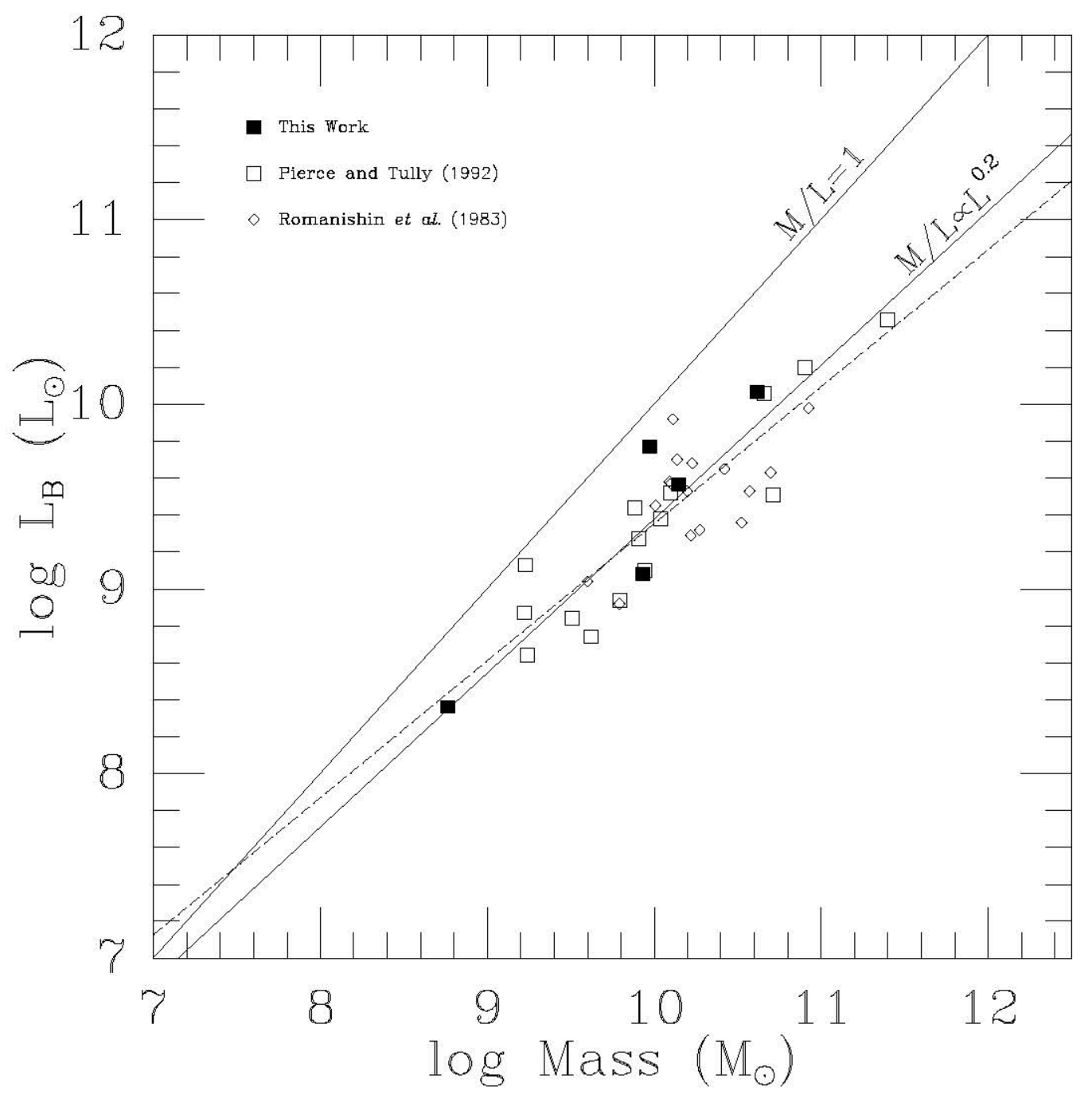

Figure 89: Virial Mass/Luminosity Diagram for DH sample (LSBs excluded). Same as Figure 88, with the LSB galaxies from McGaugh (1992) and Staveley-Smith ef al. (1992) excluded. The dashed line is the least-squares fit to the points in the figure. The close fit of the spiral data to the $M / L \propto L^{0.2}$ relation found for ellipticals (Dressler et al.) indicates that the formative processes are similar. 
purely due to internal extinction corrections, we should be able to discern this from examining the $I$ relation, for which this would be significantly smaller. This was what originally prompted us to obtain data in two widely separated bands. Because the effect seen in Figure 87 is such that it could be (and has been) attributed to increased scatter at the faint end (in Figure 86, for example), we have thus far concentrated on the $B$ relation, for which there was a substantial amount of reliable data available (especially Staveley-Smith el al. 1992; for the luminosity range of interest). In this section we plot the $I$ data and compare the results with the $B$ relation.

In Figure 90 we plot the the Ganssian (a) and 1$) \mathrm{H}$ (b) galaxies for which there is I data (again we exclude the ISSB galaxies of McGaugh in (b)). 'The variation of $M / L$ with $L$ for the Gaussian sample is still pronounced, and appears to have the same slope as seen in Figure 87 for the $B$ data. IIowever the DII galaxies appear to have a nearly constant $M / L$ when observed in $I$. This tendency for the slope of the relation to stecpen to nearly 10 when observing in longer wavelengths is well documented (Aaronson, Huchra, \& Mould 1979; Jacoby ot al. 1992). It is most likely due to both metallicity effects and the larger (and therefore less accurate) correction for internal extinction in $B$ (see \$4.9). The formal least-squares fit to the points in (a) yields an exponent of $-0.26 \pm 0.25$ (shown as a dashed line; compare to the solid line with an exponent of -0.37 ) with a dispersion of 0.90 mag. 'T'he fit to (b) gives an exponent of about $0.02 \pm 0.34$ with a dispersion of 0.75 magnitudes (the dashed line). These fits are summarized in Table 9. In the table the sample, corresponding figure number, and band in which the observations were made are listed. This is followed by the slope (a) and zeropoint (b) (with errors) of the relation $\log L=a \log M+b$ in solar units. The exponent $\alpha$ of the luminosity dependence of $M / L\left(\alpha L^{\alpha}\right)$ is listed next, along with its error. Finally the r.m.s. of the fit in dex is listed along with the 

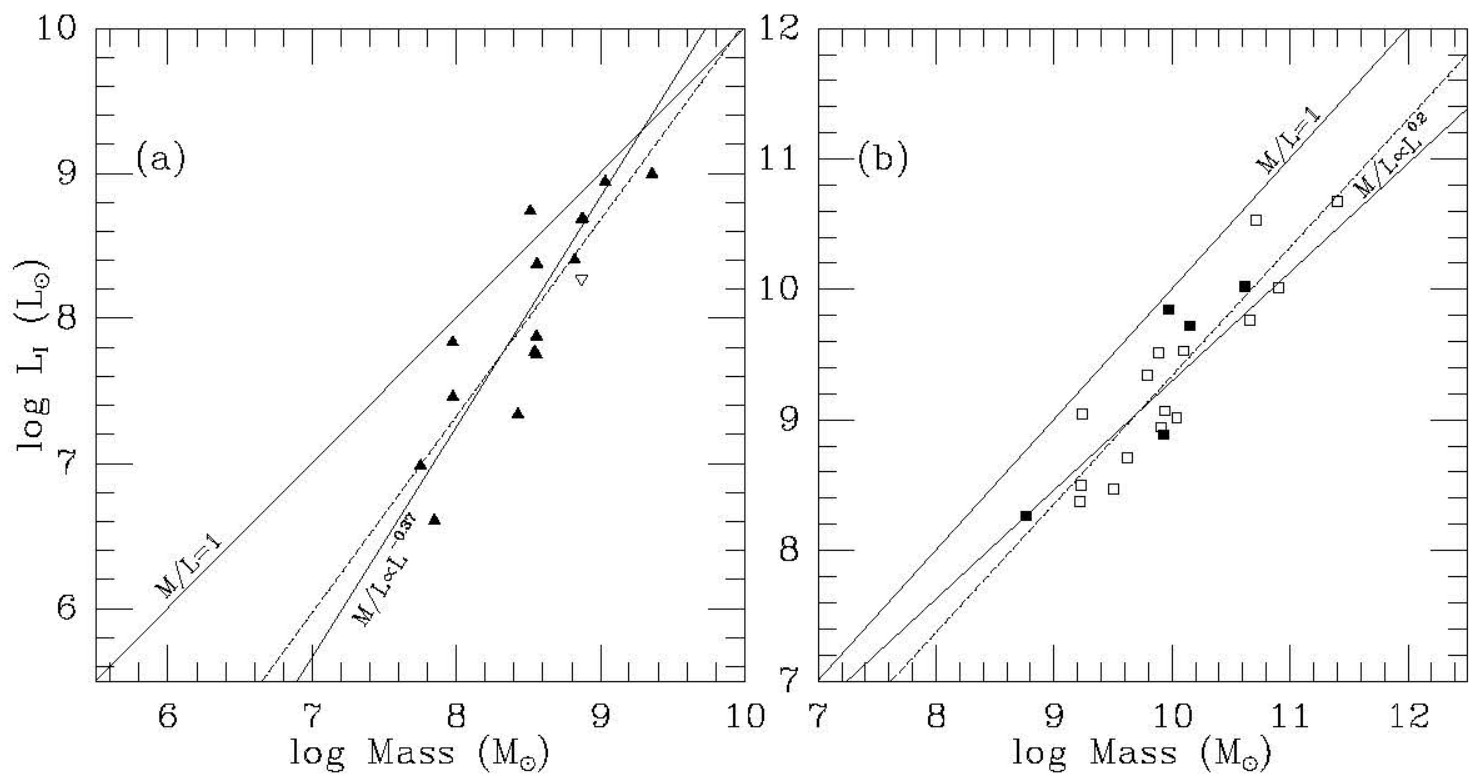

Figure 90: I-band Virial Mass/Juminosity I)iagrams. (a) is the same is Figure 87, for the I data (our (Gaussian sample, and one point from McGangh). 'l'he points still scem to follow the $M / L \times L^{-0.37}$ relation from 1)ckel \& Silk (sce 81.8). In (b) the relation is plotted for the I data with I)H profiles (I.SB galaxies excluded, as in Figure 89). The data fall along a relation with a stecper slope than in Figure 89, which would be expected if the $M / L \propto L^{0.2}$ trend in Figure 89 is due to metallicity effects. The trend in the $I$-band is one of nearly constant $M / L$. See $\$ 4.9$. 
dispersion in magnitudes $\left(\sigma_{m a g}\right)$.

The important point in the context of this work is that the slope for the Gaussian sample is not dependent on the wavelength of the observation. This again points to the lact that this is not an observational effect, but is an intrinsic difference present between the Gaussian (dwarf) sample and the I)H (spiral) sample.

Table 9:

Mass/Luminosity Fitting Results for Samples

\begin{tabular}{llcccrrrr}
\hline \hline Sample & Figure & Band & $\#$ & $\mathrm{a}$ & \multicolumn{1}{c}{$\mathrm{b}$} & \multicolumn{1}{c}{$\alpha$} & r.m.s. & $\sigma_{\text {mag }}$ \\
\hline Gaussian & 87 & $B$ & 60 & $1.44 \pm 0.33$ & $-4.23 \pm 0.96$ & $-0.30 \pm 0.16$ & 0.377 & 0.94 \\
DH (spirals) & 88 & $B$ & 68 & $0.80 \pm 0.24$ & $1.20 \pm 0.76$ & $0.25 \pm 0.37$ & 0.330 & 0.83 \\
"good" DH & 89 & $B$ & 36 & $0.74 \pm 0.27$ & $1.93 \pm 0.85$ & $0.35 \pm 0.49$ & 0.227 & 0.57 \\
(raussian & $90 \mathrm{a}$ & 1 & 16 & $1.36 \pm 0.46$ & $-3.52 \pm 1.35$ & $-0.26 \pm 0.25$ & 0.359 & 0.90 \\
DII & $90 \mathrm{~b}$ & $I$ & 20 & $0.98 \pm 0.33$ & $-0.19 \pm 1.05$ & $0.02 \pm 0.31$ & 0.300 & 0.75 \\
\hline \hline
\end{tabular}

\subsection{Surface Brightness and Color: Distance In- dependent Tests}

In examining the physical meaning of the IR Tully-Fisher relation (Aaronson tt al. 1982), Burstein $(1982,1988)$ found that the observed relation did not imply a constant $M / L$, since the $M / L$, was found to depend on the luminosity. In order to investigate the relation in a distance independent manner, he formed a hybrid surface brightness from the $H$-band aperture magnitudes.

We will adopt this surface brightness for the DH sample of galaxies:

$$
S B^{i}=B_{T}^{0}+5 \log R_{\text {tir }}+2.5 \log (b / a)
$$


where the total apparent magnitude, $B_{I}^{0}$, is from Table 6 or the literature; $R_{\text {vir }}$ is the virial radius used in equation (4.13) but expressed in arcseconds, and $b / a$ is the axial ratio. For the Gaussian sample, we use the same expression, without the correction for inclination, as discussed in $\$ 3.1 .1$,

$$
S B=B_{T}^{0}+5 \log R_{v i r}
$$

In Figure 91, we plot the line width versus the surface brightness. In (a) the linewidth with corrections from Tully \& Fouqué (1985) (equation 3.18) is plotted lor all the points, while in (b) the modified line width (equation 1.17) is used for the Gaussian dwarfs. While there is more scatter, it is clear that the diagram is showing the same effect seen in Figure 85 (c) and (d). As pointed out by Pierce \& Tully (1992), this surface brightness relation (Figure 91) should be insensitive to effects of star formation and extinction in the sample, but sensitive to changes in the III propertics. In neither case do the Gaussian dwarfs fall on the same relation as the normal (I)H) spirals. The significantly smaller dispersion for the relationship seen in Figure 91 (b) gives us confidence that our procedure for correcting the linewidth for turbulence is correct. Again, this relation seems to indicate that there is not only a tight relationship between the mass and light within the Gaussian dwarl sample, but also that it is distinct from the relationship found for the sample of spirals. Again, the LSB points in the DH sample are excluded (see above), as well as the LSB spirals from Romanishin et al. and McGaugh.

The color/linewidth relation is shown in Figure 92, with the linewidths calculated in the same way as in the previous figure. $\Lambda$ ll of the data for which $B-I$ colors exist are plotted. 'This includes most of the galaxics in this sample, as well as the ISB galaxics of McGaugh and the normal spirals from Picres \& Tully. Picrec \&. 


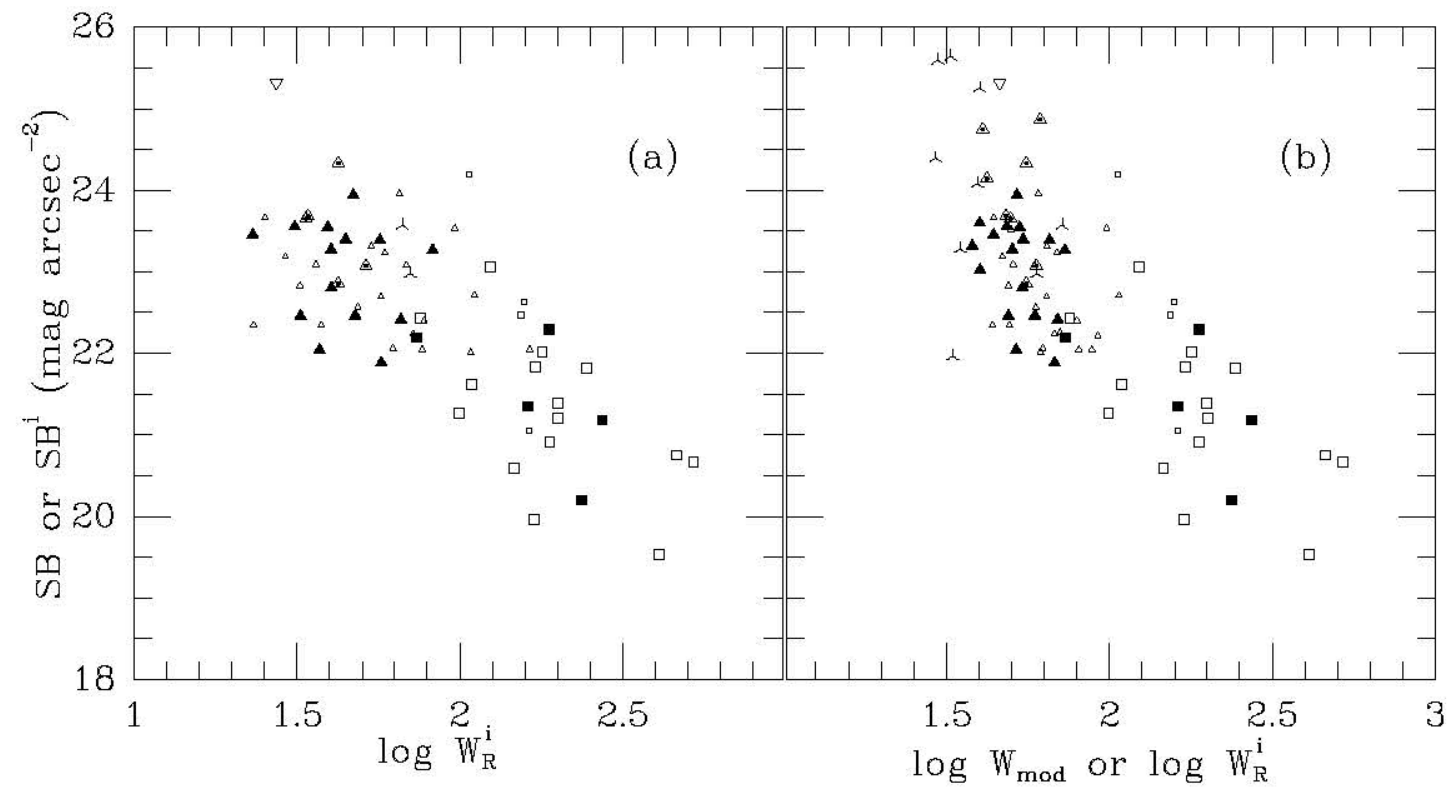

Figure 91: Surface-Brightness/Linewidth relation. (a) is a plot of the observed linewidth, corrected as in equation (3.18) versus the $B$ surface brightness either from equation (4.19) for the DH sample or from equation (4.20) for the Gaussian sample. In (b), the modified form of the linewidth (equation 4.17) is used for the Gaussian sample. All symbols are the same as in Figure 86. Compare the lack of correlation for the Gaussian dwarls in (a) with Figures 81(a) and 85 (c). The relation seen in (b) for the Gaussian dwarls, different from that for the DII sample, should be compared to Figures 84 and $85(\mathrm{~d})$. The presence of the effect in the surface-brightness/linewidth indicates that the correlation seen in the earlier figures is nol a distance eflect (Burstein 1982). The the different behaviours of the Gaussian sample and the $\mathrm{l}$ H sample in cither figure suggests that the dichotomy discussed in 81.8 is real. 
Tully note that this diagram is sensitive to recent differences in star formation. The Pierce \& Tully spirals (open squares) form a continuum along with the DII galaxies from our sample, while the LSB disks lie to the red side of this, implying less star formation, which is obviously the case in these "crouching giants". In contrast to this, the Gaussian sample is distributed over a large range in color, with hardly any spread in the linewidth.

The color/linewidth relation is also sensitive to the internal extinction, and since we have chosen not to apply any such correction to our sample ( $\$ 3.1 .1)$, we should perhaps be concerned that the scatter in Figure 92 is largely due to uncorrected internal extinction. However, the range in color observed $(\sim 2.5 \mathrm{mag})$ is much greater than would be expected from any model for internal extinction. In addition, we have already seen (\$4.3.2, Figure 83(a)) that there is no correlation of the Tully-Fisher residual with inclination.

\subsection{Evidence for Mass-Loss within a Dominant Dark Halo}

\subsubsection{Theoretical Basis}

The precipitous drop in the luminosity with mass below an apparent threshold of $\sim 10^{8.5} M_{\odot}$, which is close to the minimum mass for a stable, rotating disk (Toomre 1964; Fall \& Efstathiou 1980; Ashman 1990), can be understood naturally within the framework of supernovae driven mass-loss within a dark halo, according to Dekel \& Silk. Because the energy from the supernovac will drive much of the ISM out of the 


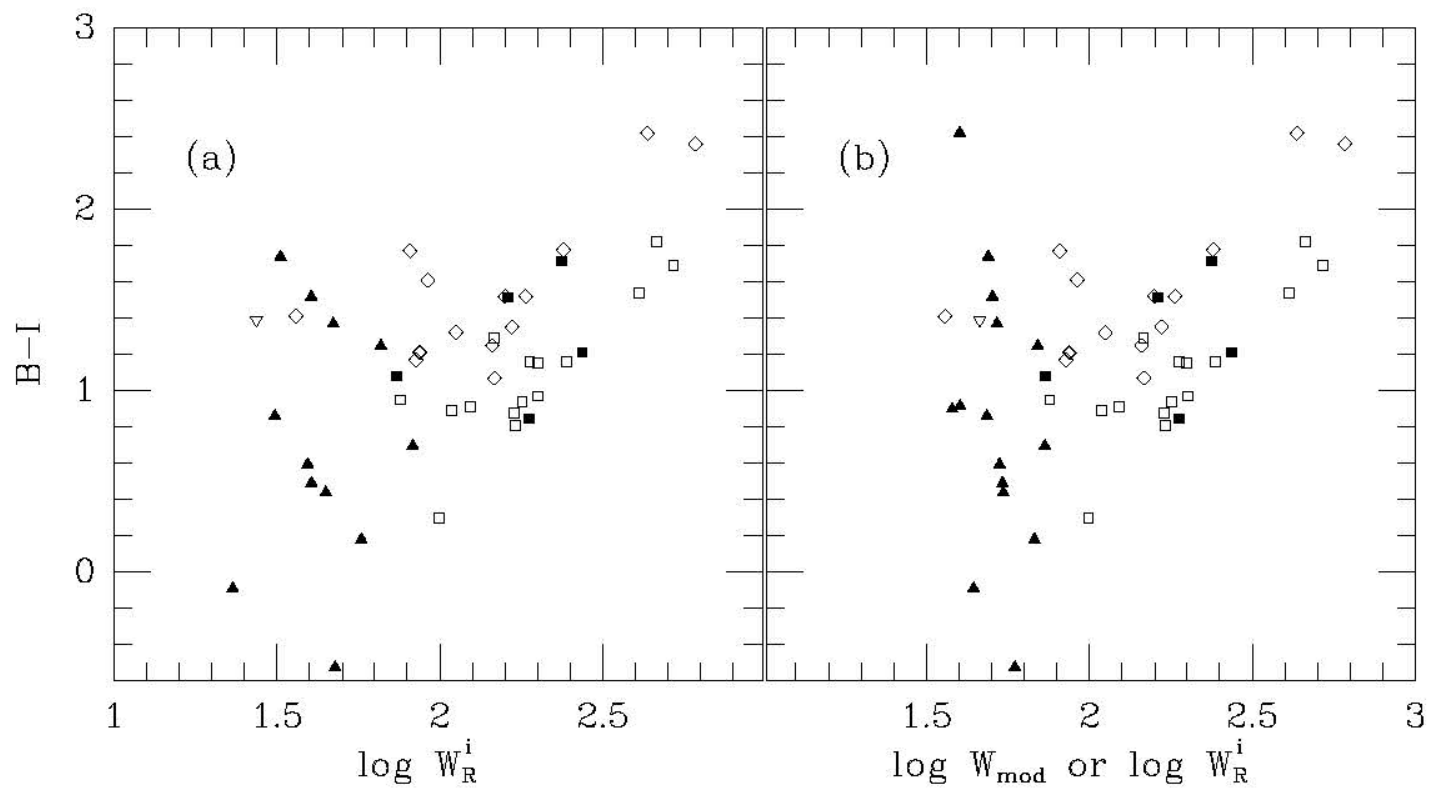

Figure 92: Color/Linewidth relation. (a) is a plot of the observed linewidth, corrected as in equation (3.18) versus the $B-I$ color for all the galaxies with $I$ data. In (b), the modified form of the linewidth (equation 4.17) is used for the Gaussian sample. All symbols are the same as in Figure 86. Again, the Gaussian dwarls do not follow the relation seen for the DII sample, with the DII galaxies getting bluer as the linewidth gets smaller (also seen in Pierce \& Tully 1992). The dispersion increases dramatically with the Gaussian sample, and the color and linewidth appear uncorrelated, suggesting different star formation histories for the two samples. 
galaxy, if the galaxy's mass (including the dark halo) is low enough, star formation will drop dramatically (in the case of $\mathrm{dIs}$ ) or cease (in the case of dEs, where the entire ISM has been removed). The dark matter halo is crucial in order to keep the dwarf bound after much (or all) of its gas has becn removed.

I'he supernovac driven mass loss scenario also will rid the galaxy preferentially of high metallicity gas (in the form of the supernova ejecta itself) (Vader $1986 ; 1988$ ). This would explain naturally the extremely low-metallicity of dwarfs, while still allowing more than one generation of star formation to have occurred. It has long becn realized that simple chemical cvolution models of BCI)s which do not take into account mass loss cannot reproduce the heavy element abundances and gas mass fractions observed (Matteucci \& Chiosi 1983).

This model is also attractive because of its possible explanation of the existence of laint blue galaxies in deep CCD frames (Tyson 1988). Babul \& Rees (1992) propose that these objects are dwarl ellipticals undergoing there first burst of star formation, which drives away the remaining ISM in this scenario. 'l'hey identify present-day dwarf ellipticals as the surviving members of this population, preserved in high pressure environments, while those formed in low pressure regions would fade away. It is not clear if this can fully explain the discrepancy between the number of faint blue galaxies inlerred to exist from observations and the local galaxy luminosity function.

Dekel \& Silk determine the critical velocity, $V_{\text {erit }}$, below which substantial gas loss is possible. This velocity is dependent on the density and temperature of the interstellar medium, given reasonable assumptions for the amount of energy generated by supernovae. They find $V_{\text {crit }}$ to be only very weakly dependent on the gas 
number density, with a value $\sim 100 \mathrm{~km} \mathrm{~s}^{-1}$.

'l'herefore, in this scenario, dwarfs are simply galaxics with virial velocitics below

about $100 \mathrm{~km} \mathrm{~s}^{-1}$ which have experienced substantial (or complete, in the casc of dEs) mass loss after a primary burst of star formation. This velocity corresponds to a mass of $\sim 10^{8.5}-10^{9.5} M_{\odot}$. The range is masses is due to the fact that the critical mass depends not only on $V_{\text {crit }}$ but also the luminosity of the galaxy (see Figure 6 of I) \& \& Silk).

\subsubsection{Observational Evidence}

Although this model was the theoretically favored explanation for the striking differences between dwarl and massive galaxies (c.. Silk. Wyse, \& Shields 1987; De Young \&. Gallagher 1990: Sandage \&. Hoffman 1991: Wyse 1993; and 1)e Young \& Heckman 1994), little observational evidence existed for such outflows of supernovae driven gas until Meurer et al. (1992) found dramatic evidence for an expanding superbubble of ionized gas in the BCD, or "post-starburst" galaxy, NGC 1705. Subsequent work by IIunter, IIawley, \& Gallagher (1993) and Marlowe el al. (1994) provide evidence that this phenomenon is occurring in a number of low luminosity starburst galaxies. In addition, Papaderos et al. (1994) find evidence from ROSAT data for hot gas outflow in the BCD VII Zw 403 and Thuan et al. (1994) find very broad line components in II $\alpha$ profiles in a number of BCDs. The III holes observed by Puche ct al. (1992) in Holmberg II also support this picture.

Much effort has been devoted to determining $M / L$ for the local df galaxics (c.g. Aaronson \& Olszewski 1987; Bender \& Nicto 1990; Bender, Paquet, \& Nicto 1991; Carter \& Sadler 1990). The results seem to support the Dekel \& Silk model 
$\left(M / L \propto L^{-0.37}\right)$, although the difficulties in measuring radial velocities for faint, weak lined stars are considerable (Peterson \& Caldwell 1993; Ferguson \& Binggeli 1994).

Because the model was mainly conceived as a way of explaining the observed propertics of gas depleted dis, with the inclusion of dIs nearly as an afterthought, little attention has been paid to dIs in the light of this model. Pierce (1991) does note that the $M / L$ for disk galaxies below $\log L_{I} / L_{\odot} \sim 9.8$ appears to change abruptly (compared to a transition seen in Figure 90 at $\log L_{T} / L_{6} \sim 8.5-9.0$ ), and suggests the 1)ckel \& Silk scenario as a possible explanation. However, it scems unlikely that the I)ckel \& Silk dwarfs could remain disk systems. 'The quantity and global nature of the predicted mass-loss, while not completely disrupting the galaxy due to the dark matter halo, seems likely to disrupt any disk, leaving a chaotic dwarl behind. The lack of dwarl spirals, as well as the high anisotropy of dEs supports this. The disk galaxies in Pierce's (1991) sample could have undergone at best a very mild form of mass loss in the I)ekel \& Silk scenario, more dramatic effects would only be observable in less massive, "true" dwarfs, where the escape velocity is sufficiently low to allow substantial mass loss.

At this point a "critical mass" of accurate CCD data (along with the III data) for Gaussian profiled dwarls has accumulated from various authors, notably StaveleySmith et al. (1992) and those cited by lo et al. (1993) for their extreme sample of VLA dwarfs, as well as the observations presented here. These observations, in conjunction with those of $\mathrm{dEs}$, as well as the observations of outflows in starbursting dwarls (Meurer el al. 1992; Marlowe el al. 1994; Papaderos el al. 1994), finally bring a quantity of evidenec to support I)ekel \& Silk's original suggestion that the formation of both $\mathrm{dF}$ and $\mathrm{dI}$ galaxics could be understood in terms of supernovac 
driven mass-loss in a dominant dark halo.

\subsection{Mass to Light Dependence in Massive Galax- ies: Implications for Spirals from the Tully- Fisher Relation}

We now put our results for low-luminosity systems in the context of more luminous systems. We examine the $M / L$ versus $L$ dependence in massive galaxies, in light of the similarity of our Figure 86 to Figure 1 (for ellipticals) of Ferguson \& Binggeli (1991), not only at the dwarf end, but also in the regime of normal spirals and

ellipticals. It appears from both of these figures that the $M / L$ depends on $L$ in the same way over the same range, which suggests a similarity in the formative processes for ellipticals and spirals, as well as dEs and dIs (as discussed above).

The relation between the central velocity dispersion $\left(\sigma_{0}\right)$, the effective surface brightness $\left(l_{e}\right)$, and effective radius $\left(r_{e}\right)$ for clliptical galaxics, is well described by the scaling law which restricts ellipticals to the Fundamental Plane of this parameter space (Dressler et al. 1987; Djorgovski \& Davis 1987):

$$
r_{e} \propto \sigma_{0}{ }^{A} l_{e}^{B}
$$

where $A \sim 1.4$ and $B \sim-0.9$ (Kormendy \& Djorgovski 1989; Bender, Burstein, \&. Faber 1992). Dressler et al. show that in conjunction with the virial theorem, the above scaling relation implics a relation approximately of the form $M / L \propto$ $L^{0.2}$ (Ferguson \& Binggeli 1994). They also note that much of this dependence on luminosity is due to the bolometric correction, since the brighter ellipticals are 
systematically redder, due to metallicity effects (Faber \& Jackson 1976; Smith \& Tinsley 1976)). (For discussions on the deviation of the dEs from the Fundamental Plane, see Wirth \& Gallagher 1984; Kormendy 1985, 1987; Nieto el al. 1990)

If the $M / L$ for spirals was also taken to be proportional to $L^{0.2}$, due to metallicity effects (Bothun et al. 1981), or for some other reason, then (from equation 1.8) the slope of the Tully-Fisher Relation is expected to be 7.14 rather than 10 . This assumes that $L \propto R^{2}$, which is at least a reasonable assumption (see Figure 93 in chapter 5). The fact that this slope comes out so close to the observed slope for the $B$-band relation (7.18; Picre \& 'lully 1992) suggests a connection. 'The slightly stecper slope found in the redder relations appears consistent with this idea (Figure $90(\mathrm{~b}))$. It seems likely that the dependence of $M / L$ on $L$ seen for spirals in $B$ is due to the effects of metallicity, with some increased scatter due to the large and uncertain correction for internal extinction.

\subsection{A Distance Indicator for Dwarfs?}

The usefulness of the modified 'T'F' (M'T'F) relation as a distance indicator for dwarf galaxics is discussed in this section. We have seen that a wavelength independent relationship between mass (calculated from the HI linewidth) and luminosity exists for dwarf galaxies with Gaussian HI profiles (Figures 87 and 90a), and that this is well fitted by the Dekel-Silk model $\left(M / L \propto L^{-0.37}\right)$.

This relationship exists despite the uncertain photometry (from various sources) for the Io et al. (1993) sample of extremely faint dwarfs. The uncertainty in the actual distance for many of the dwarfs has also increased the scatter along the $\log l$. 
axis. However, the steep slope of the relation means that the dispersion from the fit will be small even for large changes (errors) in $\log L$.

The standard deviation of the residuals to the fit along the $\log L$ axis in Figure 87 is $0.94 \mathrm{mag}$, compared with 0.57 mag lor the "good" DII galaxy fit shown in Figure 89 and $0.83 \mathrm{mag}$ for the entire $\mathrm{I}) \mathrm{H}$ sample (Figure 88). While the W'I'F' relation secms to be a less accurate distance indicator for dwarfs than the Tully-Fisher relation is for larger galaxies, it would appear that it could prove to be useful.

In order to realize the full potential of this relation accurate distances need to be established for local calibrator dwarls. Because of the unknown star formation histories and lack of other standard candles in these dwarfs, this is a difficult task (sec 83.2 for a discussion and referenees regarding the distance to GR8). Another approach would be to obtain accurate photometry for a large number of dwarfs in the Virgo cluster and establish an accurate slope for the relation. An accurate distance for the Virgo Cluster from other means, such as Cepheid variables (Pierce et al. 1994 ) would also allow the zeropoint to be fixed. I)warfs that are faint enough to fix the low end of the relation would be difficult to discover optically over an area as large as that subtended by the Virgo cluster (Sandage \& Binggeli 1984), so a systematic deep III survey would be necessary, with deep followup CCD photometry (for a discussion of the detection of dwarfs in III, systematically or serendipitously, sce Hoffman, Helow \& Salpeter 1988; Briggs 1990; Weinberg et al. 1991; and Taylor, Brinks, \& Skillman 1993).

At this stage, the MTF relation is most useful as a confirmation of a theoretical model for galaxy formation, and further work should emphasize determining the slope of the relation. In addition, the MTF relation can also be used to check the 
distances of dwarfs determined by other methods, but the accuracy of this method as a distance estimator is limited by the uncertainty of the calibration. One potentially uselul sample of dwarls with independently determined distances are those observed by 'T'ikhonov \& Karachentsev (1993) and Karachentsev \& T'ikhonov (1991) for which photometric distances have been determined from the mean apparent magnitude of the 3 brightest stars in the galaxy.

If the MTF should prove to be a reliable distance indicator for dwarfs, the numerous III observations of dwarl's (Fisher \& Tully 1981; Schneider el al. 1990; 1992) could, in conjunction with followup (CO) obscrvations, be used to to examine bulk flow motions (c.g., Jynden Bell et al. 1988). In addition, dwarfs with accurate poculiar velocities can be used as test particles to probe the dark matter content of groups and clusters of galaxies (Zaritsky \& White 1994.) 


\section{Chapter 5}

\section{Global Properties and}

\section{Comparative Structure of Dwarfs}

In this chapter, we compare the various global quantities and derived structural parameters for the galaxies in our sample. By comparing the location of our sample in these various parameter spaces with the location of other types of galaxies, we gain some insight into the relationship between these types.

\subsection{Surface Brightness}

In Figure 93(a), the relationship between the virial radius $\left(R_{v i r}\right)$ and the luminosity is plotted for all the galaxies from this sample and the sources in the literature cited in 34.6. The solid line represents a relation of constant surface brightness. The data show a great deal of scatter, but do follow the relation. In Figure 93(b), only the 


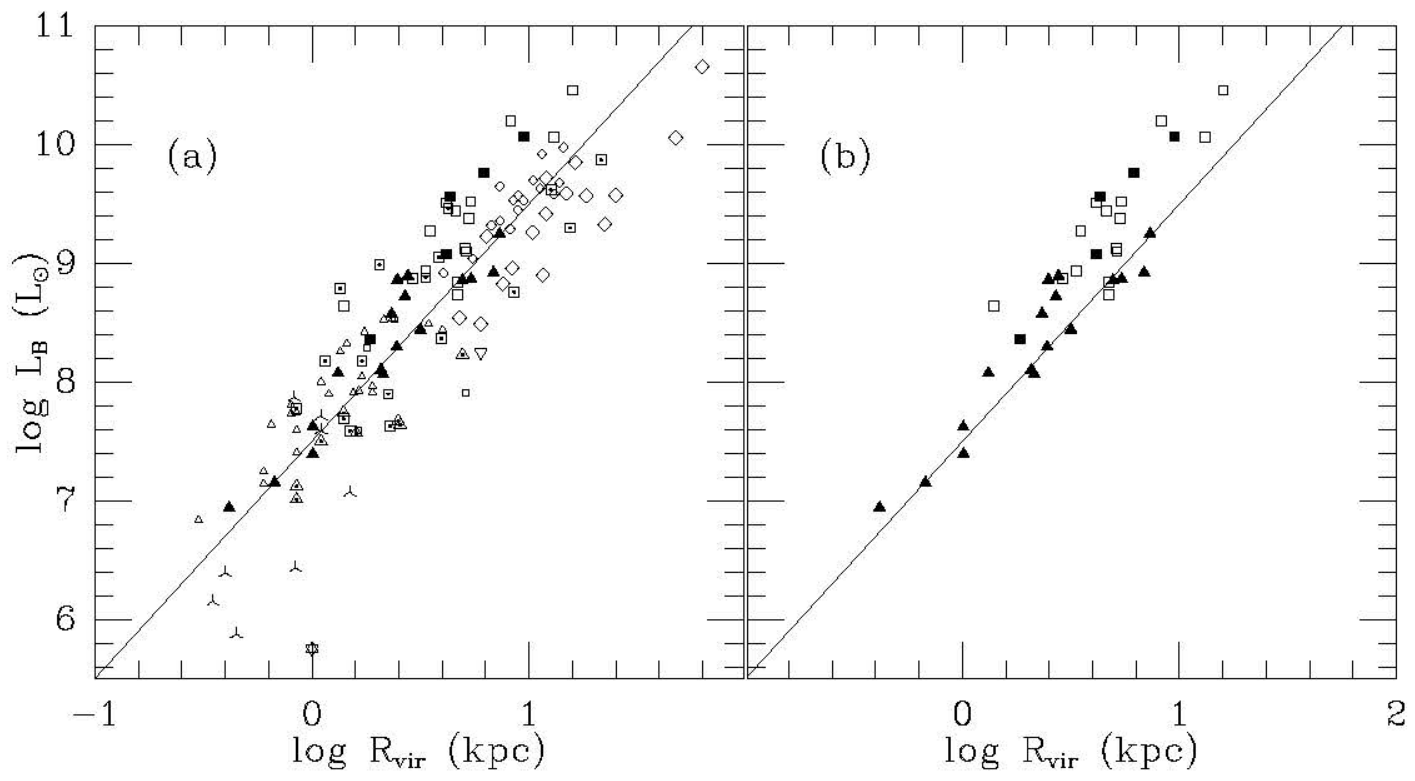

Figure 93: Radius/Luminosity Relation. In (a) the relation is shown for all of the galaxy samples discussed in \$4.6. A relation parallel to the solid line is one of constant surface brightness. Symbols are the same as in Figure 86. In (b) just the Pierce \& Tully (1992) and the galaxies from this sample are plotted. The galaxies in our DII sample have the same surface brightness as the Pierce \& Tully galaxies, while in general the Gaussian dwarls have a lower surlace brightness. Wilhin each sample the assumption of constant surface brightness appears reasonable. 
galaxies from this sample and from the Pierce \& Tully (1992) sample of "normal" spirals are plotted. IIere it is obvious that most of the dwarls follow their own relationship of roughly constant surface brightness $\left(\langle\mu\rangle_{\text {max }} \sim 22.0 \operatorname{mag} \operatorname{arcsec}^{-2}\right)$, slightly fainter than the spirals $\left(\langle\mu\rangle_{\text {max }} \sim 21.0 \mathrm{mag} \operatorname{arcscc}^{-2}\right)$ (sec Figure 94).

Interestingly, the five Gaussians which appear to lic close to the relation for normal spirals ( $\mathrm{CGC} 31, \mathrm{UGC} 63, \mathrm{CGC} 1171, \mathrm{LGC} 3212$, and LGC 7684), appear to have fairly smooth surface brightness profiles. They do not appear to be undergoing a widespread burst of star formation, which would be expected to cause a corresponding increase in the surface brightness. Any star formation secms confined to in or near the nucleus.

The average surface brightness, calculated from cquations (1.19) and $(1.20)$ is plotted in Figure 94. The two extreme points to the lower left are Malin 1-type objects from McGaugh (1992). These are giant disk galaxies with extremely low surface brightness. The dwarl galaxies have a slightly lainter peak surface brightness in this diagram. However they do secm to overlap with both the spiral and ISSB distributions.

The surface brightness of the sky $\left(\mu_{B} \sim 22.7\right.$ mag arcsec $\left.{ }^{-2}\right)$ is indicated by the dashed line in Figure 94. It has long been realized (cf., Disney 1976) that the surlace brightness of the night sky seriously restricts our knowledge of the true galaxy Iuminosity function. Irwin et al. (1990) find no decrease in the number density of ISBs at lower surface brightness levels $\left(\mu_{B} \sim 27.3 \mathrm{mag} \operatorname{arcsec}^{-2}\right)$ 11sing the APM machine to perform an automated search of deep plates covering a large area of the sky. Bothun, Impey, \& Malin (1991) also find evidence for an increase in the slope of the luminosity function at the faint end. McGaugh (1994) postulates that there could 


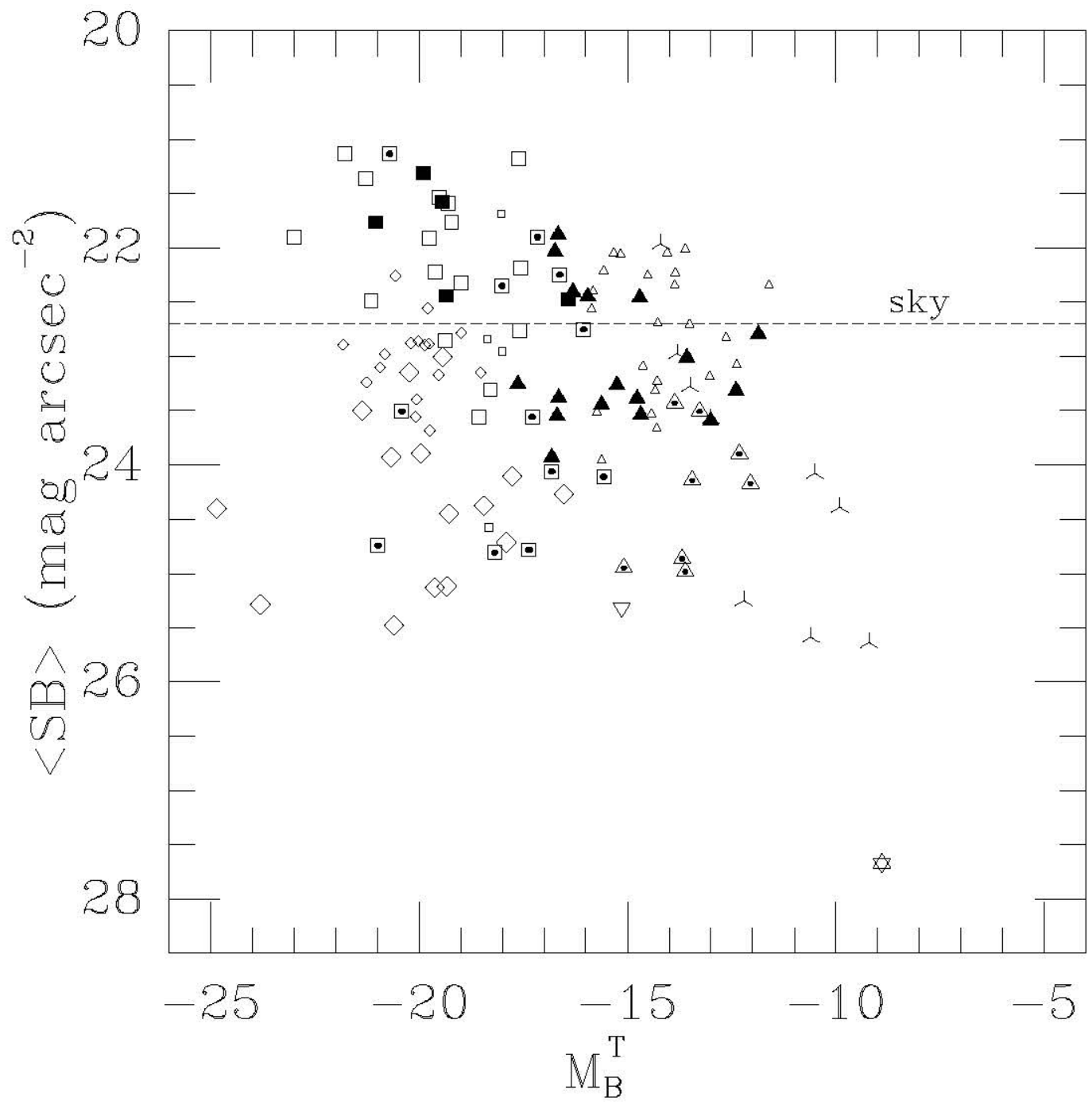

Figure 94: Average Surface Brightness versus Absolute Magnitude. Symbols are the same as Figure 86. "l'he dashed line indicates the sky brightness in $B$ at. Kitt. Peak. The average surface brightness is computed as in \$1.7. The Gaussian and I)H sample cxhibit a similar range in surface brightness, with a slightly lower maximum value observed for dwarfs. This is consistent with the idea of mass-loss in dwarfs (as in the Dekel \& Silk model or any other stripping scenario). The two LSB galaxies at the extreme left are Malin 1-type giant disk galaxies. Phoenix is at the extreme lower right; it was only discovered because of its proximity and hence the ability to detect individual stars. 
be enough LSB "crouching giants" to account for a local population corresponding to the faint blue galaxies at intermediate redshift (Tyson 1988; Broadhurst $t$ l al. 1988). Obviously, we cannot detemine this population through optical surveys. IIowever, cvidence from HI surveys, as well as statistics from "blind" offsets, do not secm to support the existence of a large population of local LSB disk galaxies (cf., Briggs $1990)$

A similar distribution can be seen in the central surface brightness $\left(\mu_{0}^{B}\right)$ versus absolute magnitude diagram (Figure 95), adopted from Kormendy (1987) and Ferguson \& Binggeli (1991). 'This diagram is often used to demonstrate the three distinct classes of stellar systems. To the upper left lic the ellipticals (filled circles) and the bulges of spirals (crosses), making a tight relationship which marks the intersection of the Fundamental Plane (see $\$ 4.9$ ) with this parameter space. The M32-like compact ellipticals also fall on this continumm. Globular clusters populate a distinct section of the diagram.

Spiral disks (plus signs; from Frecman 1970; Boroson 1981; and Bothun ct al. 1985) form a comparatively tight group near $M_{B}^{T} \sim-20$ and $\mu_{0}^{B} \sim 21$. This is partly an observational selection effect due to the surface brightness of the sky, indicated by the dashed line; the more recently discovered LSB galaxies including Malin 1 (Sprayberry et al. 1993), can be seen as part of a continumm extending down from the spirals. The dwarf Irregular and the Iocal Group dwarf Spheroidal (dSph) galaxies appear to fall along an overlapping relation which bends over and extends to extremely faint absolute magnitudes.

There is the potential for much confusion in the literature between the terms dwarl Spheroidal, dwarl Elliptical and compact Ellipticals. Binggeli (1994) and 


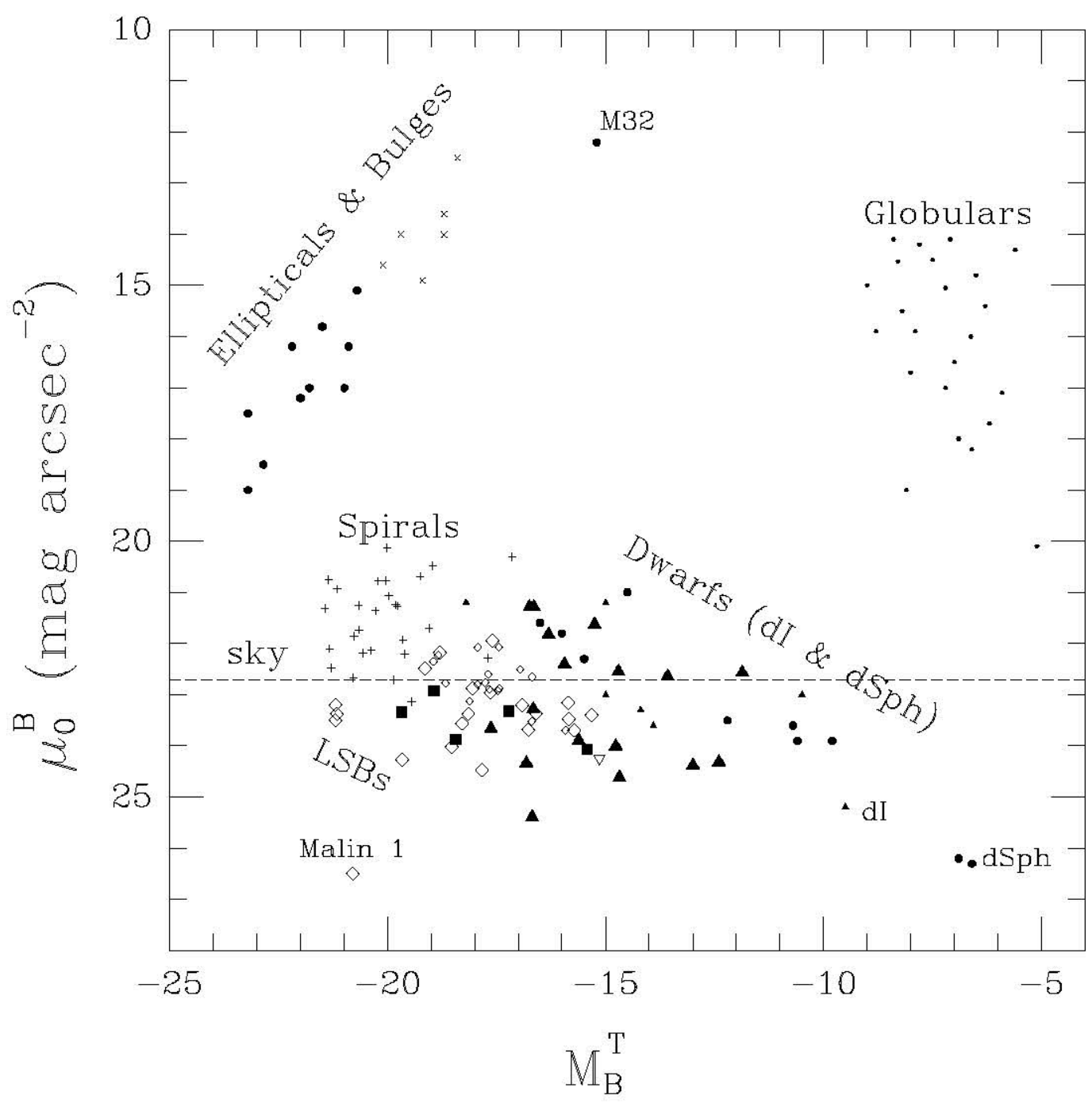

Figure 95: Central Surface Brightness versus $\Lambda$ bsolute Magnitude. Adapted from figures in Kormendy (1987) and Binggeli (1994). The intersection of the Fundamental Plane for ellipticals $(\bullet)$ and bulges $(x)$ is at the upper left. $\Lambda$ separate area of the plane is populated by globular clusters. Spirals (+; and the I)H sample; filled squares) and IsSBs (from McGaugh, large $\diamond$; and from Romanishin et al. small $\diamond$ ) form a continu1um extending down to Malin 1. I)warf galaxics, both dI (including the Ganssian sample, filled triangles; and the McGaugh Gaussian dwarf, inverted triangle) and dSph (•) form an extension of this relationship to faint magnitudes and low surface brightness. Tote that the relation bends down; there is an upper envelope for the surface brightness of dwarfs which is less than that observed in spirals. Also, some dSphs have a brighter surface brightness than the dIs at a given magnitude, this appears to rule out simple models of stripping (see text). The lower envelope to the spiral and dwarf sequence is a selection effect; it is difficult to find galaxies with surlace brightnesses so far below the sky surface brightness (dashed line). 
others refer to the faintest spheroidal systems as dEs or dSphs, while the compact elliptical systems such as M32 are merely ellipticals (or compact ellipticals). Other authors (e.g., Kormendy 1985) make a clearer distinction between the ellipticals and the true dwarf systems, which they refer to as dwarf spheroidals. 'l'he term dwarf elliptical, if used at all, refers to M32-type ellipticals (Kormendy \& Bender 1994).

The Binggeli (1994) terminology is rooted in the morphological similarity between the dwarf and normal early-type systems, as well as the preliminary conclusion that they formed a continuous surface brightness sequence (based on photographic photometry with poor dynamic range; Binggeli, Sandage, \& 'larenghi 1981). It. was the subscquent work of Kormendy (1985) which first showed that these two classes were distinct. It would seem to be best to not use the term dwarf elliptical at all, and stick with the clearer divisions of ellipticals (and compact ellipticals if some distinction needs to be made for the M32-1ype systems), and dwarl spheroidal. This terminology makes the division in the observational propertics of these systems clearer; dwarf spheroidals are no more "clliptical" than globular clusters are. "Their origin is more closely tied to dwarf irregular galaxies than giant ellipticals, and the morphological similarity should not be mistaken for any more than that.

In this section, then, we refer to the laint and difluse early-type galaxies as dSphs.

Although neither the dI nor dSph galaxies are apparently composed of an actual disk, they are nontheless well fit by an exponential profile (sec 83 ). I'he proximity of the dwarf scquences in Figure 95 has lead some authors to propose that stripping of faint spirals and dIs will give rise to dSphs (Kormendy 1987). However, the fact that some of the brighter dSphs have a central surface brightness which is brighter than that for similar dIs (e.g. Bothun et al. 1986), in addition to other problems 


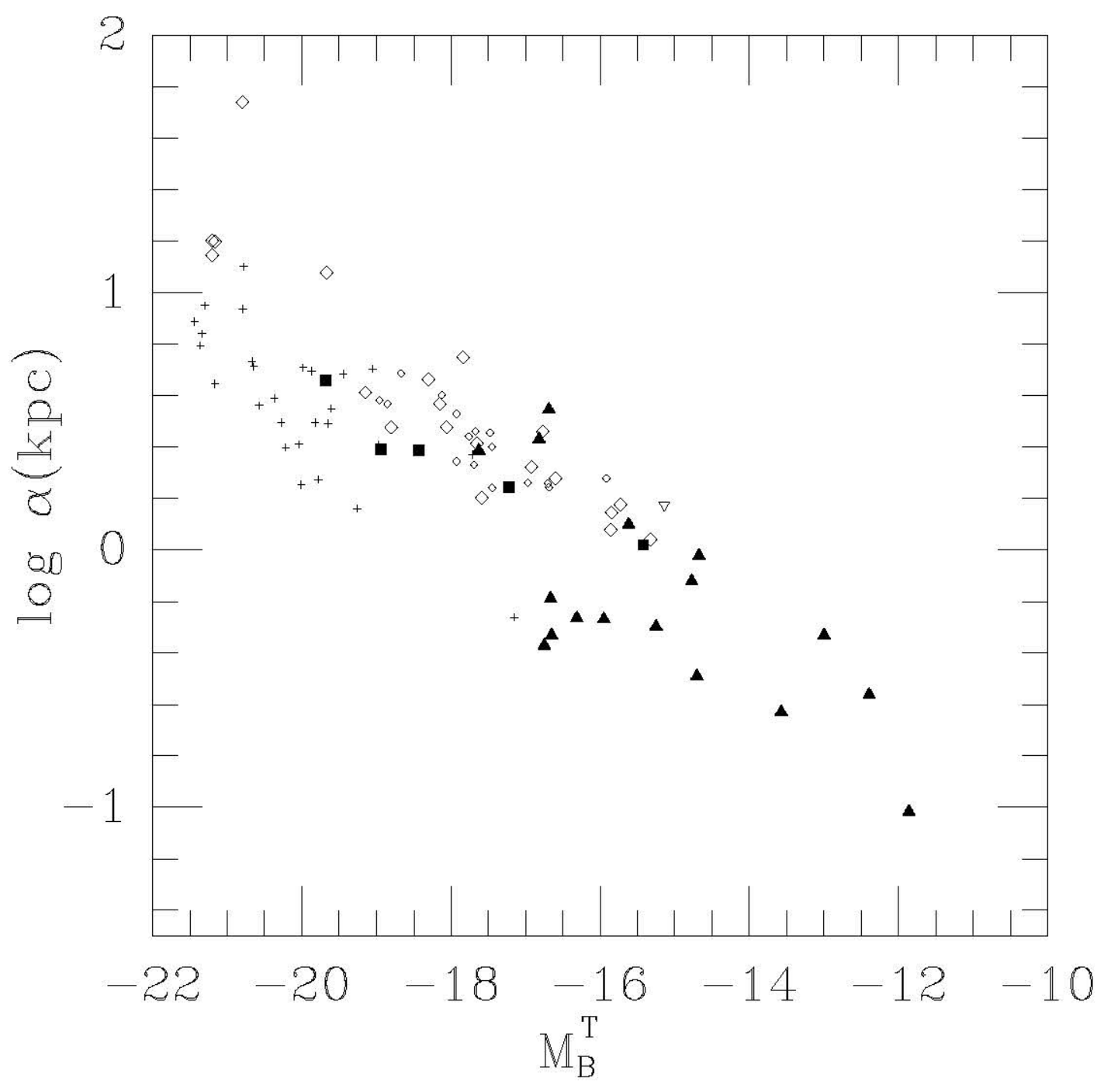

Figure 96: Disk Scale Length versus Absolute Magnitude. Symbols are as in Figure 95. There exists a tight relationship between disk scale length and magnitude. This is similar to the relation seen in Figure 93. Note that the massive LSB galaxy Malin 1 at the extreme upper lelt deviates markedly from this relationship 
including differences in the infrared colors (e.g. James, 1991), present difficulties for the stripping model.

The luminosity/surface brightness relation implies that there is a common preferred scale length for dIs and dSphs. This implies a common formation scenario for these two classes of galaxics. A common formation scenario also is implied by the fact that the Dekel-Silk relation (\$4.8) holds for both dSph and dI galaxies.

Figure 96 shows the scale length versus magnitude for the dwarf and spiral samples. Again, we see a rather tight relation for the entire sequence, excluding the Malin 1-type galaxies at the extreme upper left. These giant disk galaxies have a larger scalc Iength for a given luminosity than do the normal spirals. 


\section{Conclusions}

We have presented CCD surface photometry for $j \mathrm{l}$ dwarf and LSB galaxies in $B$ and $I$. The magnitudes obtained are used in conjunction with existing III data to investigate the Tilly Fisher relation in the regime of narrow linewidth. 'I'he dwarfs do not follow the original Tully Fisher relation because the HI linewidth arises from turbulent as well as rotational motion. If the intrinsic axial ratio of the galaxy is used to determine the degree of anisotropy in the galaxy, a modified linewidth can be determined, which is an indicator of the total mass.

The dwarls are found to follow a mass/luminosity relation which is quite distinct from that of normal spirals. While normal spirals have values of $M / L$, which increase slightly with $I$. (which results in an observed slope for the Tully Fisher relation $(\sim 7)$ that is slightly less than 10 , which is predicted for a constant $M / L$ ), the dwarfs display a definite trend of increasing $M / L$ as $L$ decreases.

The exact form of the relation was found to be well fit by the model of Dekel \& Silk (1986): $M / L \propto L^{-0.37}$. The Dekel-Silk relation arises in low mass systems with massive dark halos which undergo extensive mass loss due to supernova driven winds. The dark halo allows the galaxy to remain bound even as most (or all) of the gas is removed, drastically reducing the rate of star formation. The trend towards higher $M / L$ at lower $L$ is the result of the lower escape velocity of the less massive systems, allowing the gas to be removed more efficiently. The Dekel silk relation also appears to be present in the dwarf Spheroidal systems, indicating a common evolutionary history for these dwarfs, distinct from normal ellipticals and spirals.

The similar central surface brightness of dIs and dSphs support this conclusion. IIowever, a simple evolutionary sequence $\mathrm{dSph} \Rightarrow \mathrm{dI}$ through envirommentally- 
induced stripping seems to be ruled out by the infrared colors. Rather, the Dekel-Silk model of mass loss is envisioned as a process which occurs during the formation of the galaxy, upon the death of the very first stars formed.

Deep CCD imaging of galaxies at the extreme low-luminosity end (with independent distanecs) is needed to supplement the detailed $\mathrm{HI}$ obscrvations which already exist, in order to constrain the slope of the modified Tully-Fisher relation for dwarfs. This could then provide a useful distance indicator for gas-rich dwarfs, which would enable them to be used as test particles to examine bulk flows as well as peculiar velocitios within clusters. 


\section{References}

Aaronson, M., Bothun, G., Mould, J., Huchra, J., Schommer, R. A., \& Cornell, M. E. $1986, \Lambda p J, 302,536$

Aaronson, M., IIuchra, J., \& Mould, J. 1979, АpJ, 229, 1

Aaromson, M., IIuchra, J., Mould, J., Schecter, P. L., \& Tully, R. B. 1982, ApJ, 258, 61

Aaronson, M., \& Mould, J. 1986, Ap.J, 303, 1

Aaronson, M., \& Olszcwski, H. W. 1987, in Dark Matter in the Universe, IAU Symp 117, eds. J. Kormendy \& G. R. Knapp, (Dordrecht: Reidel), p. 153

Aparicio, A., García-Pelayo, J. M. \& Moles, M. 1988a, A\&AS, 74, 367

Aparicio, A., García-Pelayo, J. M. \& Moles, M. 1988b, A\&AS, 74, 375

Ashman, K. M. 1990, ApJ, 359, 15

Babul, A., \& Rees, M. J. 1992, MNRAS, 255, 346

Balkowski, C., Bottinelli, L., Chamaraux, P., Gouguenheim, L., \& IIeidmann, J. 1974, $\Lambda \& \Lambda, 34,43$

Bender, R., Burstein, D., \& Faber, S. M. 1992, АpJ, 399, 462

Bender, R., \& Nielo, J.-L. 1990, $1 \& \Lambda, 239,97$

Bender, R., Paquet, A., \& Nicto, J. I. 1991, A\&A, 216, 349

Binggeli, B. 1994, in FSO/OHP Workshop on Durarf Galaxies, ed. (G. Mcylan \& P. 
Prugniel (Garching: ESO), p. 13

Binggeli, B., \& Cameron, L. M. 1993, A\&AS, 98, 297

Binggeli, B., Sandage, A., \& Tarenghi, M. 1984, AJ, 89, 64

Binggeli, B., Tarenghi, M., \& Sandage, $\Lambda .1990, \Lambda \& \Lambda, 228,42$

Bimney, J. 1978, MNRAS, 183, 501

Blumenthal, G. R., Faber, S. M., Primack, J. R., \& Rees, M. J. 1984, Nature, 311, 517

Börngen, F゙., \& Karachentscva, V. F. 1985, A.I, 306, 301

Boroson, 'I'. A. 1981, Ap.JS, 16, 177

Bosma, A., \& Frecman, K. (. 1993, A.J, 106, 1391

Bothun, G. I)., Romanishin, W., Strom, S. F.. \& Strom, K. M. 1981, A.J, 89, 1300

Bothun, (.. I)., Impey, (. 1)., \& Malin, 1). F. 1991, Ap.J, 376, 104

Bothun, (x. I)., \& Mould, .J. R. 1987, Ap.J, 313, 643

Bothun, ('. I)., Mould, J. R.., Caldwcll, N., \& . Mac(xillivray, H. 'I'. 1986, A.J, 92, 1007

Bothun, G. D., Schommer, R. A., Aaronson, M., Mould, J., Huchra, J., \& Sullivan, W. 1985 , ApJS, 5i, 423

Bottinelli, L., Gouguenheim, L., Paturel, G., \& de Vaucouleurs, G. 1983, A\&A, 118, 4

Briggs, F. II. 1990, AJ, 100, 999 
Broadhurst, T. J., Ellis, R. S. \& Shanks, T. 1988, MNRAS, 235, 82i

Brosche, P. 1971 , A\&A, 13, 293

Bruzual A., G., \& Charlot, S. 1993, ApJ, 405, 538

Burstein, D. 1982, АpJ, 253, 539

Burstein, D. 1988, in Towards Inderstanding Galaxies al Large Redshifl, ed R. G. Kron \& A. Renzini (Dordrechl: Kluwer), p. 93

Burstein, D.., \& IIeiles, C. 1984, АpJS, 54, 33

Burstein, I)., \& Raychaudhury, S. 1989, Ap.J, 343, 18

Carignan, C. 1983 , Ph. 1). 'I'hesis, A.ru

Carignan, (. 1985, ApJs, 58, 107

Garignan, C., Bcaulic1, S., \& Frecman, K. C. 1990, A.J, 99, 178

Carignan, (.., I)cmers, S., \& Côtó, S. 1991, Ap.J., 381, 1.13

(arter, I). 1978, MNRAS, 182,797

(Garter, 1)., \& Sadler, F. 1990, MNRAS, 245, 12

Christian, C. A., Adams, M., Barnes, J. V., Butcher, H., Hayes, D. S., Mould, J. R., \& Siegel, M. 1985, PASP, 97, 363

Davis, L. E. 1991, private communication

Davis, L. E., Cawson, M., Davies, R. L., \& Illingworth, G. 1985, A.J, 90, 169

Dekel, $\Lambda ., \&$ Silk, J. 1986, $\Lambda$ pJ, 303, 39 
De Young, D., \& Gallagher, J. 1990, ApJL, 356, L15

De Young, D., \& Heckman. T. M. 1994, ApJ, 431, 598

Dieter, ‥ H. 1962, AJ, $6 \bar{i}, 313$

Djorgovski, S., de Carvalho, R., \& IIan, M.-S. 1988, in The Extragalaclic Distance Scale, PASPC, Vol. 4, ed S. van den Bergh \& C. J. Pritchet, (Provo: ASP), p. 329

Jjorgovski, S. \& I)avis, M. 1987, Ap.J, 313, 59

Dressler, A., Lynden Bell, 1)., Burstein, I)., Davies, R. I., Faber, S. M., Terlevich, R. J., \& Wegner, G. 1987, ApJ, 313, 42

Faber, S. M., \& Jackson, R. E. 1976, ApJ, 204,668

Fall, S. M., \& Efstathiou, G. 1980, MNRAS, 193, 189

Fall, S. M., \& Frenk, C. S. 1983, AJ, 88, 1626

Fanelli, M. N., O'Connell, R. W., \& Thuan, T. X. 1988, ApJ, 334, 665

Ferguson, H. C. 1992, in Physics of Nearby Calaxies: Nature or Nurture? ed. T. X. Thuan, C. Balkowski, \& J. T. T. VaIı (Gil-sur-Yvelte: Editions Frontières), p. 113

Ferguson, H. C., \& Binggeli, B. 1991, A\&ARev, in press

Fisher, J. R., \& 'Tully, R. B. 1981, Ap.JS, 17, 139

Frecman, K. C. 1970, Ap.J, 160, 811

Frecman, K. C. 1987, in Nearly Normal Galaxies, ed. S. Faber (Now York: Springer 
Verlag), p. 317

Franx, M., Illingworth, G., \& Heckman, T. 1989, AJ, 98, 538

Freudling, W. 1992, GALPHOT User's Manual, ESO

Gelderman, R. 1994, Ph. D. Thesis, U. Virginia

Gerola, II., Seiden, P. E., \& Schulman, L. S. 1980, ApJ, 242, 517

Ileidmann, J. 1969, AstrLett, 3, 19

IIoessel, J. G., \& Danielson, G. E. 1983, АpJ, 271, 65

IIoflman, G. L., IIelou, G., \& Salpeter, E. E. 1988, АpJ, 324, 75

IIolmberg, E. 1958, Medd. Lunds astr. Obs. 2, 136

IIubble, E. P. 1930, $\Lambda$ pJ, 71, 231

IIuchtmeier, W. K., \& Richter, O.-G. 1989 A General Calalog of III Observalions of Galaxies (Now York: Springer Verlag)

Hunter, I). A., \& Gallagher, J. S. 1985, Ap.JS, 58, 533

Hunter, 1). A., \& Gallagher, J. S. 1986, PASP, 98, 5

Hunter, D. A., Hawley, W. N., \& Gallagher, J. S. 1993, AJ, 106, 1797

Ichikawa, S., Wakamatsu, K., Okamura, S. 1986, Ap.S, 60, 475

Impey, C., Bothun, G., Malin, D. 1988, ApJ, 330, 634

Irwin, M. J., Davies, J. I., Disney, M. J., \& Phillipps, S. 1990, M`RAS, 245, 289

Izotov, Yu. I., Thuan, T. X., \& Lipovetsky, V. A. 1994, ApJ, in press 
Jacoby, G. H., Branch, D., Ciardullo, R., Davies, R. L., Harris, W. E., Pierce, M. J., Pritchet, C. J., Tonry, J. L., \& Welch, D. L. 1992, PASP, 104, 599

James, P. A. 1991, MNR AS, 250, 544

Jedrzejewski, R. I. 1987, MNR $\Lambda$ S, 226, 747

de Jong, R. S., \& van der Kruit, P. C. 1994, $\Lambda \& \Lambda S, 106,451$

Jørgensen, I., Fraix, M., \& Kjærgaard, P. 1992, $\Lambda \& \Lambda S, 95,489$

Karachentseva, V. E. 1968, Comm. Byurakan Obs. 39, 61

Karachentsev, I. 1)., \& 'Tikhonov, N. A. 1991, in ESO/OHP Workshop on Dwarf Galaxics, ed. G. Mcylan \&. P. Prugnicl (Garching: FSO), p. 109

Konnicutt, R. C. 1989, Ap.J, 311, 685

King, I. R. 1966, A.J, 71,61

Kormendy, J. 1985, ApJ. 295. 73

Kormendy, J. 1987, in Nearly Normal Calaxies, ed. S. M. Faber (New York: SpringerVerlag) p. 163

Kormendy, J., \& Bender, R. 1994, in ESO/OHP Workshop on Dwarf Calaxies, ed. G. Meylan \& P. Prugniel (Garching: ESO), p. 161

Kormendy, J., \& Djorgovski, S. 1989, $\Lambda \mathrm{R} \Lambda \& \Lambda, 27,235$

Kraan-Korteweg, R. C. 1986, $\Lambda \& \Lambda S, 66,255$

Kraan Kortewcg, R. (.., Camcron, L. M., \& 'lammann, G. A. 1988, Ap.J, 331, 620 
Landolt, A. U. 1983, AJ, 88, 439

Landolt, A. U. 1992, AJ, 104, 340

Larson, R. B., \& Tinsley, B. M. 1978, ApJ, 219, 46

Lo, K. Y., Sargent, W. L. W.. \& Young, K. 1993, AJ, 106, 507

Lu, N. Y. 1993, Ph. D. Thesis, Cormell

Lu, X. Y., IIollman, G. L., Groll, T., Roos, T., \& Lamphier, C. 1993, ApJS, 88, 383

Lu, X. Y., IIouck, J. R., Salpeter, E. E., \& Lewis, B. M. 1992, AJ, 104, 1505

Lynden-Bell, D. Faber, S. M., Burstein, D., Davies, R. L., Dressler, A., Terlevich, R., \&. Wegner, (G. 1988, Ap.J, 326, 19

Marlowe, A. 'T', Heckman, 'T'. M., Wyse, R. F'. (x., \& Shommer, R. 1994, Ap.J, in press

Massey, P. \& Jacoby, G. H. 1992, in Astronomical COD Observing and Reduction Techniques, PASPC, Vol. 23, ed. S. B. Howell (Provo: ASP), p. 240

Mateo, M. 1993, PASP, 105, 1075

Matteucci, F., \& Chiosi, C. 1983, A\&A, 123, 121

McGaugh, S. S. 1992, Ph. D. Thıesis, Univ. Michigan

McGaugh, S. S. 1994, Nature, 367, 538

McGaugh, S. S., \& Bothun, G. D. 1994, AJ, 107, 530

McNamara, B. R., Sancisi, R., IIeming, P. A.. Junor, W. 1994, AJ, 108, 844 
Meurer, G. R., Freeman, K. C., Dopita, M. A., \& Cacciari, C. 1992, AJ, 103, 60

Mihalas, D. \& Binney, J. 1981 Galactic Astronomy, 2nd edn. (San Francisco: Freeman), p. 337

Moss, C., \& de Vaucouleurs, G. 1986, P $\Lambda$ SP, 98,1282

Mould, J., IIaI, M.-S., \& Bothun, G. 1989, АpJ, 347, 112

Nieto, J.-L., Bender, R., Davoust, E., \& Prugniel, P. 1990, A\& A, 230, L17

Nilson, P. 1973, Lppsala General Catalogue of Galaxies (Uppsala Astr. Obs. Ann., v6) $[\mathrm{U}(\mathrm{G} \mathrm{C}]$

Pagel, B. F.. J., Simonson, F. A., T'rlevich, R. J., \&. Fidmunds, M. G. 1992, MNRAS, 255,325

Papaderos, P., Fricke, K. J., Thuan, T. X., \& Loose, H.-H. 1994, A\&A, 291, L13

Patterson, R. J., \& Thuan, T. X. 1992, ApJ, 400, Lij

Peimbert, M. 1986, in Star-forming Dwarf Calaxies and Related Objects, ed. D. Kunth, T. X. Thuan, and J. T. T. Van (Gil-sur-Yvette: Editions Frontières), p. 403

Pence, W. 1). 1978, U. 'Texas Publ Astr, 11

Prersic, M., \& Salucci, P. 1988, MNRAS, 231, 131

Persic, M., \& Salucci, P. 1990, Ap.J, 355, 11

Petcrson, R. C.. \&. Caldwcll, N. 1993, A.J, 105, 1111

Pieree, M. J. 1988, Ph. 1). 'l'hesis, U. Hawaii 
Pierce, M. J. 1991, in The Evolution of the Universe of Calaxies, PASPC, Vol. 10. ed. R. G. Kron (Provo: ASP), p. 48

Pierce, M. J., \& Tully, R. B. 1988, ApJ, 330, 579

Pierce, M. J., \& Tully, R. B. 1992, АpJ, 387, 47

Pierce, M. J., Welch, D. L., McClure, R. D., van den Bergh, S., Racine, R., \& Stetson, P. B. 1994, Nature, 371, 385

Puche, 1)., Wostpfahl, 1)., Brinks, E.., \& Roy, J. R. 1992, A.J, 103, 1811

Pustil'nik, S. A., Ugryumov, A. V., lipovetsky, V. A., T'huan, 'I'. X., \&. Guscva, N. G. $1994 a, \mathrm{ApJ}$, in press

Pustil'nik, S. A., Ugryumov, A. V., Lipovetsky, V. A., Thuan, T. X., \& Salzer, J. J. 1994b, in ESO/OHP Workshop on Dwarf Galaxies, ed. G. Meylan \& P. Prugniel (Garching: ESO), p. 133

Rees, M. J. 1985, MNR $\Lambda$ S, 213, 75P

Reynolds, J. II. 1913, MNRAS, 74, 132

Roberts, M. S. $1962 a$, AJ, 67, 431

Roberts, M. S. $1962 b$, A.J, 67,137

Roberts, M. S. 1978 , A.J, 83, 1026

Rogstad, I). H., \&. Shostak, G. S. 1972, ApJ, 176, 315

Romanishin, W., Strom, K., Strom, S. 1983, ApJS, 53, 105

Rubin, V. C., Burstcin, I)., Ford, Jr., W. K., \& Thonnard, I. 1985, Ap.J, 289, 91 
Salzer. J. J. 1989, ApJ, 347, 152

Salzer. J. J., Hanson, M. M., \& Gavazzi, G. 1990, ApJ, 353, 39

Salzer, J. J., \& Rosenberg, J. L. 1994, in ESO/OHP Workshop on Dwarf Galaxies, ed. G. Meylan \& P. Prugniel (Garching: ESO), p. 129

Sancisi, R., \& van Albada, T. S. 1987, in Dark Maller in the Universe, IAL Symp 117, ods. J. Kormendy \& (4. R. Knapp (I)ordrecht: Reidel), p. 67

Sandage, A. 1985 , private communication

Sandage, A. 1986, in Star forming Dwarf Galaxics and Related Objects, ed. 1). Kunth, T. X. Thuan, and J. T. T. Van (Gif-sur-Yvette: Editions Frontières), p. 31

Sandage, A. 1994a, ApJ, 430, 13

Sandage, A. 1994b, $\mathrm{ppJ}, 430,29$

Sandage, A., \& Binggeli, B. 1984, AJ, 89, 919

Sandage, A., Freeman, K. C., \& Stokes, N. R. 1970, АpJ, 160, 831

Sandage, A., \& IIoflman, G. L. 1991, ApJL, 379, L45

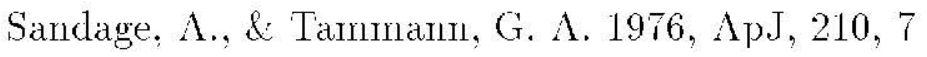

Sargenl, W. L. W., Sancisi, R., \& Lo, K. Y. 1983, ApJ, 265, 711

Schecter, P. L. 1980, AJ, 85, 801

Scheffler, H., \& Vilsässcr, H. 1988, Physics of the Galaxy and Interstellar Matter, (Berlin: Springer Verlag), p. 156 
Schild, R. E. 1983, PASP, 95, 1021

Schmidt, K.-H., \& Boller, T. 1992, AN, 313, 189

Schmidt, R., Richter, G. M., \& Karachentseva, V. E. 1985, AN, 306, 257

Schneider, S. E., \& Thuan, T. X. 1989, private communication

Schneider, S. E., Thuan, T. X., Magri, C., \& Wadiak, J. E. 1990, ApJS, 72, 245

Schneider, S. E., Thuan, T. X., Mangum, J. G., \& Miller, J. 1992, АpJS, 81, 5

Schweizer, F. 1992, in Physics of Nearby Galanies: Nalure or Nurlure? ed. T. X. 'l'huan, C. Balkowski, \& J. I'. T. Van (Gif-sur-Yvette: Fiditions Frontic̀res), p. 28.3

Silk, J., Wyse, R. F. G., \& Shiclds, G. A. 1987, Ap.Jl, 322, I.59

Smith, H. A., \& 'linsley, B. M. 1976, PASP, 88,370

Sprayberry, D., Impey, C. D., Irwin, M., McMahon, R. G., \& Bothun, G. D. 1993, ApJ, 417,114

Staveley-Smith, L., Davies, R. D., \& Kinman, T. D. 1992, 258, 334

Stetson. P. B. 1987, PASP, 99, 101

Strobel, N. V., Ilodge, P., \& Kennicutt, R. C. 1991, АpJ, 383, 148

Tammann, G. A. 1980, in ESO Reporl of Workshop on Dwarf Galaries, ed. M. Tarenghi and K. Khar, p. 3

Taylor, (.. I., Brinks, F., \& Skillman, Fi. I). 1993, A.J, 105, 128 
Thuan, T. X. 1986, in Star-forming Dwarf Galaxies and Related Objects, ed. D. Kunth, T. X. Thuan, \& J. T. T. Van (Gif-sur-Yvette: Editions Frontières), p. 129

Thuan, T. X. 1991, in Massive Slars in Slarbursls, eds C. Leitherer, N. R. Walborm, I'. M. Heckman, \&. (.. A. Norman (Cambridge: Cambridge Liniv. Press), 183

Thuan, 'I: X., Alimi, J. M., Gott, J. R., \& Schneider, S. F. 1991, Ap.J, 370,25

'Thuan, T. X., Izotov, Y1. I., lipovetsky, V. A., \& Pustilnik, S. A. 1994, in FSO/OHP Workshop on Dwarf Galaxies, ed. G. Meylan \& P. Prugniel (Garching: ESO), p. 421

Thuan, T. X., Palterson, R. J., Condon, J. J., \& Mitchell, K. J. 1992, AJ, 104, 1331

Tikhonov, N. A., \& Karachentsev, I. D. 1993, $\Lambda \& \Lambda, 275,39$

Toomre, $\Lambda .1964, \Lambda p J, 139,1217$

Tremaine, S. \& Gunn, J. E. 1978, Phys Rev Letlers, 42, 407

Tully, R. B. 1988 Nearby Galaxies Calalog (Cambridge: Cambridge Univ. Press) $[\therefore B(\bar{T}]$

Trully, R. B., Bottinclli, L.., Fisher, J. R., Gouguenheim, I., Sancisi, R., van Woerden, H. 1978, A\&A, 63,37

Tully, R. B., \& Fouqué, P. 1985, ApJS, 58, 67

Tully, R. B., \& Fisher, J. R. 1977, A\& A, 54,661

Tully, R. B., \& Fisher, J. R. 1987 Nearby Galaxies Atlas (Cambridge: Cambridge Univ. Press) 
Tyson, J. A. 1988, AJ, 96, 1

Vader, J. P. 1986, ApJ, 305, 669

Vader, J. P. 1987, ApJ, 317, 128

van de Rydi, F., Demers, S., \& Kunkel, W. E. 1991, AJ, 102, 130

van den Bergh, S. 1981, АJ, 86, 1464

vaIı deIı Bergh, S. 1994, АJ, 107, 1328

van der IIulst, J. M., Skillmaı, E. D., Smith, T. R., Bothum, G. D., McGaugh, S. S., \& de Blok, W. J. (7. 1993, A.J, 106, 518

van der Kruit, P. C., \& Shostak, G. S. 1982, A\&.A, 105, 351

de Vaucouleurs, G. 1918, AnnAp, 11, 247

do Vaucoulcurs, G. 1959, in Handbuch der Physik, y 53, ed. S. Flügge (Berlin: Springer-Verlag), p. 275

de Vaucouleurs, G., \& Moss, C. 1983, ApJ, 271, 123

de Vaucouleurs, G., de Vaucouleurs, A., \& Buta, R. 1983, A.J, 88, 764

de Vaucouleurs, G., de Vaucouleurs, A., Corwin, H. G., Jr., Buta, R. J., Paturel, G., \& Fouqué, P. 1991. The Third Reference Calalogue of Brighl Galaxies (New York: Springer-Verlag) [RC3]

Veminik, J., \& Richıler, G. M. 1994, АN, 315, 245

Weinberg, I). H., Szomoru, A., Guhathakurta, P., \& van Gorkom, J. H. 1991, Ap.J,, $372,1.13$ 
Westpfahl, D., \& Puche, D. 1993. A.J, submitted

Wirth, A., \& Gallagher, J. S. 1984, ApJ, 282, 85

Wyse, R. F. G. 1993, in The Environment and Evolution of Galaxies, ed. J. M. Shull \& II. A. Thromson, Jr. (Dordrechl: Kluwer) p. 305

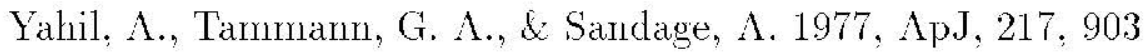

Zaritsky, D. \& White, S. D. M. 1994, in ESO/OIIP Workshop on Dwarf Galaxies, cd. (G. Mcylan \& P. Prugnicl (Garching: FSO), p. 355

\%wicky, F'. 1933, Hclv. Phys. Acta, 6, 110

7wicky, F., Herzog, F., Will, P., Karpowicz, M., \& Kowal, C. 1960 1968, Catalog of Calaxies and Clusters of Galaxies (Pasadena: Caltech) 